\title{
Investigating the Calcium Signaling at Ribbon Synapses
}

\author{
PhD Thesis \\ (Cumulative thesis) \\ in partial fulfilment of the requirements \\ for the degree "Doctor of Philosophy $(\mathrm{PhD}) / \mathrm{Dr}$. rer. nat." \\ in the Neuroscience Program \\ at the Georg August University Göttingen, \\ Faculty of Biology
}

submitted by

Thomas Frank

born in

Starnberg

Göttingen, March 17, 2010 
Declaration

Herewith I declare that this thesis has been written independently and with no other sources and aids than quoted.

Thomas Frank

Göttingen, March 17, 2010 


\section{List of Contents}

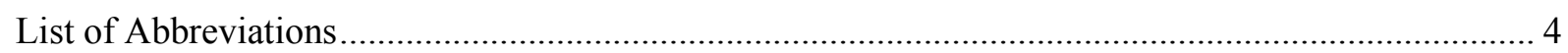

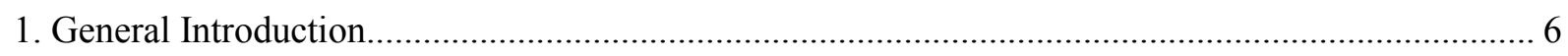

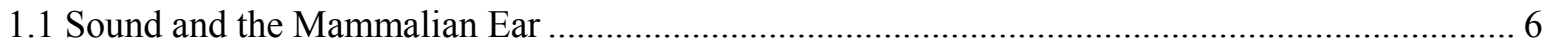

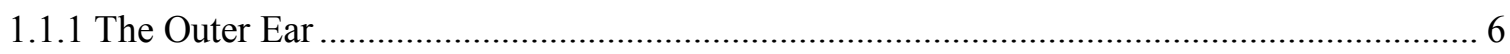

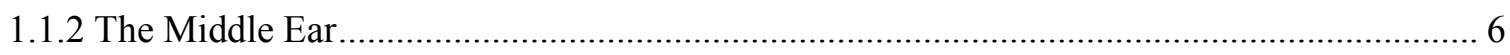

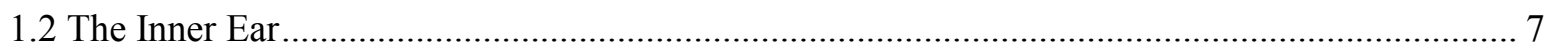

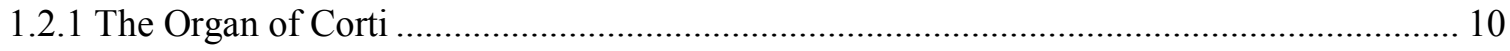

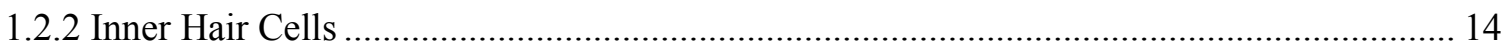

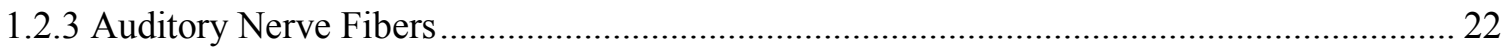

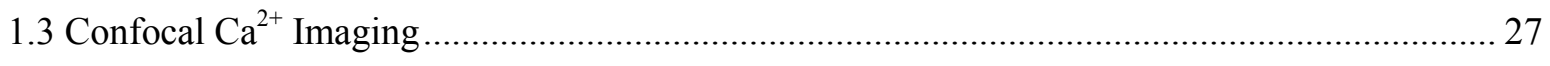

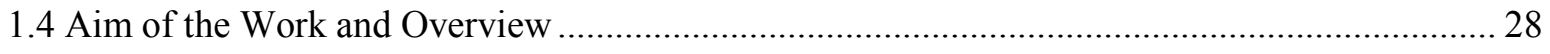

2. Mechanisms Contributing to Synaptic $\mathrm{Ca}^{2+}$ Signals and Their Heterogeneity in Hair Cells............. 30

3. Tuning of Synapse Number, Structure and Function in the Cochlea .............................................. 46

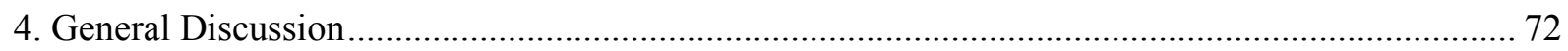

4.1 Comparison to $\mathrm{Ca}^{2+}$ Microdomains in Other Hair Cell Types .................................................... 72

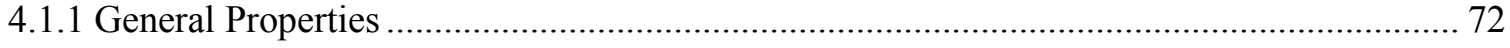

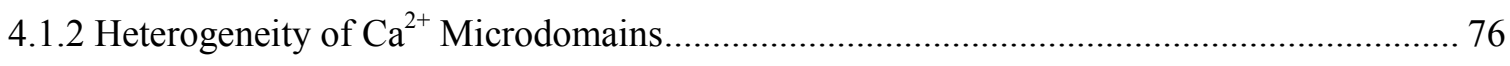

4.2 Potential Artefacts in $\mathrm{Ca}^{2+}$ Microdomain Amplitude Estimation............................................. 78

4.3 Mechanisms Contributing to $\mathrm{Ca}^{2+}$ Microdomain Heterogeneity ............................................... 82

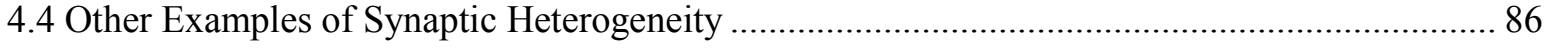

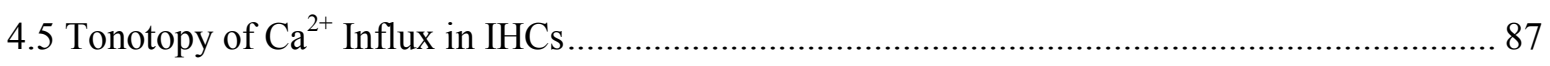

4.6 Relevance of $\mathrm{Ca}^{2+}$ Microdomain Heterogeneity for Sound Coding .......................................... 89

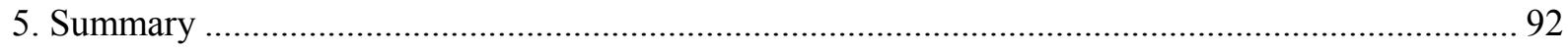

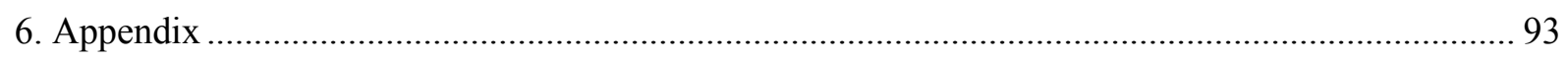

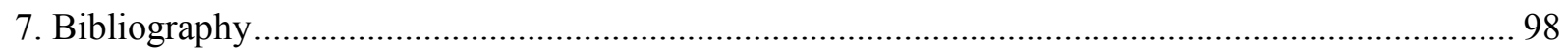

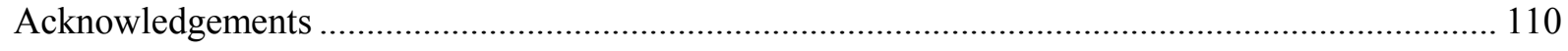

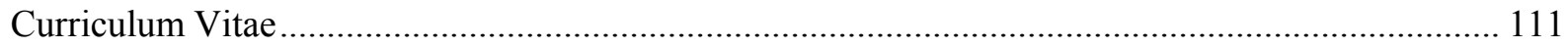

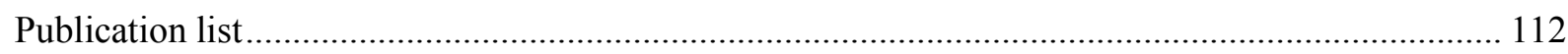




\section{List of Abbreviations}

$\mathrm{ACh}$

AMPA

ANF

ATP

BAPTA

$\left[\mathrm{Ca}^{2+}\right]_{\mathrm{i}}$

$\left[\mathrm{Ca}^{2+}\right]_{\mathrm{e}}$

CF

CICR

CLSM

CV

DA

$\Delta \mathbf{F}$

DHP

DR

EGTA

EPSC

$\mathbf{F}_{\mathbf{0}}$

GABA

GTP

$\mathbf{I}_{\mathbf{C a}}$

$\mathbf{i}_{\text {Ca }}$

IHC

$\mathbf{K}_{\mathbf{D}}$

LOC

LSO

MET channel

MOC

MSO

MVR

$\mathbf{N}$

NA acetylcholine

$\alpha$-amino-3-hydroxy-5-methyl-4-isoxazolepropionic acid

auditory nerve fiber

adenosine 5'-triphosphate

1,2-bis(2-aminophenoxy)ethane- $N, N, N 9, N 9$-tetraacetate

intracellular calcium concentration

extracellular calcium concentration

characteristic frequency

calcium-induced calcium release

confocal laser-scanning microscopy

coefficient of variation

dopamine

relative change in fluorescence

dihydropyridine

dynamic range

ethylene glycol-bis-(2-aminoethyl)-N,N,N',N'-tetraacetic acid

excitatory postsynaptic current

pre-stimulus fluorescence

$\gamma$-amino-butyric acid

guanosine 5'-triphosphate

whole-cell calcium current

unitary calcium current

inner hair cell

dissociation constant

lateral olivocochlear bundle

lateral portion of superior olivary complex

mechanoelectrical transducer channel

medial olivocochlear bundle

medial portion of superior olivary complex

multivesicular release

total number of a certain ion channel population per cell

numerical aperture 


\begin{tabular}{ll} 
NMDA & N-methyl-D-aspartate \\
OHC & outer hair cell \\
PMT & photomultiplier tube \\
popen $_{\text {PSF }}$ & open probability \\
PSTH & point-spread function \\
R & post-stimulus time histogram \\
sd (SD) & series resistance \\
s.e.m. (SEM) & standard deviation \\
SGN & standard error of the mean \\
SOC & spiral ganglion neuron \\
SR & superior olivary complex \\
STED & spontaneous rate \\
SV & stimulated emission depletion \\
TEA & synaptic vesicle \\
VGCCs & tetraethylammonium \\
\hline
\end{tabular}




\section{General Introduction}

\subsection{Sound and the Mammalian Ear}

Sound, in physical terms, refers to a periodic, elastic compression and rarefaction of the transmitting medium, for example air. Sound hence propagates in the form of longitudinal waves (but spherically from the source) and can be described by waveform, amplitude, phase, and frequency. Over the vast range of naturally occurring sound pressure levels, these pressure changes are sensed by the auditory system. Different mammalian species differ in their hearing range, i.e. the range of frequencies that elicit a behavioral or physiological response in a given subject/animal. Whereas humans have a hearing range of approximately $20-20,000 \mathrm{~Hz}$ (with considerable inter-individual and age-related differences; Brugge and Howard 2002), the hearing range of mice covers frequencies from around 1,000 to about 100,000 Hz (Ashmore 2008). These differences have important implications for the way certain information about frequency and localization of sound is encoded and subsequently processed by the nervous system of the respective animal. The mammalian ear consists of three parts, the outer, the middle, and the inner ear, which will be topic of the following paragraphs. Unless stated otherwise, the following sections describe the morphology and general function of the human ear, while most of the physiological background necessarily recapitulates experiments performed in rodents or cats, and partly also other vertebrates. For a comprehensive description of the entire auditory system see Møller (2006).

\subsubsection{The Outer Ear}

The outer or external ear comprises the pinna (auricle) and the external ear canal (external auditory meatus; Fig. 1). One task of the pinna is to gather sound and to focus it into the external ear canal, somewhat similar to the function a satellite dish. Moreover, the outer ear plays a role in physical protection of the ear, in sound-localization, and in frequency-selective amplification by means of a passive resonance mechanism (in the range of $\approx 1.5-7 \mathrm{kHz}$; Brugge and Howard 2002).

\subsubsection{The Middle Ear}

The tympanic membrane (or eardrum) is located at the end of the external ear canal. It vibrates in response to sound and anatomically separates the outer and the middle ear. The middle ear, moreover, comprises the air-filled middle-ear cavity and the three auditory ossicles: malleus (hammer), incus (anvil), and stapes (stirrup) (Fig. 1). The basic function of the middle 
ear is impedance matching, as for sound transduction air-borne vibrations have to be converted to vibrations in a fluid-filled space (inner ear). Without this adjustment, the largest fraction of acoustical energy would be reflected at the oval window. The impedance matching is accomplished by two mechanisms: first, the leverage created by the three auditory ossicles and, second, the difference in area between the tympanic membrane and the oval window to which the footplate of the stapes is attached to. The latter mechanism results in a pressure difference that is proportional to the ratio of the two areas (approximately 20-fold; Brugge and Howard 2002). Furthermore, the middle-air-cavity is connected to the pharynx via the Eustachian tube; this connection allows the air pressure on both sides of the tympanic membrane to be equilibrated in order to protect the ear from potential damage upon changes of external air pressure. Last, the middle ear contains two muscles that allow for some degree of regulation of the mechanical transmission within the middle ear - by stiffening/loosening of the ossicular chain or by altering the tension of the tympanic membrane.

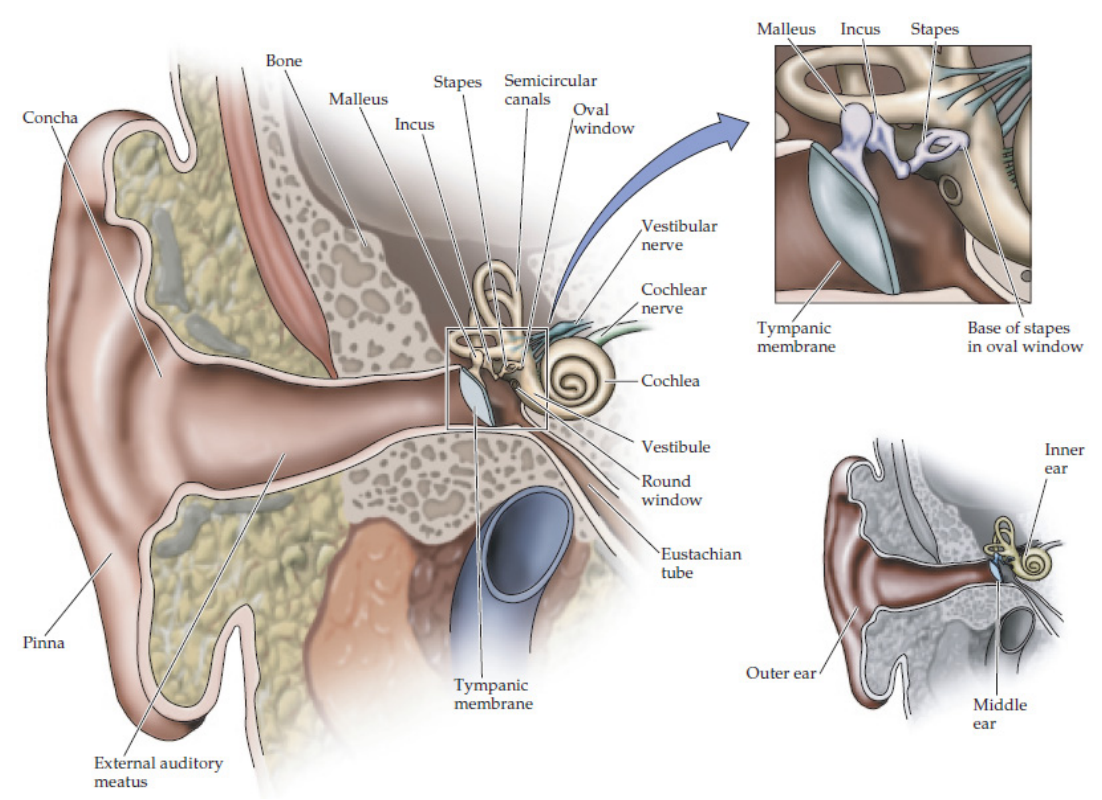

Figure 1 | The human ear. Schematic representation of the main components of the outer, middle, and inner ear. The upper right panel shows a cutout of the auditory ossicles in the middle ear, and their position relative to the eardrum and the inner ear. The lower right panel outlines the three main divisions of the ear (taken from: Purves et al. 2004).

\subsection{The Inner Ear}

The inner ear harbors the sensory organs of both the auditory (hearing) and the vestibular sense (balance). Whereas the auditory organ is located in the cochlea, the vestibular epithelia 
- utricle, saccule, and the ampullae in the semicircular canals - are found in the vestibular labyrinth, which is, however, continuous with the cochlea. Next to their similar location, and developmental origin, the two different sensory organs, in hair cells, also use the same type of receptor cells (see below).

The main function of the cochlea is the encoding of the spectral and temporal information in the sound impinging on the eardrum. To this end, the sound is divided up into its distinct spectral components and the time-dependent intensities of those components are encoded into spike rates in the auditory nerve, which is also organized by sound frequency. The cochlea is a small, coiled structure surrounded by bone (Figs. 1,2). In humans, it has slightly more than 2.5 turns (Møller 2006), but this number differs from species to species. It has two regions at its basal end from which the bone is absent: (i) the oval window and (ii) the round window. Onto the oval window, the stapes is attached, whereas the round window is solely covered by an elastic diaphragm.

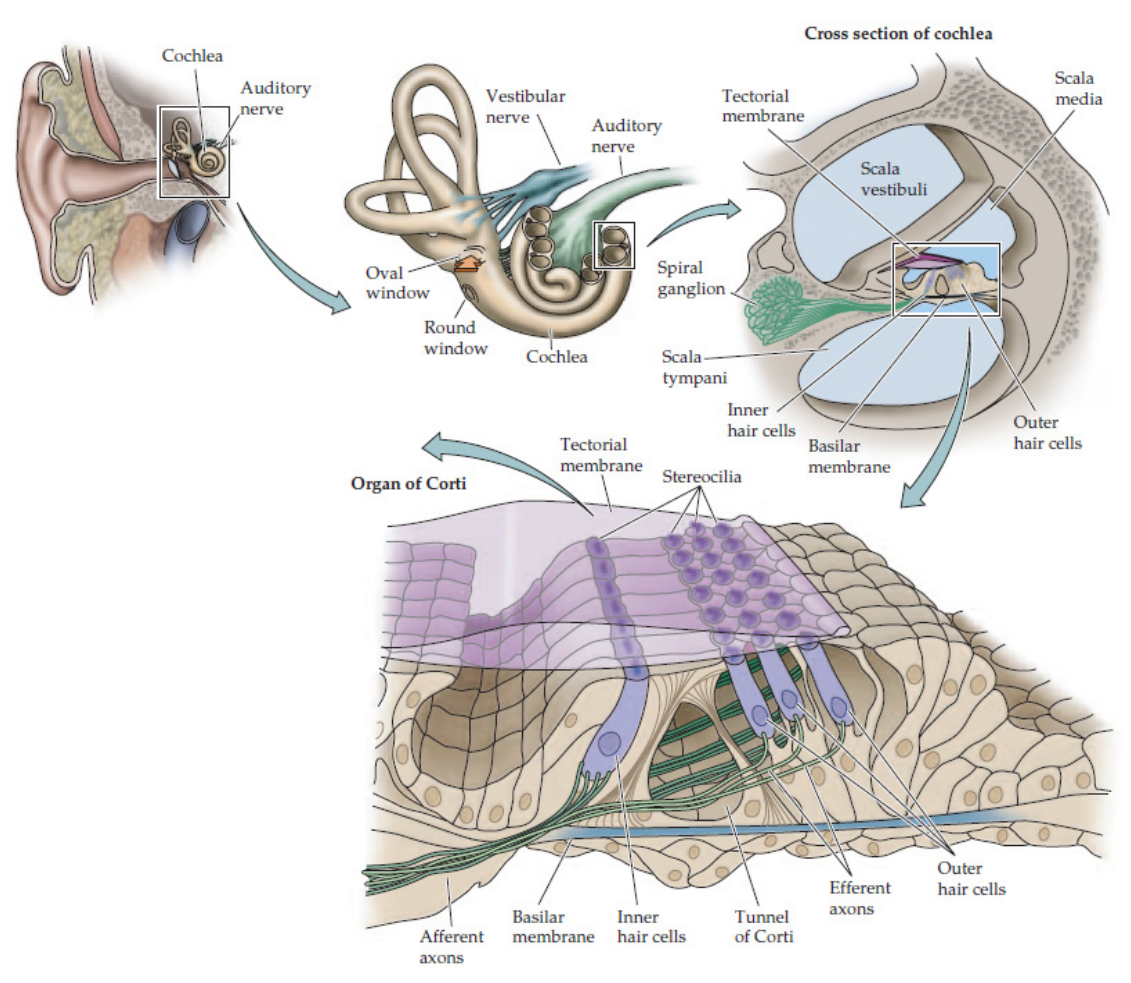

Figure 2 The cochlea and the organ of Corti. The cochlea viewed face-on at its position within the ear (upper left) and in cross section (subsequent panels), showing the positions of the oval and the round window, the originating auditory nerve, and, at increasing detail, the location and fine-structure of the organ of Corti, the sensory epithelium (schematic taken from: Purves et al. 2004).

Within the cochlea - along its midline and for almost its entire length - runs the cochlear partition. It consists of two membranes (Reissner's membrane and basilar membrane) and thus 
separates three fluid-filled compartments from each other. The scala vestibuli (Fig. 2, upper right panel) contacts the oval window and - along the cochlear partition - is bordered by Reissner's membrane. At the helicotrema, the very apical part of the cochlea, it is continuous with the scala tympani. The scala tympani (Fig. 2, upper right panel) is bordered by the basilar membrane and is in contact with the round window. Although there are slight differences in the ionic composition between these two fluids (see Table 1), they generally are very similar to the extracellular media found in other tissues, namely having high concentrations of sodium $\left(\left[\mathrm{Na}^{+}\right]_{\mathrm{e}}\right)$ and chloride ions $\left(\left[\mathrm{Cl}^{-}\right]_{\mathrm{e}}\right)$. The third fluid-filled compartment, the scala media (cochlear duct), runs within the cochlear partition (Fig. 2, upper right panel) and harbors the organ of Corti and the tectorial membrane (Fig. 2). The tectorial membrane is composed of extracellular matrix material and plays an important role in sound transduction (Legan et al. 2000). The scala media is delimited dorsally by Reissner's membrane and ventrally by the basilar membrane. The basilar membrane varies in width and stiffness along the length of the cochlea: While it is narrow $(\approx 150 \mu \mathrm{m})$ and rigid at the base (close to oval and round window), it is wider $(\approx 450 \mu \mathrm{m})$ and more limp towards the apex of the cochlea (Møller 2006). On top of the basilar membrane resides the organ of Corti - the sensory epithelium (see below).

Table 1 | Inner ear fluid composition. Table modified from (Wangemann and Schacht 1996). Values report activities for $\mathrm{Ca}^{2+}$ and $\mathrm{pH}$ but concentrations for all other components and represent averages from data obtained in guinea pigs and rodents.

\begin{tabular}{|l|c|c|c|c|}
\hline \multicolumn{1}{|c|}{ Component } & $\begin{array}{c}\text { Cochlear } \\
\text { endolymph }\end{array}$ & $\begin{array}{c}\text { Perilymph (sca- } \\
\text { la vestibuli) }\end{array}$ & $\begin{array}{c}\text { Perilymph (sca- } \\
\text { la tympani) }\end{array}$ & $\begin{array}{c}\text { Cerebrospinal } \\
\text { fluid }\end{array}$ \\
\hline $\mathrm{Na}^{+}(\mathrm{mM})$ & 1.3 & 141 & 148 & 149 \\
\hline $\mathrm{K}^{+}(\mathrm{mM})$ & 157 & 6.0 & 4.2 & 3.1 \\
\hline $\mathrm{Ca}^{2+}(\mathrm{mM})$ & 0.023 & 0.6 & 1.3 & 1.2 \\
\hline $\mathrm{Cl}^{-}(\mathrm{mM})$ & 132 & 121 & 119 & 129 \\
\hline $\mathrm{HCO}^{3-}(\mathrm{mM})$ & 31 & 18 & 21 & 19 \\
\hline $\left.\mathrm{Glucose}^{\prime m M}\right)$ & 0.6 & 3.8 & 3.6 & 4.8 \\
\hline Urea $(\mathrm{mM})$ & 4.9 & 5.2 & 5.0 & 5.2 \\
\hline Protein $(\mathrm{mg} / 100 \mathrm{ml})$ & 38 & 242 & 178 & 24 \\
\hline $\mathrm{pH}$ & 7.4 & 7.3 & 7.3 & 7.3 \\
\hline
\end{tabular}

The scala media is unusual with respect to its high potassium ion $\left(\left[\mathrm{K}^{+}\right]_{\mathrm{e}}\right)$ and low sodium ion $\left(\left[\mathrm{Na}^{+}\right]_{\mathrm{e}}\right.$ ) concentration (Table 1). The high $\mathrm{K}^{+}$concentration is a result of active ion transport processes carried out by several different cell types in the stria vascularis, a multi-layered epithelium situated at the lateral wall of the cochlear duct (Wangemann and Schacht 1996). The endolymph in the scala media has a potential of approximately $+80 \mathrm{mV}$ relative to the peri- 
lymph-filled compartments; this potential is called the endocochlear potential and, again, depends on the ion transport activity of the stria vascularis (Nin et al. 2008). The energy for the sound transduction process is stored in the resulting large electric gradient between the endolymphatic space $(\approx+80 \mathrm{mV}$, endocochlear potential) and the membrane potential of hair cells $(\approx-45 \mathrm{mV})$.

The energy of the sound wave is transmitted into the fluid-filled compartments of the cochlea by the auditory ossicles of the middle ear and causes vibrations of the basilar membrane and the overlying sensory hair cells. Due to the virtually incompressible nature of fluids, the main effect of an oval window movement is a deflection of the elastic cochlear partition alongside the propagating sound wave within the cochlea; both the sound wave and its accompanying deflection hence travel from the cochlea's base to its apex. In consequence, the traveling wave also causes pressure changes in the scala tympani. At the basal end of the scaly tympani, the flexible round window membrane (which vibrates at opposite phase to the oval window membrane) allows for significant fluid movement within the cochlea and thus for an efficient transfer of acoustical energy across the fluid compartments of the cochlea. As a result of the non-uniform mechanical properties of the basilar membrane along the cochlea (see above), its resonance frequency also varies from base to apex. Hence, a given part of the basilar membrane will oscillate strongest for a distinct sound frequency and the acoustic energy transferred between the different fluid compartments will be largest around this location (for this frequency band). On top of this passive motion of the basilar membrane, the outer hair cells interact actively as a positive feedback regulator with the deflection of the basilar membrane (but see: Reichenbach and Hudspeth 2010). It is due to the resonance gradient of the basilar membrane that it is said to be tonotopically organized. Physical separation of sound energy in its spectral components is thus achieved by the passive resonance gradient, provided by the basilar membrane, and its amplification by outer hair cells. The tonotopic organization is picked up by the very focal innervation of auditory nerve fibers (ANFs; see below).

\subsubsection{The Organ of Corti}

The epithelium that subsequently converts the intensities within the physically decomposed frequency bands into electrical signals - the organ of Corti - consists of several different cell types. Among them are two classes of hair cells: inner hair cells (IHCs), the principal sensory cells, and outer hair cells (OHCs), which constitute the substrate of the active process of 
sound amplification in the cochlea ('cochlear amplifier', see below; for review: Fettiplace and Hackney 2006). Additionally, there are several types of supporting cells, amongst them: border cells, pillar cells, phalyngeal cells, Hensen's cells, Claudius's cells, and Deiter's cells. They are mainly thought to play a role in ion homeostasis and mechanical support and stabilization, without being directly involved in the process of sound transduction.

While IHCs and OHCs differ from each other in a variety of morphological and physiological properties (Fettiplace and Hackney 2006), their basic mode of excitation is very similar. Each hair cell, polarized cells of epithelial origin, carries a so called hair bundle at its apical pole. The hair bundle is formed by a regular array of about 50-150 small membrane protrusions termed stereocilia. The, microvilli-related, stereocilia have a core of actin filaments and are inter-connected by different extracellular linkers of mostly proteinaceous nature (Müller 2008). Depending on the type of hair cell, the hair bundles are in functional and/or direct contact with the tectorial membrane: by now, it is generally agreed that at least the longest of the three rows of OHC stereocilia is attached to the tectorial membrane (Slepecky 1996). Upon vibration of the basilar membrane, the relative movement of the organ of Corti with respect to the tectorial membrane causes a shearing motion of the hair bundle and consequently the deflection of its stereocilia. Extracellular links between individual stereocilia, hydrodynamic coupling, or mechanical constraints imposed by the stereocilias' insertion at the apical hair cell surface may explain the observation that the hair bundle moves as an entity (Kozlov et al. 2007). The stereocilia harbor the mechanically gated mechanoelectrical transducer (MET) channels, which convert the mechanical stimuli into electrical signals in hair cells. The MET channel is a large conductance cation channel of unknown molecular identity (Fettiplace 2009). In rats, it has recently been shown that MET channels are present only in the two smallest rows of stereocilia (Beurg et al. 2009). Moreover, this study suggests that there are on average only two MET channels per stereocilium. Shearing of the hair bundle towards the largest stereocilia increases the open probability $\left(\mathrm{p}_{\text {open }}\right)$ of the MET channels, whereas deflec-

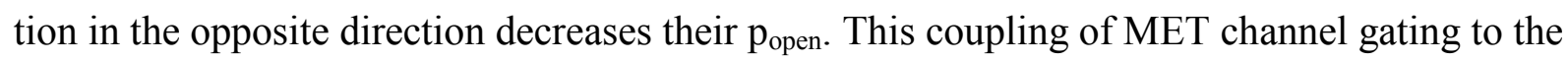
movement of stereocilia is thought to be accomplished by tip links, a certain type of extracellular, proteinaceous linker that physically connect the channel 'gate' with its neighboring, larger stereocilium (Kazmierczak et al. 2007, Müller 2008). As stereocilia are surrounded by endolymph, the transduction current through MET channels is largely carried by $\mathrm{K}^{+}$ions and to a smaller degree by $\mathrm{Ca}^{2+}$ ions (table 1; Lumpkin et al. 1997). 
Although their hair bundles operate in a similar fashion, OHCs and IHCs serve fundamentally different roles in cochlear function. The cylindrical-shaped OHCs constitute the cellular basis of the cochlear amplifier (Hudspeth 2008, Ashmore 2008). By this electromechanical positive feedback mechanism, which seems to involve both active motility of the OHC hair bundle as well as electromotility of OHCs (Reichenbach \& Hudspeth, 2010, Ashmore et al., 2010), the electrical change in OHCs generates a movement of the cell's bundle that increases the amplitude of its deflection ('reverse transduction'). This non-linearity locally boosts mechanotransduction in neighboring IHCs, whose stereocilia are probably deflected by radial flux, and thus increases cochlear sensitivity (by approximately $50 \mathrm{~dB}$ ) and sharpens cochlear frequencytuning, especially at its base (Robles and Ruggero 2001).

The organ of Corti is innervated by three types of nerve fibers: (i) afferent auditory nerve fibers, (ii) efferent auditory fibers (running in the olivocochlear bundle), and (iii) autonomic nerve fibers (Eybalin 1993). Together with the vestibular nerve that innervates the vestibular labyrinth, the auditory nerve forms the VIII ${ }^{\text {th }}$ cranial nerve (vestibulo-cochlear nerve).
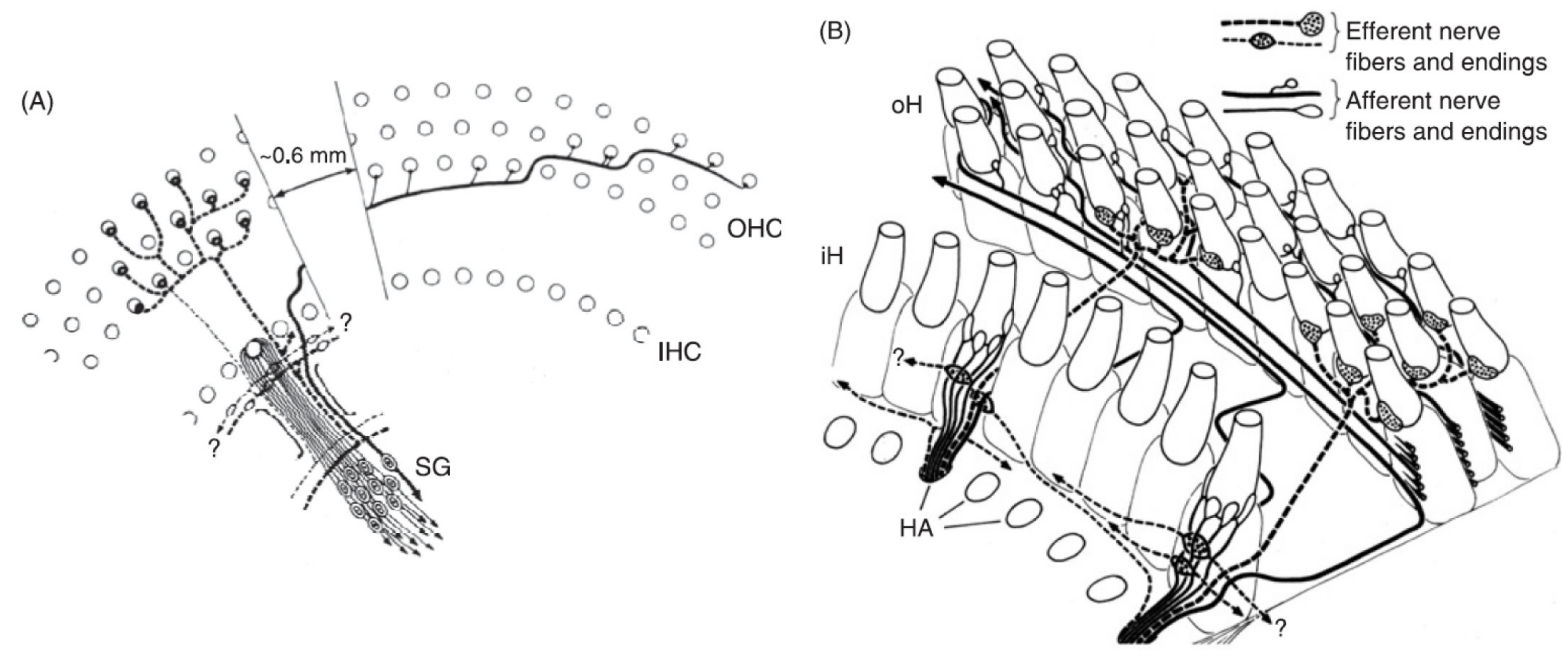

Figure 3 | Innervation pattern of hair cells by auditory nerve fibers (taken from: Møller 2006). $\mathrm{IHC} / \mathrm{iH}$ : inner hair cell, $\mathrm{OHC} / \mathrm{oH}$ : outer hair cell, SG: spiral ganglion, HA: habenulae openings.

Auditory nerve fibers (ANFs) are bipolar cells. In humans, the auditory nerve consists of approximately 30,000 fibers (Møller 2006). The cell bodies of ANFs are located in the spiral ganglion, situated in the modiolar region of the cochlea (Fig. 2, upper right panel, Fig. 3). The peripheral portion of the ANF terminates on hair cells, whereas the central portion projects to the cochlear nucleus in the lower brainstem, the first relay nucleus in the ascending auditory system. The afferent ANFs can be further subdivided into two - non-overlapping - groups. The vast majority of ANFs (90-95\%) is myelinated and contacts IHCs (Fig. 3). They are 
called type I spiral ganglion neurons giving rise to inner radial fibers in the cochlea. There is a strong degree of divergence between IHCs $(\approx 3,500$ per cochlea; Møller, 2006) and ANFs $(\approx 30,000$ per nerve; see above). In most mammalian species studied so far, the vast majority of type I fibers have a single unbranched peripheral process that contacts one IHC by means of a single synaptic contact (Slepecky 1996). However, based on post-mortem studies, a much more common branching of the peripheral process of type I ANFs, with a single ANF innervating up to 3 neighboring IHCs, was reported for humans (Nadol 1983). In both setups of innervation, nonetheless, a given ANF receives its input from an extremely defined region in the cochlea, and, thus, a narrow band of frequencies. In consequence, the frequency selectivity of a given ANF is to a large degree determined by the location of the innervated IHC(s) along the tonotopic axis ('place code'; Liberman 1982a). The innervation density seems to vary along the cochlea, for example up to three-fold in cat, with a peak between the apical and the basal region of the cochlea, in the region of the highest auditory sensitivity (Liberman et al. 1990, Francis et al. 2004; see also: chapter 3, Fig. 1).

Outer hair cells, in contrast, are innervated by unmyelinated type II ANFs (spiral fibers) that constitute the remainder of nerve fibers running within the auditory nerve (about 5\%). Their innervation pattern is very different from the one of type I fibers (Fig. 3): one type II fiber branches extensively (sometimes extending several $100 \mu \mathrm{m}$ along the rows of OHCs) and innervates many different OHCs. A strong base-to-apex increase in type II ANF innervation of OHCs has been reported in cats (Liberman et al. 1990). Recently, postsynaptic patch-clamp recordings were made from type II fibers in rats (Weisz et al. 2009). Although type II fibers receive input from a large number of $\mathrm{OHCs}$, the observed postsynaptic activity was far less frequent and generally smaller in amplitude as compared to similar postsynaptic recordings from type I fibers that receive their input from a single IHC active zone (Glowatzki and Fuchs 2002). Though it is speculated that type II fibers may mediate responses to loud, painful sounds or play a role in cochlear development, their exact role remains unclear.

Similar to the afferent system, IHCs and OHCs are also differentially innervated by descending, olivocochlear fibers. The following findings are based on experiments performed in cats and rodents (Liberman et al. 1990, Maison et al. 2003). OHCs are directly innervated by myelinated fibers mainly originating from the medial portion of the contralateral superior olivary complex (MSO). These fibers are thus termed the medial olivocochlear bundle (MOC). The MOC primarily uses acetylcholine (ACh) as neurotransmitter. IHCs, in contrast, are only tran- 
siently innervated by cholinergic efferents during development. Both IHCs and OHCs are hyperpolarized by the respective cholinergic efferents, via activation of small conductance calcium-activated $\mathrm{K}^{+}$channels (SK channels) that in turn is triggered by $\mathrm{Ca}^{2+}$ influx through nicotinic ACh receptors containing $\alpha 9 \alpha 10$-subunits (Glowatzki and Fuchs 2000, Oliver et al. 2000, Elgoyhen et al. 2001). Later in development, the more numerous, unmyelinated fibers of the lateral olivocochlear bundle (LOC) that mainly descend from the ipsilateral lateral superior olive (LSO) innervate the peripheral processes of type I ANFs beneath IHCs. LOC fibers are cytochemically more diverse, with evidence for ACh, $\gamma$-amino-butyric acid (GABA), dopamine (DA), and peptide neurotransmitters (Eybalin 1993). For afferent type I ANF fibers, there seems to be no tonotopic gradient in regard to their innervation by efferent LOC fibers (Liberman et al. 1990). The target of the MOC, the OHCs, suggests a function in the regulation of the gain of the cochlear amplifier, although there may be other, non-mechanical effects of MOC fiber activation. Notably, MOC activation reduces the spontaneous activity of type I ANFs. In contrast, less is known about the functions of the LOC, although it has been suggested to be involved in matching the sensitivities of the two ears, a prerequisite for accurate sound localization, (Darrow et al. 2006) and in preventing damage to the cochlea caused by intense acoustical stimulation (Darrow et al. 2007). It has also been suggested that cholinergic LOC fibers cause a slow increase in ANF response, whereas dopaminergic LOC fiber activation would lead to a suppression of firing rate in ANFs (Ruel et al. 2001, Groff and Liberman 2003). In contrast to the tonotopic gradient of OHC afferent innervation (see above), efferent innervation density of OHCs by MOC fibers decreases from base to apex (Liberman et al. 1990).

The third system, the adrenergic sympathetic input to the cochlea is studied less well (Eybalin 1993). The sympathetic fibers seem to contact cochlear blood vessels, but also the peripheral process of type I ANF axons in the region of the habenula perforata, where myelinization stops (Arnold 1974). While some function in control of cochlear blood flow seems likely, no data is available on the function of the ANF-contacting population (Eybalin 1993).

\subsubsection{Inner Hair Cells}

The pear-shaped inner hair cells are the principal sensory cells of the auditory modality. They are secondary sensory cells. The hair bundle of an IHC is formed by about 60 stereocilia that are arranged in 3 rows (Fig. 4). The basal pole of IHCs contains the neurotransmitter release 
machinery at several distinct presynaptic sites; the number of these sites varies between 10 to 30 and depends on cochlear position (Slepecky 1996). Like cones and rods in the retina, IHCs do not fire $\mathrm{Na}^{+}$-mediated action potentials. Instead the release of glutamate (Seal et al. 2008) at the presynaptic active zones is modulated by graded membrane potential changes (Goutman and Glowatzki 2007, Glowatzki et al. 2008) through a signal cascade involving $\mathrm{Ca}^{2+}$ influx through voltage-gated $\mathrm{Ca}^{2+}$ channels, which is shaped trough buffering by proteinaceous $\mathrm{Ca}^{2+}$ chelators, and subsequent $\mathrm{Ca}^{2+}$ binding to the vesicular $\mathrm{Ca}^{2+}$ sensor of exocytosis.

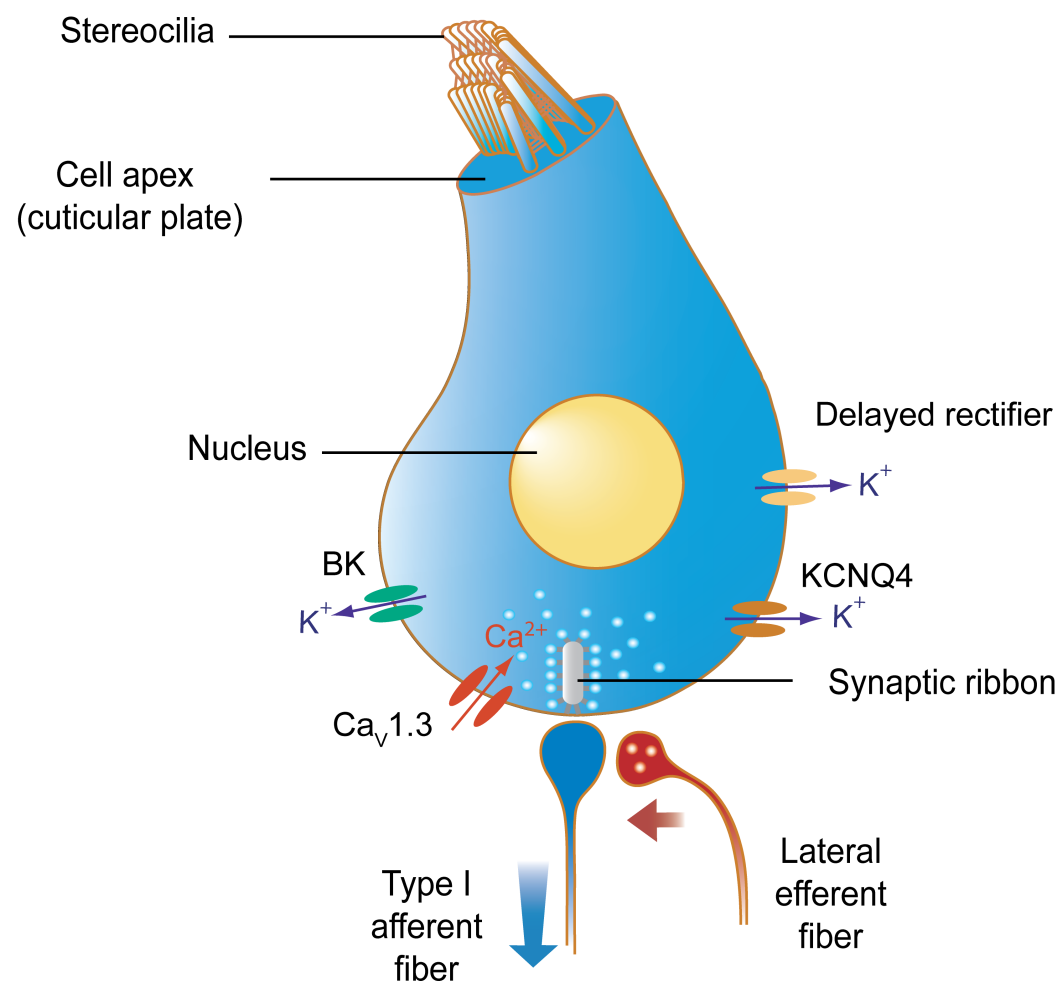

Figure 4 | Schematic representation of a mature IHC. The cartoon depicts the hair bundle at the apical pole of the IHC as well as the voltage-gated conductances present after the onset of hearing (3 types of $\mathrm{K}^{+}$conductances (BK, delayed rectifier, and $\mathrm{KCNQ} 4$ ), and L-type $\mathrm{Ca}^{2+}$ channels of the $\mathrm{Ca}_{\mathrm{V}} 1.3$ type). It also shows an exemplary synaptic contact of the IHC onto a type I auditory nerve fiber and the innervation of the postsynaptic dendrite by an efferent fiber from the lateral olivocochlear bundle (LOC). See text for further details.

\section{Voltage-gated $\mathrm{Ca}^{2+}$ Channels}

Depolarization increases the $\mathrm{p}_{\text {open }}$ of voltage-gated $\mathrm{Ca}^{2+}$ channels (VGCCs) that cluster at presynaptic active zones (Roberts et al. 1990, Issa and Hudspeth 1994, Stanley 1997, Brandt et al. 2005). IHCs display dihydropyridine (DHP)-sensitive $\mathrm{Ca}^{2+}$ currents that have fast activation kinetics, activate at comparably negative potentials (between -60 and $-50 \mathrm{mV}$ ), and exhibit very little inactivation (e.g. Cui et al. 2007), rendering them suitable to support sustained exocytosis. More than $90 \%$ of the VGCCs in IHCs are of the Ca 1.3 type $\left(\alpha_{1} \mathrm{D}\right.$; Platzer et al. 
2000, Brandt et al. 2003), while the remaining current is probably carried by a different type of L-type ( $\left.\mathrm{Ca}_{\vee} 1.4\right)$, as well as R-type ( $\left.\mathrm{Ca}_{\vee} 2.3\right)$ channels (Brandt et al. 2003), although the molecular identity of the latter two has not yet been confirmed. Consequently, mice lacking the $\mathrm{Ca}_{\mathrm{V}} 1.3 \alpha_{1}$-subunit are congenitally deaf (Platzer et al. 2000), due to failure of IHC exocytosis (Brandt et al. 2003). Functional VGCCs are supra-molecular complexes in which the pore-forming $\alpha_{1}$-subunit is associated with auxiliary $\beta$-, $\gamma$-, and $\alpha_{2} \delta$-subunits (Catterall 2000). These subunits can differentially regulate both the gating and the membrane targeting of VGCCs. In IHCs, for instance, the $\beta_{2}$ subunit is required for efficient surface expression of $\mathrm{Ca}_{\vee} 1.3$ channels and normal hearing (Neef et al. 2009). Fluctuation analysis on $\mathrm{Ca}^{2+}$ currents indicates that mature apical IHCs in mice contain on average about 1,700 VGCCs (Brandt et al. 2005). A similar number was found in frog saccular hair cells (Roberts et al. 1990); however, there may be transiently more $\mathrm{Ca}^{2+}$ channels expressed in immature IHCs (Beutner and Moser 2001, Brandt et al. 2003, Johnson et al. 2005, Zampini et al. 2010). Assuming an extrasynaptic density of 1 channel per square micron, the average number of $\mathrm{Ca}_{\mathrm{V}} 1.3$ channels per active zone was thus estimated to be $\approx 80$ in mature apical IHCs (Brandt et al. 2005; see also chapter 3).

\section{$\mathrm{Ca}^{2+}$ Influx, $\mathrm{Ca}^{2+}$ Buffering, and $\mathrm{Ca}^{2+}$ Homeostasis}

Even in the absence of sound, 'spontaneous' activity can be recorded from type I ANFs (Kiang et al. 1965). There is good evidence that this spontaneous activity is triggered by transmitter release from IHCs and is dependent on VGCC activity (Robertson and Paki 2002). Thus, a fraction of synaptic VGCCs seems to be open in IHCs 'at rest'. Parallel to this 'resting' $\mathrm{Ca}^{2+}$ influx at synapses, $\mathrm{Ca}^{2+}$ ions may continuously enter IHCs through open MET channels (Lumpkin and Hudspeth 1995). In order to avoid accumulation of $\mathrm{Ca}^{2+}$ ions and cross-talk between the different functional compartments of an $\mathrm{IHC}, \mathrm{Ca}^{2+}$ has to be constantly removed from the cytosol. In mouse IHCs, this task seems to be chiefly accomplished by plasma membrane ATP-driven $\mathrm{Ca}^{2+}$ pumps (Kennedy 2002). Additionally, thapsigarginsensitive intracellular stores as well as mitochondria supposedly contribute to intracellular $\mathrm{Ca}^{2+}\left(\left[\mathrm{Ca}^{2+}\right]_{\mathrm{i}}\right)$ homeostasis, at least in immature IHCs (Kennedy 2002). While these mechanisms regulate background cytosolic $\left[\mathrm{Ca}^{2+}\right]_{\mathrm{i}}$, they may not be fast and/or their respective effectors not localized close enough to synaptic $\mathrm{Ca}^{2+}$ influx sites to rapidly terminate spatially restricted, large $\left[\mathrm{Ca}^{2+}\right]_{\mathrm{i}}$ increases, as mediated by synaptic $\mathrm{Ca}^{2+}$ channel clusters (Roberts 1994). This, however, is likely demanded by the temporal characteristics of auditory stimuli (Palmer and Russell 1986). IHCs are therefore equipped with mobile proteinaceous $\mathrm{Ca}^{2+}$ buffers, par- 
valbumin- $\alpha$, calretinin and calbindin (Hackney et al. 2005). Their concentrations as well as their $\mathrm{Ca}^{2+}$ association and dissociation kinetics play a major role in determining both the amplitude and the spatial spread of the presynaptic $\mathrm{Ca}^{2+}$ signals that govern neurotransmitter release (Neher and Augustine 1992, Roberts 1993, Edmonds et al. 2000).

Together, the clustering of VGCCs at active zones and the diffusion-limiting actions of buffers establish domains of locally elevated $\left[\mathrm{Ca}^{2+}\right]_{\mathrm{i}}$. For any (particularly low-affinity) effector of $\left[\mathrm{Ca}^{2+}\right]_{i}$, like the vesicular $\mathrm{Ca}^{2+}$ sensor for fusion, their spatial relation to these domains is of considerable functional significance. If, for instance, the $\mathrm{Ca}^{2+}$ sensor is located very close to a $\mathrm{Ca}^{2+}$ channel $(\approx 20 \mathrm{~nm})$, it will be readily exposed to large $\left[\mathrm{Ca}^{2+}\right]_{\mathrm{i}}$ excursions of several tens to hundreds of $\mu \mathrm{M}$ once the channel opens (Roberts 1994). This region in the very vicinity of the channel mouth is often referred to as 'nanodomain' (Neher 1998). Experimentally, it can be characterized by the fact that only very 'fast' buffers (high association rate, $k_{\text {on }}$; e.g. 1,2bis(2-aminophenoxy)ethane- $N, N, N 9, N 9$-tetraacetate (BAPTA); Naraghi 1997) are effective in capturing $\mathrm{Ca}^{2+}$ ions before they reach the $\mathrm{Ca}^{2+}$ sensor - and hence in inhibiting its function. One immediate implication for a vesicular $\mathrm{Ca}^{2+}$ sensor with 'nanodomain' location is the ability to act rapidly upon channel opening, reflected in exocytic delays in the range of hundreds of microseconds (Yamada and Zucker 1992).

If the $\mathrm{Ca}^{2+}$ sensor, in contrast, is placed farther away from the channel $(\approx 100-200 \mathrm{~nm})$, it will experience smaller $\left[\mathrm{Ca}^{2+}\right]_{\mathrm{i}}$ elevations, a longer time to peak $\left[\mathrm{Ca}^{2+}\right]_{i}$, and an increased susceptibility towards buffering, also by 'slow' buffers (lower association rate, $\mathrm{k}_{\mathrm{on}}$; e.g. ethylene glycol-bis-(2-aminoethyl)-N,N,N',N'-tetraacetic acid (EGTA); Naraghi 1997). This regime of the $\left[\mathrm{Ca}^{2+}\right]_{\text {i }}$ profile around the $\mathrm{Ca}^{2+}$ sensor is termed 'microdomain' (Neher 1998, Augustine et al. 2003). If, however, several channels in a cluster open more or less simultaneously, 'their' individual $\left[\mathrm{Ca}^{2+}\right]_{\mathrm{i}}$ clouds may overlap and the summed signal at the distant site of the $\mathrm{Ca}^{2+}$ sensor may be large in amplitude. At the same time, the delay to reach a given $\left[\mathrm{Ca}^{2+}\right]_{\mathrm{i}}$ will be shorter. Thus, rapid exocytosis is also achievable with a microdomain control of transmitter release - as it is the case at the calyx of Held synapse (Borst and Sakmann 1996). Nonetheless, activation of the $\mathrm{Ca}^{2+}$ sensor will still be sensitive to the action of 'slow' buffers, as the distance between the channel mouth and the sensor is not small enough to be readily bridged by the diffusion-limited $\mathrm{Ca}^{2+}$ signal without being intercepted by the buffer. Another functional difference between these two described, somewhat limit case scenarios relates to their response upon an increasing recruitment of open $\mathrm{Ca}^{2+}$ channels - i.e. a change in popen. While 
a linear increase in the response is predicted for a nanodomain location of a (low-affinity) $\mathrm{Ca}^{2+}$ sensor (apparent or $\mathrm{Ca}^{2+}$ channel cooperativity; Matveev et al. 2009), a supra-linear increase is expected for a microdomain placement of the sensor (reflecting its intrinsic or $\mathrm{Ca}^{2+}$ current cooperativity; Matveev et al. 2009). This is because - in the latter case - a certain number of $\mathrm{Ca}^{2+}$ channels has to open to reach the threshold $\left[\mathrm{Ca}^{2+}\right]_{\mathrm{i}}$ for a response in the first place, but as this threshold is approached more or less globally within the microdomain, it will affect many $\mathrm{Ca}^{2+}$ sensor molecules within a rather narrow range of further $\left[\mathrm{Ca}^{2+}\right]_{\mathrm{i}}$ increases.

There is good evidence that vesicle release at the hair cell ribbon synapse is under nanodomain control (Brandt et al. 2005, Keen and Hudspeth 2006, Goutman and Glowatzki 2007; but see: Roberts 1994, Tucker and Fettiplace 1995; for review: Moser et al. 2006).

Experimentally, $\mathrm{Ca}^{2+}$ influx through clusters of $\mathrm{Ca}^{2+}$ channels can be visualized by $\mathrm{Ca}^{2+}$ imaging techniques (see below). $\mathrm{Ca}^{2+}$ indicators have been used to investigate the associated localized high- $\left[\mathrm{Ca}^{2+}\right]_{\mathrm{i}}$-domains. These so called hot spots were first observed in axonal growth cones (Silver et al. 1990) but have been later found in various other preparations, amongst them hair cells of lower vertebrates (Issa and Hudspeth 1994, Tucker and Fettiplace 1995, Issa and Hudspeth 1996), and retinal bipolar cells (Zenisek et al. 2003). Interestingly, a study in immature mouse IHCs reported the absence of distinct hot spots, but rather a uniform elevation of $\left[\mathrm{Ca}^{2+}\right]_{\mathrm{i}}$ throughout the basal portion of the cells (Kennedy and Meech 2002), besides $\mathrm{Ca}^{2+}$ channel clustering in mature IHCs (Brandt et al. 2005). This discrepancy triggered interest in the question whether localized $\mathrm{Ca}^{2+}$ influx domains would be observed in mature IHCs - or whether the diffuse $\mathrm{Ca}^{2+}$ signals are independent of developmental stage (chapters 2,3).

However, while providing valuable information about localization, amplitude, kinetics, and regulation of local $\mathrm{Ca}^{2+}$ influx, current light microscopy techniques do not have the necessary resolution to directly reveal the presence or absence of nanodomains within the $\mathrm{Ca}^{2+}$ microdomains present at presynaptic active zones.

\section{Molecular Components of Transmitter Release}

To release their neurotransmitter content into the synaptic cleft, synaptic vesicles (SVs) have to be in close physical proximity to the presynaptic plasma membrane (Südhof 2004). Additionally to this requirement for docking, SVs have to assemble their vesicular release machinery in complementation with plasma membrane proteins to become fusion competent, or primed (molecular priming; Neher and Sakaba 2008). Eventually, fusion only happens if the 
$\left[\mathrm{Ca}^{2+}\right]_{\mathrm{i}}$ in vicinity of the (low-affinity) vesicular $\mathrm{Ca}^{2+}$ sensor is high enough, i.e. in the range of tens of $\mu \mathrm{M}$ (Beutner et al. 2001). As such high $\left[\mathrm{Ca}^{2+}\right]$ concentrations usually only exist in the close vicinity of $\mathrm{Ca}^{2+}$ channels (Roberts 1994), physiologically, fusion competence requires sufficient proximity of a SV to a $\mathrm{Ca}^{2+}$ source (positional priming; Neher and Sakaba 2008). Although significant progress has been achieved over the last 10-15 years in elucidating the molecular nature of some of the key players involved in the SV cycle at conventional synapses (Südhof 2004), increasing evidence accumulates that the afferent IHC synapse is devoid of several of these molecules identified at synapses of the central nervous system. For instance, IHC synapses seem to lack complexins (Strenzke et al. 2009) as well as the synaptotagmins I and II (Safieddine and Wenthold 1999; but see: Johnson et al. 2010). Instead, IHC synapses appear to at least partly utilize a different set of proteins, e.g. the large multi-C2domain protein otoferlin (Roux et al. 2006), and the unconventional vesicular glutamate transporter 3 (Seal et al. 2008, Ruel et al. 2008), possibly due to the different functional requirements imposed onto the synaptic machinery.

\section{Synaptic Transfer Function, Multivesicular Release, and Vesicle Recycling}

One such distinctive property of the afferent IHC synapse is the predominance of multivesicular release (MVR; Glowatzki and Fuchs 2002). MVR, however, is also observed at other ribbon-type synapses (Singer et al. 2004) and non-ribbon-type synapses (He et al. 2009). Interestingly, in IHCs, depolarization seems to have an effect on the frequency of excitatory postsynaptic currents (EPSCs), but not on their amplitude (Glowatzki and Fuchs 2002). This observation, however, may not be generally applicable to all types of hair cells across different species (e.g. Li et al. 2009). Until now, it is not resolved how MVR is mechanistically implemented, although exocytosis of pre-fused SVs (compound fusion), synchronized fusion of single SVs, or serial homotypic SV fusion (cumulative fusion) are among the potential mechanisms under discussion (Neef et al. 2007). Neither has the functional significance of MVR been elucidated yet. The postsynaptic boutons of type 1 ANFs are small $(<1 \mu \mathrm{m})$ and have a high input resistance (G $\Omega$-range; Glowatzki and Fuchs 2002). It is thus expected that already small currents would lead to excitatory postsynaptic potentials (EPSPs) of substantial amplitude. Indeed, sharp electrode recordings from peripheral type I ANF processes revealed that nearly all EPSPs were of sufficient size to trigger an action potential (AP), at least in a subset of ANFs (high-spontaneous rate ANFs; see below) and the ones that failed likely did so because of ANF refractoriness (Siegel 1992). Although it thus seems that even small EPSPs can trigger APs, MVR may help to explain the observed low rate of postsynaptic failures. Besides, 
MVR may decrease temporal jitter in AP generation, as large EPSPs reach the threshold for AP initiation earlier (Trussell 2002). Additionally, the observation of variations in the degree of synchronized MVR between type I ANFs (Singer et al. 2009) could possibly explain observed differences in ANF discharge rates (Kiang et al. 1965; see below). The continuous fusion of SVs with the plasma membrane in IHCs, however, imposes a strong need for both sufficient re-supply of SVs as well as removal of the inserted membrane. IHC synapses, like photoreceptor synapses, harbor an electron-dense osmiophilic structure at their presynaptic active zone, called the synaptic ribbon or synaptic body (Lenzi and von Gersdorff 2001, Schmitz 2009). IHC synapses typically have a single synaptic ribbon at their active zones (Khimich et al. 2005). In electron micrographs, these ribbons appear usually surrounded by a halo of SVs, tethered to the ribbon by thin, short $(\approx 20 \mathrm{~nm})$ linkers (Lenzi et al. 1999). The exact function(s) of the synaptic ribbon has (have) largely remained elusive. Proposed roles include the facilitation of compound fusion (Matthews and Sterling 2008), a function in efficient vesicle re-supply (Lenzi and von Gersdorff 2001), or a role in the stabilization of a large pool of release-ready SVs (Khimich et al. 2005). In order to explain the rapid re-supply of IHC active zones with SVs during and after stimulation it has also been suggested that large cytoplasmic compartments in the apical compartment of IHCs generate SVs that subsequently travel to the active zones in the basal portion of IHCs where they sustain exocytosis (Griesinger et al. 2005). Finally, endocytosis was reported to take place in the vicinity, but outside of the presynaptic active zone (Lenzi et al. 1999), as well as at the apical pole of IHCs (Griesinger et al. 2005). However, IHCs seem to also employ at least two different modes of endocytosis, a slow, $\left[\mathrm{Ca}^{2+}\right]_{\mathrm{i}}$-independent mode and a second, faster mode which is stimulated by $\left[\mathrm{Ca}^{2+}\right]_{\mathrm{i}}$ (Beutner et al. 2001).

\section{Further Ionic Conductances of IHCs}

In addition to mechanotransducer channels and synaptic VGGCs, IHCs exhibit a set of other voltage-gated conductances to meet the functional demands placed upon them (Fig. 4). In this respect it is noteworthy that during development, IHCs undergo substantial changes in ion channel composition, innervation pattern, and synaptic transmission (Beutner and Moser 2001, Housley et al. 2006). After the onset of hearing at around post-natal day (P)12 in mice (Ehret 1985), IHCs express three types of voltage-gated $\mathrm{K}^{+}$channels (Housley et al. 2006). The first class, Large-conductance $\mathrm{Ca}^{2+}$-activated $\mathrm{K}^{+}(\mathrm{BK})$ channels, are thought to carry a fast activating $\mathrm{K}^{+}$current $\left(\mathrm{I}_{\mathrm{K}, \mathrm{f}}\right)$ that prevents initiation of $\mathrm{Ca}^{2+}$-mediated APs (Kros et al. 1998), which are observed in pre-mature IHCs (Beutner and Moser 2001, Marcotti et al. 2003, 
Tritsch et al. 2007). Due to their large conductance, opening of BK channels reduces the IHC's input resistance, which leads to a dramatic reduction of the membrane time constant, an adaptation crucial to the function of IHCs as high-frequency signal transducers (Kros et al. 1998). Interestingly and in contrast to lower vertebrates, BK channels seem to be largely decoupled from VGCCs in mammalian IHCs (Kros and Crawford 1990, Thurm et al. 2005). Therefore, BK activation can actually precede the opening of VGCCs during very rapid depolarizations. The second $\mathrm{K}^{+}$conductance is mediated by a slowly activating, 4-aminopyridinesensitive delayed rectifier current $\left(\mathrm{I}_{\mathrm{K}, \mathrm{s}}\right)$ (Kros and Crawford 1990). It is also implicated in shaping the receptor potential of IHCs, and to a small degree, their resting potential. Additionally, adult IHCs also express KCNQ4 channels (Oliver et al. 2003). The corresponding inwardly rectifying conductance $\left(\mathrm{I}_{\mathrm{K}, \mathrm{n}}\right)$ has a negative activation range, is open around the resting membrane potential of isolated IHCs $(-70 \mathrm{mV})$, and has been hypothesized to hence influence the resting membrane potential (Oliver et al. 2003), although in vivo IHCs may be more depolarized at rest (Dallos 1985). Immature IHCs $(<\mathrm{P} 12)$ transiently as well express voltage-gated $\mathrm{Na}^{+}$channels and (voltage-insensitive) small-conductance $\mathrm{Ca}^{2+}$-activated $\mathrm{K}^{+}$ channels (Housley et al. 2006; see above).

\section{Hair Cell Function along the Tonotopic Axis}

The fact that hair cells along the tonotopic axis of the cochlea (see above) respond to a large range of different sound frequencies raises the question whether they show functional specializations in dependence on cochlear location. Studies on hair cells in hearing end-organs of non-mammalian vertebrates have indeed reported several differences in various important parameters of hair cell physiology, such as mechanotransduction (Ricci 2002, Ricci et al. 2003), $\mathrm{Ca}^{2+}$ buffering (Ricci et al. 2000, Hackney et al. 2003), $\mathrm{Ca}^{2+}$ influx (Martinez-Dunst et al. 1997, Ricci et al. 2000, Schnee and Ricci 2003), and exocytosis (Schnee et al. 2005, Rutherford and Roberts 2006) as a function of position along the tonotopic map. However, the low characteristic frequencies of the hair cells in most of these studies limit their validity in providing clues about potential adaptations to frequencies larger than $1 \mathrm{kHz}$. Moreover, hair cells in many lower vertebrates rely substantially on electrical tuning mechanisms, intrinsic to their membrane, to gain frequency selectivity (Fettiplace and Fuchs 1999). In contrast, frequency selectivity in the mammalian cochlea is determined by the micromechanics of the cochlear partition and the electromechanical feedback of the cochlear amplifier (see above). Consequently, intrinsic differences in IHC function along the mammalian cochlea do not have to be invoked to explain the narrow frequency tuning seen in ANF recordings from the coch- 
lea (Liberman 1982a). Nonetheless, variations of fundamental biophysical properties of IHCs have been observed also in the mammalian cochlea, including differences in $\mathrm{Ca}^{2+}$ buffering (Hackney et al. 2005), and synaptic exocytosis (Johnson et al. 2008). However, many questions about a potential specialization of IHC function towards the most sound-sensitive, midcochlear regions were not addressed in these studies - and hence remain unclear. Additionally, data on differences in presynaptic function of IHCs within a given cochlear location are also lacking. We thus set out to investigate these aspects of cochlear function in the mouse cochlea, by using $\mathrm{Ca}^{2+}$ imaging and whole-cell patch-clamp techniques (see chapter 3 ).

\subsubsection{Auditory Nerve Fibers}

Sound is encoded by the interplay of IHCs and spiral ganglion neurons. While the spiral ganglion neurons are driven by transmitter release from IHCs, their axons - forming the auditory nerve - send the sensory information in form of spike trains from the peripheral auditory to the central nervous system.

\section{Postsynaptic Receptors}

Postsynaptically, glutamate depolarizes ANF type I terminals via activation of $\alpha$-amino-3hydroxy-5-methyl-4-isoxazolepropionic acid (AMPA) receptors (Glowatzki and Fuchs 2002). Recent data suggests that the AMPA receptors are organized into a ring-like structure in the afferent terminal (chapter 3), maybe to increase efficiency of glutamate detection. Lately, it has also been proposed that type I ANF terminals additionally express N-methyl-D-aspartate (NMDA) receptors, the latter not being involved in fast, 'regular' afferent synaptic transmission but only contributing to glutamate induced postsynaptic responses in the presence of elevated levels of the NMDA receptor regulator arachidonic acid (Ruel et al. 2008).

\section{Glutamate Clearance}

ANFs support instantaneous firing rates of up to $1 \mathrm{kHz}$ as well as adapted rates of several hundred Hz (Liberman 1978, Taberner and Liberman 2005). They also exhibit phase-locking - i.e. the preferential occurrence of APs during a certain phase of the stimulus - up to sound frequencies of several kHz (Palmer and Russell 1986, Taberner and Liberman 2005). The underlying high-frequency synaptic transmission requires both rapid build-up and termination of signals in order to avoid smearing of subsequent responses. This principle applies to presynaptic $\left(\left[\mathrm{Ca}^{2+}\right]_{\mathrm{i}}\right.$ signals, see above) as well as postsynaptic stages (e.g. AMPA receptor occu- 
pancy). Tightly connected to the latter is the removal of neurotransmitter from the synaptic cleft. Rapid diffusion from the narrow cleft followed by dilution in the extracellular space and uptake by glutamate transporters into neighboring supporting cells seems to avoid accumulation of glutamate within the cleft. This arrangement would circumvent potential problems with glutamate transporter saturation due to their high-affinity to glutamate and low efficiency in transport (Glowatzki et al. 2006). Consistently, no glutamate transporters were found in IHCs or postsynaptic boutons of type I ANFs. Interestingly, glutamate excitotoxicity seems to play an important role in noise-induced hearing loss (Ruel et al. 2000).

\section{Response Characteristics of Auditory Nerve Fibers}

The following section will deal with the response characteristics of type I ANFs, as very little data is available on type II ANFs (see above). Consistent with the fact that type I ANFs receive input from a single IHC, they exhibit sharp frequency-tuning. This means that a very narrow band of frequencies is able to elicit an increase in firing rate above spontaneous rate at considerably lower stimulus intensities than the remaining frequency bands (Liberman 1982a, Taberner and Liberman 2005). The frequency with the highest sensitivity is called characteristic frequency $(\mathrm{CF})$. ANFs are usually characterized by their response to tone bursts or short bursts of broad-band noise. For this purpose, the stimulus is repeatedly presented to the (usually anaesthetized) animal, while the spiking activity in the ANF is recorded. From the entity of recorded responses, a post-stimulus time histogram (PSTH) is assembled, in which the number (or rate) of spikes is plotted as a function of time. Thus, the PSTH reflects the probabilities of spike occurrence at the respective time point. The basic shape of the PSTH is similar for all ANFs across different animals: There is an initial high rate of firing (up to $1 \mathrm{kHz}$ instantaneous firing rate; 'peak rate'), followed by an (usually bi-) exponential decay of the response to a steady-state ('adapted') rate (in most cases between 200 and 300 spikes per second; Liberman 1978, Taberner and Liberman 2005; see also Fig. A5 (appendix)). After the cessation of the stimulus, the firing rate drops below the spontaneous rate (SR; the fiber's firing rate in silence), and recovers exponentially to SR within approximately 50 to 100 milliseconds.

Although all ANFs exhibit this basic spiking pattern, they show pronounced differences in their fundamental response characteristics, for instance their threshold of activation, their dynamic range (DR), and their SR (Liberman 1978, Taberner and Liberman 2005). The dynamic range of a fiber describes the range of sound intensities over which a change in (average) fir- 
ing rate is measurable. The corresponding rate-level functions of most fibers seem to be described well by a sigmoidal function. DR is often defined in terms of the $10-90 \%$ range, i.e. the stimulus levels over which the fiber shows between 10 and $90 \%$ of its maximum response. While there is no clear relationship between SR and characteristic frequency (CF) of a given fiber, there is a negative correlation between SR on the one hand, and both DR and threshold on the other hand. Thus, high-SR ANFs usually show low thresholds and narrow DRs, i.e. they represent the most sensitive fibers which also saturate 'quickly' with rising sound intensities (Taberner and Liberman 2005). Their abundance seems to differ between species. While high-SR fibers constitute about $60 \%$ of the measured ANFs in cat (Liberman 1978), their relative contribution to the ANF fiber population appears smaller in mouse (Taberner and Liberman 2005). It is hypothesized that the auditory system collectively uses the information contained in these different 'loudness' channels to encode the wide range of sound intensities (about $120 \mathrm{~dB}$ ) that it is able to discriminate. It should be noted, however, that sound intensity coding is a very complex process that is far from being understood in all detail (Viemeister 1988, Colburn et al. 2003). While it was suggested that sufficient information for robust intensity coding over a wide dynamic range is present in the average ANF firing rates within a narrow frequency region, the stochastic nature of ANF firing impose limitations that decrease the performance of the auditory system (Colburn et al. 2003). In this respect it is interesting to note that recently observations of fast stimulus-dependent modulations of the input-output functions of auditory neurons have been described (Dean et al. 2005, Wen et al. 2009). While these modulations were much more pronounced at higher levels in the auditory system (inferior colliculus; Dean et al. 2005), they were also reported at the stage of ANFs (Wen et al. 2009).

It is believed that one IHC is presynaptic to ANFs of different axonal firing characteristics (Liberman 1982b). This connectivity scheme would allow a single IHC to encode a wide range of sound intensities by utilizing an array of channels with different sensitivities (see above; chapters 2 and 3). It is an intriguing feature of this arrangement that the electrotonically compact IHC seemingly sends different aspects of information through a voltage sensitive pathway (chapter 2). However, the actual mechanisms behind the heterogeneity in ANF firing properties have not been identified, although several candidates are being considered. The following paragraphs outline the most prominent schemes. 


\section{Postsynaptic Candidate Mechanisms}

First, the postsynaptic ANFs could simply differ in the number and/or properties of AMPA receptors. While, according to this model, a given amount of transmitter would depolarize the high-AMPA-content ANF above threshold, the same amount of glutamate would fail to elicit a depolarization in the low-AMPA-content ANF. However, the expected outcome would be a scaling of spike-rates (both evoked and spontaneous) towards the asymptotic rates set by ANF refractoriness. This is not consistent with the observation that ANFs with different SRs have comparable adapted peak rates over a very broad range of SRs (Liberman 1978). Interestingly, ANFs of different SRs diverge in their morphology. While high-SR ANFs show thick (0.8$1.2 \mu \mathrm{m}$ diameter) and mitochondrion-rich peripheral processes, low-SR ANFs have thinner (0.8-0.3 $\mu \mathrm{m}$ diameter) and mitochondrion-poor processes (Liberman 1982b). Although a larger diameter and the resulting decrease in internal resistance increases the electrotonic spread of membrane potential perturbations, it is not clear, however, whether these differences actually cause the various ANF firing behaviors or if they rather reflect adaptations to the resultant different energetic needs. Additionally, differences in ANF firing behavior could also emanate from variations in active membrane properties. For instance, pronounced cell-to-cell heterogeneity - independent of CF (as found for some conductances, e.g. Adamson et al. 2002) - has been reported for the activation parameters of hyperpolarization-activated cationic $\left(\mathrm{I}_{\mathrm{h}}\right)$ current in ANFs (Mo and Davis 1997). Based on immuno-histochemical findings, inter-cell variability has also been reported for voltage-gated $\mathrm{K}^{+}$conductances in guinea pig ANFs (Bakondi et al. 2008).

\section{Efferent Candidate Mechanisms}

The efferent innervation of the peripheral type I ANF processes by the lateral olivo-cochlear bundle (LOC; see above), represents another potential candidate for the differential regulation of type I ANF firing. For instance, efferent synapses were found to be especially numerous on the thin peripheral processes of ANFs, which are supposed to present the morphological correlate of low-SR/high-threshold fibers (see above; Liberman 1980, Merchan-Perez and Liberman 1996). It thus could be hypothesized that a tonic inhibition of ANFs yields fibers with low SR and high thresholds. Indeed, evidence for a dopamine-mediated tonic inhibition of LOC fibers has been reported (Ruel et al. 2001). However, lesioning of the LOC did not alter the fundamental relation between SR and threshold (Liberman 1990), arguing against tonic inhibition being the mechanism behind SR differences. The observation that both an increase as well as a decrease in ANF firing rate can be elicited via the LOC pathway (Groff and Li- 
berman 2003), may be explained by the parallel existence of different transmitter systems in the LOC pathway (see above) and their further diversification by peptidergic co-transmitters (Eybalin 1993).

\section{Presynaptic Candidate Mechanisms}

Besides postsynaptic or efferent mechanisms, presynaptic specializations have also been discussed to explain the wide auditory nerve fiber firing characteristics,. As in most mammals each presynaptic active zone provides the exclusive sensory input to its postsynaptic ANF, the presynapse is indeed in a key position to determine the ANF firing behavior (Nadol 1988). Early evidence for a presynaptic mechanism came from electron-microscopy based observations that auditory nerve fibers of different properties receive input from presynaptic active zones with different morphologies (Merchan-Perez and Liberman 1996). This study showed, based on a small sample, that - at least in cats - low-spontaneous rate fibers are postsynaptic to active zones that tend to be larger and have larger synaptic ribbons with more synaptic vesicles. However, the restricted quantity of observations in that study leaves some degree of uncertainty regarding how generalizable these observations are. Moreover, it is currently unclear to which degree they apply to other mammals, and whether they are of physiological relevance in these animals (Francis et al. 2004). Another hypothesis regards the function of presynaptic $\mathrm{Ca}^{2+}$ channels. Supported by computer simulations, it has been suggested that differences in the presynaptic $\mathrm{Ca}^{2+}$ conductance between active zones could differentially control the ANF responses (Sumner et al. 2002). However, no direct observations of such differences had been reported. Furthermore, it has also been hypothesized that inter-synaptic differences in the degrees of multivesicular release could yield a broad spectrum of different EPSP (excitatory postsynaptic potentials) amplitude distributions and thus differentially regulate action potential generation in the respective postsynaptic ANF (Singer et al. 2009).

Due to the fact that a presynaptic mechanism would be located upstream of a potential postsynaptic or efferent ANF modulation, it would very likely not be affected by these other sites (Slepecky 1996) and allow to conclude about its relevance with the least degree of ambiguity. This and the key role of $\mathrm{Ca}^{2+}$ in the regulation of neurotransmitter release prompted us to compare presynaptic $\mathrm{Ca}^{2+}$ signals between single active zones of inner hair cells (chapters 2, 3). The recent observation of stimulus-dependent adaptations in ANF firing sensitivity (Wen et al. 2009; see above) further underlines the imperative of a better understanding of sound intensity encoding within the cochlea, especially at the level of IHC synapses. 


\subsection{Confocal $\mathrm{Ca}^{2+}$ Imaging}

Live-cell imaging provides the capability of selectively investigating cellular functions at subcellular resolution. Particularly suited for this purpose is the use of imaging techniques with optical sectioning capabilities such as one-photon confocal or multi-photon microscopy, as they allow the rejection or avoid the emission of light from planes above and below a location of potential interest, thereby greatly increasing the contrast of the signal (changes) under study. Given the availability of an adequate optical reporter one can track specific cellular processes at distinct locations with high temporal resolution and potentially at low levels of invasiveness. $\mathrm{Ca}^{2+}$ represents a second messenger that received wide interest in imaging studies - due to its manifold functions in most cell types as well as the availability of suitable reporters. Calcium indicators, in most cases, are fluorescent molecules that alter their photophysical properties upon binding of $\mathrm{Ca}^{2+}$ ions. Following the absorption of light of appropriate wavelength, they are excited and subsequently can relax from the excited singlet state $\left(\mathrm{S}_{1}\right)$ to the non-excited ground state $\left(\mathrm{S}_{0}\right)$ under the emission of fluorescent light. The exact energy difference between these two states determines the wavelength of the emitted fluorescent light. Calcium indicators can be subdivided in different groups according to the specific alterations they undergo upon $\mathrm{Ca}^{2+}$ chelation (Takahashi et al. 1999). One prominent group of indicators primarily exhibits a change in their excitation or emission peaks (ratiometric dyes, e.g. Fura-2). A second, large group of indicators reports changes in $\left[\mathrm{Ca}^{2+}\right]$ as a change in fluorescence intensity, without a significant shift in their excitation or emission spectra (nonratiometric or single-wavelength dyes, e.g. Fluo-5N).

In a conventional one-photon laser scanning confocal microscope (CLSM), one or several fluorophores are excited by a focused laser beam that is projected onto the specimen through the objective lens of the microscope. A fraction of the emitted photons is captured by the same lens, and travels the optical path also taken by the excitation light, to the primary (or excitation) dichroic device. The dichroic device separates the emission from the excitation light path. The emitted photons that remain after the 'costly' passage through the microscope are then usually detected by a sensitive point detector (such as a photomultiplier tube (PMT) or a photodiode) that is located behind a small aperture. This pinhole prevents light that originated from sections above and below the focal plane from reaching the detector. Thereby, confocal microscopy allows optical sectioning of specimens, permits visualisation of localized signals without their degradation by fluorescence from neighbouring planes, and generally improves image contrast by rejection of this out-of-focus light from the detector. While the 
construction of 1- or 2-dimensional images requires scanning of the focused laser beam across the sample (and thus limits temporal resolution of spatially resolved 'standard' confocal microscopy), the readout of fluorescence from a single location (with a static laser beam) offers very high temporal resolution, however at the cost of spatial dimensionality.

Immanent to the confocal design is the rejection of photons. While the largest fraction of the rejected photons will not originate from the focal plane if the pinhole is opened to $\approx 1$ Airy unit (i.e. the size of the innermost ring of a point-source-derived diffraction pattern (Airy disk) at the secondary image plane, where the pinhole is located), some photons from the focal plane will be missed (the remaining photons from the outer rings of the Airy disk). For that reason, confocal microscopy may reject not only unwanted, but also relevant signal, albeit at low amounts. Another potential problem, imposed by the use of laser light sources and high numerical aperture objectives, are effects of photobleaching and phototoxicity. In order to minimize the damage imposed onto the cell as well as onto the indicator, appropriate illumination settings have to be chosen.

\subsection{Aim of the Work and Overview}

We used fast confocal $\mathrm{Ca}^{2+}$ imaging in conjunction with whole-cell patch clamp recordings to address the 4 main points of this work, including a (i) general description of presynaptic $\mathrm{Ca}^{2+}$ signals at mature mouse IHC ribbon synapses, (ii) a characterization of their heterogeneity in light of a potential role in sound intensity coding, (iii) a description of tonotopic differences in both whole-cell and synaptic $\mathrm{Ca}^{2+}$ influx in IHCs, and (iv) a characterization of the dependence of presynaptic $\mathrm{Ca}^{2+}$ signal heterogeneity on both tonotopic and intracellular location. The experimental work was backed and supplemented by a $\mathrm{Ca}^{2+}$ reaction-diffusion-imaging model, partly based on publicly available software (Matveev et al. 2002). A general description of presynaptic $\mathrm{Ca}^{2+}$ microdomain signals, alongside with an investigation of their main determinants is presented in chapter 2. The same chapter also deals with the finding of marked $\mathrm{Ca}^{2+}$ microdomain heterogeneity in both amplitude and voltage-dependence, and presents results supporting the idea of $\mathrm{Ca}^{2+}$ channel number as a mechanism behind the observed $\mathrm{Ca}^{2+}$ microdomain amplitude variability. Chapter 3 contains a detailed biophysical characterization of whole-cell $\mathrm{Ca}^{2+}$ influx in IHCs at two different positions along the cochlea, showing that the number of $\mathrm{Ca}^{2+}$ channels is higher, and consequently $\mathrm{Ca}^{2+}$ current amplitude larger, in a mid-cochlear as compared to the apical region of the cochlea. This increase in $\mathrm{Ca}^{2+}$ channel 
number co-varies with synapse number and cochlear sensitivity. Chapter 3 moreover describes highly similar (average) presynaptic $\mathrm{Ca}^{2+}$ signals at the two cochlear locations, but reports pronounced amplitude heterogeneity within both cochlear regions. Additionally, it provides evidence for a loose segregation of functionally different synapses around the perimeter of mouse IHCs. Potential experimental artefacts are carefully addressed throughout both chapters, as well as in the appendix, both by experimental and theoretical means. Chapter 4, eventually, summarizes the work, outlines consistency between experiments and with the literature, and addresses remaining open questions and alternative hypotheses. 


\title{
2. Mechanisms Contributing to Synaptic $\mathrm{Ca}^{2+}$ Signals and Their Heterogeneity in Hair Cells.
}

\author{
Frank, T., Khimich, D., Neef, A., and Moser, T. (2009) \\ Proc Natl Acad Sci U S A, 106:4483-8 \\ http://www.pnas.org/content/106/11/4483.long \\ doi: $10.1073 /$ pnas.0813213106
}

Author contributions (as appearing in the paper):

T.F. and T.M. designed research; T.F. and D.K. performed research; T.F. and A.N. analyzed data; and T.F., A.N., and T.M. wrote the paper.

Detailed author contributions of T. Frank:

- Experimental work (excluding immuno-histochemistry shown in Fig. 5C, E; done by D. Khimich)

- Analysis (excluding analysis of immuno-histochemistry shown in Fig. 5C, E; done by A. Neef)

- $\mathrm{Ca}^{2+}$ reaction-diffusion-imaging simulations

- Preparation of the manuscript (together with T. Moser and partly A. Neef)

\section{Copyright notice:}

This material is reproduced by permission from the National Academy of Sciences of the United States of America in accordance to their copyright and licensing regulations. (C) owned by the authors, 2009 . 


\title{
Mechanisms contributing to synaptic $\mathrm{Ca}^{2+}$ signals and their heterogeneity in hair cells
}

\author{
Thomas Frank ${ }^{a, b}$, Darina Khimicha, Andreas Neefc, and Tobias Mosere,b,c,1 \\ annerEarLab, Department of Otolaryngology and Center for Molecular Physiology of the Brain, University of Göttingen, 37099 Göttingen, Germany; \\ bInternational Max Planck Research School for Neurosciences, Göttingen Graduate School for Neuroscience and Molecular Biosciences, 37077 Göttingen, \\ Germany; and 'Bernstein Center for Computational Neuroscience, University of Göttingen, 37073 Göttingen, Germany
}

Communicated by A. James Hudspeth, The Rockefeller University, New York, NY, December 25, 2008 (received for review October 30, 2008)

\begin{abstract}
Sound coding at hair cell ribbon synapses is tightly regulated by $\mathrm{Ca}^{2+}$. Here, we used patch-clamp, fast confocal $\mathrm{Ca}^{2+}$ imaging and modeling to characterize synaptic $\mathrm{Ca}^{2+}$ signaling in cochlear inner hair cells (IHCs) of hearing mice. Submicrometer fluorescence hotspots built up and collapsed at the base of IHCs within a few milliseconds of stimulus onset and cessation. They most likely represented $\mathrm{Ca}^{2+}$ microdomains arising from synaptic $\mathrm{Ca}^{2+}$ influx through $\mathrm{Ca}_{v} 1.3$ channels. Synaptic $\mathrm{Ca}^{2+}$ microdomains varied substantially in amplitude and voltage dependence even within single IHCs. Testing putative mechanisms for the heterogeneity of $\mathrm{Ca}^{2+}$ signaling, we found the amplitude variability unchanged when blocking mitochondrial $\mathrm{Ca}^{2+}$ uptake or $\mathrm{Ca}^{2+}$-induced $\mathrm{Ca}^{2+}$ release, buffering cytosolic $\mathrm{Ca}^{2+}$ by millimolar concentrations of EGTA, or elevating the $\mathrm{Ca}^{2+}$ channel open probability by the dihydropyridine agonist BayK8644. However, we observed substantial variability also for the fluorescence of immunolabeled $\mathrm{Cav}_{\mathrm{v}} 1.3 \mathrm{Ca}^{2+}$ channel clusters. Moreover, the $\mathrm{Ca}^{2+}$ microdomain amplitude correlated positively with the size of the corresponding synaptic ribbon. Ribbon size, previously suggested to scale with the number of synaptic $\mathrm{Ca}^{2+}$ channels, was approximated by using fluorescent peptide labeling. We propose that IHCs adjust the number and the gating of Cav1.3 channels at their active zones to diversify their transmitter release rates.
\end{abstract}

calcium microdomain | coding | imaging | ribbon synapse | modeling

$\mathbf{H}$ air cells transform mechanical stimuli into glutamate release at their ribbon-type synapses (reviewed in refs. 1 and 2). This involves a tight regulation of synaptic vesicle exocytosis by $\mathrm{Ca}^{2+}$ channels (3-7), which are of Cav1.3 type (8). The $\mathrm{Ca}^{2+}$ channels cluster at the multiple active zones of hair cells $(5$, 9-14). Imaging of $\mathrm{Ca}^{2+}$ indicator fluorescence has revealed localized microdomains of elevated $\left[\mathrm{Ca}^{2+}\right]$ in lower vertebrate hair cells $(10-12,14)$, whereas a spatially less confined rise of submembrane $\left[\mathrm{Ca}^{2+}\right]$ involving $\mathrm{Ca}^{2+}$-induced $\mathrm{Ca}^{2+}$ release (CICR) has been reported for immature mouse inner hair cells (IHCs) (15).

Our understanding of sound encoding in the mammalian cochlea is partly limited by a lack of quantitative information on synaptic $\mathrm{Ca}^{2+}$ signaling in the IHCs of hearing animals. For example, it is believed that differences between the synapses of an individual IHC account for the variability of spontaneous and evoked rates, sound threshold, and dynamic range among spiral ganglion neurons (SGNs) of similar characteristic frequency (16). Presynaptic and postsynaptic mechanisms have been suggested to cause this heterogeneity of SGN dynamics. Differential efferent control of SGN activity (17) seems conceptually obvious. There are also indications for differences in structure (18) and function (19) among active zones of an IHC; however, little is known about the underlying mechanism. Here, we used time-resolved confocal imaging of the fluorescence of low-affinity $\mathrm{Ca}^{2+}$ indicators together with pharmacological manipulations and modeling to characterize synaptic $\mathrm{Ca}^{2+}$ microdomains in IHCs of hearing mice.

\section{Results}

Fast and Localized $\mathrm{Ca}^{2+}$ Signals Mediated by $\mathrm{Ca}^{2+}$ Influx at IHC Active Zones. Voltage activation of $\mathrm{Ca}^{2+}$ influx caused the appearance of submicrometer fluorescence hotspots in the basolateral compartment of IHCs $\left(\left[\mathrm{Ca}^{2+}\right]_{\mathrm{e}}=5 \mathrm{mM}\right.$; Fig. $\left.1 A\right)$ that had been filled with the low-affinity $\mathrm{Ca}^{2+}$ indicator Fluo-5N $\left(400 \mu \mathrm{M}, K_{\mathrm{d}}=95\right.$ $\mu \mathrm{M}$; M. Alp and W. M. Roberts, personal communication) and the slow $\mathrm{Ca}^{2+}$ chelator EGTA $\left(2 \mathrm{mM}, K_{\mathrm{d}}=180 \mathrm{nM}\right.$ at $\left.\mathrm{pH} 7.2\right)$ (20). These conditions ("standard conditions") favored detection of localized $\mathrm{Ca}^{2+}$ signals by augmenting $\mathrm{Ca}^{2+}$ influx (elevated $\left[\mathrm{Ca}^{2+}\right]_{\mathrm{e}}$ ) and limiting intracellular $\mathrm{Ca}^{2+}$ spread (EGTA, e.g., ref. 14). The low affinity of Fluo-5N led us to primarily display fluorescence changes $\left(F-F_{0}\right.$ or $\left.\Delta F\right)$ without background normalization (avoiding an increase in noise due to division by low $F_{0}$ ). In contrast to reports regarding other ribbon synapses (10), we did not observe obvious spot-like $\mathrm{Ca}^{2+}$ indicator fluorescence at rest, arguing against a notable association of the indicator with ribbons. The $\mathrm{Ca}^{2+}$ indicator fluorescence hotspots (spanning hundreds of nanometers) are further on referred to as $\mathrm{Ca}^{2+}$ microdomains to distinguish them from $\mathrm{Ca}^{2+}$ nanodomains, which are implied for $\mathrm{Ca}^{2+}$ signals that operate within a few tens of nanometers of the source and have escaped visualization because of the limited spatial resolution of conventional light microscopes (21).

The $\mathrm{Ca}^{2+}$ microdomains colocalized with synaptic active zones, shown by marking the synaptic ribbons with a rhodamineconjugated CtBP2/RIBEYE-binding peptide (Fig. 1B) (22). We did not observe CtBP2/RIBEYE-marked spots without $\mathrm{Ca}^{2+}$ microdomains. We searched for $\mathrm{Ca}^{2+}$ microdomains in stacks of confocal sections from the base to the apex of the IHC that were acquired during repetitive depolarization. The number of $\mathrm{Ca}^{2+}$ microdomains ( $8 \pm 2$ per IHC, $n=4$ IHCs) was consistent with typical observations of labeled ribbons (e.g., Figs. $1 B$ Left and $5 B)$. Neither $\mathrm{Ca}^{2+}$ microdomains nor ribbons were observed apical to the nucleus. $\mathrm{Ca}^{2+}$ microdomains could not be elicited in the absence of extracellular $\mathrm{Ca}^{2+}$ (Fig. $1 C$, representative for $n=3$ IHCs).

The spatiotemporal properties and voltage dependence of synaptic $\mathrm{Ca}^{2+}$ microdomains were studied at high temporal resolution by using "spot detection" (23) and line scanning. For spot detection, we positioned the laser beam on the brightest pixel in a $\mathrm{Ca}^{2+}$ microdomain of the previously acquired $x y$-scan (white spot in the center of the $\mathrm{Ca}^{2+}$ microdomain of Fig. $2 A$ ) and recorded the fluorescence at an effective rate of $\approx 2 \mathrm{kHz}$ (Methods). Note that we visually chose the best focal plane, routinely performed spot detection at 7 locations in a line (standard method; white spots in Fig. $2 A$, enabling isochronal

Author contributions: T.F. and T.M. designed research; T.F. and D.K. performed research; T.F. and A.N. analyzed data; and T.F., A.N., and T.M. wrote the paper.

The authors declare no conflict of interest.

${ }^{1}$ To whom correspondence should be addressed. E-mail: tmoser@gwdg.de.

This article contains supporting information online at www.pnas.org/cgi/content/full/ 0813213106/DCSupplemental.

๑) 2009 by The National Academy of Sciences of the USA 


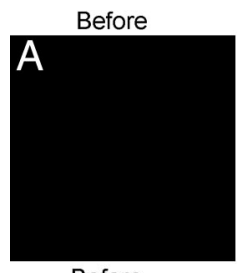

Before

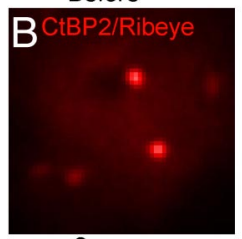

$\left[\mathrm{Ca}^{2+}\right]_{\mathrm{e}} 5 \mathrm{mM}$

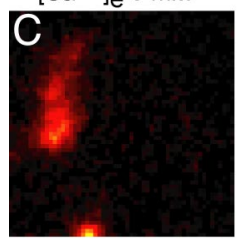

Fig. 1. $\mathrm{Ca}^{2+}$ microdomains mediated by $\mathrm{Ca}^{2+}$ influx at ribbon synapses in IHCs. Confocal images of Fluo-5N-filled IHCs were acquired in 3 repetitions of 6 images at $\approx 10 \mathrm{~Hz}$ : 2 images each before, during, and after stimulation by 200-ms depolarizations to $-7 \mathrm{mV}$. Images averaged over runs and time frame (e.g., before stimulation) are shown. (A) Representative series of images under standard conditions showing 6 Fluo-5N fluorescence hotspots $\left(\mathrm{Ca}^{2+}\right.$ microdomains) during the stimulus. The images were baseline subtracted (subtracting the average of the 2 images before depolarization; $\Delta F$ ). On display are the image during stimulation and those preceding or following the stimulus. $(B) \mathrm{Ca}^{2+}$ microdomains (Center) evolved at ribbon synapses marked by rhodamineconjugated CtBP2/RIBEYE-binding peptide (40 $\mu \mathrm{M}$; Left, acquired before stimulation). Overlay (Right) depicts colocalization of $\mathrm{Ca}^{2+}$ microdomains and synaptic ribbons (both fluorescence channels acquired simultaneously). Note the extension of $\mathrm{Ca}^{2+}$ microdomains beyond ribbons. For 2-dye imaging, acquisition was repeated 6 instead of 3 times. (C) $\mathrm{Ca}^{2+}$ microdomains were abolished by omission of extracellular $\mathrm{Ca}^{2+}\left(\mathrm{Center}\right.$, bath perfusion of nominally $\mathrm{Ca}^{2+}$-free solution and addition of $1 \mathrm{mM}$ EGTA and $5 \mathrm{mM} \mathrm{MgCl}_{2}$ ) and reappeared after readdition of $5 \mathrm{mM}\left[\mathrm{Ca}^{2+}\right]_{\mathrm{e}}$ to the IHC (imaging as in A). (Scale bars: $2 \mu \mathrm{m}$.)

analysis: Fig. 2C) and chose the position with the largest $\Delta F$ for further analysis.

Fig. $2 B-D$ illustrates the rapid build-up and collapse of Fluo-5N fluorescence upon stimulus onset and cessation. We note that the presented kinetics of $\Delta F$ underestimate the true speed of the $\left[\mathrm{Ca}^{2+}\right]$ change because of the limiting binding kinetics of the $\mathrm{Ca}^{2+}$ indicator as well as spatial averaging in the process of detection [supporting information (SI) Fig. S1d and SI Text] (23). The kinetics were quantified by single- or doubleexponential fitting (Fig. $2 B$ and Table S1). Moving the excitation detection volume outside the center of a given $\mathrm{Ca}^{2+}$ microdomain revealed a reduction of $\Delta F$ and progressive slowing of onset and offset kinetics (Fig. $2 B$ and $C$ ). Note the pronounced amplitude variability of $\Delta F$ (Fig. $2 D)$. The distribution of maximal $\Delta F$ values [ $\Delta F_{\max }$, peak fluorescence identified after boxcar (2-ms box) smoothing] had a coefficient of variation (CV) of 0.74 (45 $\mathrm{Ca}^{2+}$ microdomains in $17 \mathrm{IHCs}$ ). Similar variance was observed in the background-normalized data $\left(\Delta F / F_{0}, \mathrm{CV}=0.65\right.$; Fig. S2a) and for the $\Delta F_{\max }$ distribution estimated by fitting Gaussian functions to time-averaged line scans $(\mathrm{CV}=0.75,35$ $\mathrm{Ca}^{2+}$ microdomains in 17 IHCs). We observed a trend toward smaller variance with more precise colocalization of beam position and $\mathrm{Ca}^{2+}$ microdomain center (SI Text), probably reflecting the contribution of measurement variance. Therefore, we consider the most accurate approach (3D spot displacement) to yield the most reliable estimate of $\Delta F_{\max }$ variability $(\mathrm{CV}=$
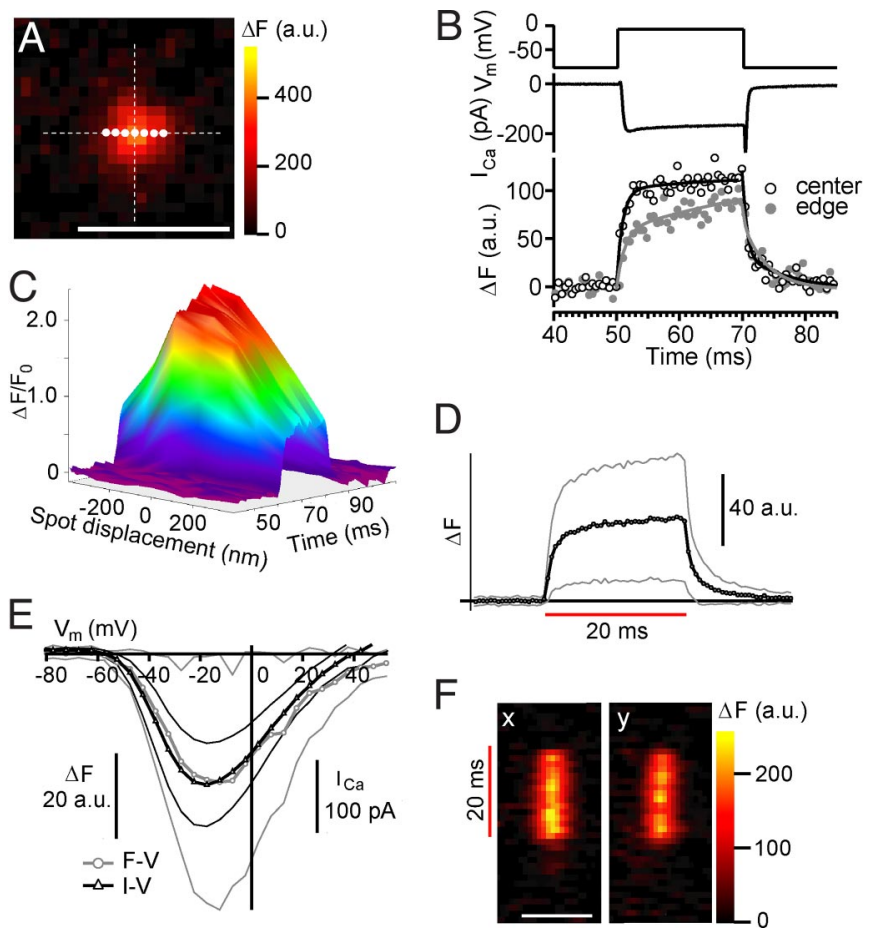

Fig. 2. Spatiotemporal properties and voltage dependence of $\mathrm{Ca}^{2+}$ microdomains. $(A)$ Confocal image of a $\mathrm{Ca}^{2+}$ microdomain illustrating read-out sites for spot detection (white spots) and line scans (orthogonal dashed lines). (Scale bar: $2 \mu \mathrm{m}$.) (B) Representative spot detection experiment: the laser spot was first placed on the brightest pixel in the $x y$ confocal image $(A$, center white spot). Top of the graph shows voltage protocol, middle shows $\mathrm{Ca}^{2+}$ current, and bottom shows $\Delta F$ at the center spot (black) and an outlying position ( 260 $\mathrm{nm}$ off center, gray). $\mathrm{Ca}^{2+}$ current $\left(I_{\mathrm{Ca}}\right)$ and $\Delta F$ represent averages obtained from 5 subsequent runs (interval: $2.25 \mathrm{~s}$ ). Lines represent exponential fits to the $\Delta F$ rise and decay. $(C)$ Isochronal analysis: the laser spot was subsequently displaced bilaterally from the center, and the fluorescence was recorded as described in $B . \Delta F$ traces were assembled in a pseudo-3D plot as a function of time and space. $(D)$ Mean (black) and SD (gray) of $45 \mathrm{Ca}^{2+}$ microdomains (in 17 IHCs) recorded as described in $B$; note the large amplitude variability. For each $\mathrm{Ca}^{2+}$ microdomain, only the maximum intensity recording was considered. $(E)$ Mean and SD of $\Delta F$ (gray) as a function of depolarizing potential $\left(V_{\mathrm{m}}\right)$, obtained from spot-detection experiments at the center of the $\mathrm{Ca}^{2+}$ microdomain ( $n=32 \mathrm{Ca}^{2+}$ microdomains in $17 \mathrm{IHCs}$ ); $\Delta F$ was averaged over the last 15 ms of a 20 -ms stimulus. $\Delta F$ (mean: gray) and $I_{\text {ca }}$ (mean: black) show a similar voltage dependence (thin lines: corresponding SDs). ( $F$ ) Representative line scans ( $x$ and $y$, corresponding to the $x$ and $y$ scan lines in $A$ ). Red bar indicates time of depolarization to $-7 \mathrm{mV}$. (Scale bar: $2 \mu \mathrm{m}$.)

0.54). However, when using this more extensive search, one trades more accuracy in beam position for more rundown of $\mathrm{Ca}^{2+}$ influx and risk of photodamage. Hence, we did not generally apply 3D spot displacement, but used the standard method for most experiments (unless stated otherwise).

Variability of $\Delta F_{\max }$ was only slightly smaller within individual IHCs (Fig. S2 $b-d$ and SI Text), indicating that intracellular variability dominates the variance of the entire population of $\mathrm{Ca}^{2+}$ microdomains. Thus, we interpret the population estimates to indicate intracellular variability throughout. Based on our in situ $F_{\max } / F_{\min }$ ratio $(\approx 50)$ and noting the limitations inherent to $\left[\mathrm{Ca}^{2+}\right]$ quantification using nonratiometric indicators, we estimated the average $\left[\mathrm{Ca}^{2+}\right]$ within the $\mathrm{Ca}^{2+}$ microdomain to be $\approx 3$ $\mu \mathrm{M}$ at the end of a $20-\mathrm{ms}$ depolarization (standard conditions). The voltage dependence of $\Delta F$ qualitatively mimicked that of the simultaneously acquired $\mathrm{Ca}^{2+}$ current, further indicating that $\Delta F$ reflects synaptic $\mathrm{Ca}^{2+}$ influx (Fig. $2 E$ ). The spatial distribution of the $\mathrm{Ca}^{2+}$ signals was quantified by 2 orthogonal line scans (Fig. $2 F$, lines indicated in Fig. $2 A$ ). Following the $\mathrm{Ca}^{2+}$ microdo- 
A

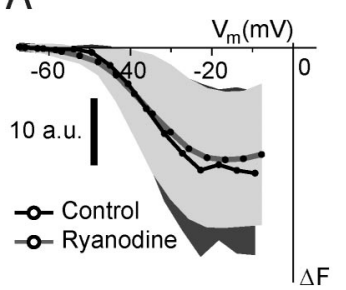

B

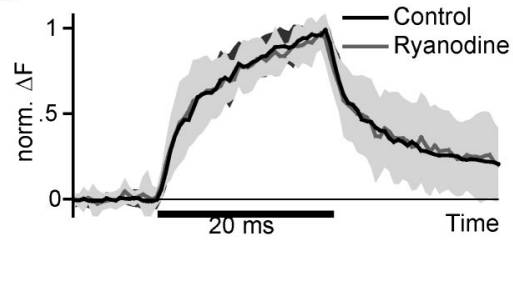

C
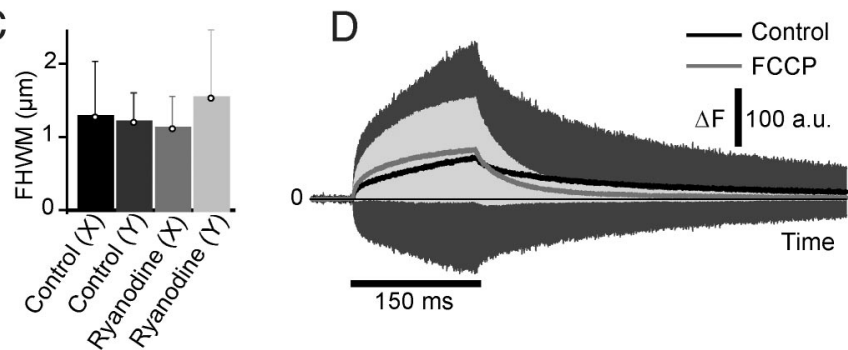

Fig. 3. Blocking $\mathrm{CICR}$ or mitochondrial $\mathrm{Ca}^{2+}$ uptake. $(A)$ Comparable mean (line) and SD (shaded area) of $\Delta F$ vs. membrane voltage $\left(V_{\mathrm{m}}\right)$ for cells loaded with either $100 \mu \mathrm{M}$ ryanodine (12 microdomains in $9 \mathrm{IHCS}$ ) or vehicle (16 microdomains in $13 \mathrm{IHCS}$ ) via the patch pipette ( $\mathrm{KCl}$-based solution with 0.5 mM EGTA; see Methods). $\Delta F$ was obtained by spot detection as described in Fig. $2 E$. For each microdomain, the voltage protocol was repeated 3 times. $V_{\mathrm{m}}$ was offline-corrected for the voltage drop over residual series resistance, which was very relevant here because of the remaining potassium current. $(B)$ Similar onset and decay kinetics with (gray, 15 microdomains in $10 \mathrm{IHCs}$ ) and without (black, 19 microdomains in 14 IHCs) ryanodine. Average (line) and SD (shaded area) of normalized $\Delta F$ traces (spot detection following depolarization to "nominally" $-15.3 \mathrm{mV}$; only recordings with a voltage error $<4 \mathrm{mV}$ were considered). (C) Mean and SD of the FWHM of the $\mathrm{Ca}^{2+}$ microdomain along both perpendicular scan lines (fitting of a Gaussian function to the average of all lines acquired during depolarization). No difference was found between the 2 groups. $X$ (control): $11 \mathrm{Ca}^{2+}$ microdomains in $10 \mathrm{IHCs}, \mathrm{Y}$ (control): $7 \mathrm{Ca}^{2+}$ microdomains in $7 \mathrm{IHCs}, \mathrm{X}$ (ryanodine): $11 \mathrm{Ca}^{2+}$ microdomains in $8 \mathrm{IHCs}$, and $\mathrm{Y}$ (ryanodine): $8 \mathrm{Ca}^{2+}$ microdomains in $6 \mathrm{IHCS}$ ). (D) Mean and SD of $\Delta F$ traces recorded by spot detection in response to 150 -ms depolarizations to $-7 \mathrm{mV}\left(0.5 \mathrm{mM}\right.$ EGTA and $375 \mu \mathrm{M}$ Fluo-4FF in the pipette; $10 \mathrm{mM}\left[\mathrm{Ca}^{2+}\right]_{\mathrm{e}}$ and $5 \mu \mathrm{M}$ BayK8644 in the bath) in control experiments $\left(n=11 \mathrm{Ca}^{2+}\right.$ microdomains in $7 \mathrm{lHCs})$, and in intracellular presence of FCCP $(10 \mu \mathrm{M})$ and oligomycin (2.5 mg/mL), blocking mitochondrial $\mathrm{Ca}^{2+}$ uptake $\left(n=23 \mathrm{Ca}^{2+}\right.$ microdomains in $10 \mathrm{IHCS}$ ).

main build-up during the first 1-2 ms, there was little spatial spread during the course of the depolarization under standard conditions.

Exploring the Mechanisms Underlying the $\mathrm{Ca}^{2+}$ Microdomain Heterogeneity. What differentiates the $\mathrm{Ca}^{2+}$ signals between synapses of a given IHC? Possible mechanisms include disparities in $\mathrm{Ca}^{2+}$ release from intracellular stores (e.g., CICR), $\mathrm{Ca}^{2+}$ buffering, and/or $\mathrm{Ca}^{2+}$ sequestration. Moreover, and most importantly, there could be differences in any of the 3 determinants of $\mathrm{Ca}^{2+}$ influx: number of channels, open probability, and single-channel current.

CICR mediated by ryanodine receptors could amplify a $\mathrm{Ca}^{2+}$ influx-mediated $\left[\mathrm{Ca}^{2+}\right]$ rise to various degrees. We tested for a potential contribution of CICR to presynaptic $\mathrm{Ca}^{2+}$ signals by comparing the spatiotemporal properties and the voltage dependence of the $\mathrm{Ca}^{2+}$ microdomains elicited by $20-\mathrm{ms}$ depolarizations in the presence or absence of a ryanodine receptor antagonist (100 $\mu \mathrm{M}$ ryanodine in the pipette, Fig. $3 A-C ; 20$ or $40 \mu \mathrm{M}$ ryanodine in the bath, Fig. S3). To favor the detection of a small CICR contribution, we entailed weaker cytosolic $\mathrm{Ca}^{2+}$ buffering ( $0.5 \mathrm{mM}$ EGTA). None of these conditions caused a significant change in $\Delta F$ (Fig. $3 A$ and Table $\mathrm{S} 1$ ), the rise and decay kinetics (Fig. 3B), spatial distribution (Fig. 3C), the voltage of half-maximal $\Delta F$ activation, or the variability of $\Delta F_{\max }$ (Tables $\mathrm{S} 1$ and S2). These results are consistent with observing comparable amplitudes and amplitude scatter, regardless of whether $\mathrm{K}^{+}$or $\mathrm{Cs}^{+}$[which blocks CICR (15)] was used as the main intracellular cation. In summary, we conclude that CICR does not contribute significantly to synaptic $\mathrm{Ca}^{2+}$ signaling in mature IHCs and cannot explain the observed synaptic heterogeneity.

Synaptic $\mathrm{Ca}^{2+}$ removal may differ across IHC active zones. $\mathrm{Ca}^{2+}$ may be cleared temporarily by mitochondria (24) or bind to immobile cytosolic $\mathrm{Ca}^{2+}$ binding sites $(25,26)$. For sensitive detection of $\mathrm{Ca}^{2+}$ changes due to mitochondrial $\mathrm{Ca}^{2+}$ uptake, we used a $\mathrm{Ca}^{2+}$ indicator with higher affinity (Fluo-4FF, $375 \mu \mathrm{M}$, $K_{\mathrm{d}}=10 \mu \mathrm{M}$; M. Alp and W. M. Roberts, personal communication) and weak cytosolic $\mathrm{Ca}^{2+}$ buffering (0.5 mM EGTA). Under these conditions, blocking mitochondrial $\mathrm{Ca}^{2+}$ uptake by intracellular carbonyl cyanide 4-(trifluoromethoxy)phenylhydrazone (FCCP, $10 \mu \mathrm{M}$, coapplied with oligomycin, $2.5 \mathrm{mg} / \mathrm{mL}$ ) did not alter the $\mathrm{Ca}^{2+}$ microdomains, except for making the slow component of the fluorescence rise slightly faster (Fig. $3 D$ and Table S1). Moreover, the CVs of the $\mathrm{Ca}^{2+}$ microdomain amplitudes were in the same range (1.06 vs. 0.91 for control and FCCP, respectively), arguing against a prominent contribution of variable mitochondrial $\mathrm{Ca}^{2+}$ uptake to heterogeneity of synaptic $\mathrm{Ca}^{2+}$ signals.

Addition of EGTA $(0.5,2$, and $10 \mathrm{mM})$ to the pipette solution was used to study the effects of mobile $\mathrm{Ca}^{2+}$ buffers on the spatiotemporal properties of $\mathrm{Ca}^{2+}$ microdomains and their heterogeneity in $5 \mathrm{mM}\left[\mathrm{Ca}^{2+}\right]_{\mathrm{e}}$. For comparison, we performed experiments under close to physiological conditions ("native" $\mathrm{Ca}^{2+}$ buffering, perforated-patch configuration, physiological $\left.\left[\mathrm{Ca}^{2+}\right]_{\mathrm{e}}: 1.3 \mathrm{mM}\right)$. For dye loading, the perforated-patch recordings were preceded by a brief whole-cell episode $(15-30 \mathrm{~s}, 2 \mathrm{mM}$ [Fluo-5N] pipette), avoiding major loss of cellular $\mathrm{Ca}^{2+}$-buffering proteins (SI Text).

Spots were identified in each condition, but were of smaller amplitude and spatially more confined with higher $\mathrm{Ca}^{2+}$ buffering capacity (Fig. $4 A-C$ and $E$ ). The rise and decay kinetics as well as the spatial distribution of $\mathrm{Ca}^{2+}$ microdomains observed in perforated-patch experiments were most similar to those found with $0.5 \mathrm{mM}$ EGTA. To quantitatively characterize activezone $\mathrm{Ca}^{2+}$ dynamics in the well-defined whole-cell experiments, we compared the experimental data to predictions of a model of $\mathrm{Ca}^{2+}$ influx, diffusion, and binding by using CalC software (27) and simulating $\mathrm{Ca}^{2+}$ indicator fluorescence detection (for details, see Fig. S1, SI Text, and Table S3). Mobile endogenous $\mathrm{Ca}^{2+}$ binding sites, which are progressively "washed out" into the pipette in the whole-cell configuration, were disregarded, as were mitochondrial $\mathrm{Ca}^{2+}$ uptake and CICR. $\Delta F$ was predicted by transforming the spatiotemporal distribution of $\mathrm{Ca}^{2+}$-bound Fluo-5N by our in situ $F_{\max } / F_{\min }$ ratio $(\approx 50)$, as described (28), and convolution with the point spread function of the optical setup. The model predicted the mean properties of the fluorescent hotspots under the different conditions reasonably well (Fig. $4 C-E$ ).

Experiments and model consistently showed biexponential rise and decay kinetics in weak exogenous buffering conditions, whereas the slow components were strongly diminished by increasing EGTA (Fig. $4 D$ and Table S1). In addition, high EGTA accelerated the fast $\mathrm{Ca}^{2+}$ decay (Fig. $4 D$ and Table S1). The widths of the $\mathrm{Ca}^{2+}$ microdomains in different EGTA concentrations were well predicted by the model (Fig. $4 E$ ). The width of the $\mathrm{Ca}^{2+}$ microdomains increased throughout the depolarization $(20 \mathrm{~ms})$ for lower concentrations, but it rapidly reached steady state with EGTA $2 \mathrm{mM}$ or greater (Fig. S4). The choice of the $\mathrm{Ca}^{2+}$-buffering condition did not appreciably affect the experimentally observed synaptic heterogeneity for any of the quantified parameters (Fig. $4 B$ and Table S1). Finding 

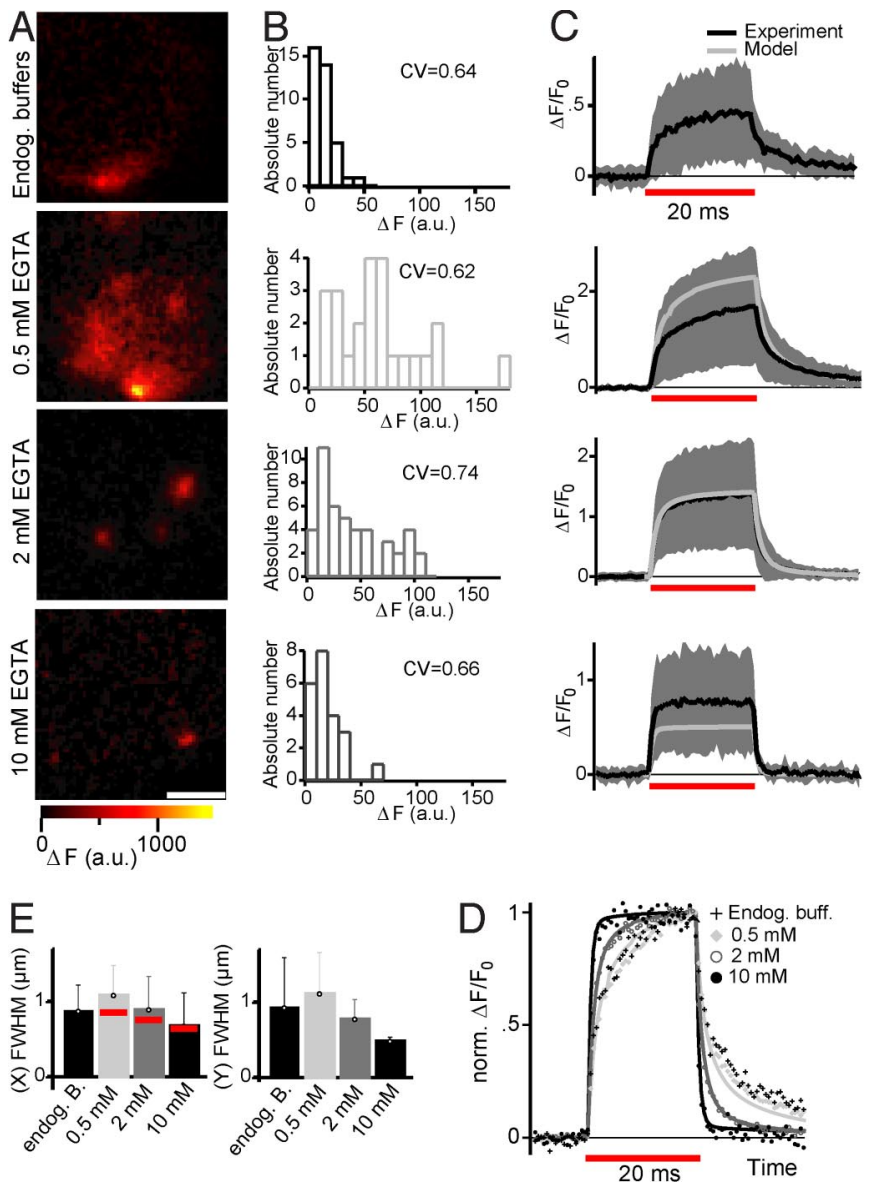

Fig. 4. Effects of $\mathrm{Ca}^{2+}$ buffering on $\mathrm{IHC} \mathrm{Ca}{ }^{2+}$ microdomains. $(A)$ Representative confocal $\Delta F$ images of IHCs (recorded as introduced in Fig. $1 A$ ) with close to native endogenous $\mathrm{Ca}^{2+}$ buffering (perforated-patch, top row, representing $37 \mathrm{Ca}^{2+}$ microdomains in $14 \mathrm{IHCS}$ ) or various amounts of exogenously added EGTA: $0.5 \mathrm{mM}$ (second row, representing $24 \mathrm{Ca}^{2+}$ microdomains in 15 IHCs), $2 \mathrm{mM}$ (third row, representing $45 \mathrm{Ca}^{2+}$ microdomains in $17 \mathrm{IHCs}$ ), or $10 \mathrm{mM}$ (bottom row, representing $22 \mathrm{Ca}^{2+}$ microdomains in $7 \mathrm{IHCs}$ ). (Scale bar: $2 \mu \mathrm{m}$.) (B) Distributions of $\Delta F_{\max }$ obtained for the buffering conditions in spot-detection experiments after box-car smoothing (2-ms box). (C) Experimental mean (black line) and SD (gray area) of $\Delta F / F_{0}$ traces as well as model prediction (gray line) for the respective buffering conditions. Model predictions were obtained only for exogenous buffering experiments (see Table S1 and $S I$ Text) $(D)$ Normalized mean $\Delta F / F_{0}$ traces of all 4 buffering conditions (markers) emphasizing the kinetic differences, and model predictions (lines) for exogenous buffering experiments. $(E)$ Mean and SD of FWHM of the $\mathrm{Ca}^{2+}$ microdomain along both scan lines (fitting of a Gaussian function to the time-averaged line profile). Also shown are the model predictions (red bars).

comparable synaptic heterogeneity with an excess of mobile $\mathrm{Ca}^{2+}$ buffer renders differences in local $\mathrm{Ca}^{2+}$ buffering unlikely to contribute substantially.

Heterogeneity of $\mathrm{Ca}^{2+}$ influx is an attractive candidate mechanism, under direct control of the membrane potential. Indeed, we observed substantial variability in the voltage dependence of $\Delta F$ across the $\mathrm{Ca}^{2+}$ microdomains (Fig. $5 A$ ), even within a given IHC (dotted lines in Fig. $5 A$ ), which probably reflected differences in gating. The variance of the voltage of half-maximal activation was larger for the $\mathrm{Ca}^{2+}$ microdomains $\left(24 \mathrm{mV}^{2}\right.$; standard conditions, see Table S1 for other conditions) than for the $\mathrm{Ca}^{2+}$ current in the corresponding IHCs $\left(5 \mathrm{mV}^{2}\right)$. Voltage errors due to changes in electrode potential were found to be less than $3 \mathrm{mV}$, and series resistance-related errors were corrected offline.

However, substantial $\Delta F$ variability was observed also for
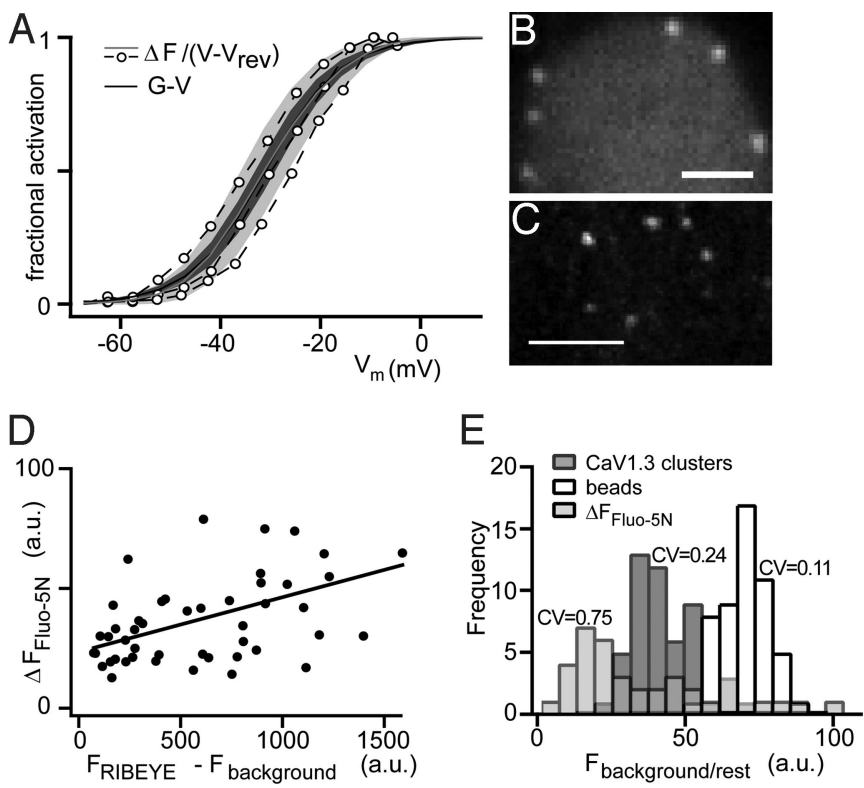

Fig. 5. Heterogeneous voltage dependence and $\mathrm{Ca}^{2+}$ channel number of synaptic $\mathrm{Ca}^{2+}$ channel clusters in IHCs. $(A)$ To demonstrate the variable voltage dependence of $\Delta F_{\max }$, we display the average results (and SD) of fitting a Boltzmann function to the fractional voltage activation of $\Delta F[\Delta F$ divided by the driving force $\left(V-V_{\text {rev }}\right)$ of the underlying $\mathrm{Ca}^{2+}$ current]. Note that at -7 $\mathrm{mV}$, the potential used for comparisons in Figs. $2 D$ and $F, 3 B-D, 4$, and $5 D$ and $E$, the activation of $\Delta F$ (mean: gray line, SD: light gray area) and $I_{\mathrm{Ca}}$ (mean: black line, SD: dark gray area) is nearly complete. Note the pronounced variability in the voltage dependence of activation, even within 1 cell (dashed traces: individual data curves from $3 \mathrm{Ca}^{2+}$ microdomains in an IHC). (B) shows an $x y$ scan through the basal portion of an IHC loaded with rhodamine-conjugated, CtBP2/RIBEYE-binding peptide (40 $\mu \mathrm{M}$; dissolved in intracellular solution). Note the spots of increased fluorescence intensity, indicative of synaptic ribbons. (Scale bar: $2 \mu \mathrm{m}$.) (C) Representative confocal section of an IHC stained with a Cav1.3 antibody. (Scale bar: $2 \mu \mathrm{m}$.) (D) Correlation between $\mathrm{Ca}^{2+}$ microdomain $\Delta F_{\max }$ and fluorescence intensity of the corresponding ribbon (amplitude of 2D Gaussian function, fit to the fluorescently tagged ribbon in $x y$ scans acquired at rest and before spot detection). The microdomain $\Delta F_{\text {max }}$ was obtained at the maximum-intensity location, identified by a series of 11 axially (along optical axis) displaced measurements (200-nm steps). Note the positive correlation $\left(P_{\mathrm{r}}=0.47, P<0.01 ; n=48 F_{\mathrm{Ca}}-F_{\mathrm{RIBEYE}}\right.$ pairs in 18 IHCS). (E) Distributions of $\mathrm{Ca}^{2+}$ microdomain $\Delta F_{\max }$ (amplitude of 1D Gaussian fit to time-averaged line-scan profile, $n=35)$, Cav1.3 immunofluorescence $(n=50)$, and 210-nm crimson bead fluorescence $(n=52)$.

saturating stimuli (at $-7 \mathrm{mV}$ : Fig. $5 A$; see Figs. 2, 4, and 5 for illustration). Next, we tested whether the dihydropyridine L-type channel agonist BayK8644 (29) could reduce the $\Delta F$ heterogeneity of the $\mathrm{Ca}^{2+}$ microdomains. By uniformly increasing the open probability of Cav1.3 channels in IHCs (5), we reasoned that BayK8644 would equalize stimulus-dependent $\mathrm{Ca}^{2+}$ influx between IHC active zones. To prevent local $\mathrm{Ca}^{2+}$ saturation of Fluo-5N, we added a high concentration of EGTA $(10 \mathrm{mM}$ in the pipette, shown above to not reduce heterogeneity of $\Delta F$ ). However, despite the maximized open probability, we still found large variability in $\Delta F\left(\mathrm{CV}=0.87,16 \mathrm{Ca}^{2+}\right.$ microdomains in 5 IHCs) (Table S1).

The $\mathrm{Ca}^{2+}$ channel number per active zone has been suggested to vary with the size of the synaptic ribbon in hair cells in chick, frog, and turtle hair cells (30). This was based on a correlation of the estimates of $\mathrm{Ca}^{2+}$ channel number and of total release area (product of synapse number and ribbon size) per hair cell. Here, we explored this hypothesis at the single-synapse level and asked whether differences in channel number could contribute to the heterogeneity of $\mathrm{Ca}^{2+}$ microdomain amplitudes. We related the $\mathrm{Ca}^{2+}$ microdomain amplitude (Fluo-5N $\Delta F$, spot detection, 
20-ms depolarizations to $-7 \mathrm{mV}$ ) to the fluorescence intensity of the corresponding peptide-labeled ribbons before depolarization (Fig. $1 B$ and $5 B$ ). Ribbon fluorescence intensity (amplitude of 2D Gaussian function fit) rather than FWHM was used for approximation of ribbon size, because ribbons are too small to be faithfully measured by confocal microscopy (31). Relating the fluorescence intensity to ribbon size seems reasonable, as previous work (22) indicated a quantitative scaling of ribbon labeling by the fluorescent $\mathrm{CtBP} 2 / \mathrm{RIBEYE}$-binding peptide with the number of contained RIBEYE molecules. As shown in Fig. $5 D$, we did observe a significant correlation between $\mathrm{Ca}^{2+}$ microdomain amplitude and the ribbon fluorescence intensity. This suggests that larger $\mathrm{Ca}^{2+}$ microdomain amplitudes arose from bigger active zones, probably containing more $\mathrm{Ca}^{2+}$ channels, and that differences in $\mathrm{Ca}^{2+}$ channel number among synapses can account for part of the variance of their $\mathrm{Ca}^{2+}$ microdomain amplitudes.

The attractive hypothesis of a variable $\mathrm{Ca}^{2+}$ channel content was tested by confocal microscopy of active zones following Cav1.3 immunolabeling (Fig. 5C). We estimated the fluorescence intensity as described above for ribbons. Images were acquired using a low numerical aperture $(0.7)$ objective lens to ensure that most of the Cav1.3 immunofluorescent spot was included in the excitation-detection volume. We reasoned that active zones with larger $\mathrm{Ca}^{2+}$ channel clusters or clusters with higher $\mathrm{Ca}^{2+}$ channel densities would display higher fluorescence intensities (assuming a linear labeling reaction). The measurement revealed considerable variance among the synapses $(\mathrm{CV}=0.24$; Fig. $5 E)$. We also measured fluorescent beads using the same microscope and detection settings (diameter $210 \mathrm{~nm}$; Fig. $5 E$ ) to get an upper boundary of the imaging- and analysis-related contributions $(\mathrm{CV}=0.11)$. For comparison, Fig. $5 E$ also displays the fluorescence intensity distribution of Fluo-5N hotspots.

\section{Discussion}

Toward a Quantitative Understanding of the Active-Zone $\mathrm{Ca}^{2+} \mathrm{Mi}-$ crodomain in IHCs. Studying IHCs of mice after the onset of hearing (low-affinity $\mathrm{Ca}^{2+}$ indicator, 0.5-10 mM EGTA), we observed spatially confined $\mathrm{Ca}^{2+}$ domains much like those reported for lower vertebrate hair cells $(10-12,14)$. Based on our experiments, localized domains of high $\mathrm{Ca}^{2+}$ concentration are likely to occur at IHC synapses with physiological $\mathrm{Ca}^{2+}$ influx and native $\mathrm{Ca}^{2+}$ buffering. Several lines of evidence suggest that the observed $\mathrm{Ca}^{2+}$ microdomains arose from $\mathrm{Ca}^{2+}$ influx through synaptic $\mathrm{Ca}_{V} 1.3 \mathrm{Ca}^{2+}$ channel clusters at the ribbon synapses. First, they occurred at sites marked by a ribbonlabeling, CtBP2/RIBEYE-binding, fluorescent peptide, and their number was consistent with counts of ribbon synapses (this study, and ref. 31). This argues against an appreciable fraction of presynaptically silent synapses as well as against $\mathrm{Ca}^{2+}$ microdomains occurring outside ribbon-type active zones. Second, $\mathrm{Ca}^{2+}$ microdomains were not observed when removing extracellular $\mathrm{Ca}^{2+}$. Third, their amplitude shared the voltage dependence of the whole-cell $\mathrm{Ca}^{2+}$ current. Fourth, inhibition of CICR by ryanodine or intracellular $\mathrm{Cs}^{+}$did not reduce the amplitude of the $\mathrm{Ca}^{2+}$ microdomains. Finally, the fluorescence changes could be reasonably well predicted by realistic modeling of active-zone $\mathrm{Ca}^{2+}$ signals, driven solely by $\mathrm{Ca}^{2+}$ influx, without invoking additional $\mathrm{Ca}^{2+}$ sources.

Experiments with different EGTA concentrations and modeling were used to explore how mobile $\mathrm{Ca}^{2+}$ buffering shapes the spatiotemporal properties of the $\mathrm{Ca}^{2+}$ microdomains. In brief, the more mobile $\mathrm{Ca}^{2+}$ binding sites were present, the faster, smaller, and spatially more constrained were the observed and simulated $\mathrm{Ca}^{2+}$ changes. This can be intuitively understood to reflect a faster $\mathrm{Ca}^{2+}$ capturing and less $\mathrm{Ca}^{2+}$ buffer saturation. Mobile $\mathrm{Ca}^{2+}$ buffering presented the prevailing $\mathrm{Ca}^{2+}$ removal mechanism, limiting the active zone's $\mathrm{Ca}^{2+}$ signal of IHCs in space and time during short depolarizations. This agrees well with conclusions of previous studies on other hair cells $(11,12)$.

Heterogeneity of Synaptic $\mathrm{Ca}^{2+}$ Signals: Mechanisms and Consequences. The voltage dependence varies even among active zones of an individual IHC, which may relate to the observation of different stimulus thresholds for styryl dye destaining across putative ribbon synapses (19). Because the hair cell is isopotential (9), voltage errors due to series resistance were corrected, and those due to drifts in electrode potential were minor, we expect experimental procedures to contribute little to the observed variance of the voltage of half activation $\left(24 \mathrm{mV}^{2}\right)$, which was 4 times larger than that of the whole-cell current compared among the same experiments.

Besides shifts in activation curves, we also found a large $\Delta F_{\max }$ variability of $\mathrm{Ca}^{2+}$ microdomains for strong depolarizations $(-7$ $\mathrm{mV}$ ) when $\mathrm{Ca}^{2+}$ channel activation saturates and even after maximization of channel open probability by BayK8644. Although some contribution of the measurement to the observed variance of $\mathrm{Ca}^{2+}$ microdomain amplitude is likely, this indicates variability of $\mathrm{Ca}^{2+}$ signaling in addition to that arising from differences in voltage gating (discussed above). Our data do not support a major contribution of $\mathrm{Ca}^{2+}$ signal amplification by CICR, local differences in $\mathrm{Ca}^{2+}$ buffering/sequestration, or the average maximal $\mathrm{Ca}^{2+}$ channel open probability among IHC synapses.

Heterogeneity in the number of $\mathrm{Ca}^{2+}$ channels among hair cell active zones could explain a substantial part of the observed amplitude variability of $\mathrm{Ca}^{2+}$ microdomains at saturating depolarizations. Confocal microscopy of immunolabeled Cav1.3 channel clusters indicates that the number of $\mathrm{Ca}^{2+}$ channels indeed varies among hair cell synapses $(\mathrm{CV}=0.24$; Fig. 5). This result was obtained from measurements on fixed tissue, and therefore not from those synapses that had been functionally characterized. However, the argument is supported by the simultaneous imaging of $\mathrm{Ca}^{2+}$ microdomains and their corresponding, fluorescently labeled ribbons. The observed positive correlation of both fluorescence intensities suggests that larger $\mathrm{Ca}^{2+}$ microdomains arise from active zones with more $\mathrm{Ca}^{2+}$ channels. This hypothesis assumes that $(i)$ the fluorescence of the peptide-labeled ribbon indicates the number of RIBEYE molecules (22) and (ii) can be used to approximate ribbon size, (iii) which scales with the spatial extent of the active zone and the number of $\mathrm{Ca}^{2+}$ channels (30). Modeling confirmed that under our experimental conditions, the contributions of individual $\mathrm{Ca}^{2+}$ channels at the active zone sum roughly linearly to set the amplitude of the $\mathrm{Ca}^{2+}$ microdomain reported by the low-affinity indicator Fluo-5N and our imaging system (Fig. S1e and SI Text). Whether the remaining mismatch of amplitude variance between $\mathrm{Ca}^{2+}$ microdomain and $\mathrm{Ca}_{V} 1.3$ channel immunofluorescence reflects differences in $\mathrm{Ca}^{2+}$ channel regulation among synapses remains to be addressed in future studies.

In summary, we propose that the IHC adjusts both the number of $\mathrm{Ca}^{2+}$ channels and their gating at its individual synapses. This could explain the coexistence of synapses within an individual hair cell that drive different SGN firing rates. Transmitter release from IHCs seems to be controlled by $\mathrm{Ca}^{2+}$ nanodomains around $\mathrm{Ca}^{2+}$ channels within few tens of nanometers of the docked vesicle $(2,5$, $7,32)$. In this case, the number of open channels would relate linearly to the rate of vesicle exocytosis. This concept has been primarily tested for fusion of the readily releasable pool of vesicles $(5,7)$, but probably also applies during longer stimuli $(6)$. Differences in the number of channels opening for given receptor potential amplitude would translate into different peak and adapted firing rates. However, synapses with more $\mathrm{Ca}^{2+}$ channels and/or a more negative voltage range of channel activation would also cause higher spontaneous firing rates, which in SGNs seem entirely driven by hair cell transmitter release (33). The question of how these 
properties are differentially regulated in a single IHC remains to be addressed in future studies.

\section{Methods}

Animals. C57BL/6 mice (ages 14-18 days) were used for experiments.

Patch-Clamp and Confocal $\mathrm{Ca}^{2+}$ Imaging. IHCs from apical coils of freshly dissected organs of Corti were patch-clamped as described previously (4). The pipette solution contained (in mM): 130 cesium glutamate, 13 tetraethylammonium (TEA)-Cl, $20 \mathrm{CsOH}-\mathrm{Hepes}$, and $1 \mathrm{MgCl}_{2}$, and $250 \mu \mathrm{g} / \mathrm{mL}$ amphotericin $\mathrm{B}$ ( $\mathrm{pH}$ 7.2) for perforated-patch recordings, in addition to (in mM) 2 MgATP, 0.3 $\mathrm{NaGTP}$, and various concentrations of EGTA (standard: $2 \mathrm{mM}$; cesium glutamate concentration adjusted for $10 \mathrm{mM}$ EGTA), $0.4 \mathrm{mM}$ Fluo- $5 \mathrm{~N}$ or $0.375 \mathrm{mM}$ Fluo-4FF (penta- $\mathrm{K}^{+}$salts; Invitrogen) $(\mathrm{pH} 7.0)$ for standard whole-cell recordings, and for CICR experiments (in mM): $155 \mathrm{KCl}, 20 \mathrm{KOH}-\mathrm{Hepes}, 2 \mathrm{MgATP}$, and $0.3 \mathrm{NaGTP}$ ( $\mathrm{pH}$ 7.0). The extracellular solution contained (in $\mathrm{mM}$; if deviating, concentrations used for CICR experiments are given in brackets): 102 [133] $\mathrm{NaCl}, 35$ [3] TEA-Cl, 2.8 [5.8] KCl, $5 \mathrm{CaCl}_{2}$ (1.3 for perforated-patch recordings; balanced by $\mathrm{NaCl}), 1 \mathrm{MgCl}_{2}, 10 \mathrm{NaOH}$-Hepes, and $10 \mathrm{D}$-glucose ( $\left.\mathrm{pH} 7.3\right)$. BayK8644 (5 $\mu \mathrm{M}$; Tocris) was bath applied. Ryanodine (Calbiochem) was either dissolved in the pipette solution $(100 \mu \mathrm{M})$ or continuously bath applied (20 or $40 \mu \mathrm{M})$. FCCP (10 $\mu \mathrm{M}$; Fluka) and oligomycin ( $2.5 \mathrm{mg} / \mathrm{mL}$; Sigma) were intracellularly applied. An EPC-9 amplifier and Patchmaster software (HEKA Elektronik) were used for measurements. All voltages were corrected for liquid-junction potentials ( $17.5 \mathrm{mV}$ for cesium glutamate solution and $5.3 \mathrm{mV}$ for $\mathrm{KCl}$ solution) and voltage drops across series resistance. Currents were low-pass-filtered at $5 \mathrm{kHz}$ and sampled at $50 \mathrm{kHz}$. Cells with a membrane current exceeding $-50 \mathrm{pA}$ at our standard holding potential of $-87.5 \mathrm{mV}$ (cesium glutamate; for $\mathrm{KCl}$ solution, $-75.3 \mathrm{mV}$ ) were discarded from analysis. $\mathrm{Ca}^{2+}$ currents were further isolated from background current by using a P/n protocol. Current-voltage relationships were fitted by using a Boltzmann function. Interstimulus periods were $2-3 \mathrm{~s}$ between sweeps and 1-2 min between ensembles. The average $\mathrm{Ca}^{2+}$ current rundown at the end of the experiment was $30 \%$ of the maximum current.

$\mathrm{Ca}^{2+}$ imaging was performed with a Fluoview 300 confocal scanner mounted on an upright microscope (BX50WI) equipped with a 0.9 numerical aperture, 60X water-immersion objective (all from Olympus). A 50-mW, 488-nm laser (Cyan; Newport Spectraphysics) was used for excitation of Fluo$5 \mathrm{~N}$, and a 1.5-mW, 543-nm He-Ne laser was used for excitation of rhodamineconjugated, CtBP2/RIBEYE-binding peptide. $\mathrm{Ca}^{2+}$ microdomains were identi-

1. Fuchs PA, Glowatzki E, Moser T (2003) The afferent synapse of cochlear hair cells. Curr Opin Neurobiol 13:452-458.

2. Moser T, Neef A, Khimich $D$ (2006) Mechanisms underlying the temporal precision of sound coding at the inner hair cell ribbon synapse. J Physiol 576:55-62.

3. Parsons TD, Lenzi D, Almers W, Roberts WM (1994) Calcium-triggered exocytosis and endocytosis in an isolated presynaptic cell: Capacitance measurements in saccular hair cells. Neuron 13:875-883.

4. Moser T, Beutner D (2000) Kinetics of exocytosis and endocytosis at the cochlear inner hair cell afferent synapse of the mouse. Proc Natl Acad Sci USA 97:883-888.

5. Brandt A, Khimich D, Moser T (2005) Few CaV1.3 channels regulate the exocytosis of a synaptic vesicle at the hair cell ribbon synapse. J Neurosci 25:11577-11585.

6. Keen EC, Hudspeth AJ (2006) Transfer characteristics of the hair cell's afferent synapse. Proc Natl Acad Sci USA 103:5537-5542.

7. Goutman JD, Glowatzki E (2007) Time course and calcium dependence of transmitter release at a single ribbon synapse. Proc Natl Acad Sci USA 104:16341-16346.

8. Platzer J, et al. (2000) Congenital deafness and sinoatrial node dysfunction in mice lacking class D L-type $\mathrm{Ca}^{2+}$ channels. Cell 102:89-97.

9. Roberts WM, Jacobs RA, Hudspeth AJ (1990) Colocalization of ion channels involved in frequency selectivity and synaptic transmission at presynaptic active zones of hair cells. J Neurosci 10:3664-3684.

10. Issa NP, Hudspeth AJ (1994) Clustering of $\mathrm{Ca}^{2+}$ channels and $\mathrm{Ca}^{2+}$-activated $\mathrm{K}^{+}$channels at fluorescently labeled presynaptic active zones of hair cells. Proc Natl Acad Sci USA 91:7578-7582.

11. Tucker T, Fettiplace R (1995) Confocal imaging of calcium microdomains and calcium extrusion in turtle hair cells. Neuron 15:1323-1335.

12. Issa NP, Hudspeth AJ (1996) The entry and clearance of $\mathrm{Ca}^{2+}$ at individual presynaptic active zones of hair cells from the bullfrog's sacculus. Proc Natl Acad Sci USA 93:9527-9532.

13. Rodriguez-Contreras A, Yamoah EN (2001) Direct measurement of single-channel $\mathrm{Ca}^{2+}$ currents in bullfrog hair cells reveals two distinct channel subtypes. J Physio/ 534:669-689.

14. Zenisek D, Davila V, Wan L, Almers W (2003) Imaging calcium entry sites and ribbon structures in two presynaptic cells. J Neurosci 23:2538-2548.

15. Kennedy HJ, Meech RW (2002) Fast $\mathrm{Ca}^{2+}$ signals at the mouse inner hair cell synapse: A role for $\mathrm{Ca}^{2+}$ induced $\mathrm{Ca}^{2+}$ release. J Physiol 539:15-23.

16. Liberman MC (1980) Morphological differences among radial afferent fibers in the cat cochlea: An electron-microscopic study of serial sections. Hear Res 3:45-63.

17. Ruel J, et al. (2001) Dopamine inhibition of auditory nerve activity in the adult mammalian cochlea. Eur J Neurosci 14:977-986. fied in xy scans during 200-ms depolarizations $[0.5 \%$ of maximum laser intensity $(488 \mathrm{~nm})]$ and were further characterized by using spot detection and line scans. During spot detection $[0.05 \%$ of maximum laser intensity (488 $\mathrm{nm})]$, the output of the photomultiplier tube (PMT) signal $(500 \mathrm{kHz}$ ) was temporally averaged to yield an effective sampling rate of $1.85 \mathrm{kHz}$. Line scans were acquired at a rate of $0.74 \mathrm{kHz}[0.25 \%$ of max laser intensity $(488 \mathrm{~nm})]$. Isochronal spot detection measurements and line scans were repeated 5 and 10 times, respectively. For 2-dye imaging, the laser intensities ( $488 \mathrm{~nm}$ ) were doubled. The 543-nm laser was operated at the following intensities: $50 \%$ for $x y$ scans, $25 \%$ for line scans, and $10 \%$ for spot detection. PMT dark current was subtracted for all measurements, and PMT settings were identical for all experiments. In rare cases, we observed an unclear long-term rise in fluorescence that was independent of leak current amplitude.

Immunohistochemistry. The freshly dissected apical cochlear turns were fixed for $25 \mathrm{~min}$ in $99 \%$ methanol at $-20^{\circ} \mathrm{C}$. Immunostaining was performed as described in ref. 31. The following antibodies were used: mouse IgG1 antiCtBP2 (also recognizing the ribbon protein RIBEYE; 1:150; BD Biosciences), rabbit-anti-Cav1.3 (1:75; Alomone Labs), and secondary AlexaFluor488labeled and AlexaFluor568-labeled antibodies (1:200; Molecular Probes). Confocal images of Cav1.3-immunolabeled IHCs were acquired by using an SP5 confocal microscope (Leica) with 488-nm (Ar) and 561-nm (DPSS) lasers for excitation and a $63 \times$ oil immersion objective (numerical aperture 0.7 ).

Model and Data Analysis. Fig. S1, SI Text, and Table S3 provide a detailed description of the model using CalC (http://web.njit.edu/ matveev/calc.html). Data are presented as mean and SD. Igor Pro 6 (Wavemetrics) was used for analysis.

ACKNOWLEDGMENTS. We thank A. Egner for contribution to the Cav1.3 immunofluorescence imaging; V. Matveev for providing CalC simulation software; D. Zenisek and G. Matthews for providing fluorescently labeled CtBP2/RIBEYE-binding peptide; F. Wolf and E. Neher for discussion; and E. Neher F. Wolf, A. Lee, N.M. Chapochnikov, and T. Sakaba for their comments on the manuscript. This work was supported by a Lichtenberg Fellowship of the state of Lower Saxony (to T.F.) and grants from the Deutsche Forschungsgemeinschaft, Center for Molecular Physiology of the Brain, the Human Frontier Science Program (to T.M.), the European Commission (EuroHear; to T.M.), the Max Planck Society (tandem project, to T.M.), and the Federal Department for Education and Research (Bernstein Center for Computational Neuroscience Goettingen to T.M. and Bernstein fellowship to A.N.).

18. Merchan-Perez A, Liberman MC (1996) Ultrastructural differences among afferent synapses on cochlear hair cells: Correlations with spontaneous discharge rate. J Comp Neurol 371:208-221.

19. Griesinger $C B$, Richards $C D$, Ashmore JF (2005) Fast vesicle replenishment allows indefatigable signalling at the first auditory synapse. Nature 435:212-215.

20. Naraghi M (1997) T-jump study of calcium binding kinetics of calcium chelators. Cell Calcium 22:255-268.

21. Neher E (1998) Vesicle pools and $\mathrm{Ca}^{2+}$ microdomains: New tools for understanding their roles in neurotransmitter release. Neuron 20:389-399.

22. Zenisek D, Horst NK, Merrifield C, Sterling P, Matthews G (2004) Visualizing synaptic ribbons in the living cell. J Neurosci 24:9752-9759.

23. DiGregorio DA, Peskoff A, Vergara JL (1999) Measurement of action potential-induced presynaptic calcium domains at a cultured neuromuscular junction. J Neurosci 19:7846-7859.

24. Herrington J, Park YB, Babcock DF, Hille B (1996) Dominant role of mitochondria in clearance of large $\mathrm{Ca}^{2+}$ loads from rat adrenal chromaffin cells. Neuron 16:219-228.

25. Zhou Z, Neher E (1993) Mobile and immobile calcium buffers in bovine adrenal chromaffin cells. J Physiol 469:245-273.

26. Wu YC, Tucker T, Fettiplace R (1996) A theoretical study of calcium microdomains in turtle hair cells. Biophys J 71:2256-2275.

27. Matveev V, Sherman A, Zucker RS (2002) New and corrected simulations of synaptic facilitation. Biophys $J$ 83:1368-1373.

28. Borst A, Abarbanel HD (2007) Relating a calcium indicator signal to the unperturbed calcium concentration time-course. Theor Biol Med Model 4:7.

29. Brown AM, Kunze DL, Yatani A (1984) The agonist effect of dihydropyridines on Ca channels. Nature 311:570-572.

30. Martinez-Dunst C, Michaels RL, Fuchs PA (1997) Release sites and calcium channels in hair cells of the chick's cochlea. J Neurosci 17:9133-9144.

31. Khimich D, et al. (2005) Hair cell synaptic ribbons are essential for synchronous auditory signalling. Nature 434:889-894.

32. Roberts WM (1994) Localization of calcium signals by a mobile calcium buffer in frog saccular hair cells. J Neurosci 14:3246-3262.

33. Robertson D, Paki B (2002) Role of L-type $\mathrm{Ca}^{2+}$ channels in transmitter release from mammalian inner hair cells. II. Single-neuron activity. I Neurophysiol 87:27342740 . 


\section{Supporting Information}

\section{Frank et al. 10.1073/pnas.0813213106}

\section{SI Text}

Detailed Description of the Model. To validate result-based assumptions about $\mathrm{Ca}^{2+}$ influx and buffering at the IHC synapse, we used a diffusion-reaction calculator (CalC; public domain; ref. 1) to simulate synaptic $\mathrm{Ca}^{2+}$ signals for a simplified IHC synapse model.

The model (for parameters, see Table S3) included $\mathrm{Ca}^{2+}$ entry into the cytoplasm from a single source and subsequent diffusion as well as reaction with defined buffer species. The model was set up in a 3D geometry (in Cartesian coordinates), with the unitary simulation volume being a cube with an edge length of $100 \mathrm{~nm}$, chosen to be comparable to the experimentally used spatial sampling step size of the microscope's excitation detection volume $(130 \mathrm{~nm})$. The total simulation volume was likewise cubic, with an edge length of $5.1 \mu \mathrm{m}$. The boundary plane harboring the $\mathrm{Ca}^{2+}$ source was considered to represent the plasma membrane and was therefore set to be reflective for all species. At all other boundaries, the concentration was kept constant at the resting level of the respective species.

Because we focus on short stimuli $(20 \mathrm{~ms})$, we neglect $\mathrm{Ca}^{2+}$ removal mechanisms, like extrusion by ATP-driven $\mathrm{Ca}^{2+}$ pumps or uptake into intracellular $\mathrm{Ca}^{2+}$ stores (3). We assumed the presence of $100 \mathrm{Ca}_{\mathrm{V}} 1.3$ channels at an average synapse. The FWHM of the $\mathrm{Ca}^{2+}$ source was set to $300 \mathrm{~nm}$. Activation and deactivation kinetics of the $\mathrm{Ca}^{2+}$ influx, unitary current, and the steady-state open probability were set to values established in or extrapolated from published data. We included EGTA, Fluo-5N, and ATP (at the concentrations used in the experiments) as standard buffers. For the $\mathrm{Ca}^{2+}-$ EGTA reaction, we used the values described in DiGregorio et al. (4), which were determined at the same $\mathrm{pH}$ as our intracellular solution $(\mathrm{pH} 7.0)$. The kinetic parameters for the reaction of $\mathrm{Ca}^{2+}$ and Fluo-5N were taken from unpublished observations, kindly communicated by M. Alp and W.M. Roberts. We did not attempt to simulate the "close to physiological" $\mathrm{Ca}^{2+}$ microdomains (observed in the perforatedpatch configuration after short preloading with Fluo-5N; see Fig. 5 of the main manuscript) because of cooperativity of $\mathrm{Ca}^{2+}$ binding by calretinin (5) and calbindin (6), a feature not implemented in the CalC software. Furthermore, CalC assumes a 1:1 stoichiometry of the calcium-buffer reaction, complicating an implementation of $\mathrm{Ca}^{2+}$ buffers with multiple $\mathrm{Ca}^{2+}$-binding sites present in IHCs [e.g., calretinin, calbindin, or parvalbumin- $\alpha$ (7)]. Note also that for the perforated-patch recordings, only 15-30 s of whole-cell configuration were used for preloading Fluo-5N. Modeling based on Pusch and Neher (8) indicated "wash-out" of a maximum of $17 \%$ of the lightest $\mathrm{Ca}^{2+}$ buffering protein, parvalbumin- $\alpha$, apparent molecular mass of $15 \mathrm{kDa}(9)$, over the longest whole-cell period of $30 \mathrm{~s}$ and at the average series resistance of $9 \mathrm{M} \Omega$.

However, the $\mathrm{Ca}^{2+}$ changes initially predicted by the initial version of the model were faster than the experimental results. This was also observed by Issa and Hudspeth (10) and attributed to the limited scan rate $(500 \mathrm{~Hz})$. Despite a 4 times faster readout in the present study (spot detection compared with line scan), model prediction and result still deviated. This led us to invoke the presence of immobile $\mathrm{Ca}^{2+}$-binding sites for obtaining a better match to the experimentally observed kinetics (Fig. $4 D$ and Fig. S1c) (11). We included two kinds of immobile buffers with comparably low affinities but different kinetics. Both immobile buffer species were distributed evenly throughout the simulation volume. The synaptic $\mathrm{Ca}^{2+}$ signal was simulated for
$41 \mathrm{~ms}$ (1 ms prestimulus, $20 \mathrm{~ms}$ of stimulus, and $20 \mathrm{~ms}$ of poststimulus time).

The output of the program was the free concentration of the simulated species $\left(\mathrm{Ca}^{2+}\right.$ and all buffers) in each unitary simulation volume. We approximated the point-spread function (PSF) of our microscope by a 3D Gaussian function, with their widths set to match the experimentally observed FWHM values of our imaging setup (using 100-nm fluorescent beads; see Table S3). To simulate a spot-detection experiment, we convolved the concentration of Ca-Fluo-5N in the entire simulation volume with the approximated 3D PSF (Fig. S1a) (12). We then took the resulting concentration of Ca-Fluo-5N ([Ca-Fluo-5N] $=$ $[\text { Fluo }-5 \mathrm{~N}]_{\text {total }}-[$ Fluo- $\left.5 \mathrm{~N}]\right)$ in the detection volume as a first output. For simulation of line scan/isochronal spot-detection measurements, we shifted the approximated PSF across the simulation volume and merged the obtained traces to construct a line scan-like image (Fig. S1b). To quantitatively compare simulated data with experiments, we converted the concentration of $\mathrm{Ca}^{2+}$-Fluo5N complex into fluorescence by transforming $[\mathrm{Ca}-\mathrm{Fluo} 5 \mathrm{~N}]$ into baseline-subtracted, baseline-normalized fluorescence $\left[\left(F-F_{0}\right) / F_{0}\right)$, as described in Borst and Abarbanel (13). We found an in situ $F_{\max } / F_{\min }$ ratio of $\approx 50$ for Fluo-5N (in our standard cesium glutamate solution). $F_{\min }$ was determined by dialyzing IHCs with our standard internal solution including 10 mM EGTA. For $F_{\max }$ estimates, we used an internal solution containing $10 \mathrm{mM} \mathrm{Ca}^{2+}$ aiming to saturate the indicator.

Optimizing Beam Position Relative to the $\mathrm{Ca}^{2+}$ Microdomain Center. The majority of spot detection measurements were made at the center of a $\mathrm{Ca}^{2+}$ microdomain as well as at 3 neighboring pixels on each side of the center (130-nm spacing between the pixels, along 1 axis). Subsequent analysis always considered the laser beam position resulting in the largest $\Delta F$.

Here, we describe how further efforts for accurately placing the laser beam into the $\mathrm{Ca}^{2+}$ microdomain's center changed the amplitude variability among $\mathrm{Ca}^{2+}$ microdomains. Substantial variability was observed also after further adjusting the focus to the image plane with the largest $\Delta F$ ("Z-Optimization," $\mathrm{CV}$ of $\Delta F_{\max }=0.65,11 z$-steps: $200 \mathrm{~nm}, 42 \mathrm{Ca}^{2+}$ microdomains in 13 IHCs) and in recordings where the $\mathrm{Ca}^{2+}$ microdomain center was identified by Z-Optimization and bilateral spot displacement in $x$ and $y$ ("3D spot displacement," $\mathrm{CV}$ of $\Delta F_{\max }=0.54,24 \mathrm{Ca}^{2+}$ microdomains in 8 IHCs).

Variability of $\mathrm{Ca}^{2+}$ Microdomain Amplitude Is Similar Within Single Cells and Across Cells. Because certain experimental conditions can slightly vary from cell to cell and day to day (i.e., filling with fluorophore), we sought to check whether those variations contributed considerably to the variability in $\mathrm{Ca}^{2+}$ microdomain amplitude. To this end, we compared the variability across the whole population of $\mathrm{Ca}^{2+}$ microdomains (intercellular variability) and across the $\mathrm{Ca}^{2+}$ microdomains of a single cell (intracellular variability). In the latter analysis, we included cells in which 3 or more microdomains were studied. To have a representative comparison, the intercellular analysis was also performed on this subpopulation. To quantify the variability, we used 2 measures: (i) the $\mathrm{CV}$ for the entire population of $\mathrm{Ca}^{2+}$ microdomain amplitudes and the average $\mathrm{CV}$ calculated from intracellular distributions of $\mathrm{Ca}^{2+}$ microdomain amplitudes (Fig. b), and (ii) a bootstrap measure of relative amplitudes $\left(\Delta F_{\text {spot_1 }} /\right.$ $\Delta F_{\text {spot_2 }}$ for which spot_1 and spot_2 were randomly drawn, either from the whole population, excluding pairs of microdo- 
mains from the same IHC, or from one of the intracellular populations (Fig. S2 $c$ and $d$ ). When the ratio was smaller than 1 , we inverted it.

The results indicate that intracellular variability clearly dominates over variations introduced experimentally between cells and over time. We conclude that the population measure of $\mathrm{Ca}^{2+}$ microdomain amplitude variability is largely representative for variability found between active zones in a single cell.

Intracellular Application of $\mathbf{1 0 0} \boldsymbol{\mu} \mathbf{M}$ Ryanodine. As shown in Fig. 3 $A-C$ and Table S1, we did not observe significant changes in $\Delta F$, the rise and decay kinetics, spatial distribution, the voltage of half-maximal $\Delta F_{\text {activation, }}$ or the variability of $\Delta F_{\max }$. We found a slightly reduced steepness of the voltage dependence of $\Delta F$

1. Kennedy HJ, Meech RW (2002) Fast $\mathrm{Ca}^{2+}$ signals at mouse inner hair cell synapse: A role for $\mathrm{Ca}^{2+}$-induced $\mathrm{Ca}^{2+}$ release. J Physiol 539:15-23.

2. Matveev V, Sherman A, Zucker RS (2002) New and corrected simulations of synaptic facilitation. Biophys J 83:1368-1373.

3. Kennedy $\mathrm{HJ}$ (2002) Intracellular calcium regulation in inner hair cells from neonatal mice. Cell Calcium 31:127-136.

4. DiGregorio D, Peskoff A, Vergara J (1999) Measurement of action potential-induced presynaptic calcium domains at a cultured neuromuscular junction. I Neurosci 19:7846-7859.

5. Faas GC, Schwaller B, Vergara JL, Mody I (2007) Resolving the fast kinetics of cooperative binding: $\mathrm{Ca}^{2+}$ buffering by calretinin. PLoS Biol 5:e311.

6. Berggård T, et al. (2002) Calbindin d28k exhibits properties characteristic of a $\mathrm{Ca}^{2+}$ sensor. J Biol Chem 277:16662-16672.

7. Hackney CM, Mahendrasingam S, Penn A, Fettiplace R (2005) The concentrations of calcium buffering proteins in mammalian cochlear hair cells. J Neurosci 25:7867-7875. [mean "slope" of Boltzmann functions, fit to the individual $\Delta F-V$ curves: $6.7 \pm 1.0 \mathrm{mV}$ (ryanodine) vs. $5.8 \pm 1.1 \mathrm{mV}$ (control), $P<$ 0.05 ; see Table S1] compared with the control condition. The functional significance of this finding remains unclear.

Extracellular Application of $\mathbf{4 0}$ or $\mathbf{2 0} \boldsymbol{\mu} \mathbf{M}$ Ryanodine. Continuous bath applications of either $40 \mu \mathrm{M}$ (using $2 \mathrm{mM}$ EGTA in the pipette) or $20 \mu \mathrm{M}$ (using $0.5 \mathrm{mM}$ EGTA in the pipette) were used. Kennedy and Meech (13) found $20 \mu \mathrm{M}$ sufficient to block CICR in immature IHCs. We did not find significantly different $\mathrm{Ca}^{2+}$ microdomain properties in the presence or absence of ryanodine in IHCs of mice after the onset of hearing (see Fig. S3 and Table S2).

8. Pusch M, Neher E (1988) Rates of diffusional exchange between small cells and a measuring patch pipette. Pflügers Arch 411:204-211.

9. Schmidt H, Arendt O, Brown EB, Schwaller B, Eilers J (2007) Parvalbumin is freely mobile in axons, somata and nuclei of cerebellar Purkinje neurones. J Neurochem 100:727735.

10. Issa NP, Hudspeth AJ (1996) The entry and clearance of $\mathrm{Ca}^{2+}$ at individual presynaptic active zones of hair cells from the bullfrog's sacculus. Proc Natl Acad Sci USA 93:95279532.

11. Wu YC, Tucker T, Fettiplace R (1996) A theoretical study of calcium microdomains in turtle hair cells. Biophys J 71:2256-2275.

12. Shuai J, Parker I (2005) Optical single-channel recording by imaging $\mathrm{Ca}^{2+}$ flux through individual ion channels: Theoretical considerations and limits to resolution. Cell Calcium 37:283-299.

13. Borst A, Abarbanel HDI (2007) Relating a calcium indicator signal to the unperturbed calcium concentration time-course. Theor Biol Med Model 4:7. 

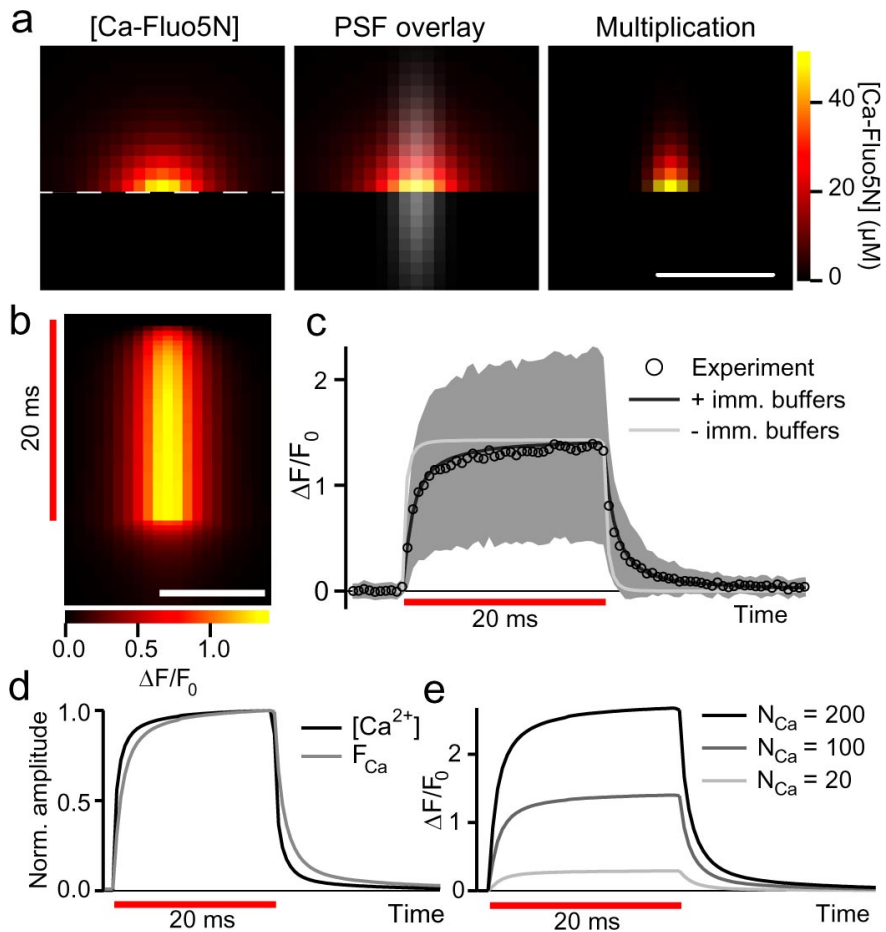

e

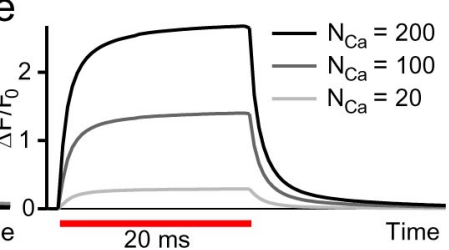

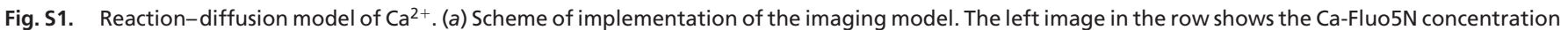

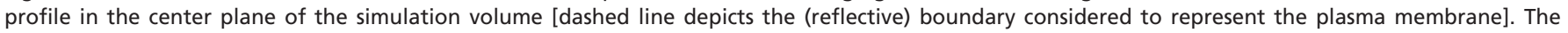

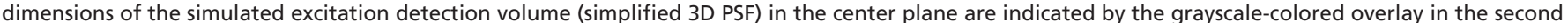

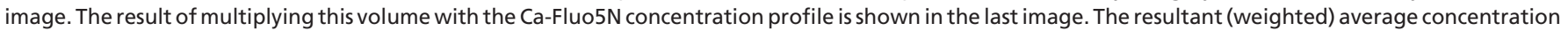

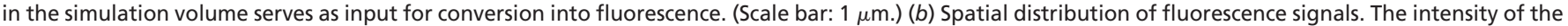

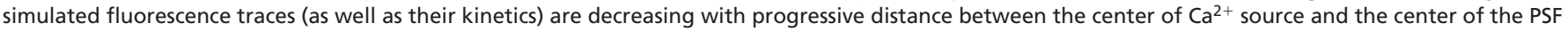
(line scan-like image assembled by shifting the PSF across the $\mathrm{Ca}^{2+}$ source and aligning the resulting fluorescence traces with respect to time). (Scale bar: $1 \mu \mathrm{m}$.)

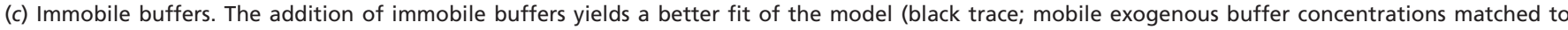

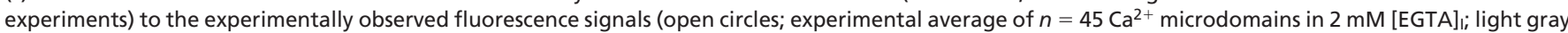

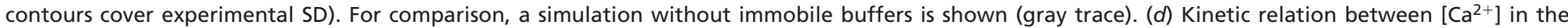

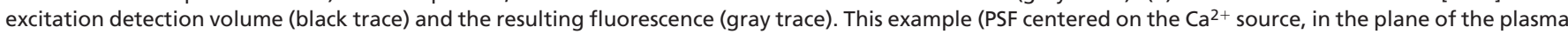

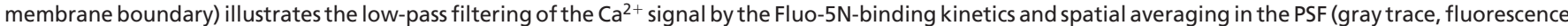

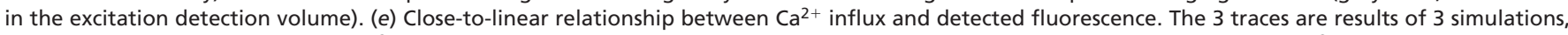
differing only in the amplitude of $\mathrm{Ca}^{2+}$ influx (denominated as different number of channels). In this example, a 10 -fold larger Ca ${ }^{2+}$ influx (16 pA vs. 2 pA) produced $a \approx 9.2$-fold larger fluorescence increase (PSF centered on $\mathrm{Ca}^{2+}$ source). 

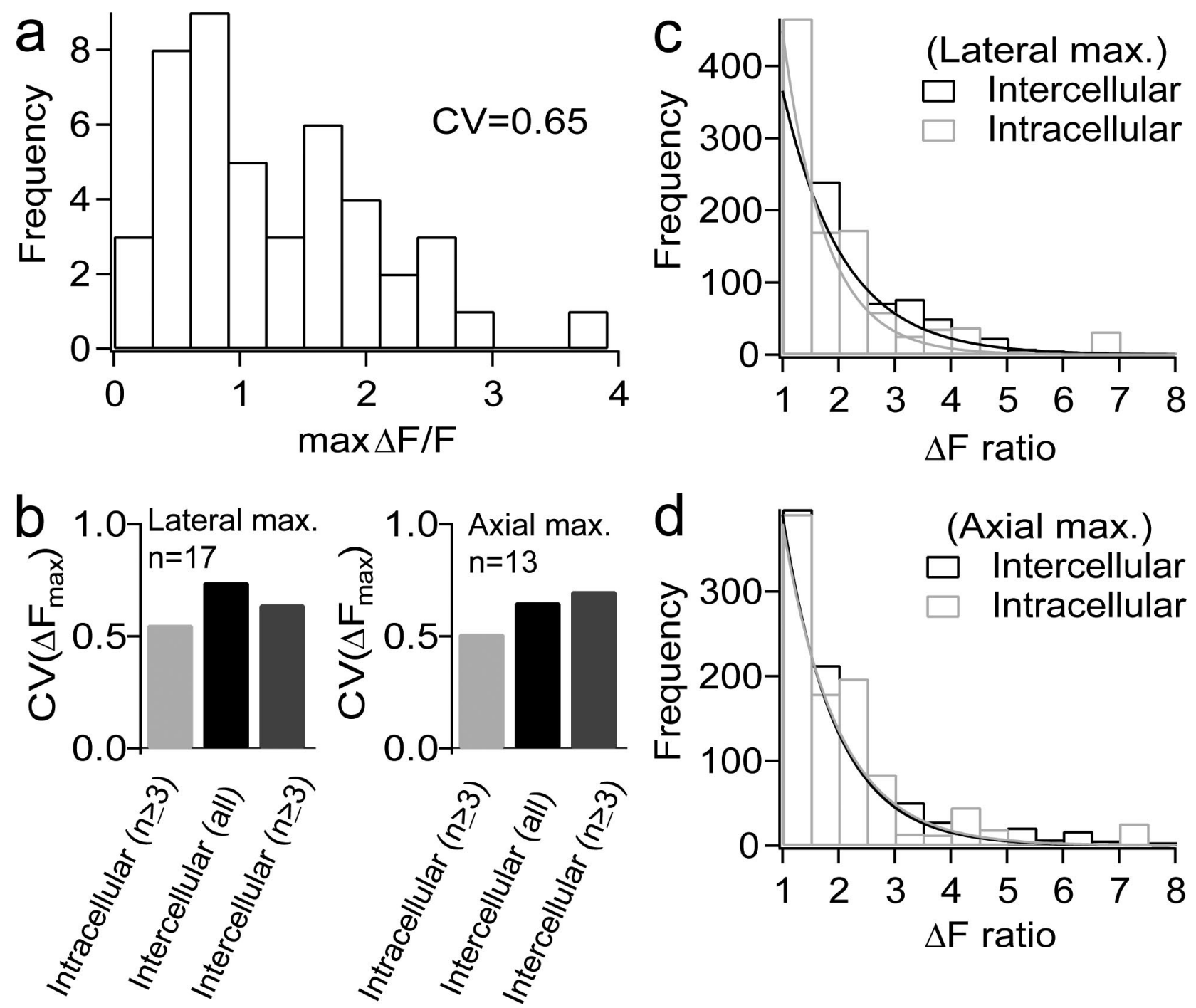

Fig. S2. Intercellular and intracellular variability of $\mathrm{Ca}^{2+}$ microdomain amplitude. (a) Distribution of normalized (to resting fluorescence) fluorescent changes $\left(\Delta F / F_{0}\right)$ in $2 \mathrm{mM}$ EGTA is similar to the distribution of raw fluorescent changes (Fig. $4 B$ ), indicating that local variations in Fluo-5N concentration cannot account for the microdomain variability. (b) Intracellular and intercellular CVs calculated for $\Delta F_{\max }$ in experiments, in which the respective maximum-intensity trace of a set of laterally displaced recording locations (Left; standard spot detection, see Methods) or the respective maximum-intensity trace of a set of axially displaced recording locations was analyzed (Right; spot detection, see Methods). Left bars (light gray) show the average of intracellular CVs (intracellular CVs calculated from $\Delta F_{\max }$ amplitudes of all $\mathrm{Ca}^{2+}$ microdomains within this cell, if $\geq 3 \mathrm{Ca}^{2+}$ microdomains were studied in this particular cell). Middle bars (black) show the population CV of all microdomains within one population (across all cells); right bars (dark gray) show population $\mathrm{CV}$ of all $\mathrm{Ca}^{2+}$ microdomains from cells in which

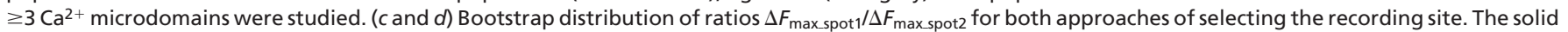
lines represent results of exponential fits (constrained to zero offset). 
a

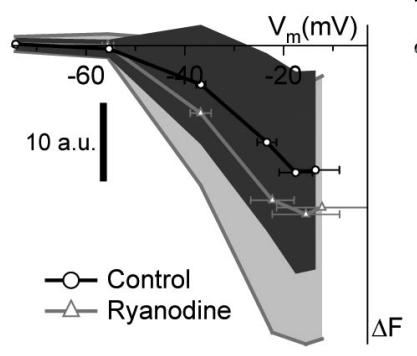

b

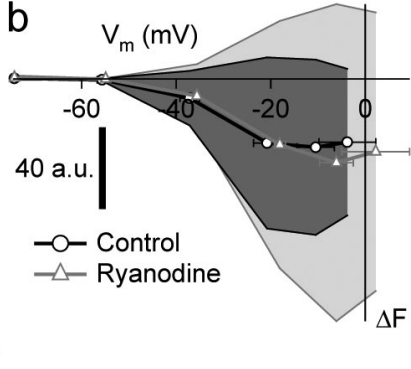

Fig. S3. Voltage dependence of $\mathrm{Ca}^{2+}$ microdomain amplitude in the presence/absence of extracellular ryanodine. (a) Effect of $40 \mu \mathrm{M}$ extracellular ryanodine on $\mathrm{Ca}^{2+}$ microdomain amplitude, with $2 \mathrm{mM} \mathrm{EGTA}$ in $\mathrm{KCl}$-based intracellular solution (see Methods). Mean and SD of $\Delta F$ are shown as a function of membrane potential $\left(V_{\mathrm{m}}\right)$. Data were obtained from spot-detection experiments at the center of $\mathrm{Ca}^{2+}$ microdomains ( $\Delta F$ was averaged over the last $15 \mathrm{~ms}$ of a 20 -ms stimulus obtained as described for Fig. 2E). Overlapping voltage dependence of $\Delta F$ for control (black trace and dark gray area) and ryanodine (gray trace and light gray area) conditions is shown. (b) Effect of $20 \mu \mathrm{M}$ extracellular ryanodine on $\mathrm{Ca}^{2+}$ microdomain amplitude, with $0.5 \mathrm{mM}$ EGTA in KCl-based intracellular solution (see Methods). Mean and SD of $\Delta F$ are shown as a function of membrane potential $\left(V_{\mathrm{m}}\right)$. Data were obtained from spot-detection experiments at the center of $\mathrm{Ca}^{2+}$ microdomains ( $\Delta F$ was averaged over the last $15 \mathrm{~ms}$ of a 20 -ms stimulus obtained as described for Fig. $2 E$ ). Overlapping voltage dependence of $\Delta F$ for control (black trace and dark gray area) and ryanodine (gray trace and light gray area) conditions is shown. 

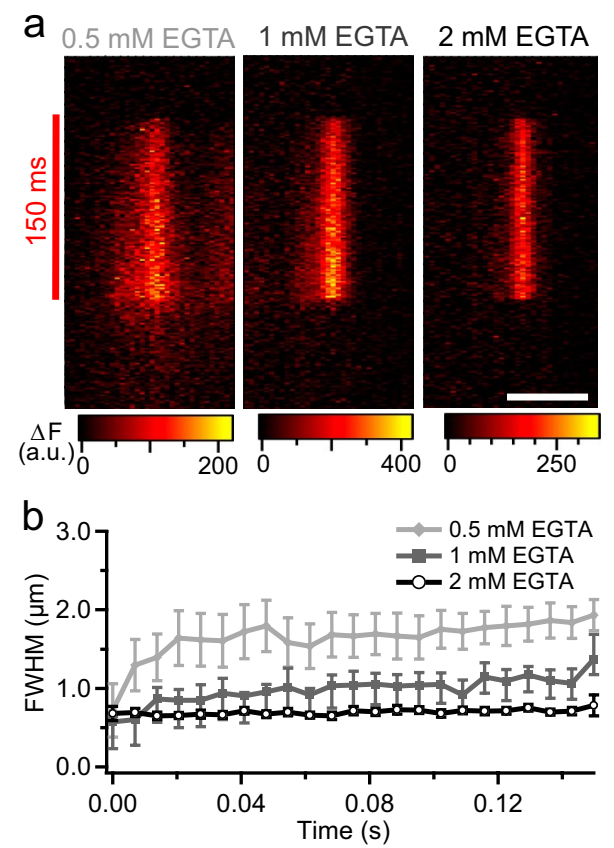

Fig. S4. Time-resolved effects of $\mathrm{Ca}^{2+}$ buffering on spatial spread of $\mathrm{Ca}^{2+}$ microdomains. (a) Representative line scans (red bar depicts timing of 150 ms depolarization to $-7 \mathrm{mV}$ ) for the indicated EGTA pipette concentrations (Scale bar: $2 \mu \mathrm{m}$.) (b) Average ( \pm SEM) FWHM of Ca ${ }^{2+}$ microdomains over time of depolarization (start of depolarization at $0 \mathrm{~ms}$ ). For each $\mathrm{Ca}^{2+}$ microdomain, we split the line scan image into averages of 4 consecutive lines (single line: 1.36 ms; thus, averaged line corresponding to $5.4 \mathrm{~ms}$ ) and fit the average lines with a Gaussian function (0.5 EGTA: $n=5 ; 1$ EGTA: $n=7 ; 2$ EGTA: $n=31$ ). 
Table S1. Average values $( \pm S D)$ for maximum fluorescent amplitude $\left(\Delta F_{\max }\right)$, results of exponential fitting to the maximum-intensity traces from spot-detection measurements, and fits of a Boltzmann function to the fluorescence-voltage relationships

\begin{tabular}{|c|c|c|c|c|c|c|c|c|c|}
\hline Condition & $\Delta F_{\max }, \mathrm{AU}$ & $\tau_{\text {fast (onset) }}, \mathrm{ms}$ & $\begin{array}{c}\tau_{\text {slow (onset) }} \\
\mathrm{ms}\end{array}$ & $\begin{array}{c}A_{\text {fast }} / A_{\text {slowı }} \\
\text { (onset) }\end{array}$ & $\begin{array}{c}\tau_{\text {fast (decay) }} \\
\text { ms }\end{array}$ & $\tau_{\text {slow (decay), }} \mathrm{ms}$ & $\begin{array}{c}A_{\text {fast }} / A_{\text {slow }} \\
\text { (decay) }\end{array}$ & $V_{1 / 2}, \mathrm{mV}$ & Slope, mV \\
\hline \multirow[t]{2}{*}{ Endogenous buffer (14 IHCs) } & $\begin{array}{l}14.1 \pm 9.0 \\
(37 / 37)\end{array}$ & $\begin{array}{l}1.9 \pm 1.3 \\
(30 / 37)\end{array}$ & $\begin{array}{l}15 \pm 11 \\
(17 / 37)\end{array}$ & $\begin{array}{l}1.8 \pm 2.2 \\
(10 / 37)\end{array}$ & $\begin{array}{l}1.5 \pm 1.2 \\
(28 / 37)\end{array}$ & $\begin{array}{l}14 \pm 10 \\
(31 / 37)\end{array}$ & $\begin{array}{l}1.7 \pm 1.2 \\
(22 / 37)\end{array}$ & $\begin{array}{l}-25.9 \pm 13.9 \\
(17 / 33)\end{array}$ & $\begin{array}{l}7.8 \pm 4.8 \\
(17 / 33)\end{array}$ \\
\hline & $C V=0.64$ & $C V=0.65$ & $C V=0.76$ & $C V=1.19$ & $\mathrm{CV}=0.81$ & $C V=0.72$ & $C V=0.69$ & & $C V=0.61$ \\
\hline \multirow[t]{2}{*}{0.5 mM EGTA (15 IHCs) } & $\begin{array}{l}62.1 \pm 38.7 * \\
(24 / 24)\end{array}$ & $\begin{array}{l}1.3 \pm 0.9 \\
(19 / 24)\end{array}$ & $\begin{array}{l}18 \pm 19 \\
(23 / 24)\end{array}$ & $\begin{array}{l}1.1 \pm 1.7^{*} \\
(18 / 24)\end{array}$ & $\begin{array}{l}2.5 \pm 2.1 * \\
(20 / 24\end{array}$ & $\begin{array}{l}17 \pm 11 * \\
(24 / 24)\end{array}$ & $\begin{array}{l}2.6 \pm 1.6^{\dagger} \\
(20 / 24)\end{array}$ & $\begin{array}{l}-32.5 \pm 8.1 \\
(16 / 16)\end{array}$ & $\begin{array}{l}6.2 \pm 1.0 \\
(16 / 16)\end{array}$ \\
\hline & $C V=0.62$ & $C V=0.66$ & $C V=1.02$ & $C V=1.48$ & $C V=0.84$ & $C V=0.65$ & $C V=0.62$ & & $\mathrm{CV}=0.17$ \\
\hline Simulation (0.5 mM EGTA) & & 1.1 & 7 & 0.9 & 1.0 & 6 & 1.1 & & \\
\hline \multirow[t]{2}{*}{2 mM EGTA (17 IHCs) } & $\begin{array}{l}42.8 \pm 31.5^{*} \\
(45 / 45)\end{array}$ & $\begin{array}{l}1.1 \pm 0.5^{*} \\
(44 / 45)\end{array}$ & $\begin{array}{l}14 \pm 9 \\
(26 / 45)\end{array}$ & $\begin{array}{l}2.5 \pm 1.9 * \\
(26 / 45)\end{array}$ & $\begin{array}{l}1.2 \pm 0.9 * \\
(44 / 45)\end{array}$ & $\begin{array}{l}7 \pm 4 \\
(28 / 45)\end{array}$ & $\begin{array}{l}3.9 \pm 2.2^{\dagger} \\
(27 / 45)\end{array}$ & $\begin{array}{l}-30.7 \pm 4.9^{\dagger} \\
(28 / 32)\end{array}$ & $\begin{array}{l}5.9 \pm 1.4 \\
(28 / 32)\end{array}$ \\
\hline & $\mathrm{CV}=0.74$ & $C V=0.45$ & $C V=0.68$ & $C V=0.77$ & $\mathrm{CV}=0.74$ & $C V=0.57$ & $C V=0.56$ & & $\mathrm{CV}=0.23$ \\
\hline Simulation (2 mM EGTA) & & 1.0 & 5 & 2.9 & 0.9 & 4 & 2.2 & & \\
\hline \multirow[t]{2}{*}{10 mM EGTA (7 IHCs) } & $\begin{array}{l}20.1 \pm 13.2 \\
(22 / 22)\end{array}$ & $\begin{array}{l}0.8 \pm 0.5 \\
(22 / 22)\end{array}$ & $\begin{array}{l}8 \pm 5 \\
(4 / 22)\end{array}$ & $\begin{array}{l}3.2 \pm 1.5 \\
(4 / 22)\end{array}$ & $\begin{array}{l}0.7 \pm 0.2 \\
(22 / 22)\end{array}$ & $\begin{array}{l}7 \pm 5 \\
(2 / 22)\end{array}$ & $\begin{array}{l}8.6 \pm 1.0 \\
(2 / 22)\end{array}$ & $\begin{array}{l}-26.5 \pm 6.9 \\
(15 / 15)\end{array}$ & $\begin{array}{l}7.2 \pm 2.9 \\
(15 / 15)\end{array}$ \\
\hline & $C V=0.66$ & $C V=0.58$ & $C V=0.63$ & $C V=0.46$ & $C V=0.27$ & $C V=0.71$ & $C V=0.12$ & & $\mathrm{CV}=0.41$ \\
\hline Simulation (10 mM EGTA) & & 0.6 & - & - & 0.6 & - & - & & \\
\hline \multirow[t]{2}{*}{ Ryanodine (10 IHCs) } & $\begin{array}{l}50.8 \pm 24.4 \\
(15 / 15)\end{array}$ & $\begin{array}{l}1.9 \pm 1.3 \\
(11 / 15)\end{array}$ & $\begin{array}{l}18 \pm 11 \\
(12 / 15)\end{array}$ & $\begin{array}{l}0.6 \pm 0.4 \\
(8 / 15)\end{array}$ & $\begin{array}{l}2.1 \pm 1.3 \\
(13 / 15)\end{array}$ & $\begin{array}{l}41 \pm 62 \\
(15 / 15)\end{array}$ & $\begin{array}{l}1.8 \pm 1.2 \\
(13 / 15)\end{array}$ & $\begin{array}{l}-27.5 \pm 4.9 \\
(12 / 12)\end{array}$ & $\begin{array}{l}6.7 \pm 1.0^{\ddagger} \\
(12 / 12)\end{array}$ \\
\hline & $\mathrm{CV}=0.48$ & $C V=0.68$ & $C V=0.62$ & $\mathrm{CV}=0.7$ & $C V=0.62$ & $C V=1.5$ & $C V=0.66$ & & $C V=0.15$ \\
\hline \multirow[t]{2}{*}{ Control (14 IHCs) } & $\begin{array}{l}59.2 \pm 28.8 \\
(19 / 19)\end{array}$ & $\begin{array}{l}1.6 \pm 0.9 \\
(13 / 19)\end{array}$ & $\begin{array}{l}14 \pm 11 \\
(17 / 19)\end{array}$ & $\begin{array}{l}0.5 \pm 0.4 \\
(11 / 19)\end{array}$ & $\begin{array}{l}2.7 \pm 1.5 \\
(18 / 19)\end{array}$ & $\begin{array}{l}31 \pm 28 \\
(19 / 19)\end{array}$ & $\begin{array}{l}1.6 \pm 0.8 \\
(18 / 19)\end{array}$ & $\begin{array}{l}-27.9 \pm 5.4 \\
(16 / 16)\end{array}$ & $\begin{array}{l}5.8 \pm 1.1 \\
(16 / 16)\end{array}$ \\
\hline & $C V=0.49$ & $C V=0.56$ & $C V=0.79$ & $C V=0.78$ & $C V=0.53$ & $C V=0.90$ & $C V=0.5$ & & $\mathrm{CV}=0.19$ \\
\hline \multirow[t]{2}{*}{ FCCP (10 IHCs) } & $\begin{array}{l}94.6 \pm 98.5 \\
(21 / 21)\end{array}$ & $\begin{array}{l}5.3 \pm 4.3 \\
(17 / 21)\end{array}$ & $\begin{array}{l}97 \pm 46^{\S} \\
(21 / 21)\end{array}$ & $\begin{array}{l}0.7 \pm 0.7 \\
(17 / 21)\end{array}$ & $\begin{array}{l}17.9 \pm 11.1 \\
(18 / 21)\end{array}$ & $\begin{array}{l}108 \pm 48 \\
(21 / 21)\end{array}$ & $\begin{array}{l}2.4 \pm 2.6^{*} \\
(18 / 21)\end{array}$ & & \\
\hline & $C V=1.04$ & $C V=0.81$ & $C V=0.48$ & $C V=0.94$ & $C V=0.62$ & $C V=0.45$ & $C V=1.10$ & & \\
\hline \multirow[t]{2}{*}{ Control (7 IHCs) } & $\begin{array}{l}79.7 \pm 107 \\
(11 / 11)\end{array}$ & $\begin{array}{l}9.4 \pm 7 \\
(8 / 11)\end{array}$ & $\begin{array}{l}152 \pm 40 \\
(9 / 11)\end{array}$ & $\begin{array}{l}2.3 \pm 5.4 \\
(8 / 11)\end{array}$ & $\begin{array}{l}22.0 \pm 10.7 \\
(8 / 11)\end{array}$ & $\begin{array}{l}143 \pm 63 \\
(9 / 11)\end{array}$ & $\begin{array}{l}0.5 \pm 0.4 \\
(8 / 11)\end{array}$ & & \\
\hline & $\mathrm{CV}=1.34$ & $\mathrm{CV}=0.74$ & $\mathrm{CV}=0.26$ & $C V=2.39$ & $C V=0.48$ & $C V=0.44$ & $C V=0.69$ & & \\
\hline \multirow[t]{2}{*}{ BayK (5 IHCs) } & $\begin{array}{l}59.2 \pm 52 \\
(16 / 16)\end{array}$ & $\begin{array}{l}3.8 \pm 3.4 \\
(14 / 16)\end{array}$ & $\begin{array}{l}183 \pm 113 \\
(16 / 16)\end{array}$ & $\begin{array}{l}1.8 \pm 2.7 \\
(14 / 16)\end{array}$ & $\begin{array}{l}5.1 \pm 5.4 \\
(15 / 16)\end{array}$ & $\begin{array}{l}45 \pm 62 \\
(15 / 16)\end{array}$ & $\begin{array}{l}4.1 \pm 3.0 \\
(14 / 16)\end{array}$ & & \\
\hline & $C V=0.87$ & $\mathrm{CV}=0.90$ & $C V=0.62$ & $C V=1.5$ & $C V=1.06$ & $C V=1.40$ & $C V=0.74$ & & \\
\hline
\end{tabular}

If applicable [i.e., normality of (Jarque-Bera test) and equal variances between ( $F$ test) the 2 samples], an independent 2 -sample $t$ test was used ( $t$, $P<0.05$;

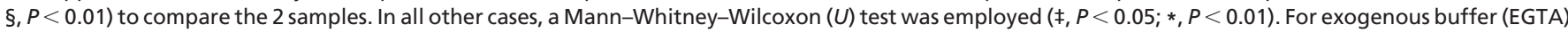
experiments, the $0.5 \mathrm{mM}$ was compared to the $2 \mathrm{mM}$ condition, and the $2 \mathrm{mM}$ to the $10 \mathrm{mM}$ condition, respectively. Numbers in parentheses indicate number

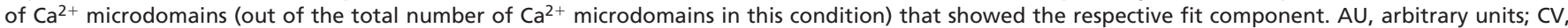

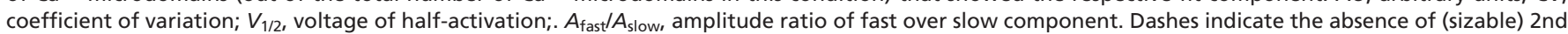
(slow) exponential component in results of $10 \mathrm{mM}$ EGTA simulations. 
Table S2. Summary of voltage dependence of $\mathrm{Ca}^{2+}$ microdomain amplitude in the presence/absence of extracellular ryanodine

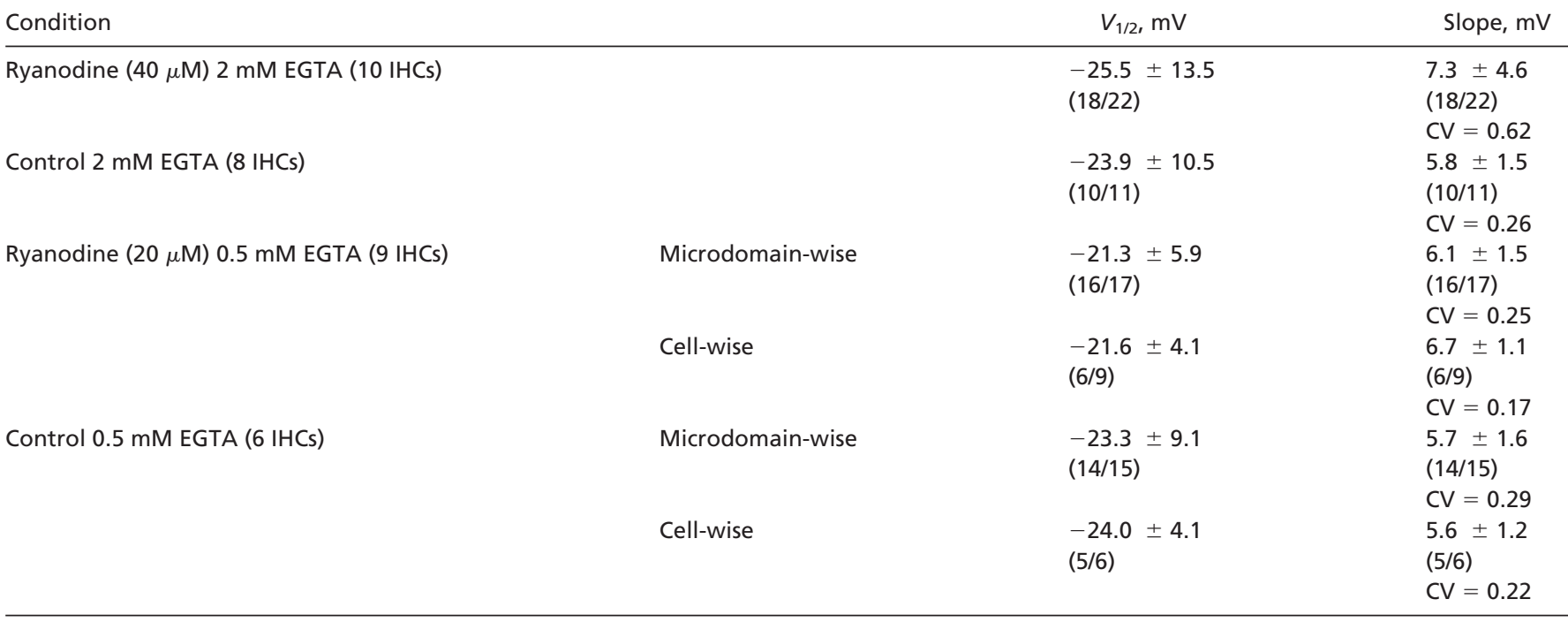

Average values $( \pm S D)$ for fits of a Boltzmann function to the fluorescence-voltage relationships. If applicable, [i.e., normality of (Jarque-Bera test) and equal variances between ( $F$ test) the 2 samples] an independent 2-sample $t$ test was used to compare the 2 samples. In all other cases, a Mann-Whitney-Wilcoxon ( $U$ ) test was employed. Numbers in parentheses indicate number of $\mathrm{Ca}^{2+}$ microdomains (out of the total number of $\mathrm{Ca}^{2+}$ microdomains in this condition) that showed the respective fit component. CV, coefficient of variation; $V_{1 / 2}$, voltage of half-activation. 
Table S3. Parameters used in simulations

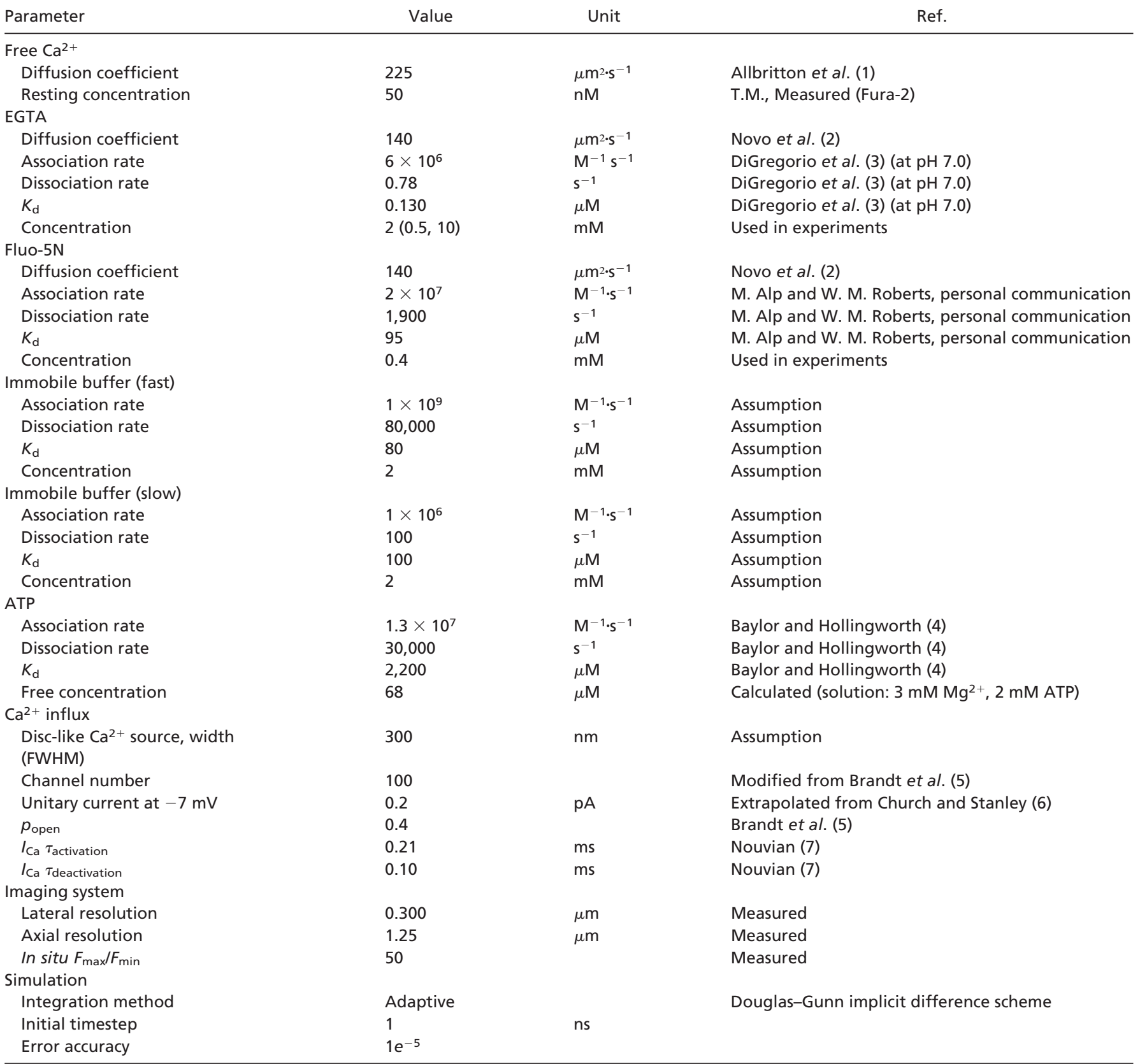

1. Allbritton NL, Meyer T, Stryer, L (1992) Range of messenger action of calcium ion and inositol 1,4,5-trisphosphate. Science 258:1812-1815.

2. Novo D, Difranco M, Vergara JL (2003) Comparison between the predictions of diffusion-reaction models and localized Ca ${ }^{2+}$ transients in amphibian skeletal muscle fibers. Biophys $J$ 85:1080-1097.

3. DiGregorio D, Peskoff A, Vergara J (1999) Measurement of action potential-induced presynaptic calcium domains at a cultured neuromuscular junction. J Neurosci 19:7846-7859.

4. Baylor SM, Hollingworth S (1998) Model of sarcomeric $\mathrm{Ca}^{2+}$ movements, including ATP $\mathrm{Ca}^{2+}$ binding and diffusion, during activation of frog skeletal muscle. J Gen Physiol 112:297-316.

5. Brandt A, Khimich D, Moser T (2005) Few Cav1.3 channels regulate the exocytosis of a synaptic vesicle at the hair cell ribbon synapse. J Neurosci 25:11577-11585.

6. Church PJ, Stanley EF (1996) Single I-type calcium channel conductance with physiological levels of calcium in chick ciliary ganglion neurons. J Physiol 496:59-68.

7. Nouvian R (2007) Temperature enhances exocytosis efficiency at the mouse inner hair cell ribbon synapse. J Physio/ 584:535-542. 


\title{
3. Tuning of Synapse Number, Structure and Function in the Cochlea
}

\author{
Meyer, A.C.*, Frank, T.*, Khimich, D.*, Hoch, G., Riedel, D., Chapochnikov, \\ N.M., Yarin, Y.M., Harke, B., Hell, S.W., Egner, A. and Moser, T. (2009) \\ * These authors contributed equally to the work
}

Nat Neurosci, 12:444-53.

http://www.nature.com/neuro/journal/v12/n4/abs/nn.2293.html

doi:10.1038/nn.2293

Author contributions (as appearing in the paper):

The study was designed by T.M., A.C.M., A.E. and T.F. The experimental work was performed by A.C.M., T.F., D.K., D.R., G.H., N.M.C., Y.M.Y. and B.H. S.W.H. co-developed the super-resolution microscopes.

Detailed author contributions of T. Frank:

- Experimental work and analysis:

- $\mathrm{Ca}^{2+}$ current recordings/analysis (Fig. 4d; Supplementary Fig. 6)

- Non-stationary fluctuation analysis (Fig. 4e,f; Supplementary Table 2)

$\circ \mathrm{Ca}^{2+}$ imaging/analysis (Fig. 5; Fig. 6a-e; Table 2; Supplementary Table 3)

- Preparation of the manuscript (together with T.M. and A.C.M)

\section{Copyright notice:}

This material is reprinted by permission from the Nature Publishing Group in accordance to their copyright and licensing regulations (License-Nr. 2390330030321). Reprinted by permission from Macmillan Publishers Ltd: Nature Neuroscience, (C) owned by the authors, 2009. 


\title{
Tuning of synapse number, structure and function in the cochlea
}

\author{
Alexander C Meyer ${ }^{1,6}$, Thomas Frank ${ }^{1,6}$, Darina Khimich ${ }^{1,6}$, Gerhard Hoch ${ }^{1}$, Dietmar Riedel ${ }^{2}$, \\ Nikolai M Chapochnikov ${ }^{1,3}$, Yury M Yarin ${ }^{1,4}$, Benjamin Harke ${ }^{5}$, Stefan W Hell ${ }^{5}$, \\ Alexander Egner ${ }^{5}$ \& Tobias Moser ${ }^{1,3}$
}

\begin{abstract}
Cochlear inner hair cells (IHCs) transmit acoustic information to spiral ganglion neurons through ribbon synapses. Here we have used morphological and physiological techniques to ask whether synaptic mechanisms differ along the tonotopic axis and within IHCs in the mouse cochlea. We show that the number of ribbon synapses per IHC peaks where the cochlea is most sensitive to sound. Exocytosis, measured as membrane capacitance changes, scaled with synapse number when comparing apical and midcochlear IHCs. Synapses were distributed in the subnuclear portion of IHCs. High-resolution imaging of IHC synapses provided insights into presynaptic $\mathrm{Ca}^{2+}$ channel clusters and $\mathrm{Ca}^{2+}$ signals, synaptic ribbons and postsynaptic glutamate receptor clusters and revealed subtle differences in their average properties along the tonotopic axis. However, we observed substantial variability for presynaptic $\mathrm{Ca}^{2+}$ signals, even within individual IHCs, providing a candidate presynaptic mechanism for the divergent dynamics of spiral ganglion neuron spiking.
\end{abstract}

After processing by the mammalian ear's exquisite micromechanics and mechanoelectrical transduction, acoustic information is encoded at the afferent synapses of IHCs with high temporal precision ${ }^{1,2}$. Presynaptic active zones of IHCs contain a synaptic ribbon, a multiprotein structure that tethers synaptic vesicles ${ }^{3,4}$ and ensures a large pool of readily releasable vesicles ${ }^{5-8}$. Stimulus-secretion coupling is governed by $\mathrm{Ca}_{V} 1.3 \mathrm{~L}$-type $\mathrm{Ca}^{2+}$ channels ${ }^{9,10}$ that tightly control the release of glutamate from nearby fusion-competent vesicles ${ }^{2,8,11}$ onto postsynaptic AMPA receptors ${ }^{12,13}$ on the unbranched peripheral axon of the bipolar spiral ganglion neuron $(\mathrm{SGN})^{14}$. As a result, each SGN receives input from only one IHC active zone, while each IHC drives several SGNs.

Whether and how the number and properties of afferent synapses of the cochlea are 'tuned' for optimal sound encoding remains an important question (refs. 15,16, for example). The frequency selectivity of SGNs is primarily determined by the location of the innervated IHC on the cochlea's tonotopic axis, providing a place code for frequency (refs. 17,18, for example). In addition, studies of small samples of synapses from distinct cochlear regions have indicated that the innervation density varies along the length of the cochlea ${ }^{19-21}$. Moreover, it has been shown that SGNs covering a narrow frequency range differ markedly in spontaneous and evoked firing rates, sound threshold and dynamic range (for example, refs. 22,23) and that they collectively encode a large range of sound pressures. It is generally believed, but not yet directly proven, that each IHC makes contact with such physiologically diverse SGNs. If true, the heterogeneity of SGN dynamics could be caused by pre- and postsynaptic mechanisms $7,19,24,25$. Pioneering work on the cat cochlea suggested that lowspontaneous-rate SGNs preferentially contact active zones with large or even multiple synaptic ribbons at the neural side of IHCs (toward the modiolus), whereas high spontaneous rate SGNs are driven by small, 'simple' synapses at the abneural IHC side (toward the outer hair cells ${ }^{24}$.

Here we have used patch-clamp, confocal imaging of IHC presynaptic $\mathrm{Ca}^{2+}$ signals; confocal, $4 \mathrm{Pi}^{26,27}$ and stimulated emission depletion (STED) ${ }^{28}$ microscopy of immunolabeled synapses; and electron microscopy to characterize the distribution of afferent synapses as well as their structure and function at different tonotopic regions of the cochlea. Having investigated thousands of synapses in hundreds of IHCs, we provide a continuous representation of synapse number per IHC along the entire mouse cochlea, and we show that synapse density parallels the neuronal population audiogram. Using STED microscopy, we provide optical, nanometer-scale measurements of individual clusters of presynaptic $\mathrm{Ca}^{2+}$ channels and postsynaptic AMPA receptors. Whereas average structural and functional synapse properties varied only slightly along the cochlea's tonotopic axis, we found considerable heterogeneity of presynaptic $\mathrm{Ca}^{2+}$ signals among the synapses in IHCs in a given region.

${ }^{1}$ InnerEarLab, Department of Otolaryngology and Center for Molecular Physiology of the Brain, University of Göttingen, Göttingen, Germany. ${ }^{2}$ Laboratory of Electron Microscopy, Max-Planck-Institute for Biophysical Chemistry, Göttingen, Germany. ${ }^{3}$ Bernstein Center for Computational Neuroscience, University of Göttingen, Göttingen, Germany. ${ }^{4}$ Clinic of Otorhinolaryngology, Department of Medicine, Technical University of Dresden, Dresden, Germany. ${ }^{5}$ Department of NanoBiophotonics, Max-PlanckInstitute for Biophysical Chemistry, Göttingen, Germany. ${ }^{6}$ These authors contributed equally to the work. Correspondence should be addressed to T.M. (tmoser@gwdg.de) or A.E. (aegner@gwdg.de). 
Figure 1 The number of afferent synapses per $\mathrm{IHC}$ co-varies with ABR threshold along the tonotopic axis. (a) Projections of confocal stacks of immunostained mouse IHC afferent synapses at different tonotopic locations (red, anti-CtBP2/ RIBEYE; green, anti-GluR2/3; blue, Hoechst 34580 nuclear stain). Percentages indicate the normalized location of the imaged IHCs in the cochlea (0\%, apical end; $100 \%$, basal end). Center: montage of the low-magnification view of fragments of the full explanted organ of Corti. Red circles, locations of the confocal images. (b) Synaptic cochleograms and tone-burst ABR audiograms of NMRI and C57BL/6 mice (together, more than 15,000 synapses) overlaid by relating distance to cochlear apex to the tonotopic map of mice of strain CBA (top axis ${ }^{18}$ ). NMRI mice: red open circles, number of synapses per IHC (26 ears, P15-P20); red filled circles, ABR threshold (average \pm s.e.m., ten ears from six mice). C57BL/6: black open squares, number of synapses per IHC (two ears, P30); connected black filled squares, ABR threshold. Continuous black line: fit of a quadratic function to the collective data set of both mouse lines.

(c) Synaptic cochleograms (circles): number of synapses per IHC (more than 10,000 synapses in six ears from four P28 gerbils), plotted against a tonotopic map (taken from ref. 29) and frequencydependent compound action potentials (CAP) thresholds (from ref. 29) (red line). Continuous black line: fit of a quadratic function to the data.
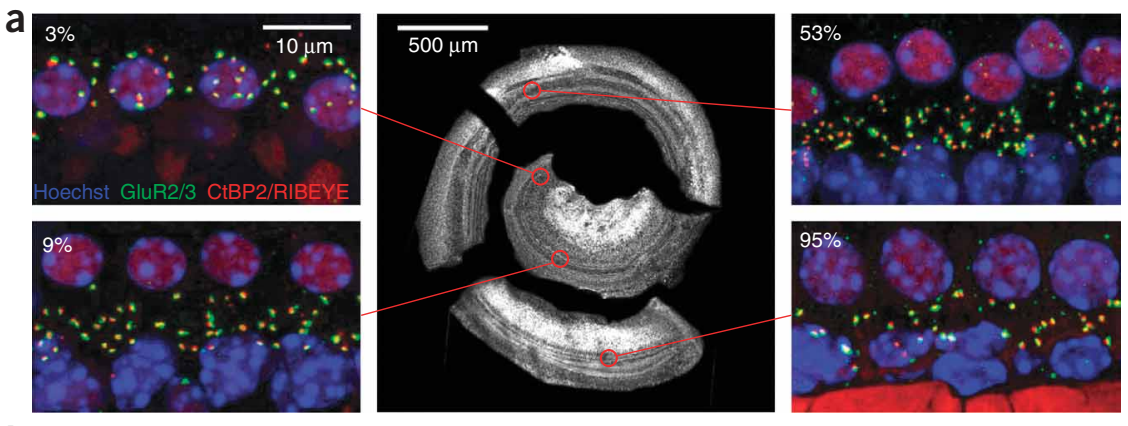

b Mouse

$$
\text { C Gerbil }
$$

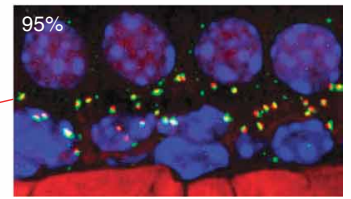

Frequency
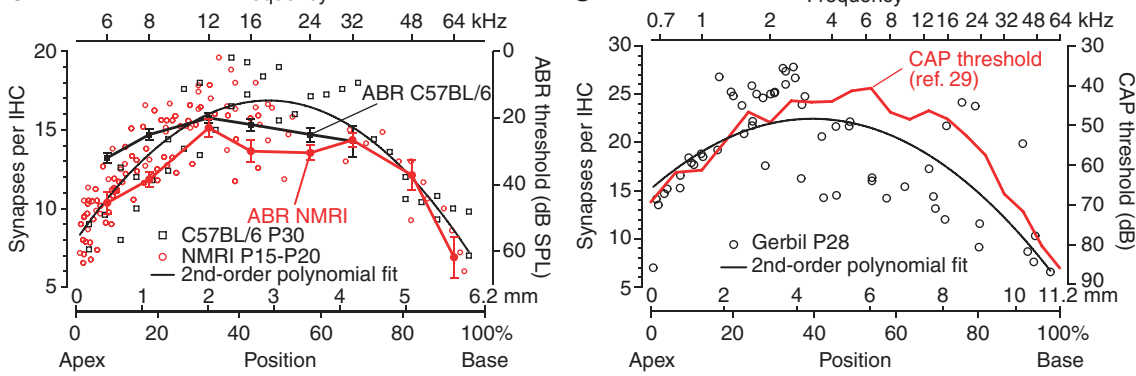

\section{RESULTS}

\section{Tonotopic synapse density map and subcellular distribution}

First, we assessed the number of ribbon synapses per IHC throughout the entire cochlea (creating a synaptic cochleogram) of mice (Fig. 1a,b; high-frequency hearing) and gerbils (Fig. 1c and Supplementary Fig. 1 online; low-frequency hearing) by confocal microscopy of immunolabeled, whole-mount organs of Corti. The microdissected parts of the organ of Corti were aligned for measuring the distance of a given synapse to the apex of the cochlea (see Methods; Fig. 1a). We identified IHC ribbon synapses as colocalized spots of presynaptic ribbons (using antibody to transcription factor CtBP2 sharing homologous domain with RIBEYE, the main protein component of the synaptic ribbon) and postsynaptic GluR2/3 (labeling the AMPA receptor clusters) immunofluorescence in stacks of confocal sections, and divided their count by the number of IHC nuclei ${ }^{6}$. The study used hearing mice (as determined by auditory brainstem responses (ABR)) of two wild-type laboratory strains $(\mathrm{C} 57 \mathrm{BL} / 6 \mathrm{~N}$, postnatal day $(\mathrm{P}) 30$; and NMRI, P15-P20). We approximated their collective synaptic cochleograms by a quadratic function (Fig. 1b) to describe synapse number per IHC along the tonotopic axis (see Supplementary Table 1 online for fit coefficients). In gerbils (P28), we counted only synaptic ribbons because we were not able to stain postsynaptic glutamate receptor clusters reliably (Fig. 1c; Supplementary Fig. 1 and Supplementary Table 1). The synaptic cochleograms were then related to hearing thresholds as estimated by ABR recordings (Fig. 1b; mice were thereafter used for immunohistochemistry) or compound action potentials ${ }^{24}$ (Fig. 1c) using published place-frequency maps ${ }^{18,29}$. For both species, the synaptic cochleograms peaked within
Figure 2 Spatial distribution of afferent synapses within IHCs of one tonotopic region. (a) Cartoon illustrating the analysis and the use of cylindrical coordinates for descriptions of synapse position. (b) Four en face views of six midcochlear IHCs and their afferent synapses (red dots), overlaid after normalization of synapse coordinates in the $z$ axis (from the center of the nucleus to the basal end) and in the radius, $r$ (according to the width at nuclear level) of each IHC. Scale bars, $5 \mu \mathrm{m}$. (c) Histogram shows synapse number as a function of axial distance from the center of the cell's nucleus $(z)$ for a total of twenty-three midcochlear IHCs. (d) Polar scatter plot of 373 synapses mapped in cylindrical coordinates (radius $(r)$ and angle $(\theta)$ are displayed). (e) Polar histogram of synapse density in $36^{\circ}$ sectors. The sum of all 10 sectors equals the average synapse number of 16.2 per cell. (f) Histogram of the nearest neighbor distance distribution of 373 synapses.
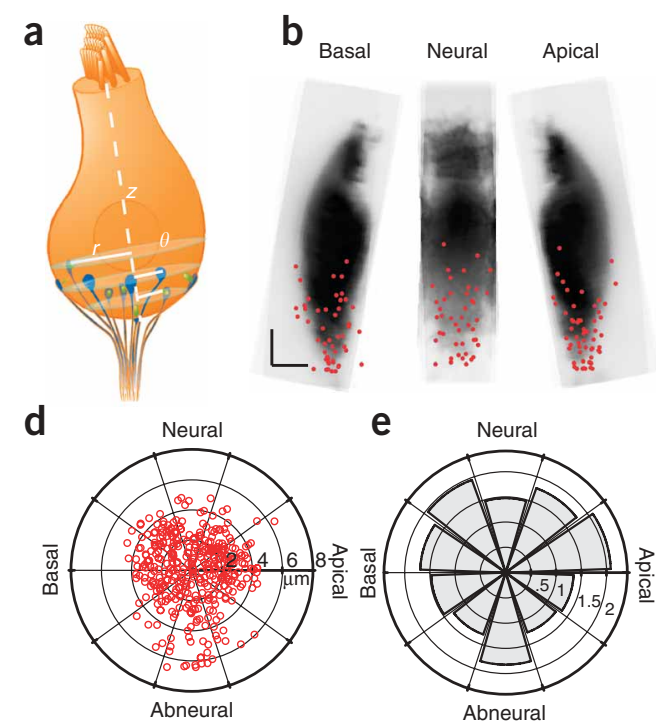

C
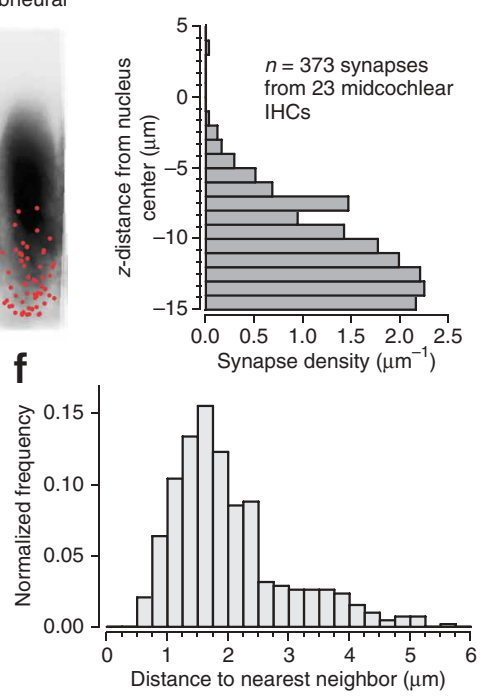

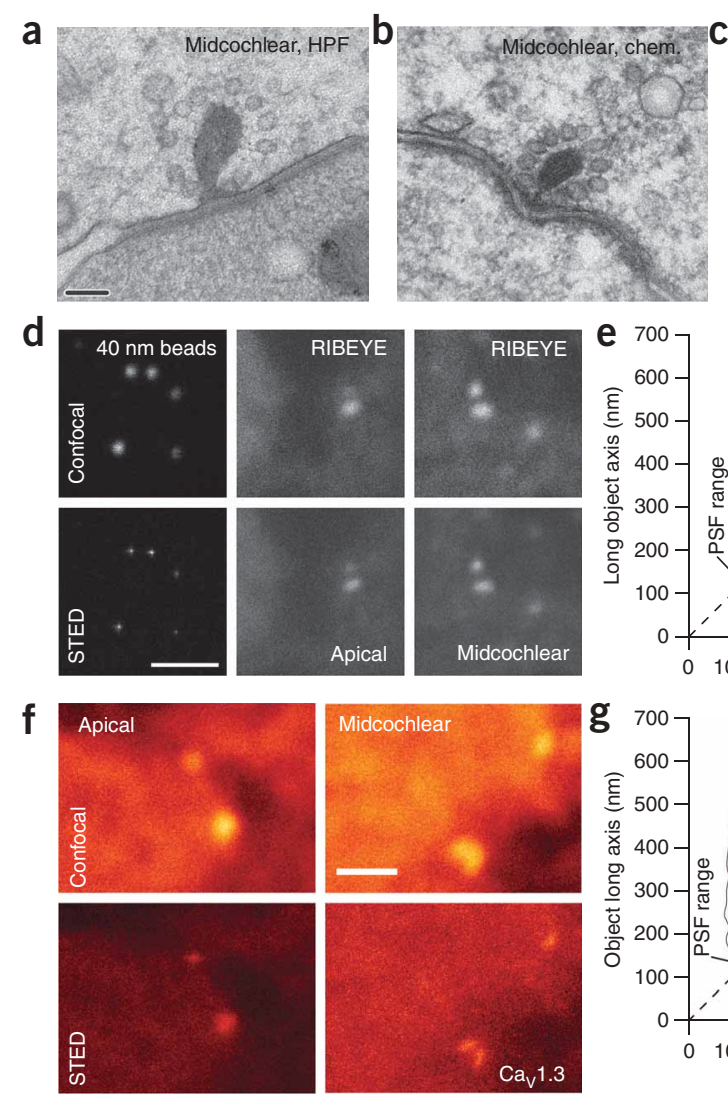

h Apical
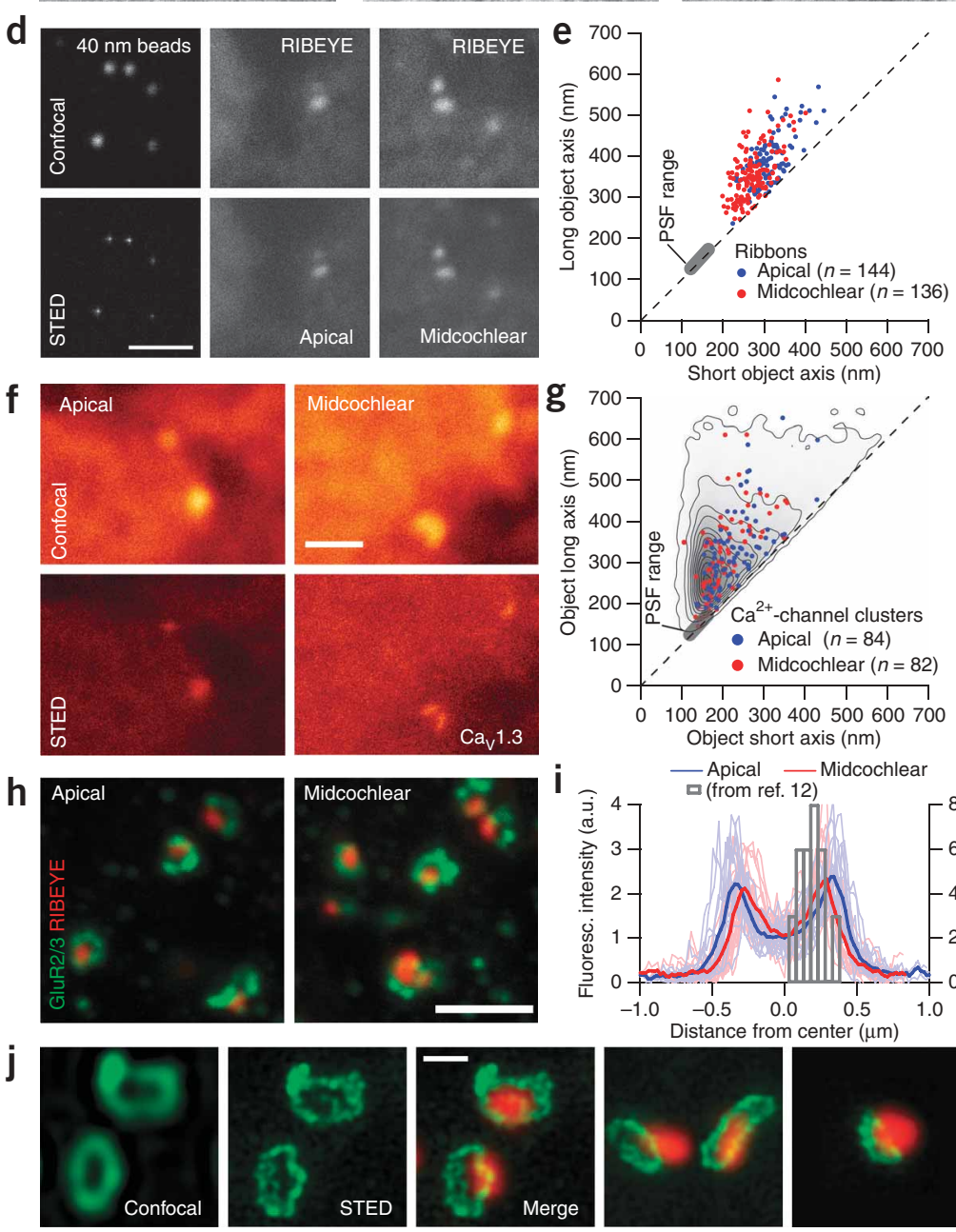

Object short axis (nm)
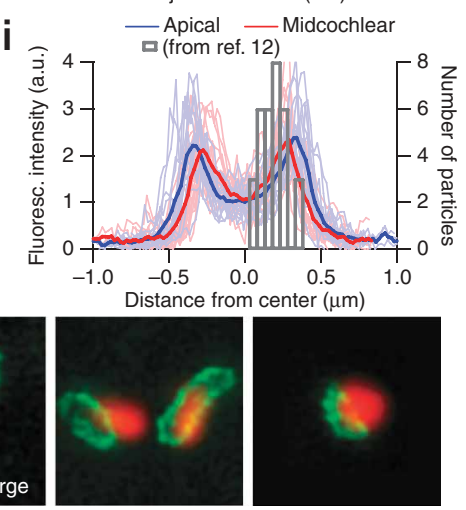

Figure 3 Ribbon synapse morphology in the apical cochlea $(100-400 \mu \mathrm{m})$ and mid-cochlea $(1,300-2,100 \mu \mathrm{m})$ of the mouse (P16-P21). (a-c) Representative electron micrographs of midcochlear (a,b) and apical (c) synapses, after either high-pressure rapid freeze and freezesubstitution (HPF, a) or aldehyde fixation (chem., b and c). Scale bar, $100 \mathrm{~nm}$. (d) Representative confocal (top) and STED (bottom) images of 40-nm beads (left) and fluorescently labeled synaptic ribbons of apical (middle) and midcochlear (right) IHCs. Scale bar, $2 \mu \mathrm{m}$. (e) Long versus short axes for apical (blue) and midcochlear (red) ribbons. Grey bar: resolution limit of the STED microscope in front (lower boundary) and behind (upper boundary) the sample. (f) Representative confocal (top) and STED (bottom) images of immunolabeled Cav1.3 clusters of an apical and a midcochlear IHC imaged 15-20 $\mu \mathrm{m}$ deep in the sample. Scale bar, $1 \mu \mathrm{m}$. The double cluster in the midcochlear IHC was associated with a very large RIBEYE signal (not shown). (g) Long versus short axes for apical (blue) and midcochlear Cav1.3 clusters (red). Expected density (Supplementary Fig. 5) overlaid as contour plot. (h-j) Imaging of postsynaptic AMPA receptor clusters (green, GluR2/3; red, RIBEYE) contacting apical and midcochlear IHCs. (h) Ring-like appearance of clusters oriented en face. Scale bar, $2 \mu \mathrm{m}$. (i) Fluorescence (fluoresc.) profiles from STED en face views of apical (blue) and midcochlear (red) clusters. Profiles were centered at half the distance of the two side-peaks for alignment. Gray bars, counts of immunogold particles as a function of distance from synapse center (rat IHC, taken from ref. 12); a.u., arbitrary units. (j) Two leftmost panels: representative deconvolved $x y$-confocal and STED sections of clusters. Middle panel: same cluster overlaid with confocal image of ribbon. Scale bar, $500 \mathrm{~nm}$. Two rightmost panels: more synapses.

along $\theta$. This is exemplified in views from all four sides of six overlaid midcochlear IHCs (Fig. 2b). We further described the synapse distribution as functions of $z$-position (Fig. 2c) and angle $\theta$ (Figs. 2d,e). The distribution of the three-dimensional nearest

the most sensitive region of the cochlea at $\sim 17$ (mice) or $\sim 24$ (gerbils) synapses per IHC and declined toward the cochlear apex and base.

Next, we studied the synapse distribution within apical (distance to cochlear apex $200 \pm 100 \mu \mathrm{m}, n=2$ mice; Supplementary Fig. 2 online) and midcochlear (1,700 $\pm 400 \mu \mathrm{m}, n=4$ mice; Fig. 2) IHCs. Stacks of confocal images obtained from organs of Corti immunolabeled for CtBP2/RIBEYE and calbindin-28k, a $\mathrm{Ca}^{2+}$-binding protein marking the IHC cytosol, were aligned according to the tonotopic axis (indicated by the row of IHCs) to identify synaptic ribbons and IHC nuclei (see Methods). Synapses were assigned to IHCs based on their distance to the center of the nearest IHC's nucleus. Data were discarded if assignments were ambiguous. The position of each synapse was expressed in cylindrical coordinates ( $z$-axial position, with $z=0$ at the center of the nucleus; radius $r$ and angle $\theta$ as illustrated in Fig. 2a) after normalizing the distance between the center of the nucleus and the basal end of each IHC to the respective mean value of all analyzed IHCs. We observed a strong base-to-apex decline in synapse abundance from the base of the IHC to its apex and a rather uniform distribution neighbor distance had a mean of $\sim 2 \mu \mathrm{m}$ and did not show obvious higher-order peaks (Fig. 2f, estimated before normalization of cell dimensions). The synapse distribution was similar for IHCs in the apex of the cochlea, except for a tendency of synapses to accumulate in the apical and basal IHC sectors (Supplementary Fig. 2).

\section{Synapse morphology as a function of tonotopic position}

The size of the synaptic ribbon largely determines the vesicle complement of each synapse (reviewed in ref. 3) and was previously used to approximate the area of the active zone over which $\mathrm{Ca}^{2+}$ channels are distributed $^{30,31}$. The size of postsynaptic AMPA receptor clusters is a key determinant of synaptic strength (for example, refs. 32,33). Thus, we explored IHC synapse morphology in apical and midcochlear regions using high-resolution fluorescence microscopy of immunolabeled IHC synapses, as well as electron microscopy.

Electron microscopy was performed on ultrathin sections of first apical turns of cochleae. First, we compared the synaptic ultrastructure after (i) chemical immersion fixation or (ii) high-pressure rapid freeze 
Table 1 Morphology of ribbons synapses in apical and midcochlear IHCs

\begin{tabular}{|c|c|c|c|}
\hline & Apical (mean \pm s.d.) & Midcochlear (mean \pm s.d.) & $P$-value \\
\hline Cav1.3 cluster long axis (STED) & $327 \pm 95 \mathrm{~nm}(n=84)$ & $320 \pm 97 \mathrm{~nm}(n=82)$ & $P=0.40$ \\
\hline Cav1.3 cluster short axis (STED) & $228 \pm 59 \mathrm{~nm}(n=84)$ & $208 \pm 55 \mathrm{~nm}(n=82)$ & $P<0.01$ \\
\hline Ribbon long axis (STED) & $379 \pm 62 \mathrm{~nm}(n=144)$ & $357 \pm 63 \mathrm{~nm}(n=136)$ & $P<0.001$ \\
\hline Ribbon long axis (EM) & $228 \pm 60 \mathrm{~nm}(n=29)$ & $223 \pm 59 \mathrm{~nm}(n=37)$ & $P=0.74$ \\
\hline Ribbon short axis (STED) & $299 \pm 46 \mathrm{~nm}(n=144)$ & $273 \pm 39 \mathrm{~nm}(n=136)$ & $P<0.001$ \\
\hline Ribbon short axis (EM) & $118 \pm 27 \mathrm{~nm}(n=29)$ & $117 \pm 36 \mathrm{~nm}(n=37)$ & $P=0.86$ \\
\hline Ribbon cross-section (EM) & $21,337 \pm 8,609 \mathrm{~nm}^{2}(n=29)$ & $20,495 \pm 9,795 \mathrm{~nm}^{2}(n=37)$ & $P=0.28$ \\
\hline Num $_{\mathrm{SV}}<50 \mathrm{~nm}$ from ribbon (EM) & $15.1 \pm 3.5(n=27)$ & $12.7 \pm 3.7(n=34)$ & $P=0.01$ \\
\hline Num $_{S V}>50 \mathrm{~nm}$ from ribbon (EM) & $5.5 \pm 4.2(n=27)$ & $0.8 \pm 1.0(n=34)$ & $P<0.001$ \\
\hline SV diameter (EM, CF) & $41.4 \pm 5.7 \mathrm{~nm}(n=411 \mathrm{SVs})$ & $44.6 \pm 5.6 \mathrm{~nm}(n=431 \mathrm{SVs})$ & $P<0.001$ \\
\hline SV diameter (EM, HPF) & - & $48.7 \pm 8.2 \mathrm{~nm}(n=156 \mathrm{SVs})$ & \\
\hline GluR2/3 cluster (STED) 'size' & $884 \pm 15 \mathrm{~nm}(n=16)$ & $751 \pm 11 \mathrm{~nm}(n=19)$ & $P<0.01$ \\
\hline 'Width' & $180 \pm 0.71 \mathrm{~nm}$ & $193 \pm 0.89 \mathrm{~nm}$ & $P=0.39$ \\
\hline Ratio $_{\max / \mathrm{min}}$ & $2.5 \pm 0.9$ & $2.3 \pm 0.7$ & $P=0.45$ \\
\hline Postsynaptic density (EM) & $819 \pm 154 \mathrm{~nm}(n=29)$ & $888 \pm 200 \mathrm{~nm}(n=30)$ & $P=0.74$ \\
\hline
\end{tabular}

EM, electron microscopy; NumsV, number of synaptic vesicles; SV, synaptic vesicle; CF, chemical fixation; HPF, high pressure freeze; 'size' of GluR2/3 clusters, sum of peak-to-peak distance and average FWHM of the two peaks in a fluorescence profile of the cluster

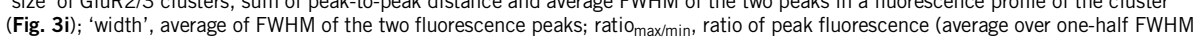
around the peak location) and minimal fluorescence (average over one-half FWHM around the minimum location).

and subsequent freeze substitution (Supplementary Fig. 3 and Supplementary Methods online) because the latter method may better preserve synapse morphology ${ }^{34}$. We did not observe obvious differences in the complement of vesicles; the mean vesicle diameter estimate in the chemically fixed tissue was smaller by less than 10\% (Fig. 3a,b; Table 1). Therefore, we used chemical fixation for further analyses. We did not detect significant differences in ribbon size and shape (short and long axes, cross-sectional area), nor in the length of the postsynaptic density (PSD) between midcochlear (Fig. 3a,b) and apical (Fig. 3c) IHC synapses (Table 1). We quantified the abundance of synaptic vesicles at the active zone for ribbon-associated $(<50 \mathrm{~nm}$ from ribbon) and unassociated ( $>50 \mathrm{~nm}$ from ribbon) vesicles. There were slightly more ribbon-associated vesicles and substantially more unassociated ones in apical synapses compared to midcochlear ones (Table 1).

Optical microscopy of immunolabeled subcellular structures enables high throughput analysis, providing a robust basis for statistical comparison. IHC ribbons, $\mathrm{Ca}^{2+}$ channel clusters and aspects of postsynaptic AMPA receptor clusters (see below) are at or below the resolution limit of confocal microscopy $(\sim 250 \mathrm{~nm})$. Therefore, we used high-resolution 4Pi microscopy (one-dimensional axial resolution $\sim 100 \mathrm{~nm}$; Supplementary Fig. 4 online) and STED microscopy (two-dimensional lateral resolution $\sim 50-150 \mathrm{~nm}$, Fig. 3; threedimensional spherical resolution $\sim 150 \mathrm{~nm}$, Supplementary Movie 1 online) in addition to confocal microscopy to compare those structures between apical and midcochlear synapses. Note that these measurements report apparent rather than absolute object size because of immunolabeling and fluorescence imaging, and thereby overestimate true object size. However, this does not impede the comparison between synapses of different tonotopic regions or sectors of IHCs.

To study ribbons, we analyzed 4Pi images stacks as described previously ${ }^{6}$. The analysis revealed overlapping distributions of apparent axial diameter of ribbons between apical and midcochlear IHCs, indicating similar ribbon size and shape (Supplementary Fig. 4). We fitted gaussian functions to the distributions and found that the means and s.d. values (for apical, $323 \pm 57 \mathrm{~nm}, n=193$ ribbons; for midcochlear, $324 \pm 62 \mathrm{~nm}, n=168$ ribbons) were indistinguishable. Compared to confocal microscopy implemented on the same microscope, STED yielded superior resolution (Fig. 3d,f; lateral dimensions of point spread function (PSF), $\sim 150 \times 150 \mathrm{~nm}$; axial dimension, $\sim 500 \mathrm{~nm}$ at the position of the synapses in the tissue for Fig. $\mathbf{3 d}-\mathbf{g}$ ). The resolution of the STED microscope decreases with the depth of penetration into the tissue and was controlled by measuring the PSF with $100-\mathrm{nm}$ fluorescent beads in front and behind the sample (Fig. 3e,g; Supplementary Fig. 4). The short and long axes of fluorescence spots in the STED sections were approximated as the full widths at half maximum (FWHM) of two orthogonal gaussian functions (Fig. 3e,g). They were slightly but significantly larger for apical ribbons (Table 1 and Supplementary Fig. 4). On average, the mean apparent axes ((short + long)/2) differed by $25 \mathrm{~nm}$ between the two locations. The ratios of long to short apparent axes were statistically indistinguishable (apical, $1.27 \pm 0.16$, $n=144$; midcochlear, $1.31 \pm 0.19, n=$ 136; $P=0.08)$ and were consistent with an ellipsoid ribbon structure. $\mathrm{Ca}_{\mathrm{V}} 1.3 \mathrm{Ca}^{2+}$ channels cluster at active zones of hair cells ${ }^{11,35}$. Using STED microscopy, we studied size and shape of immunolabeled $\mathrm{Ca}_{\mathrm{V}} 1.3$ clusters in IHCs at the two different tonotopic positions (Fig. 3f,g). The size of the observed fluorescent spots (two-dimensional FWHM) ranged between $140 \mathrm{~nm}$ and $650 \mathrm{~nm}$; hence, one of the object axes was typically above the resolution limit of this STED microscope but in many cases too small for estimation by confocal microscopy. Nearby $\mathrm{Ca}^{2+}$ channel clusters (Fig. 3f, right panel), which are readily resolved by STED microscopy but not discernable by confocal microscopy, existed rarely ( $<10 \%$ of all analyzed synapses). The average size and shape of the synaptic $\mathrm{Ca}_{V} 1.3$ clusters were similar for apical and midcochlear IHCs (Fig. 3g, Table 1 and Supplementary Fig. 5 online) (mean apparent axis: apical $278 \pm 71 \mathrm{~nm}, n=84$; midcochlear $264 \pm 69$ $\mathrm{nm}, n=82, P=0.10$; axis ratio: apical $1.45 \pm 0.30$; midcochlear $1.56 \pm$ $0.42 ; P=0.06)$. We modeled the two-dimensional projection of randomly oriented objects (after convolution with the point spread function of the STED microscope; Supplementary Fig. 5) because the real three-dimensional shape of the clusters cannot be readily deduced from the data. We simulated variably sized objects with several geometric shapes aiming to match the experimentally observed distributions of short and long axes (see Supplementary Fig. 5). The data could be reasonably well described by assuming a flat, oblate ellipsoid with diameter $420 \pm 130 \mathrm{~nm}$, short axis below the resolution limit (Fig. 3g). We cannot exclude the existence of subclusters in mice, as proposed for frog hair cells ${ }^{35}$, but, if present, those must be separated by less than $150 \mathrm{~nm}$.

AMPA receptor clusters, detected as GluR2/3 immunofluorescent spots, showed a ring-like shape when oriented in parallel with the $x y$ plane $($ Fig. $3 \mathbf{h}-\mathbf{j})$, indicating a gradient of receptor density in the plane of the PSD with an off-center maximum. The ring-like fluorescence pattern was confirmed by high-resolution three-dimensional STED microscopy (Supplementary Movie 1). We cannot entirely rule out lower accessibility to antibody labeling of AMPA receptors in the center of the synapse. However, we consider this highly unlikely, as a similar gradient of receptor density was found in a previous immunoelectron microscopy study of rat IHC afferent synapses ${ }^{12}$, where antigen accessibility should not be a concern owing to postembedding immunogold labeling of AMPA receptors in ultrathin sections. En face views of AMPA 

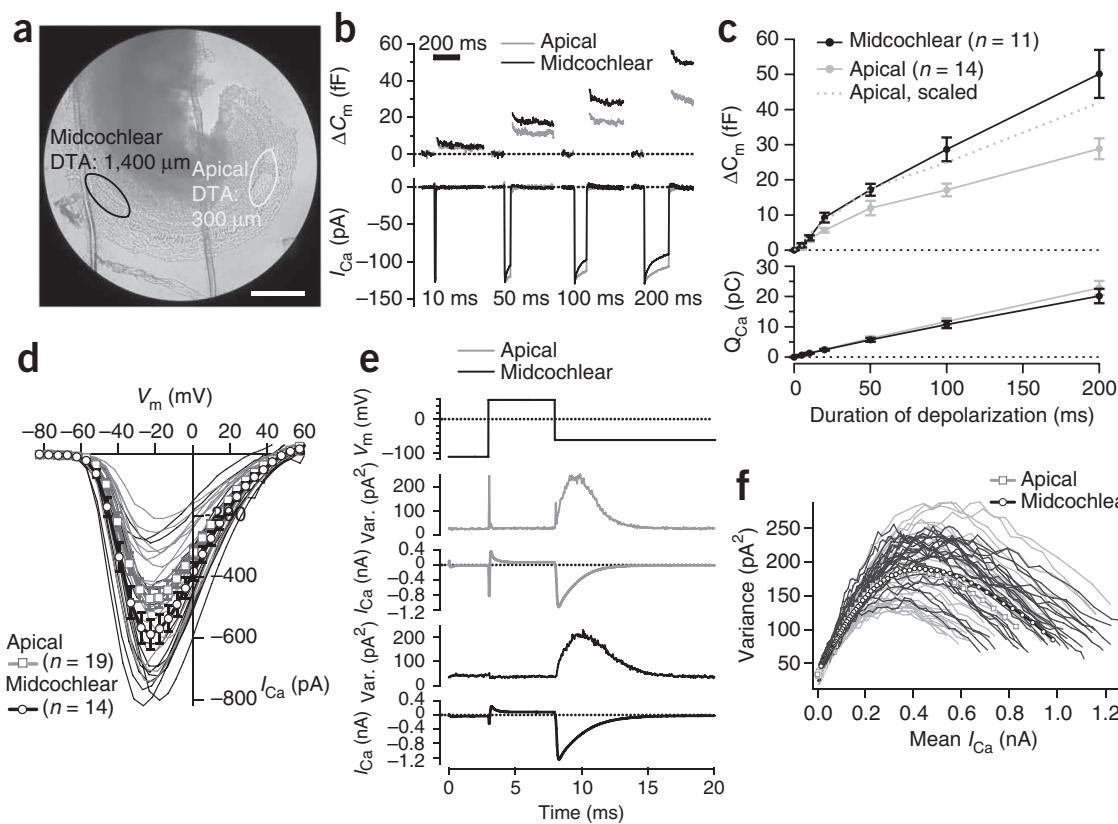

$\infty-(n=14)$

Figure 4 Exocytosis scales with the number of afferent synapses per IHC. (a) Distance-to-apex (DTA) measurement of representative tonotopic regions used for patch-clamp recordings, identified by locally removed rows of outer hair cells and pillar cells (ellipses). Scale bar, $200 \mu \mathrm{m}$. (b) Average membrane capacitance $\left(C_{\mathrm{m}}\right)$ traces (baseline subtracted) and calcium current $\left(I_{\mathrm{Ca}}\right)$ of apical $(n=11$; gray) and midcochlear ( $n=14$; black) IHCs in response to 10-, 50-, 100- and 200-ms depolarizations. (c) Mean exocytic $\Delta C_{m}$ (top) and $\mathrm{Ca}^{2+}$ current integrals (bottom) of the same cells as a function of stimulus duration. Scaled apical (dotted line) is $C_{\mathrm{m}}$ of apical IHCs multiplied by 1.44 (ratio of the number of synapses for midcochlear and apical IHCs). (d) Average current-voltage relationships (thick traces with symbols) of apical ( $n=19$, thin gray traces) and midcochlear ( $n=14$, thin black traces) IHCs in augmenting conditions (10 mM extracellular [Ca $\left.{ }^{2+}\right], 5 \mu \mathrm{M}$ BayK8644). (e,f) Analysis of $\mathrm{Ca}^{2+}$ tail current fluctuations in augmenting conditions: (e) Pulse protocol and typical examples of current ensemble variance and mean of an apical and a midcochlear IHC, respectively. (f) Variance-mean relationships of all apical and midcochlear IHCs (light and dark thin traces, respectively) and grand mean for both cell populations (thick traces with symbols). Error bars, s.e.m.

receptor clusters were analyzed by fitting the STED (Fig. 3i) fluorescence profiles with a sum of two gaussians. As expected, the limited resolution of confocal microscopy led to an overestimation of the peak width (confocal $\sim 350 \mathrm{~nm}$; STED $\sim 180 \mathrm{~nm}$ (lateral resolution $<80 \mathrm{~nm}$ for this microscope)), and thus we based further analysis solely on STED microscopy. Whereas the width of the peaks was statistically indistinguishable between apical and midcochlear clusters, we found slightly larger peak-to-peak distances (apical $683 \pm 84 \mathrm{~nm}, n=16$; midcochlear $562 \pm 85 \mathrm{~nm}, n=19$ ) and total sizes for clusters of apical synapses (Table 1). The outer diameter of the cluster roughly matched the length of the PSD as measured by electron microscopy (Table 1), indicating that AMPA receptors populate most of the PSD. On average, the fluorescence peaks were about twofold brighter than the center, for both apical and midcochlear AMPA receptor clusters (Table 1).

\section{Tonotopy of IHC presynaptic physiology}

Recent studies have used patch-clamp recordings of $\mathrm{Ca}^{2+}$ currents and membrane capacitance changes $\left(\Delta C_{\mathrm{m}}\right)$ to study the presynaptic properties of hair cells of various species (for review, see ref. 3 ). It remains to be clarified whether this technique reports exclusively synaptic $\mathrm{Ca}^{2+}$ current and exocytosis in IHCs ${ }^{35-37}$ or also a significant extrasynaptic fraction of channels and/or vesicles $5,6,11,38,39$. We also wondered whether, on top of the number of synapses, their function may also vary along the cochlea's tonotopic axis ${ }^{15,16,30,31,37}$. We first studied whole-cell $\mathrm{Ca}^{2+}$ currents and exocytic membrane capacitance changes
$\left(\Delta C_{\mathrm{m}}\right)$ in IHCs from those apical and midcochlear regions (Fig. 4a) that had also been morphologically investigated (Figs. 1-3). IHCs at these two locations had $14.0 \pm 0.3$ (midcochlear) and $9.7 \pm 0.7$ (apical) synapses, respectively, and, as judged from their resting membrane capacitance (midcochlear IHCs $8.7 \pm 1.1 \mathrm{pF}, n=11$; versus apical IHCs 7.7 $\pm 0.7 \mathrm{pF}, n=14$ ), they differed slightly in size. We recorded their $\mathrm{Ca}^{2+}$ currents and membrane capacitance increments in the perforated-patch configuration in response to step depolarizations to $-14 \mathrm{mV}$ (Fig. 4b,c). For most stimuli, we identified larger exocytic $\Delta C_{\mathrm{m}}$ for midcochlear IHCs. Exocytosis of midcochlear and apical IHCs could be roughly matched when scaling the responses of apical IHCs by the ratio of the number of synapses (1.44; Fig. 4c).

Midcochlear IHCs tended to show larger peak $\mathrm{Ca}^{2+}$ currents (ratio of peak currents; under non-augmenting conditions $(2 \mathrm{mM}$ $\left[\mathrm{Ca}^{2+}\right]$, perforated-patch): 1.1, augmenting conditions $\left(10 \mathrm{mM}\right.$ extracellular $\left[\mathrm{Ca}^{2+}\right]$, $5 \mu \mathrm{M}$ BayK8644, whole-cell): 1.2, Fig. 4d and Supplementary Table 2 online). The difference in $\mathrm{Ca}^{2+}$ current integrals (Fig. 4c) did not reach statistical significance, probably because of the more pronounced $\mathrm{Ca}^{2+}$ current inactivation in midcochlear IHCs (Fig. 4b). Neither were voltage dependence and kinetics of $\mathrm{Ca}^{2+}$ current activation different (Supplementary Fig. 6 online). Assuming an exclusively synaptic localization of $\mathrm{Ca}^{2+}$ channels and given the roughly comparable size of synaptic clusters of $\mathrm{Ca}^{2+}$ channels (Fig. 3), one would have expected the peak $\mathrm{Ca}^{2+}$ currents in midcochlear and apical IHCs to scale with the number of synapses, as seen for exocytosis. The observed discrepancy could be due to differences in channel open probability or single-channel current among IHCs at the two positions. When analyzing fluctuations among repetitively evoked $\mathrm{Ca}^{2+}$ tail currents under augmenting conditions (Fig. 4e,f), we found more $\mathrm{Ca}^{2+}$ channels (1.34:1, midcochlear/apical), a slightly higher open probability and a somewhat smaller single-channel current in the midcochlear cells (see Supplementary Table 2).

Conclusions from whole-cell recordings on synaptic $\mathrm{Ca}^{2+}$ channels would be confounded by the presence of extrasynaptic $\mathrm{Ca}^{2+}$ channels. To directly compare synaptic $\mathrm{Ca}^{2+}$ signaling at different tonotopic places, we used fast confocal imaging of IHCs loaded with Fluo-5N. Depolarization caused the rapid appearance of spatially restricted fluorescence hotspots in the basolateral compartment of IHCs from hearing mice (Fig. 5a) that were mediated by voltage-gated $\mathrm{Ca}^{2+}$ influx and localized with ribbons ${ }^{40}$, as previously described in nonmammalian hair cells ${ }^{36,41,42}$. Once such $\mathrm{Ca}^{2+}$ microdomains were identified in confocal sections, we used spot detection (continuous read-out of fluorescence from the maximum-intensity location inside the $\mathrm{Ca}^{2+}$ microdomain (Fig. 5b,c) and perpendicular line scans (Fig. 5d,e) to study the kinetics (Fig. 5b), voltage dependence (Fig. 5c) and FWHM (Fig. 5e) of these synaptic $\mathrm{Ca}^{2+}$ signals during 20-ms depolarizations). On average, these properties were indistinguishable between apical and midcochlear IHCs (Table 2). 

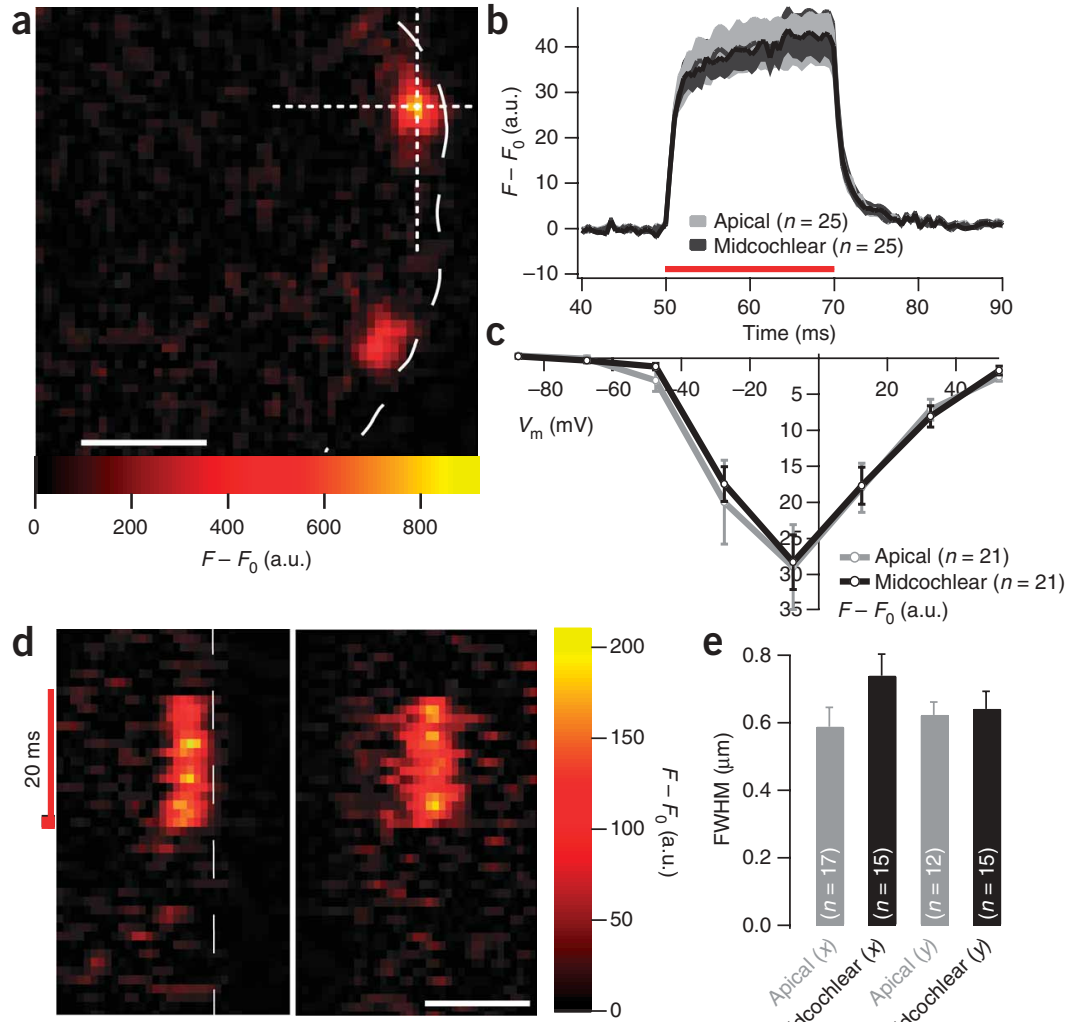

e

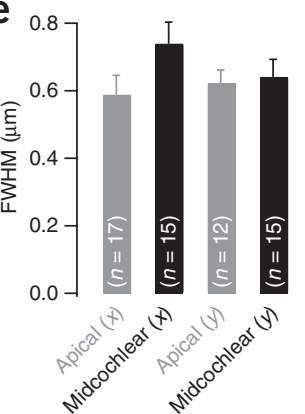

Figure 5 Synaptic $\mathrm{Ca}^{2+}$ signals are comparable at different tonotopic locations. (a) $x y$ scan reveals hotspots of Fluo- $5 \mathrm{~N}$ fluorescence in the base of a P14 IHC (loaded with $400 \mu \mathrm{M}$ Fluo- $5 \mathrm{~N}$ and $2 \mathrm{mM}$ EGTA; dotted line, IHC border) during membrane depolarization to $-7 \mathrm{mV}$ (red bar, time of depolarization). Resting fluorescence $\left(F_{0}\right)$ was subtracted. Scale bar, $2 \mu \mathrm{m}$; a.u., arbitrary fluorescence intensity units. (b) Fluorescence time courses for $\mathrm{Ca}^{2+}$ microdomains in apical (gray) and midcochlear (black) IHCs. Data were obtained at high temporal resolution at the brightest pixel of the hotspot (spot detection; see Methods) (dotted crosshairs in a), on which the laser beam was parked. Red bar, depolarization to $-7 \mathrm{mV}$. (c) $\mathrm{Ca}^{2+}$ microdomain amplitude as a function of membrane potential. The average $\Delta F$ amplitude over the last $15 \mathrm{~ms}$ of the respective spot detection response to $20 \mathrm{~ms}$ depolarizations is plotted. (d) Line scan. Dashed line in left panel, $\mathrm{IHC}$ border. (e) Spatial extent of $\mathrm{Ca}^{2+}$ microdomains. FWHM values were obtained by fits of gaussian functions to time-averaged (20 ms) line scans (d). Error bars, s.e.m.

and starting $x y$-position in repetitive $x y$-scans, followed by (ii) selection of the brightest pixel identified during lateral spot displacement along one dimension (from the starting position). Moreover, we obtained a second estimate by fitting gaussian functions to the line scans, and we found the variability comparable (Supplementary Table 3). The scatter of fluorescence amplitude (see Methods) for 65 individual synapses in 20 apical and midcochlear IHCs responding to $20 \mathrm{~ms}$ depolarizations to $-7 \mathrm{mV}$ (Fig. 6a) had a coefficient of variation $(\mathrm{CV})$ of 0.63 . Similar variability was also found for the background-normalized data $\left(\Delta F / F_{0}, \mathrm{CV}=0.61\right)$, arguing against a contribution of variation in dye concentration.

Even synapses of an individual IHC differed up to tenfold in their fluorescence amplitude (Fig. 6b; maximum/minimum $\Delta F$ on average, 4.5 -fold; average $\mathrm{CV}=0.57$ for seven apical IHCs). The variance among the fluorescence changes was not systematic with time (Fig. 6b), arguing against $\mathrm{Ca}^{2+}$ current run-down, indicator bleaching, or buffer saturation being sizeable contributors. The trial-to-trial variability of $\Delta F$ of the same $\mathrm{Ca}^{2+}$ microdomain

Peak $\Delta F\left(F-F_{0}\right.$, a.u. $)$

$A_{\text {onset,fast }}$ (ms)

$A_{\text {onset,slow (ms) }}$

$A_{\text {onset,fast }} / A_{\text {onset,slow }}$

$A_{\text {decay,fast }}(\mathrm{ms})$

$A_{\text {decay,slow }}(\mathrm{ms})$

$A_{\text {decay,fast }} / A_{\text {decay,slow }}$

$$
\begin{aligned}
39.1 & \pm 5.3(n=25) \\
1.0 & \pm 0.1(n=25 / 25) \\
17 & \pm 8(n=8 / 25) \\
2.3 & \pm 0.7(n=8 / 25) \\
1.1 & \pm 0.1(n=25 / 25) \\
8 & \pm 1(n=13 / 25) \\
5.1 & \pm 0.8(n=13 / 25)
\end{aligned}
$$

Midcochlear (mean \pm s.e.m.)

$P$-value

$V_{1 / 2}(\mathrm{mV})$

$-26.1 \pm 3.8(n=13)$

$7.3 \pm 1.4(n=13)$

$$
\begin{aligned}
39.3 & \pm 4.5(n=25) \\
1.0 & \pm 0.1(n=25 / 25) \\
60 & \pm 38(n=12 / 25) \\
1.5 & \pm 0.4(n=12 / 25) \\
1.0 & \pm 0.1(n=25 / 25) \\
28 & \pm 10(n=13 / 25) \\
3.9 & \pm 0.7(n=13 / 25) \\
-26.1 & \pm 2.0(n=13) \\
6.3 & \pm 0.6(n=13)
\end{aligned}
$$

$P=0.97$

$P=0.16$

$P=0.16$ was small $(\mathrm{CV}=0.09)$. We found only weak, although significant, correlations

0.16 between fast-onset kinetics and fluores-

$P=0.24$ cence amplitude (Fig. 6c; linear correlation

$P=0.11$ coefficient $\left.P_{r}=0.28, P<0.05\right)$, and

$P=0.27 \quad$ FWHM of $\mathrm{Ca}^{2+}$ microdomains and their

$P=0.28$

$P=0.65$

Line scan

$x$ FWHM $(\mu \mathrm{m})$

$0.6 \pm 0.1(n=17)$

$0.7 \pm 0.1(n=15)$

$P=0.09$ $y$ FWHM ( $\mu \mathrm{m})$

$0.6 \pm 0.1(n=12)$

$P=0.35$

Exponential fitting was used to estimate the on- and off-kinetics of the synaptic $\mathrm{Ca}^{2+}$ microdomain signals. A double-exponential fit was accepted if the time constants differed by at least a factor of 3 and if both components were sizeable. $A$, amplitude of the fluorescence component indicated in the subscript; $V_{1 / 2}$, voltage of half-maximal fluorescence increase; slope, steepness of the voltage-dependent increase of the fluorescence change. $V_{1 / 2}$ and slope were obtained by fitting Boltzmann functions to the fluorescence-voltage relationships (Fig. 5c). FWHM, full width at half-maximum of the time-averaged $\mathrm{Ca}^{2+}$ microdomain fluorescence. $x$ and $y$ designate the directions of the orthogonal line scans; a.u., arbitrary fluorescence intensity units. fluorescence amplitude (Fig. 6d; $P_{r}=$ $-0.23, P<0.05)$. Taken together, these correlations argue against a substantial contribution of defocus from the $\mathrm{Ca}^{2+}$ microdomain's center to the observed variance. We therefore interpret these differences to mostly reflect genuine variance among $\mathrm{Ca}^{2+}$ signals of the individual synapses.

Next, we asked whether active zones with different properties segregated along the perimeter of the IHC, as previously 
a
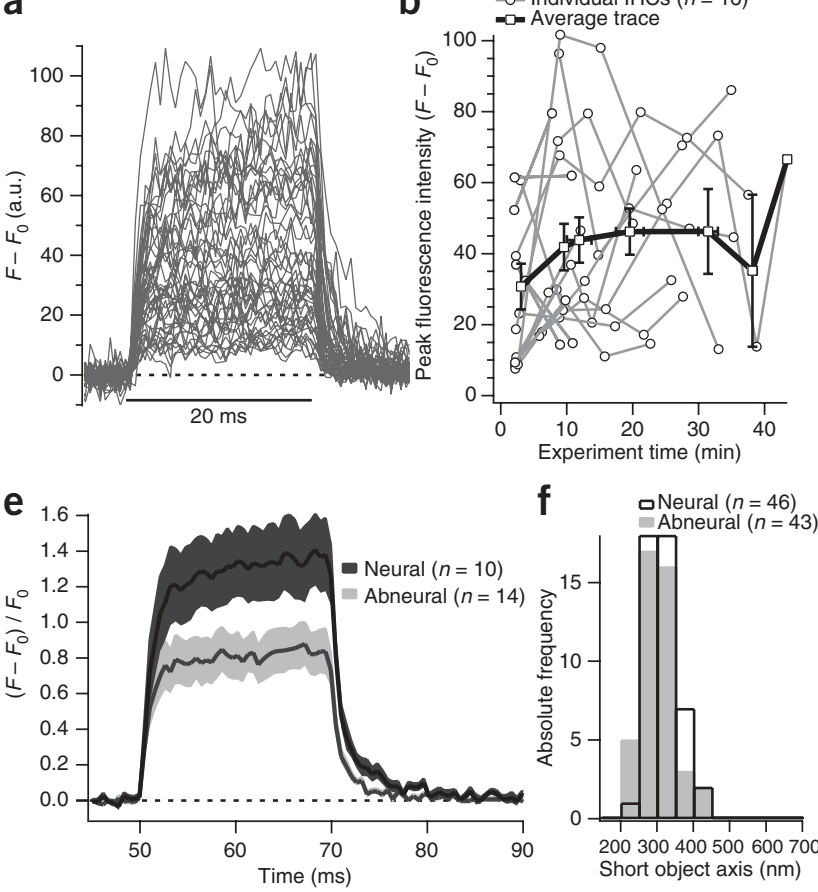

suggested in the $\mathrm{cat}^{24}$. We used confocal $\mathrm{Ca}^{2+}$ imaging and STED microscopy of immunolabeled ribbons in the neural and abneural $60^{\circ}$ sectors to test for potential functional and morphological differences of the respective synapses. Abneural synapses on average showed a smaller fluorescence increment (Fig. 6e; $\Delta F / F_{0}$ neural/abneural ratio, 1.6). The distributions of short (Fig. 6f) and long axes of ribbons (Fig. 6g) were indistinguishable, suggesting that the average ribbon size did not differ among neural and abneural synapses in mouse IHCs.

\section{DISCUSSION}

Our findings demonstrated that tonotopic variations in the sensitivity of the mammalian cochlea are paralleled by the density of its afferent innervation. We present nanoscale estimates of the size of ribbons, $\mathrm{Ca}^{2+}$ channel and AMPA receptor clusters at the IHC synapse for two different tonotopic regions. Although substantially changing in number, IHC synapses in these cochlear regions differ only slightly in structure. Exocytosis of IHCs scaled with the number of synapses, which, together with the comparable average properties of presynaptic $\mathrm{Ca}^{2+}$ signals, suggests a fairly uniform average synapse function in these two regions and also, presumably, further along the cochlea's tonotopic axis. However, $\mathrm{Ca}^{2+}$ signals showed substantial heterogeneity among synapses of an individual IHC, providing a potential presynaptic substrate for the divergent spiking properties of the SGNs that are driven by a given IHC.

Quantitative light microscopy of whole-mounted organs of Corti allowed assembly of continuous synaptic cochleograms for mouse (high-frequency hearing) and gerbil (low-frequency hearing) with a sample size and frequency resolution exceeding those of previous electron microscopy studies ${ }^{17,20,21,43}$. Synaptic cochleograms were well described by quadratic functions, providing a simple and useful tool for future studies on cochlear neurotransmission. The shape of the mouse synaptic cochleogram roughly followed that of the mouse behavioral audiogram ${ }^{44}$, that of the neural population audiogram (for example, Fig. 1) and that of the distortion product otoacoustic emission audiogram (for example, ref. 45). We interpret these findings as better neural sampling from the most sensitive cochlear regions. In
C

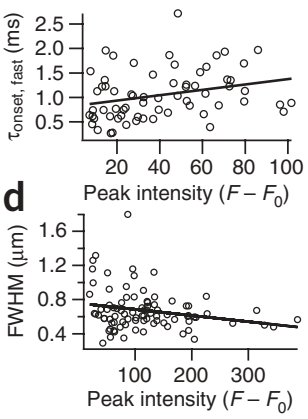

g

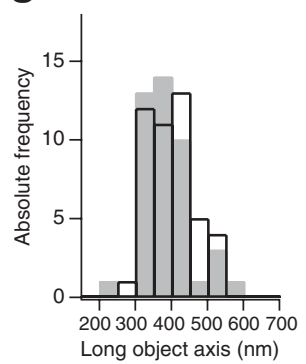

Figure 6 Intracellular variability of synaptic $\mathrm{Ca}$ microdomains. (a) Time course of fluorescence at the center of $\mathrm{Ca}^{2+}$ microdomains during 20-ms depolarization to peak $\mathrm{Ca}^{2+}$ current potential ( $-7 \mathrm{mV}$, as described in Fig. $5 \mathrm{c} ; n=65 \mathrm{Ca}^{2+}$ microdomains in $20 \mathrm{IHCs}$ ); a.u., arbitrary fluorescence intensity units. (b) $\mathrm{Ca}^{2+}$ microdomain amplitude within individual IHCs as a function of time of acquisition. (c,d) $\mathrm{Ca}^{2+}$ microdomain amplitude ( $\Delta F$ amplitude over the last $15 \mathrm{~ms}$ of data in a) correlates weakly with fast onset kinetics (c, linear correlation coefficient $\left.P_{r}=0.28, n=65\right)$ and with its FWHM (d; $\left.P_{r}=0.23, n=87\right) . \Delta F$ amplitude and FWHM were obtained by fitting gaussian functions to time-averaged line scans. (e) Differences in average peak $\mathrm{Ca}^{2+}$ microdomain amplitude between neural and abneural synapses $(P<0.05$, for peak amplitude). Gray bands, s.e.m. $(\mathbf{f}, \mathbf{g})$ Overlapping distributions of short and long ribbon axes of neural and abneural synapses of apical IHCs (distance to apex, 100-300 $\mu \mathrm{m}$ ) as estimated by STED microscopy.

the mouse, this region encodes the range of $\sim 10-20 \mathrm{kHz}$, which is important, for example, for the perception of wriggling calls of mouse pups ${ }^{46}$. Each SGN conveys information transmitted by one hair cell synapse to several neurons of the cochlear nucleus, which in turn integrate information from several SGNs. The reliability and acuity of afferent information provided by a region of the cochlea will increase with the number of innervating SGNs.

Here we took advantage of the tonotopic gradient of synapse number but uniform average presynaptic morphology in the mouse cochlea to ask whether whole-cell $\mathrm{Ca}^{2+}$ current and exocytic $\Delta C_{\mathrm{m}}$ scaled with the number of ribbon synapses, as would be expected if both occurred exclusively at the synapse. This was indeed found to be the case for exocytosis, as previously described for turtle hair cells ${ }^{37}$. Although we cannot exclude some contribution of vesicles fusing outside the active zone to exocytosis seen during prolonged stimulation ${ }^{3,47}$, we suppose that much of this sustained release occurs at the synapse. This view is supported by paired pre- and postsynaptic recordings from rat IHC synapses ${ }^{8}$. These data revealed AMPA receptor current integrals that were compatible with the notion of sustained exocytosis-as reported by capacitance measurements-reflecting synaptic release.

Our STED data suggest that, at the IHC synapse, AMPA receptors have a peripheral, 'ring-like' density maximum, which correlates with electron microscopy data on the rat cochlea ${ }^{12}$. The observed receptor distribution seems well suited for the efficient detection of glutamate release, in particular if this release occurs preferentially at the circumference of the ribbon ${ }^{35}$. It deviates from the uniform receptor distribution of glutamatergic CNS synapses suggested by immunoelectron microscopy (ref. 12; see review in ref. 48).

The $\mathrm{Ca}^{2+}$ current integral was not significantly different between apical and midcochlear IHCs. In part, this can be attributed to the stronger $\mathrm{Ca}^{2+}$ current inactivation in IHCs in the higher frequency region, which is consistent with findings in the gerbil ${ }^{49}$. Still, even the initial $\mathrm{Ca}^{2+}$ current was somewhat less correlated to synapse number than exocytosis. Similar findings were obtained in the turtle cochlea, where low-frequency synapses were assumed to mediate larger $\mathrm{Ca}^{2+}$ influx ${ }^{37}$. Unlike that study, we conclude, based on analysis of $\mathrm{Ca}^{2+}$ current, synaptic $\mathrm{Ca}^{2+}$ signals and $\mathrm{Ca}^{2+}$ channel cluster size that, except for inactivation, $\mathrm{Ca}^{2+}$ signaling of the average mouse IHC synapse is structurally and functionally similar 
in the two frequency regions. The existence of extrasynaptic $\mathrm{Ca}^{2+}$ channels, as suggested by single channel recordings from bullfrog hair cells ${ }^{39}$, is likely to explain the small remaining mismatch between the scaling of number of $\mathrm{Ca}^{2+}$ channels (1.34) and number of synapses (1.44).

Using various approaches, we compared, at two different frequency regions, key morphological determinants of IHC synaptic transmission, including synaptic ribbons and vesicle complement, synaptic $\mathrm{Ca}^{2+}$ channel clusters and postsynaptic density. We found only subtle differences (Fig. 3, Table 1), all indicative of slightly larger IHC synapses in the apex of the cochlea. Testing the functional relevance of these tonotopic differences requires more sensitive detection, such as in paired pre- and postsynaptic recordings ${ }^{8}$.

However, we found considerable variability for all of the investigated morphological and functional synapse parameters (Fig. 3, Tables 1 and 2, Supplementary Table 3 and Supplementary Fig. 4 and 5). Although this limits detection of small systematic changes along the tonotopic axis, it probably relates to the important question in auditory research of the mechanisms behind the different functional properties of SGNs. Pioneering work primarily in the cat cochlea has identified morphological correlates for high and low spontaneous firing rate SGNs (for example, active zone morphology, abneural versus neural insertion; diameter and mitochondrial content of the peripheral axon) ${ }^{14,22,24,43}$. In the present study, we observed slightly lower synaptic $\mathrm{Ca}^{2+}$ signals in the abneural sector of IHCs and found ribbon sizes to be comparable for neural and abneural synapses. These findings seem hard to reconcile with a systematically higher activity of abneural synapses in mouse IHCs, for which one might have expected larger ribbons providing more releasable vesicles ${ }^{3}$ and more $\mathrm{Ca}^{2+}$ channels.

Nevertheless, our experiments revealed substantial heterogeneity between $\mathrm{Ca}^{2+}$ signals at active zones within individual IHCs (Fig. 6b and Supplementary Table 3 ). Furthermore, we found a large variation of $\mathrm{Ca}_{\mathrm{V}} 1.3$ channel cluster size $(\mathrm{CV}=0.3)$, and, assuming a constant channel density in the cluster, we propose that differences in channel number contribute to this heterogeneity. Placement of synapses with different properties seems rather random in mouse IHC. Future studies combining imaging of synaptic $\mathrm{Ca}^{2+}$ signals with readouts of transmitter release and/or postsynaptic response ${ }^{7,8}$ will test how this translates into differences of transmitter release among the synapses of an individual mouse IHC.

\section{METHODS}

Animals. C57BL/6 and NMRI (Naval Medical Research Institute) mice aged 2-4 weeks and 4-week-old gerbils were used for experiments. Animal experiments complied with national animal care guidelines and were approved by the University of Göttingen Board for animal welfare and the animal welfare office of the state of Lower Saxony.

\section{Auditory brainstem responses. See Supplementary Methods.}

Patch-clamp and confocal $\mathrm{Ca}^{2+}$ imaging. IHCs from apical coils of freshly dissected organs of Corti from NMRI and C57BL/6 mice (P14-18) were patchclamped as described ${ }^{5}$. The pipette solution for perforated-patch recordings contained (in mM) 140 cesium gluconate, 13 tetraethylammonium (TEA)-Cl, $10 \mathrm{CsOH}$-HEPES buffer, $1 \mathrm{MgCl}_{2}$, and $250 \mu \mathrm{g} / \mathrm{ml}$ amphotericin $\mathrm{B}, \mathrm{pH}$ 7.2. The pipette solution for whole cell recordings contained (in $\mathrm{mM}$ ) 135 cesium glutamate, 13 TEA-Cl, 20 CsOH-HEPES, $1 \mathrm{MgCl}_{2}$, 2 Mg-ATP, 0.3 Na-GTP, 2 EGTA (10 for biophysical analysis of $\mathrm{Ca}^{2+}$ currents), 0.4 Fluo-5N (penta- $\mathrm{K}^{+}$ salt, Invitrogen; for confocal imaging), $\mathrm{pH}$ 7.0. The extracellular solution contained (in mM) $105 \mathrm{NaCl}, 35 \mathrm{TEA}-\mathrm{Cl}, 2.8 \mathrm{KCl}, 2 \mathrm{CaCl}_{2}$ (10 for fluctuation analysis, 5 for confocal imaging, balanced by $\mathrm{NaCl}$ ), $1 \mathrm{MgCl}_{2}, 10 \mathrm{NaOH}-$ HEPES, 10 D-glucose and 0.005 BayK8644 (Tocris, for fluctuation analysis), pH 7.2 (7.3 for whole-cell recordings). EPC-9 amplifiers controlled by Pulse or
Patchmaster software (HEKA Elektronik) were used for measurements. All voltages were corrected for liquid-junction potentials. Currents were low-pass filtered at $14 \mathrm{kHz}$ and $2 \mathrm{kHz}$ and sampled at $100 \mathrm{kHz}$ and $10 \mathrm{kHz}$ for whole-cell recordings and perforated-patch recordings, respectively. Cells that showed a holding current exceeding $-50 \mathrm{pA}$ were discarded from analysis. $\mathrm{Ca}^{2+}$ currents were further isolated using a $\mathrm{P} / \mathrm{n}$ protocol. Series resistance was required to be below $30 \mathrm{M} \Omega$ for perforated-patch experiments and averaged 10.0 $\pm 0.5 \mathrm{M} \Omega$ $(n=59)$ in the fluctuation analysis experiments. Fluctuation analysis was performed similarly to that previously described ${ }^{11}$. To account for channel gating-related and filter-induced correlations between neighboring currentvariance data points, we fitted the variance-over-mean data by an estimated generalized least-squares method (Supplementary Table 2). The first $600 \mu$ s of the tail current routinely were discarded. To avoid errors introduced by remaining, uncancelled capacitive transients, we subtracted a scaled version of the transient that was evident at the beginning of the depolarization step (see Fig. 4e) from the mean current trace (after calculation of the ensemble variance). Membrane capacitance increments $\left(\Delta C_{\mathrm{m}}\right)$ were measured as previously described ${ }^{5}$. Interstimulus periods were $2-3 \mathrm{~s}$ between sweeps, 1-2 min between ensembles for confocal imaging, and 30-70 s for exocytosis measurements.

Confocal $\mathrm{Ca}^{2+}$ imaging was performed as described ${ }^{40}$, using a Fluoview 300 confocal scanner mounted on an upright microscope (BX50WI) equipped with a 0.9 numerical aperture (NA), $\times 60$, water immersion objective (all Olympus) and a 50-mW, 488-nm, solid-state laser (Cyan, Newport-Spectraphysics). Fluorescent hotspots were identified in $x y$-scans during 200-ms depolarizations $(0.5 \%$ of maximum laser intensity) and further characterized using spot detection ('point scan' mode of the confocal scanner, temporally averaged to yield an effective sampling rate of $1.85 \mathrm{kHz}, 0.05 \%$ of maximum laser intensity) and line scans (at a rate of $0.74 \mathrm{kHz}, 0.25 \%$ of maximum laser intensity). Spot detection measurements and line scans were repeated 5 and 10 times, respectively, to improve signal-to-noise ratio. Peak $\Delta F$ estimates of spot detection measurements were obtained after repetitive boxcar smoothing (2-ms box). On average, we characterized 3.1 and 1.7 spots per IHC for apical-basal and neural-abneural comparisons, respectively. The average $\mathrm{Ca}^{2+}$ current rundown at the end of the experiment was $30 \%$ of the maximum current. For investigating intracellular differences, $\mathrm{Ca}^{2+}$ microdomain characterization was followed by acquisition of a $z$-stack of the indicator-filled cell so that its location could be retrieved.

Immunohistochemistry. Immunostaining was performed as previously described $^{6}$. Briefly, the freshly dissected apical cochlear turns were fixed with $4 \%$ paraformaldehyde for $1 \mathrm{~h}$ on ice, with $2 \%$ formaldehyde for $10 \mathrm{~min}$ at $20-22{ }^{\circ} \mathrm{C}$ (for staining with antibody to GluR2/3 (anti-GluR2/3)), or for $25 \mathrm{~min}$ in $99 \%$ methanol at $-20{ }^{\circ} \mathrm{C}$ (for anti-Ca 1.3 ). For harvesting the fulllength organs of Corti, cochleae were fixed by cochlear perfusion with $2 \%$ formaldehyde for $10 \mathrm{~min}$. The following antibodies were used: mouse IgG1 anti-CtBP2 (also recognizing the ribbon protein RIBEYE; BD Biosciences, 1:150), rabbit anti-GluR2/3 (Chemicon, 1:200), rabbit anti-calbindin (Swant, 1:400), rabbit anti-Cav1.3 (Alomone Labs, 1:75) and secondary Alexa Fluor 488- and Alexa Fluor 568-labeled antibodies (Molecular Probes, 1:200) as well as Atto-647N (AttoTech, 1:60 dilution in PBS with addition of $2 \%$ normal goat serum) for STED microscopy. In some experiments, nuclei were stained with Hoechst 34580 (Molecular Probes, 1:1,000).

Confocal, 4Pi and STED microscopy. Confocal morphological images were acquired using a laser scanning confocal microscope (Leica TCS SP5, Leica Microsystems) with 405-nm (diode), 488-nm (argon) and 561-nm (diodepumped solid state) lasers for excitation and a $\times 63$ oil immersion objective $(\mathrm{NA}=1.4)$. Whole-mount preparations of the organ of Corti allowed us to analyze several IHCs in a row, as previously described ${ }^{6}$. For three-dimensional reconstructions of the specimen, $z$-axis stacks of two-dimensional images were taken with a step size of $0.049 \mu \mathrm{m}, 0.2 \mu \mathrm{m}$ or $0.3 \mu \mathrm{m}$. Multifocal $4 \mathrm{Pi}$ microscopy with water immersion lenses (NA 1.2) at a two-photon excitation wavelength of $870 \mathrm{~nm}$ (average power, $1.5 \mathrm{~mW}$ for each of the $4 \mathrm{Pi}$ foci) was performed at a custom microscope as described ${ }^{6,26,27}$. For STED imaging, two different microscopes were used: (i) a Leica TCS STED microscope (Fig. 3d-g) using two pulsed lasers for excitation (diode laser, $635 \mathrm{~nm}$, 
$<90 \mathrm{ps}$ ) and stimulated emission (Ti:sapphire, $750 \mathrm{~nm}, \sim 300 \mathrm{ps}$ ), with both lasers running at a repetition rate of $80 \mathrm{MHz}$ and synchronized to each other to ensure optimal STED efficiency in the focal plane; and (ii) a custom microscope ${ }^{50}$ (Fig. $\left.3 \mathbf{h}-\mathbf{j}\right)$. This microscope used identical lasers but allowed for higher STED powers and therefore exhibited a resolution of $\sim 50 \mathrm{~nm}$ and $\sim 150 \mathrm{~nm}$ for two-dimensional $(x, y)$ and three-dimensional $(x, y, z)$ imaging, respectively. Single confocal and STED images of ribbons and $\mathrm{Ca}^{2+}$ channel clusters were acquired after adjusting the focus of the $\times 100$ oil immersion lens $(\mathrm{NA}=0.7$ or 1.4$)$ to the fluorescence maximum of an object of interest, as found in a $x z$-scan.

\section{Electron microscopy. See Supplementary Methods.}

Data analysis. Data was analyzed using Matlab (MathWorks), Igor Pro (Wavemetrics) and ImageJ software. Figures were assembled for display in Adobe Photoshop and Illustrator software. Mean $\Delta C_{\mathrm{m}}$ and $\mathrm{Ca}^{2+}$ current estimates are grand means calculated from the mean estimates of individual IHCs. Means were expressed \pm s.d. (or s.e.m. when noted). If applicable (that is, as determined by normality of the distribution (Jarque-Bera test) and equal variances between ( $F$-test) the two samples), an unpaired, two-tailed $t$-test was used to compare the two samples. In all other cases, a Mann-WhitneyWilcoxon test was used.

Image analysis. For synaptic cochleograms, CtBP2/RIBEYE immunofluorescence spots in the basolateral portion of IHCs (up to the apical end of the CtBP2-stained nucleus) were counted in $z$-stacks and divided by the number of IHCs (taken as the quantity of nuclei in the field of view). Estimation of the cellular synapse distribution was performed using custom-written MATLAB routines (available at http://www.innerearlab.uni-goettingen.de/) that included (i) alignment of the image stacks with the tonotopic axis, (ii) image segmentation into ribbons (positive, if more than four connected voxels were above threshold) and nuclei, (iii) center of mass calculation for both types of structures (by a three-dimensional gaussian fit in the case of nuclei), (iv) assignment of ribbons to the closest IHC nucleus, (v) alignment of the individual IHC's $z$-axis to a common $z$-axis, (vi) normalization of the cell's $z$-extent (measured from the center of the nucleus to the basal end of the IHC) to the population average and (vii) vector calculation. For Figure $\mathbf{2} \mathbf{b}$, we also normalized the radial extent of the IHCs for improved superposition. The FWHM in confocal and STED images was estimated using gaussian functions (two-dimensional for morphology, one-dimensional for functional imaging). The two-dimensional data in Figure $\mathbf{3} \mathbf{j}$ was linearly deconvolved with a two-dimensional gaussian PSF (FWHM of $70 \mathrm{~nm}$ for the STED and $250 \mathrm{~nm}$ for the confocal).

Note: Supplementary information is available on the Nature Neuroscience website.

\section{ACKNOWLEDGMENTS}

We thank E. Neher, M. Göpfert, R. Nouvian, N. Strenzke, M. Müller and A. Lysakowski for comments on the manuscript; K. Wadel and C. Henrich for participation in an early stage of the project; A. Neef for image analysis support; J. Hegerman and S. Eimer for support with high-pressure rapid freeze and freeze substitution; and C. Rüdiger and M. Köppler for technical assistance. This work was supported by grants from the Deutsche Forschungsgemeinschaft (Center for Molecular Physiology of the Brain; T.M., A.E. and S.W.H), a Lichtenberg Fellowship from the state of Lower Saxony (T.F.), the European Commission (Eurohear, T.M.), the Max-Planck-Society (Tandemproject, T.M.), BMBF (Bernstein Center for Computational Neuroscience Göttingen, T.M.) and an intramural grant from the University of Göttingen Medical School (A.C.M.).

\section{AUTHOR CONTRIBUTIONS}

The study was designed by T.M., A.C.M., A.E. and T.F. The experimental work was performed by A.C.M., T.F., D.K., D.R., G.H., N.M.C., Y.M.Y. and B.H. S.W.H. co-developed the super-resolution microscopes.

Published online at http://www.nature.com/natureneuroscience/ Reprints and permissions information is available online at http://npg.nature.com/ reprintsandpermissions/

1. Fuchs, P.A. Time and intensity coding at the hair cell's ribbon synapse. J. Physiol. 566, 7-12 (2005).
2. Moser, T., Neef, A. \& Khimich, D. Mechanisms underlying the temporal precision of sound coding at the inner hair cell ribbon synapse. J. Physiol. (Lond.) 576, 55-62 (2006).

3. Nouvian, R., Beutner, D., Parsons, T.D. \& Moser, T. Structure and function of the hair cell ribbon synapse. J. Membr. Biol. 209, 153-165 (2006).

4. Sterling, P. \& Matthews, G. Structure and function of ribbon synapses. Trends Neurosci. 28, 20-29 (2005).

5. Moser, T. \& Beutner, D. Kinetics of exocytosis and endocytosis at the cochlear inner hair cell afferent synapse of the mouse. Proc. Natl. Acad. Sci. USA 97, 883-888 (2000).

6. Khimich, D. et al. Hair cell synaptic ribbons are essential for synchronous auditory signalling. Nature 434, 889-894 (2005).

7. Griesinger, C.B., Richards, C.D. \& Ashmore, J.F. Fast vesicle replenishment allows indefatigable signalling at the first auditory synapse. Nature 435, 212-215 (2005).

8. Goutman, J.D. \& Glowatzki, E. Time course and calcium dependence of transmitter release at a single ribbon synapse. Proc. Natl. Acad. Sci. USA 104, 16341-16346 (2007).

9. Platzer, J. et al. Congenital deafness and sinoatrial node dysfunction in mice lacking class D L-type Ca2+ channels. Cell 102, 89-97 (2000).

10. Brandt, A., Striessnig, J. \& Moser, T. CaV1.3 channels are essential for development and presynaptic activity of cochlear inner hair cells. J. Neurosci. 23, 10832-10840 (2003).

11. Brandt, A., Khimich, D. \& Moser, T. Few CaV1.3 channels regulate the exocytosis of a synaptic vesicle at the hair cell ribbon synapse. J. Neurosci. 25, 11577-11585 (2005).

12. Matsubara, A., Laake, J.H., Davanger, S., Usami, S. \& Ottersen, O.P. Organization of AMPA receptor subunits at a glutamate synapse: a quantitative immunogold analysis of hair cell synapses in the rat organ of Corti. J. Neurosci. 16, 4457-4467 (1996).

13. Glowatzki, E. \& Fuchs, P.A. Transmitter release at the hair cell ribbon synapse. Nat. Neurosci. 5, 147-154 (2002).

14. Liberman, M.C. Morphological differences among radial afferent fibers in the cat cochlea: an electron-microscopic study of serial sections. Hear. Res. 3, 45-63 (1980).

15. Rutherford, M.A. \& Roberts, W.M. Frequency selectivity of synaptic exocytosis in frog saccular hair cells. Proc. Natl. Acad. Sci. USA (2006).

16. Johnson, S.L., Forge, A., Knipper, M., Munkner, S. \& Marcotti, W. Tonotopic variation in the calcium dependence of neurotransmitter release and vesicle pool replenishment at mammalian auditory ribbon synapses. J. Neurosci. 28, 7670-7678 (2008).

17. Liberman, M.C. The cochlear frequency map for the cat: labeling auditory-nerve fibers of known characteristic frequency. J. Acoust. Soc. Am. 72, 1441-1449 (1982).

18. Muller, M., von Hunerbein, K., Hoidis, S. \& Smolders, J.W. A physiological placefrequency map of the cochlea in the CBA/J mouse. Hear. Res. 202, 63-73 (2005).

19. Liberman, M.C., Dodds, L.W. \& Pierce, S. Afferent and efferent innervation of the cat cochlea: quantitative analysis with light and electron microscopy. J. Comp. Neurol. 301, 443-460 (1990); erratum 304, 341 (1991).

20. Slepecky, N.B., Galsky, M.D., Swartzentruber-Martin, H. \& Savage, J. Study of afferent nerve terminals and fibers in the gerbil cochlea: distribution by size. Hear. Res. 144 124-134 (2000).

21. Francis, H.W., Rivas, A., Lehar, M. \& Ryugo, D.K. Two types of afferent terminals innervate cochlear inner hair cells in C57BL/6J mice. Brain Res. 1016, 182-194 (2004).

22. Liberman, M.C. Single-neuron labeling in the cat auditory nerve. Science 216, 1239-1241 (1982).

23. Taberner, A.M. \& Liberman, M.C. Response properties of single auditory nerve fibers in the mouse. J. Neurophysiol. 93, 557-569 (2005)

24. Merchan-Perez, A. \& Liberman, M.C. Ultrastructural differences among afferent synapses on cochlear hair cells: correlations with spontaneous discharge rate. J. Comp. Neurol. 371, 208-221 (1996).

25. Ruel, J. et al. Dopamine inhibition of auditory nerve activity in the adult mammalian cochlea. Eur. J. Neurosci. 14, 977-986 (2001).

26. Hell, S. \& Stelzer, E.H.K. Properties of a 4Pi-confocal fluorescence microscope. J. Opt. Soc. Am. A 9, 2159-2166 (1992)

27. Egner, A., Jakobs, S. \& Hell, S.W. Fast 100-nm resolution three-dimensional microscope reveals structural plasticity of mitochondria in live yeast. Proc. Natl. Acad. Sci. USA 99, 3370-3375 (2002).

28. Hell, S.W. \& Wichmann, J. Breaking the diffraction resolution limit by stimulated emission: stimulated emission depletion microscopy. Opt. Lett. 19, 780-782 (1994).

29. Muller, M. The cochlear place-frequency map of the adult and developing Mongolian gerbil. Hear. Res. 94, 148-156 (1996).

30. Wu, Y.C., Tucker, T. \& Fettiplace, R. A theoretical study of calcium microdomains in turtle hair cells. Biophys. J. 71, 2256-2275 (1996).

31. Martinez-Dunst, C., Michaels, R.L. \& Fuchs, P.A. Release sites and calcium channels in hair cells of the chick's cochlea. J. Neurosci. 17, 9133-9144 (1997).

32. Masugi-Tokita, M. et al. Number and density of AMPA receptors in individual synapses in the rat cerebellum as revealed by SDS-digested freeze-fracture replica labeling. J. Neurosci. 27, 2135-2144 (2007).

33. Tokuoka, H. \& Goda, Y. Activity-dependent coordination of presynaptic release probability and postsynaptic GluR2 abundance at single synapses. Proc. Natl. Acad. Sci. USA 105, 14656-14661 (2008).

34. Rostaing, P., Weimer, R.M., Jorgensen, E.M., Triller, A. \& Bessereau, J.L. Preservation of immunoreactivity and fine structure of adult $C$. elegans tissues using high-pressure freezing. J. Histochem. Cytochem. 52, 1-12 (2004).

35. Roberts, W.M., Jacobs, R.A. \& Hudspeth, A.J. Colocalization of ion channels involved in frequency selectivity and synaptic transmission at presynaptic active zones of hair cells. J. Neurosci. 10, 3664-3684 (1990).

36. Tucker, T. \& Fettiplace, R. Confocal imaging of calcium microdomains and calcium extrusion in turtle hair cells. Neuron 15, 1323-1335 (1995). 
37. Schnee, M.E., Lawton, D.M., Furness, D.N., Benke, T.A. \& Ricci, A.J. Auditory hair cellafferent fiber synapses are specialized to operate at their best frequencies. Neuron 47, 243-254 (2005)

38. Beutner, D., Voets, T., Neher, E. \& Moser, T. Calcium dependence of exocytosis and endocytosis at the cochlear inner hair cell afferent synapse. Neuron 29, 681-690 (2001).

39. Rodriguez-Contreras, A. \& Yamoah, E.N. Direct measurement of single-channel $\mathrm{Ca}^{2+}$ currents in bullfrog hair cells reveals two distinct channel subtypes. J. Physiol. (Lond.) 534, 669-689 (2001).

40. Frank, T., Khimich, D., Neef, A. \& Moser, T. Mechanisms contributing to synaptic $\mathrm{Ca}^{2+}$ signals and their heterogeneity in hair cells. Proc. Natl. Acad. Sci. USA (in the press).

41. Issa, N.P. \& Hudspeth, A.J. Clustering of $\mathrm{Ca}^{2+}$ channels and $\mathrm{Ca}^{2+}$-activated $\mathrm{K}^{+}$channels at fluorescently labeled presynaptic active zones of hair cells. Proc. Natl. Acad. Sci. USA 91, 7578-7582 (1994).

42. Zenisek, D., Davila, V., Wan, L. \& Almers, W. Imaging calcium entry sites and ribbon structures in two presynaptic cells. J. Neurosci. 23, 2538-2548 (2003).
43. Tsuji, J. \& Liberman, M.C. Intracellular labeling of auditory nerve fibers in guinea pig: central and peripheral projections. J. Comp. Neurol. 381, 188-202 (1997).

44. Ehret, G. Age-dependent hearing loss in normal hearing mice. Naturwissenschaften $\mathbf{6 1}$, 506-507 (1974).

45. Liberman, M.C. et al. Prestin is required for electromotility of the outer hair cell and for the cochlear amplifier. Nature 419, 300-304 (2002).

46. Ehret, G. Common rules of communication sound perception. in Behavior and Neurodynamics for Auditory Communication (ed. J.S. Kanwal \& G. Ehret) 85-114 (Cambridge University Press, Cambridge, 2006).

47. Fuchs, P.A., Glowatzki, E. \& Moser, T. The afferent synapse of cochlear hair cells. Curr. Opin. Neurobiol. 13, 452-458 (2003).

48. Nusser, Z. AMPA and NMDA receptors: similarities and differences in their synaptic distribution. Curr. Opin. Neurobiol. 10, 337-341 (2000).

49. Johnson, S.L. \& Marcotti, W. Biophysical properties of CaV1.3 calcium channels in gerbil inner hair cells. J. Physiol. (Lond.) 586, 1029-1042 (2008).

50. Harke, B. et al. Resolution scaling in STED microscopy. Opt. Express 16, 4154-4162 (2008). 


\section{Supplementary Information for:}

\section{Tuning of Synapse Number, Structure and Function in}

\section{the Cochlea}

Alexander C. Meyer, Thomas Frank, Darina Khimich, Gerhard Hoch, Dietmar Riedel,

Nikolai M. Chapochnikov, Yury M. Yarin, Benjamin Harke, Stefan Hell, Alexander Egner

and Tobias Moser

Running title: Synaptic tuning in the cochlea

\section{Contains:}

Supplementary Data 1 Further analysis of hair cell synapse distribution

(including Supplementary Fig. 1, Supplementary Table 1, Supplementary Fig. 2)

Supplementary Data 2 Comparison of hair cell synapse ultrastructure following high pressure rapid freezing and chemical fixation

(including Supplementary Fig. 3, Supplementary Methods)

Supplementary Data 3 Further Analysis of 4Pi and STED data on synapse structure (including Supplementary Fig. 4, Supplementary Fig. 5)

Supplementary Data 4 Further analysis of $\mathrm{I}_{\mathrm{Ca}}$ properties of IHCs as a function of tonotopic position (including Supplementary Fig. 6, Supplementary Table 2)

Supplementary Table 3 Analysis of the variance among synaptic $\mathrm{Ca}^{2+}$ microdomains Supplementary Movie 1 accompanying information for 3D STED imaging of postsynaptic AMPA receptor clusters 


\section{Supplementary Data 1 Further analysis of hair cell synapse distribution}
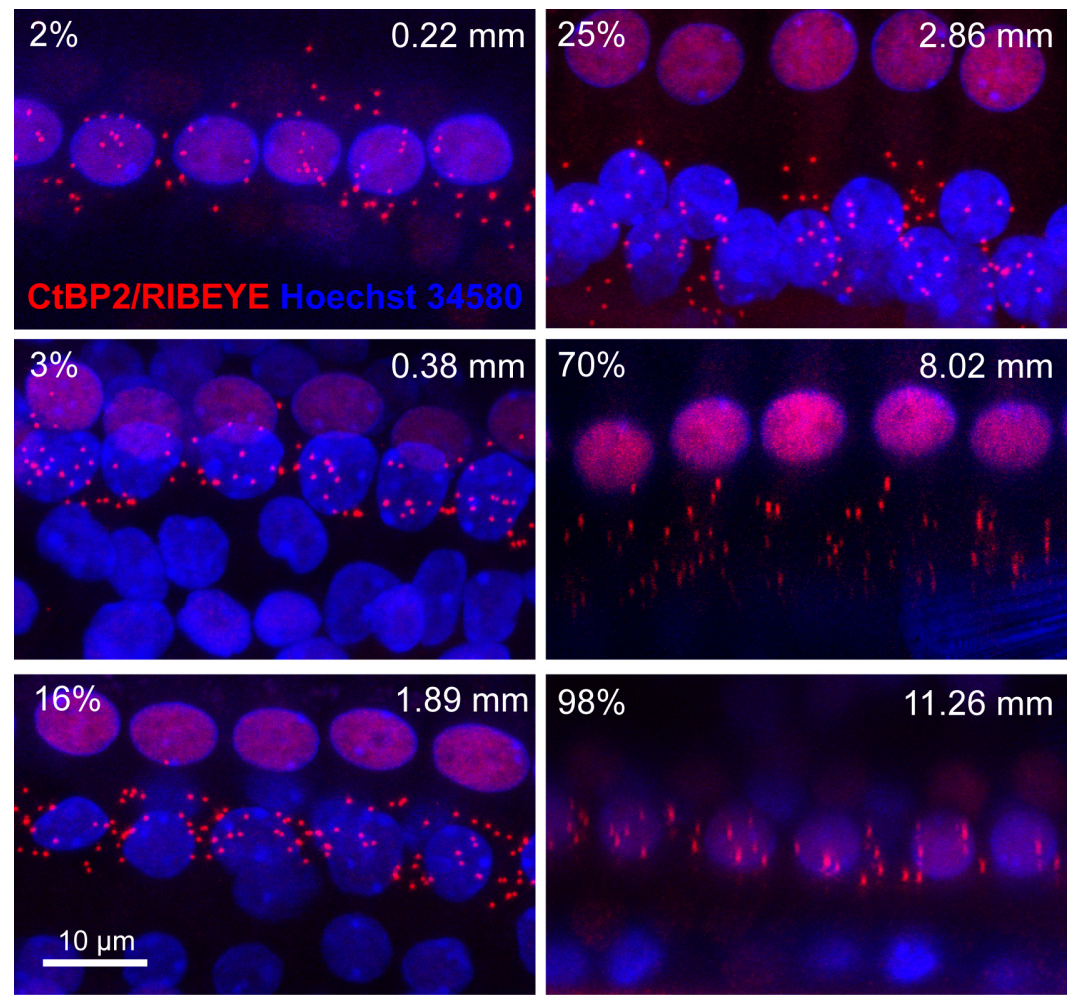

Supplementary Figure 1 Ribbon synapse number in a low-frequency hearing animal (gerbil)

Projections of confocal stacks of immunolabeled inner hair cell afferent synapses at different tonotopic locations in the gerbil. (red: anti-CtBP2/RIBEYE; blue: nuclear stain by Hoechst 34580). Percentage indicates the location of the respective image along the tonotpic axis ( $0 \%$ corresponding to the apical end, and $100 \%$ to the basal end of the cochlea). Presynaptic ribbons were counted in each stack and related to the number of IHCs.

Supplementary Table 1 Fit coefficients describing the relation between ribbon-number (y) and tonotopic position (x) (2nd order polynomial fit function: $y=c_{0}+c_{1} x+c_{2} x^{2}$, with $\mathrm{x}$ being the tonotopic location in $\%$ of total cochlear length).

\begin{tabular}{|l|l|l|}
\hline Fit coefficient & Mouse (coefficient \pm SD) & Gerbil (coefficient \pm SD) \\
\hline$c_{0}$ & $8.0 \pm 0.3$ & $15.1 \pm 1.5$ \\
\hline$c_{1}$ & $0.38 \pm 0.02$ & $0.37 \pm 0.08$ \\
\hline$c_{2}$ & $-0.0040 \pm 0.0002$ & $-0.0047 \pm 0.0008$ \\
\hline
\end{tabular}



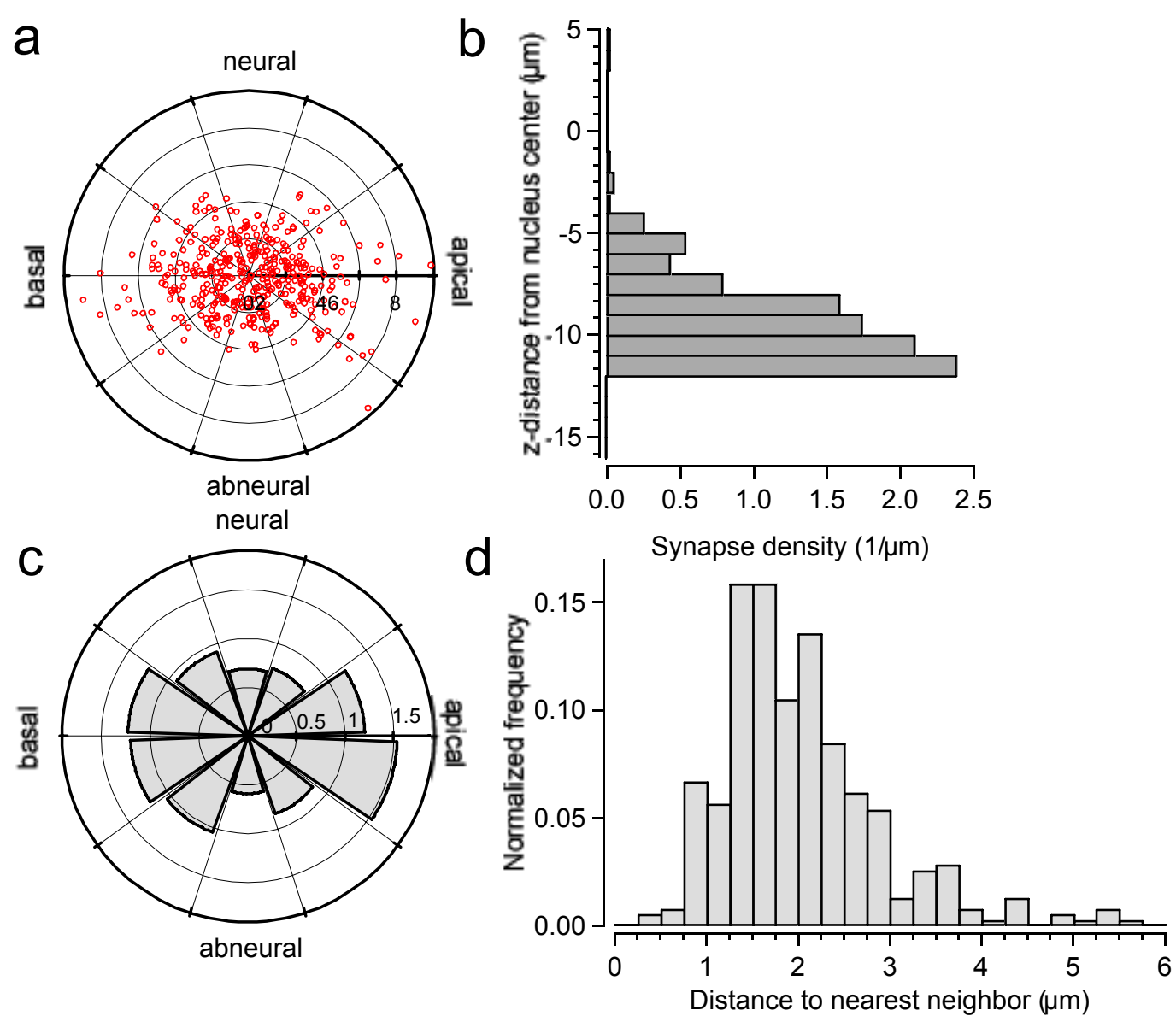

\section{Supplementary Figure 2 Spatial distribution of afferent synapses within IHCs at the apex}

Identical analysis of data as shown in Figure 2 of the main manuscript, but for synapses from IHCs at the apex of the cochlea. Synapses show a slight tendency to accumulate along the apical-basal axis (a and c). The absolute number of synapses per sector is decreased as the number per cell is lower in this region (c). IHCs tend to be shorter at the apex compared to mid-cochlear, resulting in an accumulation of synapses at $12 \mu \mathrm{m}$ (average $9.2 \pm 2.4 \mu \mathrm{m}$ ) rather than $15 \mu \mathrm{m}$ (average $11.6 \pm 3.1 \mu \mathrm{m}$, mid-cochlear) from the nucleus (b). Inter-synaptic distance, however, is comparable (d) (mean $=2.0 \pm 0.9 \mu \mathrm{m}$ and $2.0 \pm 1.0 \mu \mathrm{m}$, apex and mid-cochlear, respectively). 


\section{Supplementary Data 2 \\ Comparison of hair cell synapse ultrastructure following high pressure rapid freezing and chemical fixation}
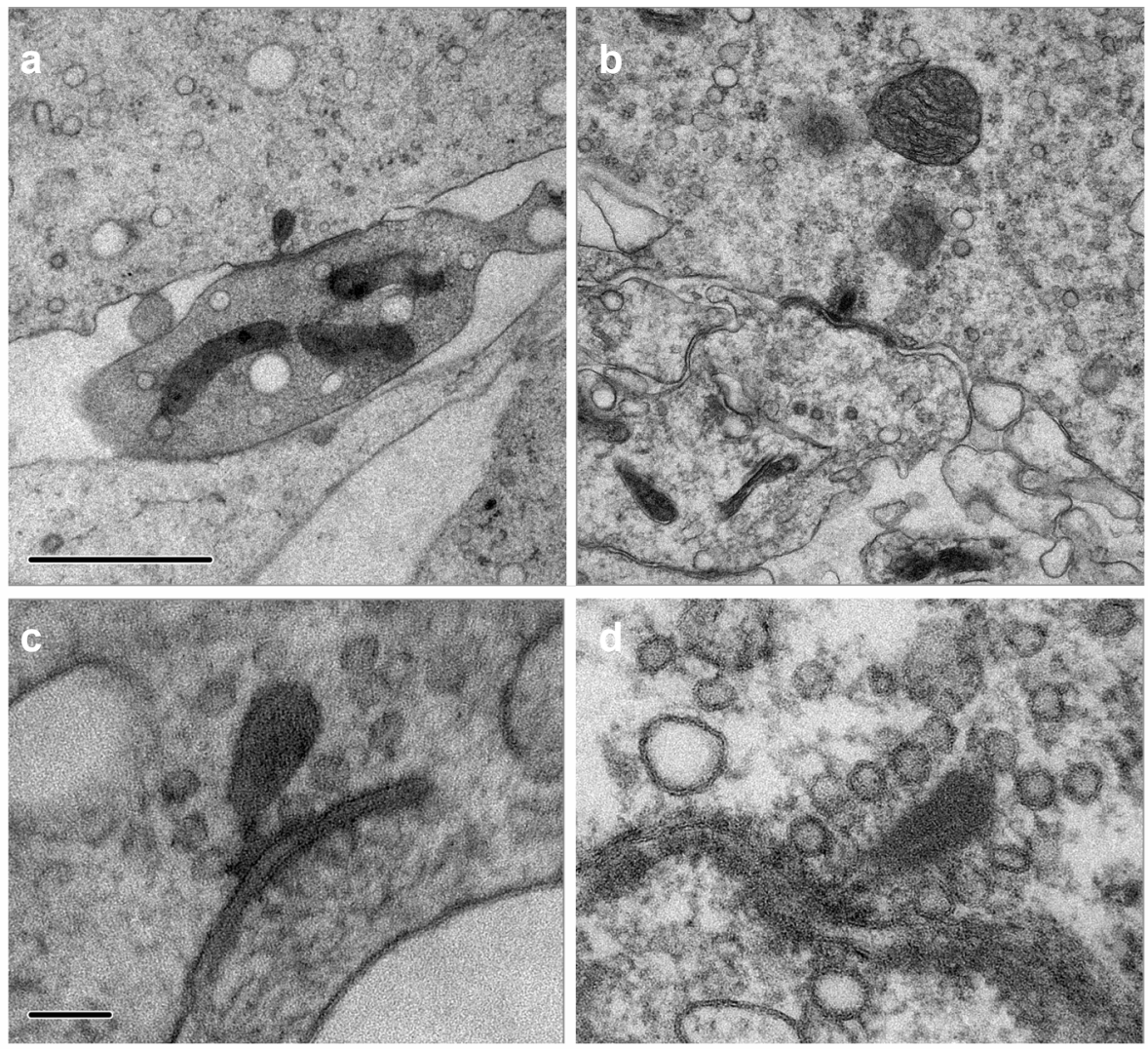

Supplementary Figure 3 Comparison of IHC ribbon synapse ultrastructure between high pressure rapid freezing and aldehyde fixation.

a and c, High pressure frozen and freeze substituted organs of Corti. b and d chemically fixed organs of Corti embedded in Agar 100 at room temperature. Scale bars: a and b: $1 \mu \mathrm{m}, \mathrm{c}$ and $\mathrm{d}: 100 \mathrm{~nm}$.

To address the question whether the structure of IHC ribbon synapses in electron microscopy (EM) is altered by chemical fixation and the embedding method used, we compared two different protocols. The organs of Corti were (1) embedded in Agar 100 following conventional chemical fixation with glutaraldehyde at room temperature, and (2) physical fixation with high pressure rapid freezing followed by freeze substitution (see Supplementary Methods section below). The gross structure of the ribbon synapses was not different between the two methods. 
IHCs showed well preserved cytoplasmic structures after high pressure fixation of the organ of Corti. Cytoplasm did not appear to be washed out and was evenly distributed throughout the cell. Membranes of organelles as well as the plasma-membrane were smooth and clearly visible. However, the contrasts of the membranes after substitution using tannic acid and $\mathrm{OsO}_{4}$ remained weak.

IHCs in chemically fixed organs of Corti showed some wash-out of cytoplasm and crinkled plasma-membranes with clearly visible bilayer structure. The synaptic vesicle diameter estimates in chemically fixed synapses (41.4 nm [apex] and $44.6 \mathrm{~nm}$ [mid-cochlea], Table 1 of main manuscript) were in line with our previous estimate ${ }^{2}$ (mean outer diameter: $43.1 \mathrm{~nm}$ ) but smaller than in high pressure frozen samples (48.7 $\mathrm{nm}$, see Table 1 of main manuscript). Possible reasons for this difference include: (1) shrinkage due to chemical fixation and (2) the use of tannic acid for staining of the "frozen" vesicles (tannic acid precipitates in the outer leaflet of the membrane). For evaluation of synaptic morphology at different tonotopic positions, it is essential to obtain a homogeneous embedding quality over the entire apical coil of the organ of Corti. This was not regularly achieved in high pressure frozen and freeze substituted samples (cryoartifacts). Therefore, the evaluation was performed using the conventional room temperature embedding method, resulting in a comparable preservation of the structures over the entire organ.

\section{Supplementary Methods: Electron microscopy}

Organs of Corti from p17-21 old C57B1/6 mice were fixed immediately after dissection with $2.5 \%$ glutaraldehyde in HEPES-buffered Hanks solution for $30 \mathrm{~min}$ at room temperature. Thereafter, the samples were fixed over night at $4{ }^{\circ} \mathrm{C}$ in $2 \%$ glutaraldehyde in $0.1 \mathrm{M}$ cacodylic buffer at $\mathrm{pH}$ 7.4. After an additional fixation in $0.1 \% \mathrm{OsO}_{4}$, the samples were stained with $1 \%$ uranyl acetate, dehydrated in a series of ethanol and finally in propylene oxide. They were then embedded in Agar 100 (Science Services, Munich, Germany). Consecutive ultrathin sections $(80 \mathrm{~nm})$ were mounted on slot grids counterstained with $1 \%$ uranyl acetate and lead citrate and examined using a Philips CM 120 BioTwin transmission electron microscope (Philips Inc., Eindhoven, Netherlands). Images were taken with a TemCam F224A camera (TVIPS, Gauting, Germany) at 
20,000-fold magnification. Alternatively, organs of Corti were high pressure frozen in a Baltec HPM010 and embedded using a Leica EMAFS. The samples were freeze substituted in Agar 100 using acetone containig 0.1\% tannic acid and $\mathrm{OsO}_{4}$ (Electron Microscopy Science). Sections were considered for analysis only, if the postsynaptic density was sharply delimited.

\section{Supplementary Methods: Auditory brainstem responses}

Experiments were performed as described in ref. ${ }^{1}$, complied with national animal care guidelines, and were approved by the University of Goettingen Board for animal welfare and the animal welfare office of the state of Lower Saxony. In brief, animals were anaesthetized intraperitoneally with a combination of ketamine $\left(125 \mathrm{mg} \mathrm{kg}^{-1}\right)$ and xylazin $\left(2.5 \mathrm{mg} \mathrm{kg}^{-1}\right)$ and the core body temperature was maintained constant at $37^{\circ} \mathrm{C}$. For stimulus generation, presentation and data acquisition we used the TDT III Systems (Tucker-Davis-Technologies, Ft Lauderdale, FL) run by BioSig32 software (TDT). Tone bursts $\left(4 / 8 / 12 / 16 / 24 / 32 / 48 / 64 \mathrm{kHz}, 10 \mathrm{~ms}\right.$ plateau, $1 \mathrm{~ms} \cos ^{2}$ rise/fall, calibrated and provided in $\mathrm{dB}$ SPL rms) were applied at $20 \mathrm{~Hz}$ in the free field ipsilaterally using a JBL 2402 speaker (JBL GmbH \& Co., Neuhofen, Germany). The difference potential between vertex and mastoid intradermal needles was amplified (5 $10^{4}$-times), filtered (low pass: 4 $\mathrm{kHz}$, high pass: $100 \mathrm{~Hz}$ ) and sampled at a rate of $50 \mathrm{kHz}$ for $20 \mathrm{~ms}$. Trials were repeated 2x2000 times to obtain two mean ABR traces for each sound intensity. Hearing threshold was determined with $10 \mathrm{~dB}$ precision as the lowest stimulus intensity that evoked a reproducible response waveform in both traces, as judged by visual inspection. 


\section{Supplementary Data 3 Further Analysis of 4Pi and STED data on synapse structure}

This document supplements the quantitative light microscopy analysis of the inner hair cell synapse presented in Figure 3 of the main manuscript. Supplementary Figure 4 illustrates further comparative analysis of ribbon size using 4Pi and STED microscopy. Supplementary Figure 5 provides further analysis and modeling of the images of immunolabeled presynaptic $\mathrm{Ca}_{\mathrm{V}} 1.3 \mathrm{Ca}^{2+}$ channel clusters obtained by STED microscopy.
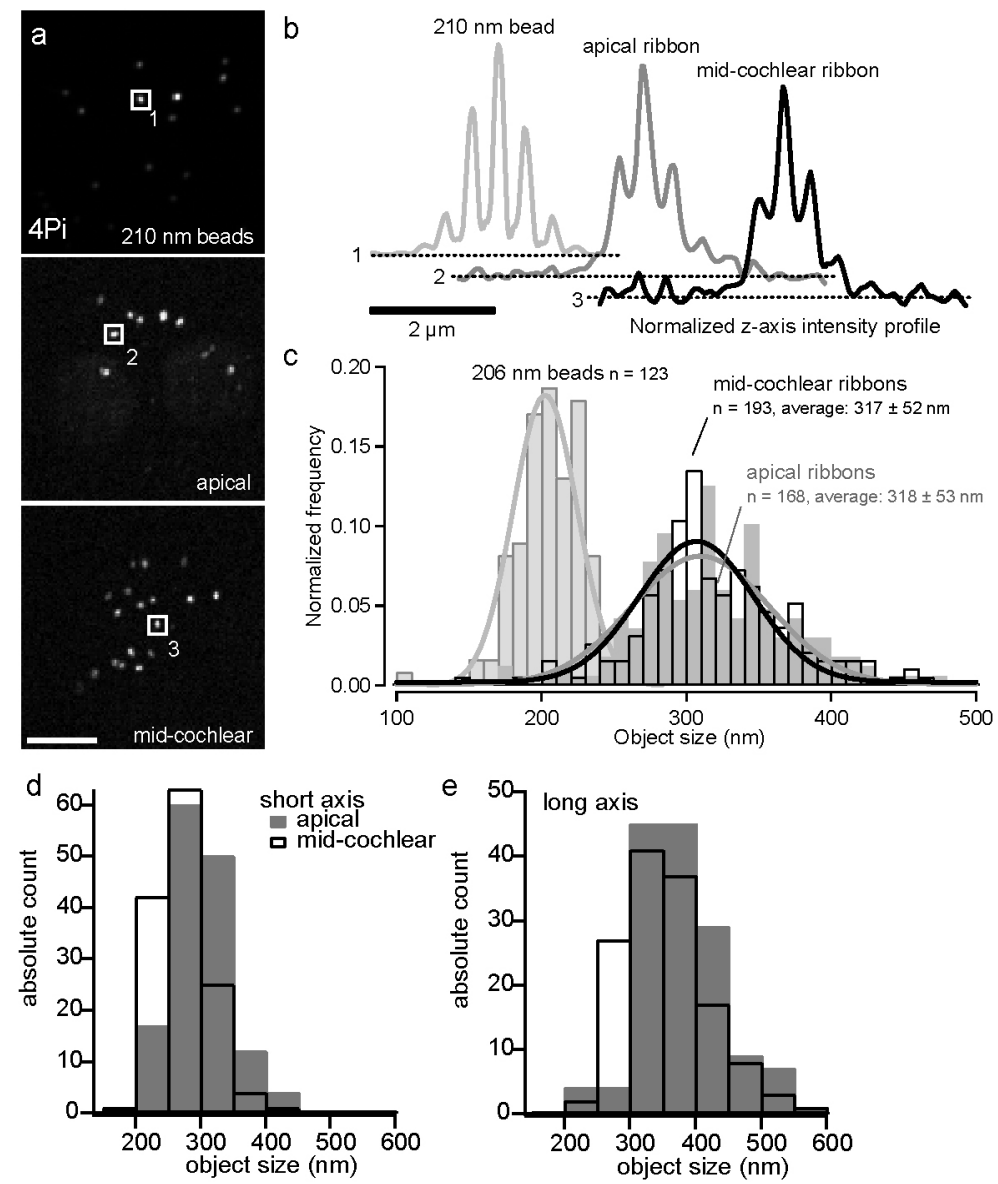

$4 \mathrm{Pi}$

STED

Supplementary Figure 4 Comparison of apparent ribbon size by 4Pi and STED microscopy of immunolabeled ribbons between synapses of apical and mid-cochlear IHCs.

a, 4Pi images of fluorescent beads (206 nm, top), CtBP2/RIBEYE immunolabeled ribbons of apical (middle) and mid-cochlear IHCs of NMRI mice (bottom, scale bar: 10 $\mu \mathrm{m})$. The CtBP2/RIBEYE labeled IHC nuclei are faintly visible. b, axial intensity profiles of the 3 fluorescent objects boxed in (a), obtained from stacks of 4Pi image 
sections as used for estimation of axial thickness ${ }^{3}$. Note that the minima, separating the maxima and side lobes of the ribbons are shallower than those for the bead, indicating a larger size of the ribbons. c, axial thickness distributions for beads and ribbons of both locations. The size estimates exceed our previously published estimate of apparent ribbon size in IHCs from the apical coil of 8-week-old mice ${ }^{4}(260 \mathrm{~nm})$, which turned out to be too small because the size of the fluorescent beads used for calibration was smaller than specified by the provider. The estimates were brought to agreement, when the previous data were re-calibrated with an EM-controlled bead size (308 \pm 88 [SD] nm, n=239).

d, e, STED: histograms of short (d) and long (e) axes approximated as the full widths at half maximum (FWHM) of two orthogonal Gaussian functions to the STED images of immunofluorescent ribbons from apical and mid-cochlear IHCs. As described in the main manuscript, sections were taken at the maximum intensity z-level, afore determined by confocal microscopy.

\section{Comparison of long and short axes of Cav1.3-immunofluorescent spots imaged by STED microscopy: further analysis and modeling}

Asking which geometric shape of the immunolabeled $\mathrm{Ca}^{2+}$ channel clusters underlies the distributions of short and long axes obtained from the analysis of STED microscopy images, we simulated projections of ellipsoids, assuming a random orientation of the clusters (synapses are distributed in a nearly hemispherical cap formed by the basolateral pole of the IHC, see Fig. 2 of the main manuscript). The ellipsoidal model was chosen for practical reasons, and considering the thickness of the plasma membrane as well as the size of antibodies, we fixed the ellipsoids' shortest axis (termed: "thickness") to $30 \mathrm{~nm}$. To generate random orientations, an ellipsoid with its principal axes parallel to the coordinates $\mathrm{x}, \mathrm{y}, \mathrm{z}$ was stochastically rotated around the $\mathrm{z}$ axis and then the $\mathrm{z}$ axis of the ellipsoid was rotated toward a unit vector randomly picked on a unit sphere ${ }^{3}$.

To model the experimental data, we convolved the randomly oriented ellipsoids with a Point Spread Function (PSF) model and determined the minimum and the maximum FWHM of the resulting image numerically. As measuring the PSF at the position of the synapses (nearly halfway inside the tissue sample) was not feasible, we 
approximated it by a 3D Gaussian with a FWHM of $150 \pm 20 \mathrm{~nm}$ (mean $\pm \mathrm{sd}$ ) in the $\mathrm{x}$ and $\mathrm{y}$ directions and $500 \mathrm{~nm}$ in the $\mathrm{z}$ direction. The $\mathrm{FWHM}_{\mathrm{x}, \mathrm{y}}$ values were chosen as the arithmetic mean of measurements of $100 \mathrm{~nm}$ beads, imaged in front (x: $121 \pm 16$ (mean \pm SD), y: $126 \pm 16 \mathrm{~nm}$ ) and behind (x: $173 \pm 24, \mathrm{y}: 164 \pm 28 \mathrm{~nm}$ ) the sample.

The ellipsoid was varied in size, which together with different orientations and variance in the PSF causes the variability of the observed data. In order to find the ellipsoid that would best fit the experiments, we also implemented a certain variance of the ellipsoids' axes (both Gaussian and Gamma distributed). Criteria for identifying the best fitting ellipsoid included comparison of the mean and the variances of the determined long and short axes, as well as the correlation between these two axes. In Supplementary Figure 5, two simulations, each performed with a different set of parameters, are presented. In both cases, the two longest axes were assumed to be Gamma distributed. Note, as the data does not provide information about the thickness of the cluster beyond saying that it smaller than the microscope's resolution limit, there was no need for varying the size of the shortest axis. Systematically, the high correlation of the long and short axis estimates in the experimental data could not be matched in the simulation, even when perfectly correlating the sizes of the two longest axes of the ellipsoid.

From the simulations we conclude:

- Considering ellipsoids with different lateral extent but fixed ratio of the two long axes (oblate: 1 or scalene $\neq 1$ ) the best fit of the experimental data was obtained with an oblate-shaped ellipsoid ( $\approx 420 \pm 126 \mathrm{~nm}, \approx 420 \pm 126 \mathrm{~nm}, 30 \mathrm{~nm}): 1)$ scalene shaped ellipsoids did not generate enough points close to the unity line and 2) oblate shaped ellipsoids fit better the observed means and variances of the axes. While this may describe the average cluster's shape we anticipate a range of long axes ratios for $\mathrm{Ca}^{2+}$ channel clusters.

- The skewness in the experimental axis estimates (data not shown) could not be reproduced by assuming Gaussian distributed axes of the ellipsoids. A positively skewed distribution, such as the Gamma distribution was more appropriate to match the data (Supplementary Figure $5 \mathrm{~b}$ and $\mathrm{c}$ ). 
- Comparing experiments with the simulations one notices that points with large long axes and small short axes are missing in the experimental distribution. One possible explanation for this is that analysis of the images by 2D Gaussian fitting may overestimate the short axis for long curved clusters. This would also explain why the correlation of the data is not sufficiently matched by our simulations and why the model systematically generated smaller short axis estimates than found in the data.

a. Experimental Data
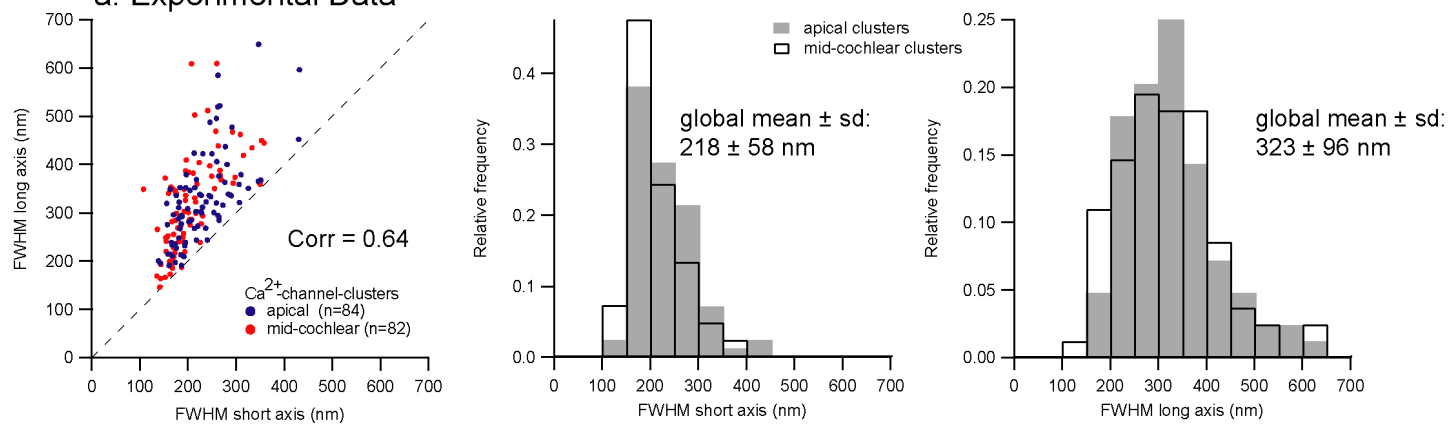

b. Model 1 : oblate : $420 \pm 126 \mathrm{~nm}$
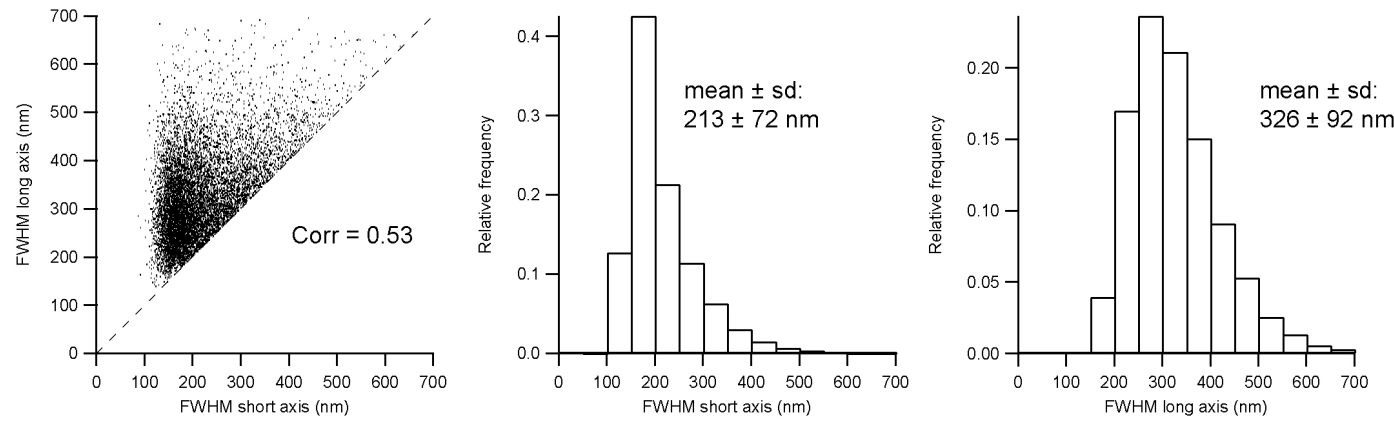

c. Model 2 : scalene : $490 \pm 147 \times 320 \pm 112 \mathrm{~nm}$ (with constant ratio between the 2 long axis)
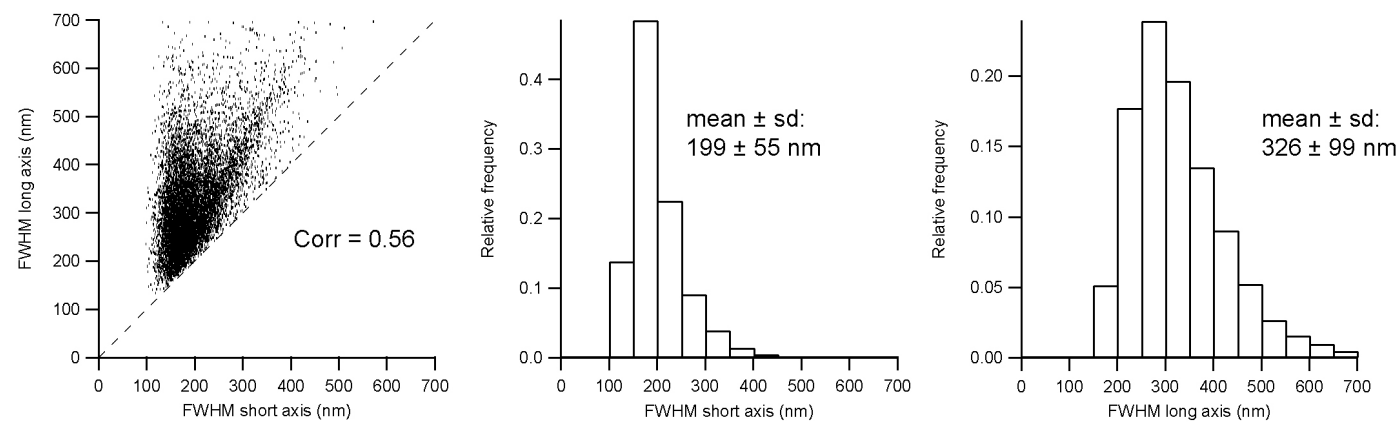

\section{Supplementary Figure 5}

(a) provides the experimental Full Widths at Half Maximum (FWHM) estimates, obtained by fitting a 2D Gaussian to the STED images of immunofluorescent presynaptic 
$\mathrm{Ca}_{\mathrm{v}} 1.3 \mathrm{Ca}^{2+}$ channel clusters of apical and mid-cochlear IHCs (scatter plot as in Fig. $3 \mathrm{~g}$ of the main manuscript (left, for comparison to model distributions), histograms of short (middle) and long (right) axes). As described in the main manuscript, sections were taken at the maximum intensity z-level.

(b) simulation assuming an ellipsoid with two identical long axes (oblate: $420 \pm 126 \mathrm{~nm}$, $420 \pm 126 \mathrm{~nm}, 30 \mathrm{~nm})$

(c) simulation assuming an ellipsoid with three different axes (scalene: $490 \pm 147 \mathrm{~nm}$, $320 \pm 112 \mathrm{~nm}, 30 \mathrm{~nm})$. 


\section{Supplementary Data 4 Further analysis of $I_{C a}$ properties of IHCs as a function of}

\section{tonotopic position}

Data were obtained in standard whole-cell recordings under augmenting conditions (10 $\mathrm{mM}\left[\mathrm{Ca}^{2+}\right]_{\mathrm{e}}, 5 \mu \mathrm{M}$ BayK; see also methods section of the main manuscript).

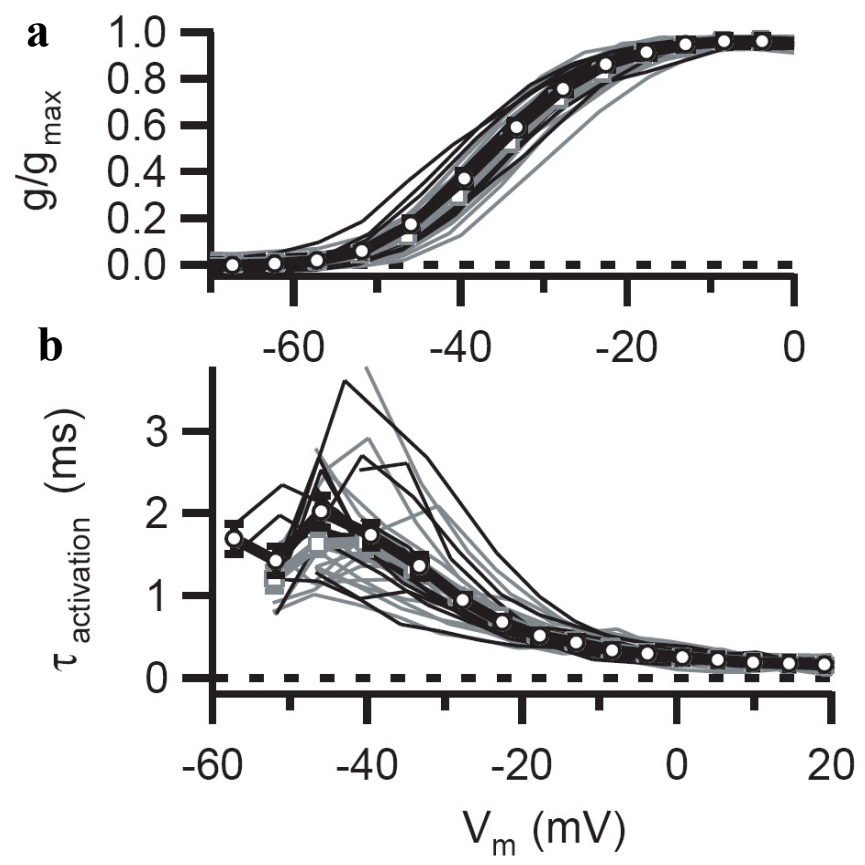

Supplementary Figure 6 Comparison of voltage dependence and kinetics of channel activation between apical and mid-cochlear IHCs.

a, voltage dependence of (fractional) calcium conductance activation: Figure shows average (thick) and individual (thin) traces for apical (gray, $\mathrm{n}=19$ ) and mid-cochlear (black, $n=14$ ) IHCs. Steady-state current was estimated as the average current over the last $7 \mathrm{~ms}$ of a $10 \mathrm{~ms}$ voltage step to the potential indicated. Conductance-voltage relationships were derived from steady-state I-V curves (see Fig. $4 \mathrm{~d}$ of the main manuscript) by dividing current by the driving force $\left(\mathrm{V}-\mathrm{V}_{\text {rev }}\right)$. The reversal potential $\left(\mathrm{V}_{\text {rev }}\right)$ of the calcium current was determined empirically by fitting a line to the I-V in the range between $-5 \mathrm{mV}$ and $+25 \mathrm{mV}$ and extrapolating to the zero-crossing of the fit result. b, time constants of activation for $\mathrm{I}_{\mathrm{Ca}}$ at different membrane potentials. Thick lines represent average, thin lines individual traces (gray: apical, black: mid-cochlear; same cells as in Fig. 4d of main manuscript). Activation of current traces was fit with a single exponential function with an exponent of activation of 2 . 
Fluctuation analysis was performed as described in the methods section of the main manuscript. Curve fitting used weighting according to the error-covariance of the variance (eGLS ${ }^{1}$ ) with binning the variance in $100 \mu$ s bins.

Supplementary Table 2 Summarizing comparison of whole-cell $\mathrm{I}_{\mathrm{Ca}}$ properties between apical and mid-cochlear IHCs (augmenting conditions: $10 \mathrm{mM}\left[\mathrm{Ca}^{2+}\right]_{e}, 5 \mu \mathrm{M}$ BayK8644)

\begin{tabular}{|c|c|c|}
\hline & Apical & Mid-cochlear \\
\hline Peak steady-state $I_{C a}(p A)(p=0.07)$ & $471 \pm 36$ & $587 \pm 47$ \\
\hline$G_{\max }(n S)(p=0.11)$ & $9.4 \pm 0.8$ & $11.5 \pm 1.0$ \\
\hline$V_{1 / 2}(m V)(p=0.05)$ & $-35.0 \pm 0.6$ & $-36.5 \pm 0.8$ \\
\hline Slope $(m V)(p=0.37)$ & $6.0 \pm 0.1$ & $6.1 \pm 0.1$ \\
\hline $\mathbf{N}$ (cells) & 19 & 14 \\
\hline Channel number $\left(\mathrm{N}_{\mathrm{Ca}}\right)(\mathrm{p}<0.01)$ & $1366 \pm 67$ & $1828 \pm 137$ \\
\hline $\mathrm{i}_{\mathrm{Ca}}(\mathrm{pA})(\mathrm{p}=0.04)$ & $-0.67 \pm 0.02$ & $-0.60 \pm 0.02$ \\
\hline$p_{o, \max }(p<0.001)$ & $0.84 \pm 0.01$ & $0.91 \pm 0.01$ \\
\hline$I_{\max }(p=0.01)$ & $-784 \pm 57$ & $-1004 \pm 54$ \\
\hline $\mathbf{N}$ (cells) & 13 & 10 \\
\hline
\end{tabular}

Means are quoted \pm s.e.m. (standard error of the mean). If applicable (i.e., as determined by normality of the distribution (Jarque-Bera test) and "equal variances between" (F-test) the two samples), an unpaired, two-tailed t-test was used to compare the two samples. In all other cases, a Mann-Whitney-Wilcoxon test was employed as a statistical test.

\section{References}

1. Pauli-Magnus, D., et al. Detection and differentiation of sensorineural hearing loss in mice using auditory steady-state responses and transient auditory brainstem responses. Neuroscience 149, 673-684 (2007).

2. Neef, A., et al. Probing the mechanism of exocytosis at the hair cell ribbon synapse. J Neurosci 27, 12933-12944 (2007).

3. Egner, A., Jakobs, S. \& Hell, S.W. Fast 100-nm resolution three-dimensional microscope reveals structural plasticity of mitochondria in live yeast. Proc Natl Acad Sci US A 99, 3370-3375 (2002).

4. Khimich, D., et al. Hair cell synaptic ribbons are essential for synchronous auditory signalling. Nature 434, 889-894 (2005).

5. Weisstein, Eric W. "Sphere Point Picking." From MathWorld--A Wolfram Web Resource. http://mathworld.wolfram.com/SpherePointPicking.html 


\section{Supplementary Table 3 Analysis of the variance among synaptic $\mathrm{Ca}^{2+}$ microdomains}

Data of apical and mid-cochlear synapses were pooled for variance analysis as they did not differ significantly from each other (see Table 2 of main manuscript). For estimation of the peak fluorescence change we used two strategies, depending on the type of image aqcuisition: (1) for spot detection, we recorded from a series of $130 \mathrm{~nm}$ laterally offset locations and selected the trace with the maximal fluorescence increase (see Fig. 5 of main manuscript), whereas (2) for line scans, we fit Gaussian functions to the timeaveraged $\mathrm{X}$ and $\mathrm{Y}$ line scan data and estimated the maximal fluorescence increase as the peak amplitude of the obtained fit. Likewise, the FWHM was derived from this Gaussian fit. $\mathrm{V}_{1 / 2}(\Delta \mathrm{F}-\mathrm{V})$ and slope $(\Delta \mathrm{F}-\mathrm{V})$ were obtained from fitting a Boltzmann function to fluorescence-voltage relationships (see Fig. $5 \mathrm{~d}$ of main manuscript), and are compared to the estimates $\left[\mathrm{V}_{1 / 2}(\mathrm{I}-\mathrm{V})\right.$ and slope $\left.(\mathrm{I}-\mathrm{V})\right]$ obtained the same way for the whole-cell $\mathrm{Ca}^{2+}$ currents of IHCs from the same tonotopic region. CV: coefficient of variation.

\begin{tabular}{|c|c|}
\hline & Dispersion \\
\hline Peak $\Delta F$ (spot detection) & CV: $0.63(n=65)$ \\
\hline (X) Peak $\Delta F$ (line-scan) & CV: $0.61(n=45)$ \\
\hline (Y) Peak $\Delta F$ (line-scan) & CV: $0.68(n=42)$ \\
\hline (X) FWHM (line-scan) & CV: $0.32(n=45)$ \\
\hline (Y) FWHM (line-scan) & CV: $0.39(n=42)$ \\
\hline$V_{1 / 2}(\Delta F-V)$ & SD: $5.8 \mathrm{mV}(\mathrm{n}=37)$ \\
\hline Slope $(\Delta F-V)$ & CV: $0.27(n=37)$ \\
\hline V1/2 (whole-cell I-V), purely intercellular & SD: $2.9 \mathrm{mV}^{1}(\mathrm{~N}=33)$ \\
\hline Slope (whole-cell I-V), purely intercellular & CV: $0.08^{1}(\mathrm{~N}=33)$ \\
\hline
\end{tabular}

\footnotetext{
${ }^{1}$ Value for both apical $(n=19)$ and basal $(n=14)$ IHCs
} 


\section{Supplementary Movie 1}

\section{D STED imaging of postsynaptic AMPA receptor clusters}

Left, Voltex-rendering of a 3D STED image of a postsynaptic AMPA receptor cluster (immunolabeled for GluR2/3, resolution $\sim 150 \mathrm{~nm}$, raw data). The gradient of the fluorescence intensity as well as the off-center maximum are clearly visible. Right, in order to emphasize the "ring-like" appearance and the curved shape the voltex-rendering is combined with an isosurface view. The isosurface has been generated by using a seed fill algorithm (magic wand, Amira, Visage Imaging) utilizing 64\% and 100\% of the maximum intensity as the lower and upper threshold respectively. 


\section{General Discussion}

\subsection{Comparison to $\mathrm{Ca}^{2+}$ Microdomains in Other Hair Cell Types}

\subsubsection{General Properties}

The presynaptic $\mathrm{Ca}^{2+}$ microdomains that we observed in IHCs of hearing mice (chapters 2,3) are in many ways similar to the presynaptic $\mathrm{Ca}^{2+}$ microdomains described in frog (Issa and Hudspeth 1994, Issa and Hudspeth 1996, Zenisek et al. 2003) and turtle hair cells (Tucker and Fettiplace 1995). First, all those studies (as well as this study) note the presence of localized regions of $\mathrm{Ca}^{2+}$ influx (hot-spots). This is consistent with the clustering of VGCCs at presynaptic active zones seen in loose-patch recordings in lower vertebrate hair cells as well as the observation of clusters of intra-membranous particles in freeze-fracture electron microscopy (Roberts et al. 1990). Second, at least for frog hair cells, a presynaptic origin of the fluorescence hot-spots was suggested by their apparent co-localization with synaptic ribbons - although this claim was based on the 'mapping' of light-microscopical images to serial-section electron micrographs (Issa and Hudspeth 1994). Additional evidence for an active zone origin of the fluorescent hot-spots comes from their co-localization with synaptic ribbons in retinal bipolar cells (Zenisek et al. 2004), employing the same ribbon-labeling oligopeptide (binding to ribeye, the major protein of synaptic ribbons) that was used to map co-localization between synaptic ribbons and fluorescent hot-spots in IHCs in this report (chapter 2, Fig. 1B). Again, the initial study on retinal bipolar cells confirmed the correspondence between peptide-labeled structures and synaptic ribbons by electron-microscopy. Third, the good agreement between the voltage-dependencies of fluorescence and whole-cell $\mathrm{I}_{\mathrm{Ca}}$ (Tucker and Fettiplace 1995, Issa and Hudspeth 1996) argues for a lack of substantial contributions of CICR to the observed synaptic $\mathrm{Ca}^{2+}$ signals in lower vertebrate hair cells - agreeing well with the data presented herein (chapter 2, Figs. 2E, 3). Fourth, the degree of spatial confinement of hot-spot signals during (prolonged) stimulation (chapter 2, Figs. 2F, S4) depends on the specific conditions of $\mathrm{Ca}^{2+}$-buffering. Low concentrations of exogenous $\mathrm{Ca}^{2+}$-buffers favor the spread of $\mathrm{Ca}^{2+}$, away from the entry site, as also seen in turtle and frog hair cells (Tucker and Fettiplace 1995, Issa and Hudspeth 1996). Quantitative inter-study comparisons for these aspects are hampered by differences in the amount and type of $\mathrm{Ca}^{2+}$-indicator and $\mathrm{Ca}^{2+}$-buffering used (both exo- and endogenous $\mathrm{Ca}^{2+}$ buffers, Roberts 1993, Hackney et al. 2003, Hackney et al. 2005).

However, the use of spot-detection as compared to line- or frame-scan imaging techniques that were used in the above mentioned studies provides a higher temporal resolution $\approx 2 \mathrm{kHz}$ 
vs. maximal $0.5 \mathrm{kHz}$; Issa and Hudspeth 1996). This improved temporal resolution reveals faster onset kinetics (fast time constant of $\approx 1 \mathrm{~ms}$ (see chapter 2, Fig. 2B, Table S1, and chapter 3, Fig. 5b, Table 2), compared to “( ...) within $6 \mathrm{~ms}$ ", the fastest reported rise in the above mentioned studies; Issa and Hudspeth 1996). Nonetheless, even the fast time constant measured in our spot-detection recordings will underestimate the true speed of $\left[\mathrm{Ca}^{2+}\right]_{\mathrm{i}}$ rise at the active zone, due to spatial averaging over the excitation-detection volume and the limited temporal bandwidth imposed by the kinetics of the $\mathrm{Ca}^{2+}$-indicator reaction (chapter 2, Fig. $\mathrm{S} 1 \mathrm{D}$ and SI text). In comparison, the whole-cell $\mathrm{I}_{\mathrm{Ca}}$ at $-8 \mathrm{mV}$ activates with a time-constant of $360 \mu$ s (chapter 3, Supplementary Fig. 6, in the presence of the gating modifier BayK8644). However, under the conditions used in the imaging experiments $\left(\left[\mathrm{Ca}^{2+}\right]_{\mathrm{e}}=5 \mathrm{mM}\right.$, no BayK), the activation time constant of the whole-cell is likely faster (Neef et al. 2009). In any case, the whole-cell $\mathrm{I}_{\mathrm{Ca}}$ sums over the entire cell and does not provide information about potential local differences in $\mathrm{Ca}^{2+}$ channel activation across synapses (Rodriguez-Contreras and Yamoah 2001) - which might have important implications for the coding of sound onset. To experimentally approach the measurement of the true speed of $\left[\mathrm{Ca}^{2+}\right]_{\mathrm{i}}$ rise at active zones, one may require to decrease the size of the excitation-detection volume (i.e. the point-spread function (PSF) of the imaging system; Shuai and Parker 2005) or use a $\mathrm{Ca}^{2+}$ indicator with a faster association rate $\left(\mathrm{k}_{\mathrm{on}}\right)$. Additionally, one could attempt to de-convolve the measured fluorescence signal with the temporal 'transfer function' of the $\mathrm{Ca}^{2+}$-detection system. However, the reliability of this approach would critically depend on sufficient knowledge about the respective characteristics of the $\mathrm{Ca}^{2+}$-detection system (kinetics of $\mathrm{Ca}^{2+}$ indicator and nonfluorescent buffer, PSF dimension, and its localization with respect to the $\mathrm{Ca}^{2+}$ source).

This study shows for the first time that the fluorescence hot-spots can also be observed under close-to-native $\mathrm{Ca}^{2+}$-buffering conditions (in the presence of at least $80 \%$ of the initial levels of the three main proteinaceous $\mathrm{Ca}^{2+}$ buffers of IHCs; chapter 2, SI text). Kinetically, the experiments in the presence of endogenous $\mathrm{Ca}^{2+}$ buffers were most comparable to those in which the intracellular mobile buffer was $0.5 \mathrm{mM}$ EGTA (chapter 2, Fig. 4). Differences in the association and dissociation kinetics of $\mathrm{Ca}^{2+}$ binding between the three endogenous buffers as well as their strongly varying degrees of cooperativity in $\mathrm{Ca}^{2+}$ binding (Nagerl et al. 2000, Faas et al. 2007) complicate simple comparisons of their effects to the effect of exogenously added EGTA. Nonetheless, it is interesting to note that the concentration of binding sites was estimated to lie in the range of $0.5 \mathrm{mM}$ for mature rodent IHCs (Hackney et al. 2005). 
While this description of $\mathrm{Ca}^{2+}$ microdomain signals in mature mouse IHCs agrees well with and extends - observations made in hair cells of lower vertebrates, it differs in a number of aspects from an initial study on $\mathrm{Ca}^{2+}$ microdomains in immature mouse IHCs (Kennedy and Meech 2002). First, Kennedy and Meech reported a spatially more homogenous rise in $\left[\mathrm{Ca}^{2+}\right]_{\mathrm{i}}$, as opposed to a clear localization of (high) $\mathrm{Ca}^{2+}$ influx seen in the form of fluorescence hot-spots. One possible explanation for this discrepancy is the different developmental stage of IHCs under study. It is known that many conductances in rodent IHCs, including $\mathrm{Ca}_{\mathrm{v}} 1.3$ channels, undergo substantial up/down-regulations in strength between the terminal mitosis (at around embryonic day (E)14-15) and the onset of hearing (at around postnatal day (P)12; reviewed in: Housley et al. 2006). The initial up-regulation of whole-cell $\mathrm{Ca}^{2+}$ conductance peaks at around P6-7 and is followed by a significant down-regulation to the levels found in mature IHCs after the onset of hearing (Beutner and Moser 2001). Recently it has been shown by immuno-histochemistry that at the developmental stage of peak $\mathrm{Ca}^{2+}$ conductance, the number of $\mathrm{Ca}^{2+}$ channel clusters is several-fold larger than in mature IHCs (Zampini et al. 2010). The observed presence of $\mathrm{Ca}_{\vee} 1.3$ clusters, however, renders a homogenous density of $\mathrm{Ca}^{2+}$ influx throughout the plasma membrane of the basal pole of IHCs unlikely, although the morphological presence of such clusters may not inevitably mirror the localization of physiologically active $\mathrm{Ca}^{2+}$ channels. Another likely reason for the disparities in the observed spatial $\mathrm{Ca}^{2+}$ profiles is the use of a high-affinity dye (Fluo-3; $\mathrm{K}_{\mathrm{D}} \approx 500 \mathrm{nM}$, Naraghi 1997). High-affinity dyes do not necessarily report the true spatial gradients of $\left[\mathrm{Ca}^{2+}\right]_{\mathrm{i}}$, as their low $\mathrm{K}_{\mathrm{D}}$ likely leads to their quick saturation in the vicinity of $\mathrm{Ca}^{2+}$ entry sites. Besides its low $\mathrm{K}_{\mathrm{D}}$, Fluo-3 exhibits fast kinetics of binding and unbinding, similar to the 'fast' $\mathrm{Ca}^{2+}$-chelator BAPTA (Naraghi 1997). As a consequence, fluo-3 can effectively 'shuttle' $\mathrm{Ca}^{2+}$ ions and thus facilitate their diffusion (Dargan and Parker 2003), further 'globalizing' the observed $\mathrm{Ca}^{2+}$ signals. This especially holds true if comparably low concentrations of additional buffers that could spatially constrain the $\left[\mathrm{Ca}^{2+}\right]_{\mathrm{i}}$ elevations - are present $\left(1 \mathrm{mM}[\mathrm{EGTA}]_{\mathrm{i}}\right.$, Kennedy and Meech 2002). However, the developmental changes in $\mathrm{Ca}^{2+}$ conductance are also accompanied by a functional 'maturation' of the synaptic neurotransmitter release machinery. This maturation is characterized by a switch from a supra-linear (before the onset of hearing) to a more linear relationship (after the onset of hearing) between $\mathrm{Ca}^{2+}$ influx and exocytosis (Beutner and Moser 2001, Johnson et al. 2005). Although the exact mechanisms behind this shift have not been determined, the local $\left[\mathrm{Ca}^{2+}\right]_{\text {i }}$ profiles in the vicinity of the release-ready synaptic vesicles at the active zones likely play a critical role. Yet, the notion that - unlike in 
mature IHCs - a considerable fraction of $\mathrm{Ca}_{\mathrm{V}} 1.3$ clusters does not co-localize with synaptic ribbons in immature IHCs, might explain part of that linearization of $\mathrm{Ca}^{2+}$-dependence of exocytosis seen in mature IHCs (Zampini et al. 2010). In light of these developmental differences in $\mathrm{Ca}^{2+}$ channel organization and function, the observation of a more homogenous $\left[\mathrm{Ca}^{2+}\right]_{\mathrm{i}}$ rise in immature IHCs may also reflect a (partly) different functional role of IHC Cav1.3 channels in early development, such as a 'purely electrical' one $\left(\mathrm{Ca}^{2+}\right.$ action potential generation; Tritsch and Bergles 2010).

Second, the absence of evidence for $\mathrm{Ca}^{2+}$-induced $\mathrm{Ca}^{2+}$ release (CICR) (chapter 2, Figs. 3B-D, $\mathrm{S} 3$, Tables S1, S2) is in contrast to the reported requirement of CICR for the fast component of the $\left[\mathrm{Ca}^{2+}\right]_{\mathrm{i}}$ rise in immature IHCs (Kennedy and Meech 2002). This discrepancy could, again, simply reflect a differential importance of CICR at different developmental stages (immature vs. hearing). Interestingly, however, CICR has also been implied in regulation of afferent neurotransmission in vestibular hair cells (Lelli et al. 2003). An augmentation of the hair cell $\left[\mathrm{Ca}^{2+}\right]_{\mathrm{i}}$ signal was seen for long $(500 \mathrm{~ms})$, but not for short $(50 \mathrm{~ms})$ depolarizations. Moreover, the sites at which maximal CICR-caused $\left[\mathrm{Ca}^{2+}\right]_{i}$ increases were observed, were distinct from the sites of depolarization-induced fluorescence hot-spots. Thus, the lack of observing CICR in the present study could be due to the short stimuli used ( $20 \mathrm{~ms}$ depolarizations) and the resulting insufficient $\left[\mathrm{Ca}^{2+}\right]_{i}$ at the remote sites where intracellular stores are equipped with ryanodine receptors. Alternatively, the operation of IHC synapses at high frequencies - reflected in the observation of phase-locking in the postsynaptic auditory nerve fibers for frequencies up to $4 \mathrm{kHz}$ (mice; Taberner and Liberman 2005) - may oppose the implementation of a temporally and spatially less tightly controlled process such as CICR in the regulation of neurotransmitter release. Additionally, CICR could also adversely affect the precise coding of lower frequencies. Mature rodent IHCs do, however, express ryanodine receptors - as suggested by an immuno-electron-microscopy study in rats (Grant et al. 2006). Labeling in IHCs was most dense (i) on subsurface cisternae located beneath the basolateral membrane, in the region between the nucleus und the apical pole of the IHCs, and (ii) on rough endoplasmic reticulum between the base of the IHC and the nucleus. The latter region was located approximately $0.5 \mu \mathrm{m}$ from the nearest afferent terminal, and only sparse staining, not clearly related to specific structures, was observed closer to afferent synapses. Although, at this point, we have no positive evidence for a role of CICR in regulation of afferent neurotransmission in mature mouse IHCs, we cannot ultimately exclude this possibility, perhaps also utilizing inositol trisphosphate- (IP3-) sensitive stores. If present, for example dur- 
ing long and intense stimulation, it will be interesting to address the physiological significance of such a potential CICR contribution.

In contrast to immature mouse IHCs, for which some contribution of mitochondria to "shortterm' $\mathrm{Ca}^{2+}$ homeostasis was reported (Kennedy 2002), we did not find evidence for a strong effect of mitochondrial $\mathrm{Ca}^{2+}$ uptake on $\mathrm{Ca}^{2+}$ microdomain signals at mature IHC ribbon synapses (chapter 2, Fig. 3D, Table S1). This observation could reflect differences in the developmental stage, as - due to their large $\mathrm{Ca}^{2+}$ conductance - immature mouse IHCs have to bear with large $\mathrm{Ca}^{2+}$ loads, especially during bursts of $\mathrm{Ca}^{2+}$-carried action potentials (Beutner and Moser 2001, Tritsch et al. 2007). Alternatively, a rather modest effect of mitochondrial $\mathrm{Ca}^{2+}$ uptake may have escaped our detection, as we used a less sensitive dye (Fluo-4FF, $\mathrm{K}_{\mathrm{D}} \approx$ $10 \mu \mathrm{M}$, as compared to Fluo-3; see above). However, despite adjusting our $\mathrm{Ca}^{2+}$-detection configuration (using Fluo-4FF and entailing weak cytosolic buffering (0.5 mM EGTA)) we did not find evidence for mitochondrial $\mathrm{Ca}^{2+}$ uptake - even during prolonged $\mathrm{Ca}^{2+}$ influx (150 $\mathrm{ms})$. This is in line with reports from retinal bipolar neurons, in which the primary role of mitochondria at ribbon synapse terminals was found to be ATP production rather than $\mathrm{Ca}^{2+}$ handling (Zenisek and Matthews 2000).

\subsubsection{Heterogeneity of $\mathrm{Ca}^{2+}$ Microdomains}

How does the herein reported $\mathrm{Ca}^{2+}$ microdomain amplitude heterogeneity relate to the scatter of microdomain amplitudes observed in lower vertebrate hair cells? Using focal current recordings (loose-patch recordings) at the basolateral membrane of frog saccular hair cells, Roberts and colleagues reported a more than ten-fold variation in the $\mathrm{Ca}^{2+}$ current density (Roberts et al. 1990). Moreover, the same study describes the observation of intra-membranous particles at active zones in freeze-fracture electron micrographs. Although there is no direct evidence that these particles actually correspond to ion channels, the very good agreement between the average particle number per active zone $(\approx 130)$ and the independently inferred average numbers per active zone for $\mathrm{Ca}^{2+}(\approx 90)$ and $\mathrm{Ca}^{2+}$-activated $\mathrm{K}^{+}$channel numbers $(\approx 40)$ - that co-localize in clusters at these active zones (Roberts et al. 1990) - suggests that those particles could correspond to these ion channels. The number of these particles was variable between active zones, with 4-fold differences between the smallest and the largest count (table 6, Roberts et al. 1990). The coefficient of variation (CV) in particle counts was 0.41 , slightly smaller than our most tightly controlled estimate of 0.54 for $\mathrm{Ca}^{2+}$ microdomain variability in mouse IHCs (chapter 2, SI text). 
Using cell-attached recordings, another study noted the presence of $\mathrm{Ca}^{2+}$ channels clusters with different channel densities in frog saccular hair cells (Rodriguez-Contreras and Yamoah 2001). This observation is reflected in a heavily positively skewed distribution of $\mathrm{Ca}^{2+}$ channel densities (Fig. 11, Rodriguez-Contreras and Yamoah 2001). Beyond the graphical representation, however, no further quantification of the statistical dispersion of the sample population is mentioned. It is important to note that this type of hair cells seemingly expresses not only L-type channels, but at least one more class of non L-type $\mathrm{Ca}^{2+}$ channels (RodriguezContreras and Yamoah 2001). Yet, the authors report that the two types of $\mathrm{Ca}^{2+}$ channels colocalized in only $6 \%$ of the measured membrane patches.

Most $\mathrm{Ca}^{2+}$ imaging studies performed in hair cells, on the other hand, do not report on the quantification of large numbers of $\mathrm{Ca}^{2+}$ signal amplitudes. This shortage of available data somewhat precludes the comparison of the heterogeneity of $\mathrm{Ca}^{2+}$ microdomains investigated in this study with optical recordings of $\mathrm{Ca}^{2+}$ microdomain signals in immature IHCs or lower vertebrate hair cells. One exception is the report of average $\mathrm{Ca}^{2+}$ concentrations within circular regions (of $2 \mu \mathrm{m}$ diameter) overlying the respective hot-spots (Tucker and Fettiplace 1995). A CV of 0.82 was seen for the average $\mathrm{Ca}^{2+}$ concentrations of 21 hot-spots. However, the same study reports on a CV of 0.45 for 3 hot-spots, when using smaller measurement windows and an objective lens with higher numerical aperture. It is important to note that in both cases, the $\mathrm{Ca}^{2+}$ concentration values were estimated at the end of long depolarizations ( 300 to $500 \mathrm{~ms}$ ), and, thus, could be shaped by mechanisms like $\mathrm{Ca}^{2+}$ extrusion and sequestration, found to affect these $\mathrm{Ca}^{2+}$ signals on that time-scale (same study). Another study noted that L-type $\mathrm{Ca}^{2+}$ channels in frog semicircular canal hair cells appeared in different densities across different hot-spots (Rispoli et al. 2001). This conclusion is based on the observation of different degrees of signal attenuation seen under focal application of the L-type-channel blocker nifedipine to individual hot-spots. However, unlike in $\mathrm{IHCs}$, the $\mathrm{Ca}^{2+}$ current in these hair cells appears to be more diverse, not only being carried by L-type channels, but additionally by two types of R-type channels (Martini et al. 2000). Last, it is interesting to note that a study in turtle hair cells speculated about the 'quantization' of $\mathrm{Ca}^{2+}$ microdomain signal amplitudes based on a correlation between the prevalence of peak amplitude multiples with the choice of large regions for hot-spot analysis (potentially encompassing multiple sites of $\mathrm{Ca}^{2+}$ entry; Ricci et al. 2000). By comparing $\mathrm{Ca}^{2+}$ microdomain area and number, as well as wholecell $\mathrm{Ca}^{2+}$ channel number between two different frequency positions (see also section 4.5), the authors moreover suggest that $\mathrm{Ca}^{2+}$ channels are present at constant density between the dif- 
ferent tonotopic locations. However, the use of the high-affinity indicator Calcium-Green 1 (as dextran-conjugate) in that study may have affected amplitude and area estimation of the $\mathrm{Ca}^{2+}$ microdomains. Indeed, a later study suggested significant differences in the magnitude of synaptic $\mathrm{Ca}^{2+}$ influx between low- and high-frequency turtle hair cells - however based on relating morphological assessment of synapse number to whole-cell $\mathrm{I}_{\mathrm{Ca}}$ amplitude (Schnee et al. 2005; see below).

What are the consequences of the observed heterogeneity among individual $\mathrm{Ca}^{2+}$ microdomains for the firing characteristics of the postsynaptic targets of these non-mammalian hair cells? Compared to the amount of data available on mammalian ANFs, however, little is known about the postsynaptic neurons in these lower vertebrates. It is thus unclear whether the presynaptic $\mathrm{Ca}^{2+}$ signal variability seen in hair cells is accompanied by variations in the firing of postsynaptic neurons. In a recent study, spiking activity was recorded from single VIIIth cranial nerve fibers in the frog, postsynaptic to saccular hair cells (Rutherford and Roberts 2009). While the authors report pronounced differences in the recorded postsynaptic activities, ranging from complete absence of spiking to various kinds of 'bursting' behavior, it is unclear, to which degree this range of firing characteristics reflects physiological or pathological behavior due to damage caused during the preparation (Rutherford and Roberts 2009). The interpretation is further complicated by the fact that the neurites that were targeted in these recordings contact multiple hair cells (Rutherford and Roberts 2009). Interestingly, some degree of variation was also found in the synaptic transfer function between different pairs of hair cells and afferent fibers in the amphibian papilla, the principal auditory receptor organ of frogs (Keen and Hudspeth 2006).

\subsection{Potential Artefacts in $\mathrm{Ca}^{2+}$ Microdomain Amplitude Estimation}

While the optical measurement of $\mathrm{Ca}^{2+}$ signals has obvious advantages over classical electrophysiological recordings of $\mathrm{Ca}^{2+}$ influx (see chapter 1.3), it also bears some disadvantages which have to be considered when interpreting $\mathrm{Ca}^{2+}$ imaging data. First, unlike voltage-clamp techniques that directly measure the flow of ions across the plasma membrane, $\mathrm{Ca}^{2+}$ indicators signal changes in $\left[\mathrm{Ca}^{2+}\right]$ by an alteration of their photophysical properties upon $\mathrm{Ca}^{2+}$ binding (see chapter 1.3). Thus, the required $\mathrm{Ca}^{2+}$ binding step introduces a temporal delay in the detection of $\mathrm{Ca}^{2+}$ influx and low-pass filters the signal - with the exact response characteristics determined by both the on- and off-rates of the $\mathrm{Ca}^{2+}$-indicator reaction. However, at the same 
time, $\mathrm{Ca}^{2+}$ indicators are often highly specific over other cations, at least compared to monovalent species such as $\mathrm{Na}^{+}$and $\mathrm{K}^{+}$, allowing discrimination of $\mathrm{Ca}^{2+}$ flux from other ion fluxes without the need to use specific ion channel blockers (chapter 2, Fig. 3).

The advantage of spatial selectivity in imaging-based measurements of $\left[\mathrm{Ca}^{2+}\right]_{\mathrm{i}}$ at the same time poses the need of cautious interpretation of these data. This is because any measured signal will be a function of the true, underlying $\left[\mathrm{Ca}^{2+}\right]_{i}$ and the exact spatial relationship between this signal and the respective detector. For instance, in a laser-scanning confocal microscope (LSCM), the light from a point source (e.g. a single-mode laser beam) is focused into a single diffraction-limited spot in the object plane. If this diffraction image of the point-source is brought in good register with the $\mathrm{Ca}^{2+}$ influx site, the small size of the conjugate detection volume (the illuminated volume in the field of view of the pinhole-'shielded' detector) ensures a very good signal-to-background ratio for fluorescence changes (large dynamic range of changes in $\mathrm{Ca}^{2+}$-bound indicator concentration), and at the same time a high temporal resolution (as the reaction and diffusion processes underlying the fluorescence changes within the volume quickly approach a steady-state; see also: Shuai and Parker 2005). However, deviations in the localization of the excitation-detection volume with respect to the $\mathrm{Ca}^{2+}$ signal source will accordingly lead to an underestimation of true signal amplitude, as less incoming $\mathrm{Ca}^{2+}$ ions are actually being sampled by the detector, and a slowing in the observed response kinetics (reflecting the impact of longer diffusion times between the source and the excitationdetection volume). To account for this problem in our experiments (chapter 2, Fig. 2B, SI text), we routinely recorded at seven adjacent positions, spaced left and right of the beforehand identified $\mathrm{Ca}^{2+}$ microdomain (chapter 2, Fig. 2A), and afterwards restricted analysis to the maximum intensity trace, presumably reflecting the signal from or closest to the center of the $\mathrm{Ca}^{2+}$ microdomain. Although the excitation-detection volume was found to be $>4$-fold larger in the axial $(Z)$ as compared to the lateral $(X, Y)$ dimension $\left(\mathrm{FWHM}_{\mathrm{Z}} \approx 1.25 \mu \mathrm{m}\right.$, $\mathrm{FWHM}_{\mathrm{X}, \mathrm{Y}} \approx 0.30 \mu \mathrm{m}$; chapter 2 , Table S3) and alignment along the optical axis $(Z)$ is consequently expected to be less critical, we also routinely attempted to axially register signal source and detection volume. Preceding spot detection recordings, we thus (manually) compared the intensity of the same $\mathrm{Ca}^{2+}$ microdomain in optical sections above and below the focal plane of its initial identification, in order to best match the plane of subsequent acquisition to the center plane of the $\mathrm{Ca}^{2+}$ microdomain. 
We nonetheless tried to estimate to which degree the heterogeneity in the actually analyzed $\mathrm{Ca}^{2+}$ microdomain amplitudes may still have arisen from suboptimal alignment of excitationdetection volume and $\mathrm{Ca}^{2+}$ influx site. As outlined earlier, a smaller overlap between the two predicts a slowing in the kinetics, and a decrease in the amplitude of the responses. We therefore compared the onset kinetics of $\mathrm{Ca}^{2+}$ microdomain signals with their corresponding amplitude in a sample of $65 \mathrm{Ca}^{2+}$ microdomains and found a weak, positive correlation between the fast onset time constant and the amplitude (chapter 3, Fig. 6c). In contrast, a negative correlation would have been expected for a misalignment between $\mathrm{Ca}^{2+}$ influx site and the excitation-detection volume. We further addressed the issue of potential alignment errors in experiments that attempted to minimize them by systematic identification of the $\mathrm{Ca}^{2+}$ microdomain center in $\mathrm{X} / \mathrm{Y} / \mathrm{Z}$ by '3D spot displacement' (chapter 2, SI text). Although a reduction in the amplitude heterogeneity was observed in these experiments, the $\mathrm{Ca}^{2+}$ microdomain amplitudes still showed considerable scatter $(\mathrm{CV}=0.54)$. Further evidence for a certain degree of experimentally introduced variability comes from line-scan experiments that were usually performed on $\mathrm{Ca}^{2+}$ microdomains after spot-detection measurements (chapter 2, Fig. 2F; chapter 3, Fig. 5d). In a data set of 87 line-scan measurements, we find a weak, negative correlation between amplitude and FWHM of the $\mathrm{Ca}^{2+}$ microdomains (chapter 3; Fig. 6d) - consistent with the idea of a slight axial mismatch between the $\mathrm{Ca}^{2+}$ influx site and the focal plane that results in an image of the object that is broader and has reduced peak intensity upon defocusing. However, also a biological explanation of the observed correlation cannot be ruled out (see below; Fig. A1C). In summary, although some of the observed variance seemingly is due to experimental reasons, its largest fraction appears to reflect other sources.

Another, related, uncertainty is connected to the fact that the exact orientation of the $\mathrm{Ca}^{2+} \mathrm{mi}$ crodomain signal with respect to the excitation-detection volume is not known. Modeling indicated that immuno-labeled $\mathrm{Ca}_{\mathrm{V}} 1.3$ clusters that were imaged by STED microscopy could be well described by flat, oblate spheroids (chapter 3, Fig. 3g, Fig. S5). Hence, we assume that the real $\mathrm{Ca}_{\mathrm{V}} 1.3$ clusters are circular in the plane of the plasma membrane. Two extreme cases can hence be considered for our $\mathrm{Ca}^{2+}$ imaging experiments: one in which the plasma membrane (with the $\mathrm{Ca}_{\mathrm{V}} 1.3$ cluster) is oriented orthogonal to the optical axis, and one in which it is oriented parallel to it (Fig. A1A). Due to the impermeability of the plasma membrane to $\mathrm{Ca}^{2+}$ ions it is clear that imaging will give different results in the two cases. The question is, to which degree these two (limit case) scenarios differ in their measured $\mathrm{Ca}^{2+}$ microdomain amplitude. As the exact orientation of a given $\mathrm{Ca}^{2+}$ microdomain is inaccessible to experimental 
determination at the time of imaging, we used the $\mathrm{Ca}^{2+}$ reaction-diffusion-imaging model to assess the impact of $\mathrm{Ca}_{\mathrm{V}} 1.3$ cluster orientation with respect to the optical axis of the imaging system (Fig. A1A). As intuitively expected, the simulation suggests that the signal is larger under parallel register of the plasma membrane and the optical axis, due to the fact that a larger region of the microdomain is covered by the excitation-detection volume. The simulation predicts a difference of about $40 \%$ in the measured fluorescence change between the two scenarios (Fig. A1B). To estimate the impact of this measurement-related amplitude variability we constructed a model amplitude distribution (randomly drawing numbers from 1 to 10 at equal probability, to account for the maximally observed amplitude range of $\approx 10$; see chapter Fig. 6b). While this model distribution had a $\mathrm{CV}$ of $\approx 0.47$, the random multiplication of its entries with a number between $\approx 0.6$ and 1 to simulate imaging-related amplitude differences of $40 \%$ to $0 \%$ (again from a continuous uniform probability distribution), resulted in a CV of $\approx 0.48$. While intuitively expected, this supports the notion that if orientation and amplitude of $\mathrm{Ca}^{2+}$ microdomains are two independent parameters, the overall variance will be largely determined by the amplitude heterogeneity. At the same time, the reaction-diffusion-imaging simulations predict broader microdomains with lower amplitude for the orthogonal vs. parallel arrangement (Fig. A1C), which is interesting in light of the observation of such negative correlation found in actual line-scan experiments (see above; chapter 3, Fig. 6d).

Additionally, unwanted variability in the $\mathrm{Ca}^{2+}$ microdomain population could arise from differences in the whole-cell $\mathrm{Ca}^{2+}$ influx - between cells and within one cell over the time course of an experiment. Several lines of evidence, however, render this possibility unlikely to be of major contribution to the observed amplitude heterogeneity. First, we find large (up to $>10$ fold) amplitude variability within single IHCs, and the average ratio of maximum/minimum $\mathrm{Ca}^{2+}$ microdomain amplitude within cells was 4.5 (chapter 3, Fig. 6b). Additionally, the average intracellular $\mathrm{CV}$ (calculated for IHCs in which at least $3 \mathrm{Ca}^{2+}$ microdomains were recorded) was 0.57 , very close to the $\mathrm{CV}$ of the entire population (chapter 3, Fig. 6). Very similar results were also seen in a second sample (chapter 2, Fig. S2). The notion of a population heterogeneity that is largely determined by intracellular variability is further supported by the similarity of the bootstrap distributions of $\mathrm{Ca}^{2+}$ microdomain ratios drawn from either purely intracellular or purely intercellular $\mathrm{Ca}^{2+}$ microdomain distributions (chapter 2, Figs. S2c, d, SI text). Second, the amplitude of the $\mathrm{Ca}^{2+}$ microdomain signals did not change systematically over time, and large variance was observed throughout the time course of experiments (chapter 3, Fig. 6b), arguing against $\mathrm{Ca}^{2+}$ current rundown being a significant factor in determining 
the amplitude heterogeneity. This notion is further strengthened by the observation, that 'normalizing' the $\mathrm{Ca}^{2+}$ microdomain amplitudes to the corresponding, simultaneously acquired whole-cell $\mathrm{Ca}^{2+}$ current did not decrease the population $\mathrm{CV}$ (Figs. A2A, B). Finally, we have no evidence for a significant variance contribution of the imaging system. This is reflected in the very low $\mathrm{CV}$ of 0.02 in a data set comprising 39 spot detection measurements performed on a bath of fluorescein solution $(100 \mu \mathrm{M})$, at a laser intensity setting set to match the observed fluorescence to the $\mathrm{Ca}^{2+}$ microdomain amplitude (Fig. A2C). In summary, We demonstrate that the observed heterogeneity reflects large differences in $\mathrm{Ca}^{2+}$ microdomain amplitude within and between cells. Experimental variance from uncertain positioning of the confocal spot and $\mathrm{Ca}^{2+}$ current run-down cannot explain the large $\mathrm{CV}$ observed.

\subsection{Mechanisms Contributing to $\mathrm{Ca}^{2+}$ Microdomain Heterogeneity}

The large heterogeneity in $\mathrm{Ca}^{2+}$ microdomain amplitudes naturally poses the question of underlying mechanism(s). We have gathered several pieces of indirect evidence for differences in $\mathrm{Ca}^{2+}$ channel number between IHC presynaptic active zones. First, immuno-labeling indicated inter-synaptic differences in the $\mathrm{Ca}_{\mathrm{V}} 1.3$ antigen-content within a large sample of synapses (chapter 2, Fig. 5E). However, this approach is afflicted with some uncertainties. For example, there could be potential differences in antigen accessibility between synapses or even within a given $\mathrm{Ca}_{\mathrm{V}} 1.3$ cluster. If antigen accessibility posed a problem - as reported for voltage-gated sodium channels in the axon initial segment (Lorincz and Nusser 2008) - it should falsify the conclusions only if there were systematic differences across synapses, which by itself still could represent an interesting observation. Yet, the use of methanol, a denaturing fixative, may have alleviated potential epitope-masking by cross-linking $\mathrm{Ca}_{\mathrm{V}} 1.3$ with its binding partners. Nonetheless, in order to amend the immuno-labeling approach and directly test the hypothesis of an impact of $\mathrm{Ca}^{2+}$ channel complement on the respective $\mathrm{Ca}^{2+}$ microdomain signals, we used the rhodamine-conjugated ribeye-labeling peptide to semiquantitatively assess the number of binding sites (i.e. the number of ribeye molecules and hence, most likely ribbon size; Zenisek et al. 2004) within a given synaptic ribbon during the recording (chapter 2, Fig 1B, Fig. 5B). This peptide signal was positively correlated to the subsequently in-place determined $\mathrm{Ca}^{2+}$ microdomain amplitude (chapter 2, Fig 5D). This suggests that - at least on average - the presence of a larger ribbon coincides with larger $\mathrm{Ca}^{2+}$ influx at the active zone, consistent with positive correlations between ribbon size, release are 
and $\mathrm{Ca}^{2+}$ channel number found in various other hair cell types (Martinez-Dunst et al. 1997). Last, also the size distribution of immuno-labeled $\mathrm{Ca}_{\mathrm{V}} 1.3$ cluster in IHCs - as determined by STED microscopy - covered a large range of values (CV of 0.3 for $\mathrm{Ca}_{\mathrm{V}}$ diameter; chapter 3 , Fig. 3g). However, no correlation with functional $\mathrm{Ca}^{2+}$ imaging data was possible. In summary, the currently available data argue for differences in $\mathrm{Ca}^{2+}$ channel number as one main source of the observed heterogeneity in $\mathrm{Ca}^{2+}$ microdomain amplitude. It would be interesting to further study, for instance by STED-based $\mathrm{Ca}^{2+}$ imaging, whether differences between synapses also exist in terms of Cav1.3 density, as the channel density likely has important implications for the coupling of $\mathrm{Ca}^{2+}$ influx to transmitter release. Modeling predicts that the current imaging setup is not very sensitive to differences in the density of $\mathrm{Ca}^{2+}$ influx (Figure A3), but rather to its absolute flux amplitude.

Besides variations in $\mathrm{Ca}^{2+}$ channel number, synapses could also exhibit differences in $\mathrm{Ca} 2+$ channel gating. When uniformly elevating the open probability of the $\mathrm{Ca}^{2+}$ channels by application of the dihydropyridine agonist BayK8644 (Hess et al. 1984) - in an attempt to equalize potential differences in open probability across synapses - we, however, did not find an effect on $\mathrm{Ca}^{2+}$ microdomain amplitude variability (chapter 2, Table S1). Yet, the absence of such an effect does not rule out the presence of differences in gating between sub-populations of IHC $\mathrm{Ca}^{2+}$ channels and requires further investigation. In contrast to other L-type channels, especially $\mathrm{Ca}_{\mathrm{v}} 1.2$, little is known, however, about whether and how the gating of Cav1.3 channels is regulated by subunit expression and/or protein modification. Only recently it was shown that genetic interference with the main $\beta$-subunit of VGCCs in IHCs $\left(\mathrm{Ca}_{\mathrm{V}} \beta_{2}\right)$ severely reduced the level of $\mathrm{Ca}_{\mathrm{V}} 1.3$ channels in the plasma membrane of IHCs, without, however, affecting the gating of the remaining channels (Neef et al. 2009). Notably, although not systematically studied, the variability of $\mathrm{Ca}^{2+}$ microdomain amplitudes seemed to be reduced in these $\mathrm{Ca}_{\mathrm{V}} \beta_{2}$ knockout animals, further highlighting the relationship between synaptic $\mathrm{Ca}^{2+}$ channel complement and $\mathrm{Ca}^{2+}$ microdomain amplitude heterogeneity (see above). One very extensively studied mechanism of L-type channel regulation is the phosphorylation of the $\alpha_{1}$-subunit that happens at several distinct sites in $\mathrm{Ca}_{\mathrm{V}} 1.2$ channels. This modification may have very different effects on channel function, depending on the phosphorylation site, the type of kinase involved, and the exact cell type under study (Dai et al. 2009). Much less is known about phosphorylation of $\mathrm{Ca}_{\mathrm{V}} 1.3$ channels, and even less about to which degree this type of regulation is important in IHCs. Protein kinase C (PKC) phosphorylation has been shown to inhibit $\mathrm{Ca}_{\mathrm{V}} 1.3$ currents in a heterologous expression system, but whether these results can be translated to 
native $\mathrm{Ca}_{\mathrm{V}} 1.3$ channels in IHCs remains unclear (Baroudi et al. 2006). Another study reported an augmenting effect of protein kinase $\mathrm{A}$ (PKA) on a subset of $\mathrm{Ca}_{\mathrm{V}} 1.3$ currents in HEK cells (Liang and Tavalin 2007). Interestingly, the effect depended on the type of $\beta$-subunit coexpressed. Last, an immuno-histochemical investigation has provided evidence for the expression of the $\mathrm{Ca}^{2+}$-regulated protein phosphatase calcineurin (protein phosphatase $2 \mathrm{~B}$ or protein phosphatase 3) in IHCs of the rat (Kumagami et al. 1999). Notably, a spot-like pattern of immuno-labeling was reported in that study, however the exact sub-cellular location of the antigen remained unclear, as did the functional significance of this finding especially with respect to a potential regulation of $\mathrm{Ca}_{\mathrm{V}} 1.3$ channels.

Besides large heterogeneity in the amplitudes across different $\mathrm{Ca}^{2+}$ microdomains, we also found considerable variability in their voltage-dependence of activation (chapter 2, Fig. 5A). While this observation cannot account for the differences seen in $\mathrm{Ca}^{2+}$ microdomain amplitudes, as the data used for amplitude comparison were all acquired at a membrane potential of $-7 \mathrm{mV}$, where activation of whole-cell $\mathrm{Ca}^{2+}$ current and synaptic $\mathrm{Ca}^{2+}$ influx saturates (chapter 2, Fig. 5A), it presents an interesting finding by itself. Ruling out or correcting for potential artifacts such as drift of electrode potential, and voltage drop over the series resistance, we note that $\mathrm{Ca}^{2+}$ microdomains still differ in their voltage-dependence of activation, most interestingly even across active zones of an individual IHC. As the hair cell is isopotential (Roberts et al. 1990), we also exclude space-clamp errors as a potential artifact. Reactiondiffusion-imaging simulations suggest that the observed voltage-dependence of the $\mathrm{Ca}^{2+}$ microdomain signal is invariant to the exact spatial relationship between the excitation-detection volume and the $\mathrm{Ca}^{2+}$ microdomain (Fig. A4). Variations in the goodness of co-localization between the confocal spot and the $\mathrm{Ca}^{2+}$ influx site across different $\mathrm{Ca}^{2+}$ microdomains are hence unlikely to account for the observed heterogeneity in voltage-dependence. Functionally, these differences between active zones could explain the observation of different FM1-43 destaining thresholds in guinea pig IHCs (Griesinger et al. 2005), however the exact nature of those fluorescent signals is under debate (see below).

What could be the origin of the intra-cell variability in $\mathrm{Ca}^{2+}$ microdomain voltage-dependence of activation? The fact that more than $90 \%$ of the $\mathrm{Ca}^{2+}$ current in mature IHCs is carried by $\mathrm{Ca}_{\vee} 1.3$ channels (Brandt et al. 2003) argues against a differential distribution of channel types (e.g. $\mathrm{Ca}_{\mathrm{V}} 1.4$ ) across active zones as the cause of heterogeneity in their voltage-dependence of activation. It is, however, interesting to note, that two splice variants of the Cav1.3- $\left(\alpha_{1} D\right)$ - 
subunit are expressed in the mouse cochlea (Singh et al. 2008). These two splice variants differ in their activation kinetics. When co-expressed with $\beta_{3}$ - and $\alpha_{2} \delta$-subunits in HEK cells, the longer isoform (full-length; $\mathrm{Ca}_{\mathrm{V}} 1.3_{42}$ ) shows sizeable activation at more positive voltages than the shorter isoform ( $\mathrm{Ca}_{\mathrm{V}} 1.3_{42 \mathrm{~A}}$; lacking a C-terminal modulator sequence; Singh et al. 2006). This difference in voltage-dependence of activation is reflected in a shift of approximately 10 $\mathrm{mV}$ in the voltage of half-activation $\left(\mathrm{V}_{1 / 2}\right)$. Seemingly, the threshold of activation is similar between the two $\mathrm{Ca}^{2+}$ channels, while the $\mathrm{Ca}_{V} 1.3_{42 \mathrm{~A}}$ isoform shows a smaller slope factor. Notably, a substantially higher $(\approx 2.5$-fold $)$ current density - albeit an apparently similar expression density - was reported for cells expressing the truncated isoform (Singh et al. 2008). In light of this finding, it is interesting to note that we did not find a correlation between the voltage of half-activation and the $\mathrm{Ca}^{2+}$ microdomain amplitude. Recent single-channel recordings from immature mouse IHCs moreover detected only a single population of $\mathrm{Ca}^{2+}$ channels in the basolateral membrane (Zampini et al. 2010). However, the observation of maximally 4 simultaneously active channels in one patch contrasts with earlier reports on recordings from patches with large numbers of simultaneously active $\mathrm{Ca}^{2+}$ channels in frog hair cells (up to 80; Rodriguez-Contreras and Yamoah 2001). This discrepancy was interpreted as a failure to completely remove the afferent terminals from the presynaptic membrane, possibly hindering access to synaptic $\mathrm{Ca}^{2+}$ channels (Zampini et al. 2010). Thus, the reported observations may not be fully representative of all $\mathrm{IHC} \mathrm{Ca}^{2+}$ channels; especially may they miss on a distinct population of synaptic $\mathrm{Ca}^{2+}$ channels, speculated to exist in hair cells (Rodriguez-Contreras and Yamoah 2001). It is currently unclear, however, to which extent the two splice variants are natively expressed in IHCs, and whether the observed differences in gating persist in the specific subunit-environment of IHCs (e.g. $\beta_{2}$ - instead of $\beta_{3}$-subunit; Neef et al. 2009). Yet, hypothetically, a mosaic-like expression pattern in single IHCs could equip active zones with varying ratios of the two splice variants, leading to differences in the 'compound' voltagedependence of activation of the $\mathrm{Ca}_{\mathrm{V}} 1.3$ channel population at given active zones. Notably, the exact determinant of the natively observed negative activation of $\mathrm{Ca}_{\mathrm{V}} 1.3$ channels in IHCs at around -60 to $-50 \mathrm{mV}$ (Platzer et al. 2000, Neef et al. 2009) is still unknown. The ratio of Cav1.3 splice-variant expression levels in the organ of Corti seems to be balanced towards the more positively activating full-length channel (Singh et al. 2008), requiring additional mechanisms beyond the reported alternative splicing of $\mathrm{Ca}_{\mathrm{V}} 1.3$. The factors that account for the negatively shifted voltage-dependence of native $\mathrm{Ca}_{\mathrm{V}} 1.3$ currents in IHCs remain at least partly 
elusive. However, their identification may also provide hints towards the mechanisms of differential voltage sensitivity across active different zones.

In conclusion, besides a differential adjustment in terms of $\mathrm{Ca}^{2+}$ channel number, presynaptic $\mathrm{Ca}^{2+}$ influx appears to be variably regulated also at the level of $\mathrm{Ca}_{\mathrm{V}} 1.3$ channel gating. Although the underlying mechanism(s) remain unclear, this potentially provides another stage of modulation of 'synaptic sensitivity' to the receptor potential of IHCs.

From a technical viewpoint, the good match between the average fluorescence-voltage (FV) and the current-voltage (IV) relationship (chapter 2, Figs. 2E, 5A) underlines two important properties of our approach to image synaptic $\mathrm{Ca}^{2+}$ influx: first, even at low open probabilities, correlated with brief mean open times of the hair cell $\mathrm{Ca}^{2+}$ channels (Rodriguez-Contreras and Yamoah 2001), the fluorescence signal mirrors the whole-cell membrane current. That argues against a significant mismatch between the electrophysiological and optical pass bands - although very brief openings are likely to escape optical detection, due to the limited on-rate of the $\mathrm{Ca}^{2+}$ indicator. Second, the large degree of overlap between the two curves (FV and IV) supports the notion of the $\mathrm{Ca}^{2+}$-dependent fluorescence signal being a (local) proxy of the steady-state $\mathrm{Ca}^{2+}$ influx, i.e. the $\mathrm{Ca}^{2+}$ current. The strongest deviation between the two curves is evident at much depolarized potentials, where the whole-cell current reverses its sign (likely due to a $\mathrm{Cs}^{+}$-carried outward current through $\mathrm{Ca}^{2+}$ channels; Hess et al. 1986), while the fluorescence-voltage relationship still reveals $\mathrm{Ca}^{2+}$ influx, even at potentials beyond $+50 \mathrm{mV}$ (chapter 2, Fig. 2E).

\subsection{Other Examples of Synaptic Heterogeneity}

The description of heterogeneity in two key parameters of presynaptic $\mathrm{Ca}^{2+}$ microdomain signals, namely amplitude and voltage-dependence of activation, is expected to have important implications for synaptic function. As mentioned, Griesinger and colleagues reported the observation of differences in de-staining thresholds between different release sites (Griesinger et al. 2005). However, the applicability of the styryl dye FM1-43 for reliable tracking of vesicle cycling in hair cells, including exocytosis, is under debate as several routes of entry into IHCs have been described for the commonly used dyes such as FM1-43. These include (i) endocytosis (Griesinger et al. 2002, Griesinger et al. 2005), (ii) passage through the Mechanotransducer (MET) channel (Gale et al. 2001, Meyers et al. 2003), (iii) and entry via P2X receptors 
(Meyers et al. 2003). While, if contributing significantly in a given experimental preparation, the non-endocytic uptake pathways would severely complicate interpretation of results obtained with FM1-43, it is interesting to note that the larger styryl dye FM3-25 was reported to enter hair cells exclusively via endocytosis (Meyers et al. 2003). However, no use of this dye in the assessment of vesicle cycling in hair cells has been reported to date. It will be interesting to see to which degree the presence of two 18-carbon tails (as compared to two 4-carbon tails in FM1-43) affects the kinetics of membrane-partitioning and de-partitioning and whether this would limit its use as a reporter for fast exocytosis of synaptic vesicles as it occurs in hair cells. Other optical reporters of exocytosis such as pHluorins (Miesenböck et al. 1998) or simultaneous recordings of postsynaptic responses (Glowatzki and Fuchs 2002, Goutman and Glowatzki 2007) are expected to shed more light on the exact relationship between presynaptic $\mathrm{Ca}^{2+}$ microdomain signals, transmitter release, and postsynaptic responses (Singer et al. 2009), Bucurenciu et al. 2010).

\subsection{Tonotopy of $\mathrm{Ca}^{2+}$ Influx in IHCs}

The establishment of optical detection of $\mathrm{Ca}^{2+}$ influx at the level of single active zones in IHCs represents a powerful tool in studying synaptic function. For instance, this technique allowed us to address the physiology of synaptic $\mathrm{Ca}^{2+}$ influx in IHCs at two different tonotopic regions in the adult mouse cochlea (chapter 3, Fig. 5). These experiments were motivated by the finding that whole-cell $\mathrm{Ca}^{2+}$ currents differ in amplitude between IHCs of the apical (low-frequency) and the mid-cochlear (higher frequency) region (chapter 3, Figs. 4d; Supplementary Table 2). The use of non-stationary fluctuation analysis further revealed that these differences are mainly due to a larger number of $\mathrm{Ca}^{2+}$ channels in midcochlear IHCs, although minor differences were also found in unitary current amplitude and maximum open probability (chapter 3, Supplementary Table 2). The marked similarity between all studied aspects of synaptic $\mathrm{Ca}^{2+}$ influx at the two cochlear locations (chapter 3, Fig.5, Table 2), besides the differences in whole-cell $\mathrm{Ca}^{2+}$ influx, lead us to conclude that IHCs use the same 'set' of synapses at different tonotopic positions. This is consistent with the observation that the number of ribbon synapses was increased in midcochlear IHCs (chapter 3, Fig. 1) by $\approx 40 \%$ and that exocytosis could be roughly matched by scaling exocytic responses by synapse number (chapter 3, Fig. 4c). The amplitude variability in the $\mathrm{Ca}^{2+}$ microdomain signals was indistinguishable between both tonotopic regions ( $\mathrm{p}>0.05$ Levene's test (median); $\mathrm{CV}_{\Delta \text { Fpeak,apical }}=0.67$, 
$\mathrm{CV}_{\Delta \text { Fpeak,midcochlear }}=0.57$; chapter 3, Fig. 5b). This observation would be compatible with a potential role of heterogeneous synaptic $\mathrm{Ca}^{2+}$ microdomain signals in sound-coding (see below), as single IHCs appear to be innervated by auditory nerve fibers of different firing characteristics throughout the cochlea (Liberman 1982b). It is furthermore consistent with the observation of indistinguishable Cav1.3 clusters in apical and mid-cochlear IHCs in terms of size and shape (chapter 3; Fig. 3g, Table 1).

An increase in number of synapses and $\mathrm{I}_{\mathrm{Ca}}$ amplitude between low- and higher-frequency regions has also been found for hair cells in the turtle cochlea (Ricci et al. 2000, Schnee et al. 2005). However, unlike in mice, low-frequency hair cells were reported to exhibit on average a significantly larger $\mathrm{Ca}^{2+}$ influx at their synapses (but see: Ricci et al. 2000) - under the assumption that all $\mathrm{Ca}^{2+}$ influx appeared synaptically (but see: Rodriguez-Contreras and Yamoah 2001). This was hypothesized to be related to the limitations imposed by the maximum rate of cycle-to-cycle $\mathrm{Ca}^{2+}$ clearance at higher frequency locations. Indeed, according tonotopic differences in the buffering capacity have been found for these hair cells (Ricci et al. 2000). In mice, where large fractions of the IHC receptor potential are expected to be governed by its dc-component rather than its ac-component (Russell and Sellick 1978, Russell and Sellick 1983), the differences in the ac-/dc-component ratio between two tonotopic positions are likely smaller than in the turtle cochlea. Thus, the magnitude of $\mathrm{Ca}^{2+}$ influx would not need to be adjusted to different requirements of $\mathrm{Ca}^{2+}$ clearance between two frequency locations. Hence, the low characteristic frequencies of the synapses under investigation in the turtle cochlea $(\approx 100 \mathrm{~Hz}$ and $\approx 300 \mathrm{~Hz}$ ) may not be readily comparable to the mechanisms observed in mouse IHCs ( $>1 \mathrm{kHz}$; chapter 3, Fig. 1), especially due to the frequency-differences in receptor potential modulation that governs release in vivo. Nonetheless, the study also indicated that the larger synaptic $\mathrm{Ca}^{2+}$ influx in low-frequency hair cells was correlated with faster release kinetics at these active zones, while the close-to-linear apparent $\mathrm{Ca}^{2+}$-dependence of release, also found in mature IHCs (Brandt et al. 2005, Johnson et al. 2005) was unchanged between the two frequency locations. This provides further evidence for the importance of the magnitude of synaptic $\mathrm{Ca}^{2+}$ influx in determining the rates of neurotransmitter release at active zones.

Consistent with the idea of smaller $\mathrm{Ca}^{2+}$ currents in high-frequency hair cells, the hair cell $\mathrm{Ca}^{2+}$ currents in other high-frequency hearing animals such as birds are generally smaller than in the low-frequency hair cells of frogs or turtles (Martinez-Dunst et al. 1997). As in the 
mouse, the whole-cell amplitude of the current through $\mathrm{Ca}^{2+}$ channels in the chick's basilar papilla (analogous to the mammalian organ of Corti) increased from apex to base, although, opposite to mouse IHCs, it was correlated with larger ribbons and release site area and not ribbon number (Martinez-Dunst et al. 1997). Thus, as in the turtle but unlike in the mouse, a frequency-dependent variation of synaptic $\mathrm{Ca}^{2+}$ influx is suggested for chick hair cells.

Ambiguous results are reported for the tonotopic regulation of $\mathrm{Ca}^{2+}$ influx in adult gerbil IHCs: while one publication reported a slightly smaller size of $\mathrm{I}_{\mathrm{Ca}}$ in apical IHCs (characteristic frequency $\approx 300 \mathrm{~Hz}$; Johnson and Marcotti 2008), a second publication showed the absence of a difference in $\mathrm{I}_{\mathrm{Ca}}$ amplitude between apical and basal IHCs (characteristic frequency $\approx 30 \mathrm{kHz}$; Johnson et al. 2008). The unaltered number of synaptic ribbons per IHC between the two cochlear locations (Johnson et al. 2008) does not point towards differences in synaptic $\mathrm{Ca}^{2+}$ influx as suggested for turtle and chick (see above); yet, this aspect still awaits thorough investigation. In light of the alongside reported observation of tonotopic differences in the apparent $\mathrm{Ca}^{2+}$-dependence of release (Johnson et al. 2008), however, it would be interesting to investigate whether differences in synaptic and/or extrasynaptic $\mathrm{Ca}^{2+}$ influx exist between apical and basal IHCs in the gerbil and whether those could play a role in the interplay between exocytosis and replenishment of synaptic vesicles.

\subsection{Relevance of $\mathrm{Ca}^{2+}$ Microdomain Heterogeneity for Sound Coding}

Now, how exactly could the observed differences in $\mathrm{Ca}^{2+}$ microdomain amplitude of mouse IHCs affect the firing characteristics of postsynaptic ANFs? As laid out in the introduction, there is good evidence for a nanodomain control of transmitter release in IHCs. Under these conditions, exocytosis of glutamate is expected to scale roughly linearly with the magnitude of $\mathrm{Ca}^{2+}$ influx, as an increase in the latter reflects the opening of more $\mathrm{Ca}^{2+}$ channels that reside in close vicinity to synaptic vesicles (Brandt et al. 2005). The large $\left[\mathrm{Ca}^{2+}\right]_{\mathrm{i}}$ excursions close to the channel mouth (Roberts 1994) are expected to saturate the nearby $\mathrm{Ca}^{2+}$ sensor that intrinsically shows a highly non-linear dependence on $\left[\mathrm{Ca}^{2+}\right]_{\mathrm{i}}$ (Beutner et al. 2001). This view is strongly supported by paired recordings from presynaptic hair cells and postsynaptic nerve fibers, both in lower vertebrates (Keen and Hudspeth 2006, Li et al. 2009) and mammals (Goutman and Glowatzki 2007). 
Our reaction-diffusion-imaging simulations moreover suggest that the relationship between the measured Fluo-5N fluorescence and the underlying $\mathrm{Ca}^{2+}$ influx amplitude is nearly linear over the expected range of synaptic $\mathrm{Ca}^{2+}$ influx (chapter 2, Fig. S1). One could therefore expect that synapses with larger $\mathrm{Ca}^{2+}$ microdomain amplitudes release glutamate at higher rates - at least initially, following the onset of a stimulus (see also section 4.6; Schnee et al. 2005, Keen and Hudspeth 2006, Goutman and Glowatzki 2007). However, in order to refine our predictions of $\mathrm{Ca}^{2+}$ microdomain amplitude heterogeneity on auditory nerve fiber 'spiking', we addressed the effect of higher presynaptic release rates in the framework of a published IHC-ANF synapse model (Meddis 1986, Meddis et al. 1990; Fig. A5). Differences in $\mathrm{Ca}^{2+}$ microdomain amplitudes were implemented as scaling of the rate of transmitter release from the presynaptic IHC compartment (Fig. A5A). Varying only this single parameter (' $\mathrm{N}_{\mathrm{Ca}}$ '), the model qualitatively reproduced differences in shape and location of experimentally observed rate-level functions (Fig. A5C, D; Taberner and Liberman 2005, Strenzke et al. 2009). Consistent with these experimental observations, low thresholds in the resulting rate-level functions were correlated with a narrower dynamic range and increased spontaneous rates (Fig. A5C, D) - although the effect of the ' $\mathrm{N}_{\mathrm{Ca}}$ ' scaling on the dynamic range of the model ratelevel functions was modest. Taken together, the general results agree very well with other modeling studies that assigned the presynaptic IHC $\mathrm{Ca}^{2+}$ conductance the major role in the determination of the firing characteristics of auditory nerve fibers (Sumner et al. 2002), Sumner et al. 2003). Moreover, an additional level of specific regulation (and possibly modulation; Wen et al. 2009) of IHC-ANF synaptic transfer functions is provided at the stage of their voltage-dependence (chapter 2, Fig. 5A, section 4.4) - which was not being considered in the above mentioned models. Interestingly, we find larger $\mathrm{Ca}^{2+}$ microdomain signals in the neural sector of IHCs (chapter 3, Fig. 6e) - which was shown to harbor larger ribbons as well as larger and more complex active zones in cats (Merchan-Perez and Liberman 1996). However, ANFs innervating IHCs in these locations were shown to correspond to low-spontaneous rate fibers, while ANFs inserting on the opposite site of the IHCs correspond to highspontaneous rate fibers - at least in cats (Liberman 1982b). Although this observation seems to actually contradict a positive correlation between $\mathrm{Ca}^{2+}$ microdomain amplitude and ANF spontaneous rate (which co-varies with other important ANF firing characteristics; Liberman 1978), it is currently unclear whether this particular spatial segregation of functionally different ANFs exists in mice (Francis et al. 2004). STED estimates of ribbon morphology did not reveal systematic differences between neural and abneural ribbons in mouse IHCs (chapter 3, 
Figs. 6f, g). Furthermore, $\mathrm{Ca}^{2+}$ microdomain heterogeneity persisted in the respective distributions upon separation of $\mathrm{Ca}^{2+}$ microdomain amplitudes in an abneural and a neural population. This raises the possibility that the functional segregation of ANFs in their location of IHC innervation in mice may be less strict as in the cat. Further studies, correlating the structure and function of individual IHC ribbon synapses with the physiology and morphology of their postsynaptic targets will likely provide more insight into the interplay between these parameters in the mouse.

In summary, the observed differences in $\mathrm{Ca}^{2+}$ microdomain properties across synapses present an interesting candidate mechanism for the heterogeneous sensitivities observed in auditory nerve fibers. While $\mathrm{Ca}^{2+}$ channel number could play an important role by itself, differential adjustment of the voltage-sensitivity of the presynaptic $\mathrm{Ca}^{2+}$ conductance may provide a second level of modulation.

However, our findings by no way rule out any contribution of efferent top-down modulation (e.g. Ruel et al. 2001) and/or postsynaptic (e.g. Mo and Davis 1997) and/or other presynaptic mechanisms (e.g. Singer et al. 2009) on the determination of ANF firing. Future studies, combining presynaptic $\mathrm{Ca}^{2+}$ microdomain measurements with simultaneous readout of exocytosis or postsynaptic responses at the single-synaptic level will likely further deepen our understanding of the mechanisms determining the information transfer at the first auditory synapse (Goutman and Glowatzki 2007) and especially its regulation on the single-synapse level. 


\section{Summary}

Using whole-cell patch-clamp and fast confocal $\mathrm{Ca}^{2+}$ imaging we show that localized $\mathrm{Ca}^{2+}$ influx sites exist at ribbon synapses in mature inner hair cells of the mouse. Spot detection measurements at these fluorescent hot-spots allowed their precise kinetic characterization, revealing rapid build-up and cessation within few milliseconds. We find that these $\mathrm{Ca}^{2+}$ microdomains represent $\mathrm{Ca}^{2+}$ influx through presynaptic clusters of $\mathrm{Ca}_{\mathrm{v}} 1.3$ channels and that $\mathrm{Ca}^{2+}$-induced $\mathrm{Ca}^{2+}$ release does not play a role in shaping these responses. They were observed under a range of different $\mathrm{Ca}^{2+}$ buffering conditions - including the presence of endogenous buffers. A combined model of $\mathrm{Ca}^{2+}$ reaction-diffusion and imaging predicted the experimentally observed responses reasonably well, under a set of different conditions. The amplitude and the voltage-dependence of these $\mathrm{Ca}^{2+}$ microdomains showed pronounced variability across individual synapses within single cells. A number of control experiments indicate that this variability is largely of biological origin and only to a minor degree introduced by the experimental observations. Different manipulations, including interference with $\mathrm{Ca}^{2+}$ buffering, $\mathrm{Ca}^{2+}$ channel gating and $\mathrm{Ca}^{2+}$ homeostasis left the variability of $\mathrm{Ca}^{2+}$ microdomain amplitudes unchanged. However, we found substantial variability in the antigen-content between presynaptic $\mathrm{Ca}^{2+}$ channel clusters as well as a positive correlation between the size of the active zone - as indicated by a size estimate of the synaptic ribbon - and the amplitude of its associated $\mathrm{Ca}^{2+}$ microdomain signal. We propose that individual active zones within a given hair cell differ in the number and gating of $\mathrm{Ca}^{2+}$ channels, resulting in different amplitudes and voltage-dependencies of synaptic $\mathrm{Ca}^{2+}$ influx. These specializations provide two levels of regulation to differentially adjust synaptic transfer characteristics at individual synapses. Implementation of our results into a model of the inner hair cell to auditory nerve fiber synapse further suggests a strong impact of presynaptic $\mathrm{Ca}^{2+}$ channel number on the firing characteristics of the postsynaptic auditory nerve fibers and thus sound coding. We apply this technique to investigate presynaptic function at different tonotopic locations, revealing that - unlike in other species - mice do not adjust the characteristics but rather the number of IHC ribbon synapses in a frequency-dependent manner. 


\section{Appendix}

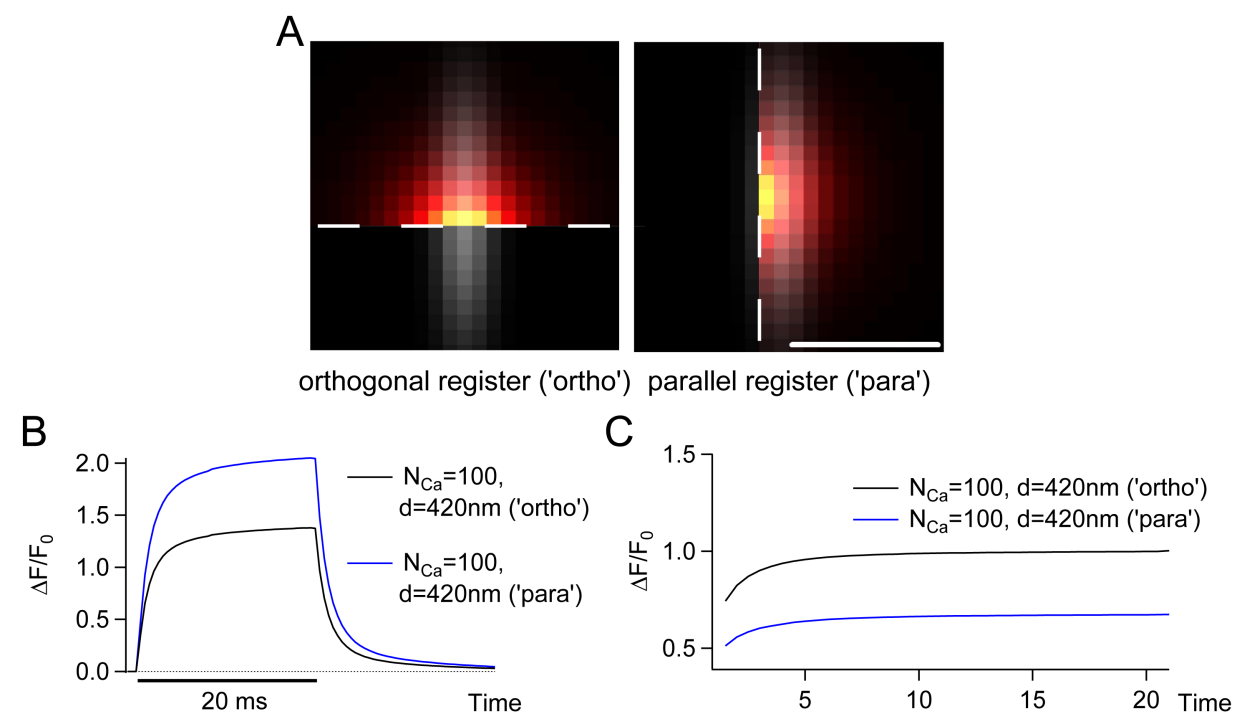

Figure A 1 | Simulated effect of spatial relationship between excitation-detection volume and $\mathrm{Ca}^{2+}$ influx site on amplitude and width of measured $\mathrm{Ca}^{2+}$ microdomain. (A) Schematic illustration of the two considered orientations between the simulated $\left[\mathrm{Ca}^{2+}\right.$-indicator] profile (black-redyellow color scale, yellow indicating largest concentration) and the simplified excitation detection volume (point spread function, PSF; gray-scale overlay, white indicating largest intensity). The white broken line indicates the location of the reflective boundary (plasma membrane); scale bar represents $1 \mu \mathrm{m}$. Both images show a cutout of the center plane of the simulation volume (cubic, with a total volume of $(5.1 \mu \mathrm{m})^{3}$; see also: chapter 2 , Table S3). The optical axis $(Z)$ runs vertically and is indicated by the long dimension of the PSF. In contrast to the simulations presented in chapter 2, we simulated a slightly larger and square-shaped $\mathrm{Ca}^{2+}$ influx site (420 nm (diameter) vs. $300 \mathrm{~nm}$ (FWHM)), to meet experimental data (chapter 3). All other parameters as in Table S3 (chapter 2). (B) Resulting fluorescence traces for both configurations, reflecting the different orientation between $\mathrm{Ca}^{2+}$ influx site and PSF. $\mathrm{Ca}^{2+}$ influx was simulated for $20 \mathrm{~ms}$, starting after $1 \mathrm{~ms}$ resting period. Both traces represent the respective maximum intensity traces. The maximum amplitude of the orthogonal configuration ('ortho') amounted to $67 \%$ of the parallel registration between $\mathrm{Ca}^{2+}$ influx site and PSF ('para'). $\mathrm{N}_{\mathrm{Ca}}$ denotes magnitude of $\mathrm{Ca}^{2+}$ influx (number of $\mathrm{Ca}^{2+}$ channels), $\mathrm{d}$ indicates the diameter of the $\mathrm{Ca}^{2+}$ influx site. (C) Corresponding line-scan profiles over time for the two configurations. Full width at halfmaximum (FWHM) was estimated by fitting Gaussian functions to simulated line-scans for both configurations (see also: chapter 2, Fig. S1b). The FWHM of the simulated $\mathrm{Ca}^{2+}$ microdomain at the end of the stimulation in the orthogonal configuration was $49 \%$ larger than in the parallel configuration. 

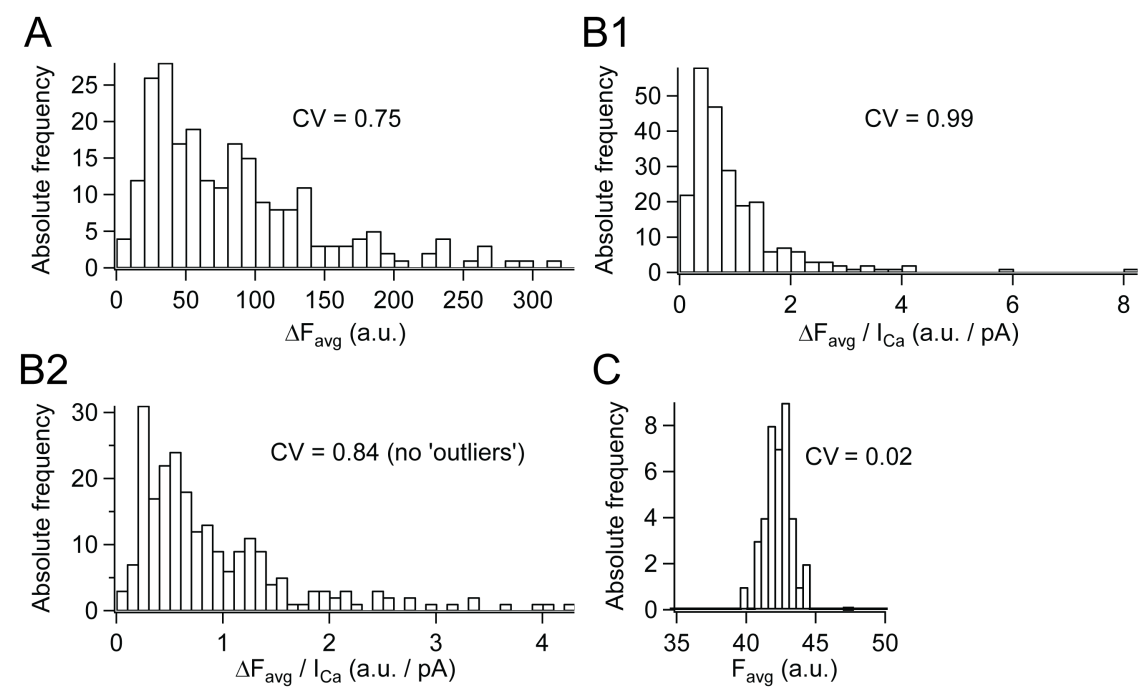

Figure A 2 | Distributions of $\mathrm{Ca}^{2+}$ microdomain amplitudes $(\mathrm{A}, \mathrm{B})$ and fluorescein control spot detection measurements (C). (A) Distribution of $\Delta \mathrm{F}_{\text {avg }}$ (average fluorescence change, $\Delta \mathrm{F}$, calculated over the last $10 \mathrm{~ms}$ ) for $231 \mathrm{Ca}^{2+}$ microdomains from 73 IHCs (P14-P31). CV abbreviates coefficient of variation. In contrast to the measurements shown in chapters 2 and 3, these experiments were performed using a different lot of Fluo-5N (Lot-No.: 488270 (these experiments); Lot-No.: 28814W (chapter 2, 3); both lots were purchased from Invitrogen). In vitro- and in situ calibration confirmed a $\approx 2$.6-fold larger $\mathrm{F}_{\max } / \mathrm{F}_{\min }$ ratio for the lot 488270 . (B1) Distribution of $\mathrm{Ca}^{2+}$ microdomain amplitudes normalized to simultaneously acquired $\mathrm{Ca}^{2+}$ influx. Based on the distribution shown in (A), however $\Delta \mathrm{F}_{\text {avg }}$ values were divided by the mean whole-cell $\mathrm{I}_{\mathrm{Ca}}$ amplitude during the last $10 \mathrm{~ms}$ of a $20 \mathrm{~ms}$ stimulus to $-7 \mathrm{mV})$. Recording conditions were as described in chapter $2\left(\mathrm{I}_{\mathrm{Ca}}\right.$ recorded in the presence of intra- and extracellular $\mathrm{Cs}^{+}$and tetraethylammonium ions, and was further isolated using $\mathrm{p} / \mathrm{n}$ correction). Note that the sign of the current amplitude was inverted for calculation, yielding positive values of $\triangle \mathrm{F} / \mathrm{pA}$. (B2) The very same distribution as shown in $\mathrm{B} 1$ but after removal of two outliers (seen in $\mathrm{B} 1$ at $\approx 6$ a.u./pA and $\approx 8$ a.u./pA). However, it is evident from both distributions that the normalization of the $\mathrm{Ca}^{2+}$ microdomain amplitude to the whole $\mathrm{Ca}^{2+}$ influx (potentially correcting for different physiological conditions of IHCs) did not lead to a reduction in the observed amplitude heterogeneity - as judged by the CVs of the distributions. (C) Summary of control spot detection experiments performed in a bath of $100 \mu \mathrm{M}$ freshly prepared fluorescein. Distribution shows mean (raw) fluorescence amplitudes (averaged over $10 \mathrm{~ms}$ and preceded by $\approx 60 \mathrm{~ms}$ illumination; imaging was performed at reduced laser intensity to match the fluorescence amplitudes to the amplitudes of the experimentally observed $\mathrm{Ca}^{2+}$ microdomain signals). Single spot detection recordings were performed as on $\mathrm{Ca}^{2+}$ influx sites, i.e. the analyzed traces represented averages of 5 repetitions at the same location. Note the small CV that provides an estimate for the lower boundary of the variance contribution by the imaging setup in the reported spot detection experiments. 

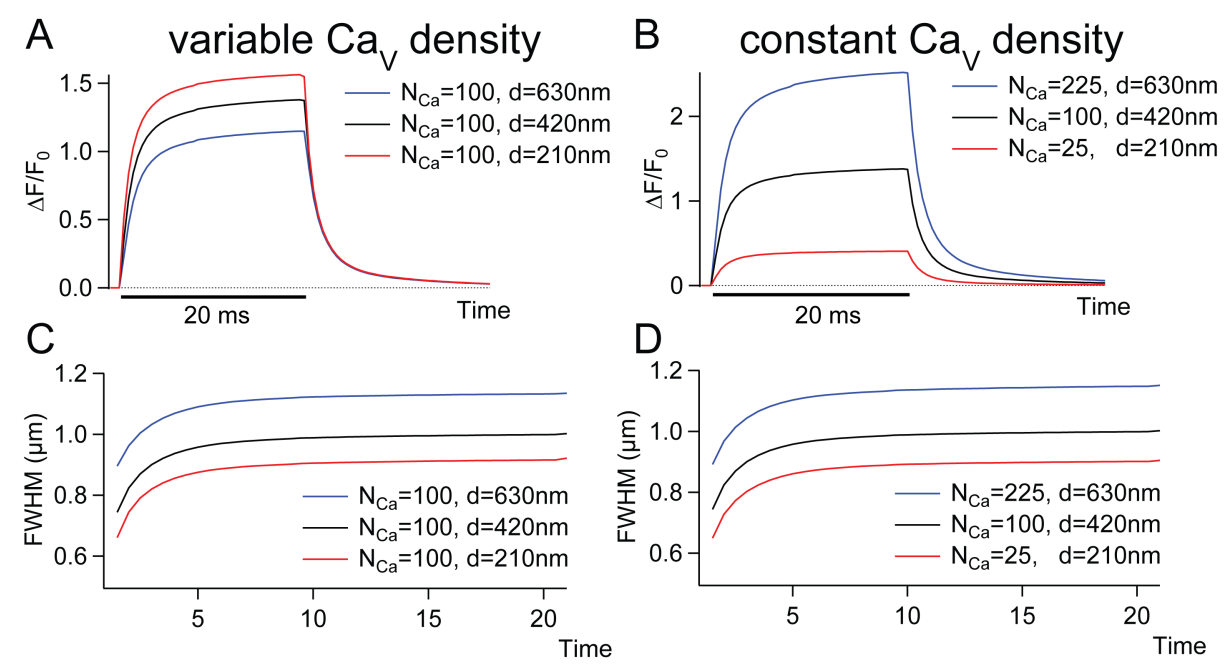

Figure A 3 | Simulated spot detection measurements at variable $(\mathrm{A}, \mathrm{C})$ or constant $\mathrm{Ca}^{2+}$ influx density (B, D) - with varying size of $\mathrm{Ca}^{2+}$ influx site. All simulation parameters and abbreviations as introduced in Fig. A1; detection simulated under orthogonal registration of PSF and $\mathrm{Ca}^{2+}$ influx site. (A) Small effect of size-differences of $\mathrm{Ca}^{2+}$ influx site at unchanged amplitude of $\mathrm{Ca}^{2+}$ influx (variable $\mathrm{Ca}^{2+}$ influx density) on predicted spot detection amplitude at the center of the $\mathrm{Ca}^{2+}$ influx site. The maximum amplitude (at the end of the $20 \mathrm{~ms} \mathrm{Ca}^{2+}$ influx period) for the smallest influx site $(\mathrm{d}=210 \mathrm{~nm})$ was $36 \%$ larger than the amplitude for the largest $\mathrm{Ca}^{2+}$ influx site $(\mathrm{d}=630 \mathrm{~nm})$. (B) At a constant density of $\mathrm{Ca}^{2+}$ influx, the smallest $\mathrm{Ca}^{2+}$ influx site ('210 nm') showed an $84 \%$ smaller amplitude than the largest $\mathrm{Ca}^{2+}$ influx site ('630 nm'). (C) Time-dependent FWHM of the corresponding simulated line-scans across $\mathrm{Ca}^{2+}$ influx sites with variable $\mathrm{Ca}^{2+}$ influx density. For the smallest $\mathrm{Ca}^{2+}$ influx site ('210 nm'), a $20 \%$ narrower $\mathrm{Ca}^{2+}$ microdomain was predicted by the model. (D) As in (C), but at constant $\mathrm{Ca}^{2+}$ influx density (see (B)). The predicted size of the $\mathrm{Ca}^{2+}$ microdomain was unaffected by the $\mathrm{Ca}^{2+}$ influx density $\left(\mathrm{d}_{210 \mathrm{~nm}}=0.79 \mathrm{x} \mathrm{d}_{420 \mathrm{~nm}}\right)$. Note the same scaling of the abscissa.

These simulations indicate that the imaging system is generally more sensitive to the absolute amount of $\mathrm{Ca}^{2+}$ influx rather than its density (A, B), although the amplitude will be underestimated at large $\mathrm{Ca}^{2+}$ influx sites due to the smaller PSF (Fig. 1A). Furthermore, the line-scan-based estimation of $\mathrm{Ca}^{2+}$ microdomain width is predicted not to be affected by the amplitude of $\mathrm{Ca}^{2+}$ influx. However, the uncertainty about the orientation of the PSF with regard to the $\mathrm{Ca}^{2+}$-influx site within the actual experiments and the resulting ambiguity about the underlying size of the $\mathrm{Ca}^{2+}$ influx site (see also Fig. 1) precludes even relative density estimations, unless the exact orientation of the respective $\mathrm{Ca}^{2+}$ influx site to the optical axis was known. 
A

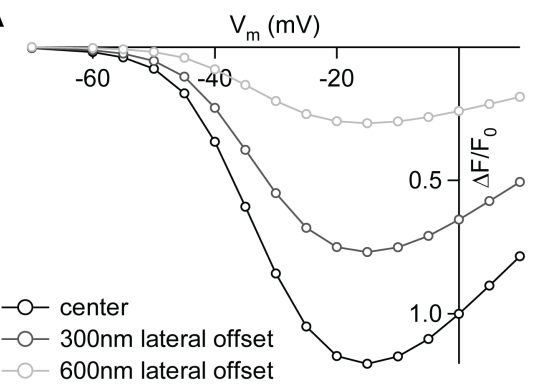

B

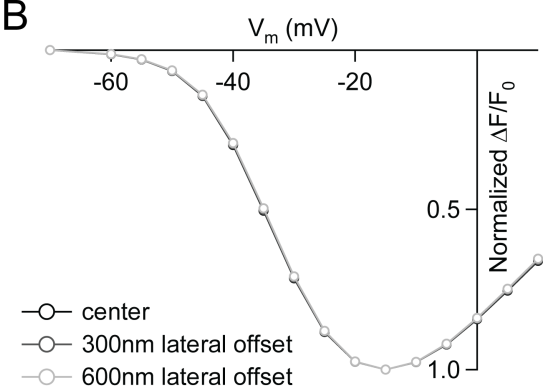

Figure A 4 | Simulated effect of the PSF-Ca ${ }^{2+}$ influx site register on the voltage-dependence of the observed $\mathrm{Ca}^{2+}$ microdomain. (A) Fluorescence-voltage curves were assembled using independent simulations $\mathrm{Ca}^{2+}$ influx at a $420 \mathrm{~nm}$ site. The $\mathrm{Ca}^{2+}$ indicator in these simulations was Fluo-5N ([Fluo-5N $]_{\text {total }}=400 \mu \mathrm{M}$ ), [EGTA $]_{\text {total }}$ was set to $2 \mathrm{mM}$ (see also: chapter 2, Table S3). The unitary $\mathrm{Ca}^{2+}$ current at each potential $\left(\mathrm{V}_{\mathrm{m}}\right)$ was estimated from published values for L-type $\mathrm{Ca}^{2+}$ channels in chick ciliary ganglion cells (in $4 \mathrm{mM}\left[\mathrm{Ca}^{2+}\right]_{\mathrm{e}}$; Church and Stanley 1996). The respective voltagedependence of open probability was derived from an average conductance-voltage relationship (chapter 2; Fig. 5E), and the maximal open probability at maximal conductance set to 0.4 (Brandt et al. 2005). All other simulation parameters were as described (chapter 2, Table S3; Fig. 1A). To arrive at the three different curves shown in (A), the simulated PSF (orthogonal oriented; see Fig. 1A) was shifted laterally by the indicated distance $(0 \mathrm{~nm}, 300 \mathrm{~nm}, 600 \mathrm{~nm})$ from the center of the $\mathrm{Ca}^{2+}$ influx site. This resulted in an expected decrease in predicted amplitude, as less $\mathrm{Ca}^{2+}$ influx is actually detected by the simulated PSF. (B) Very strong overlap in the peak-normalized curves from (A). This result suggests that the observed heterogeneity in the voltage-dependence of activation of $\mathrm{Ca}^{2+}$ microdomains (chapter 2, Fig. 5A) cannot be explained by artifactual mis-localization of the PSF with regard to the $\mathrm{Ca}^{2+}$ influx site. 
A

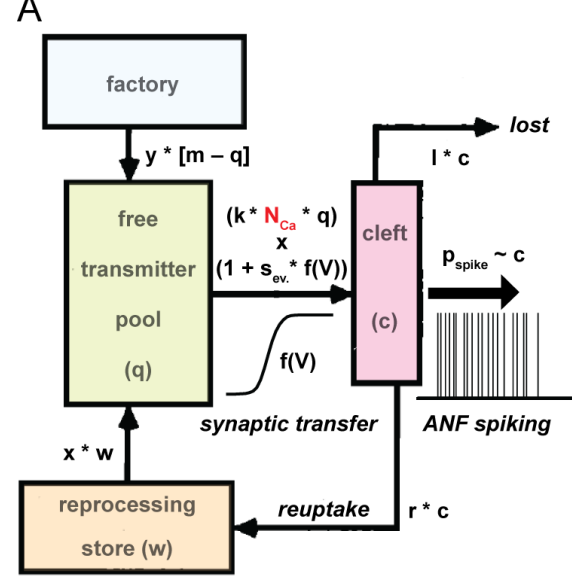

B

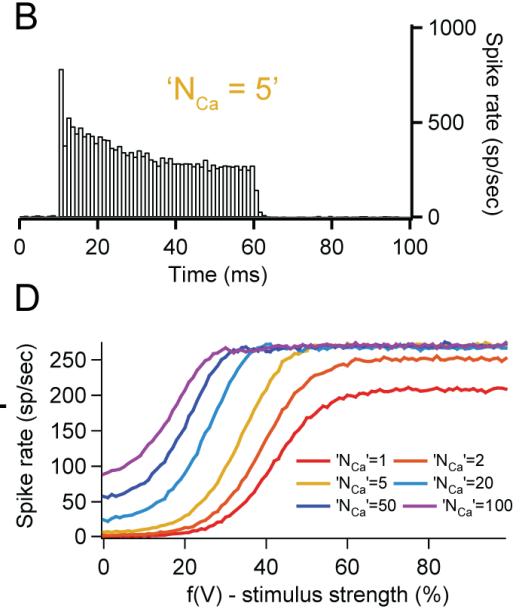

C
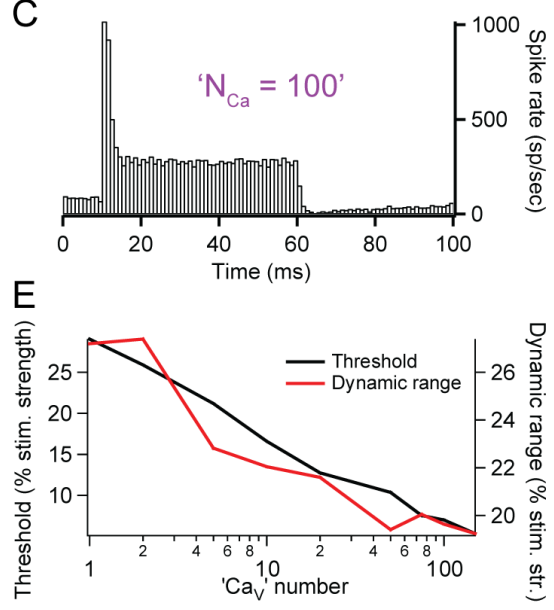

Figure A 5 | Effect of presynaptic release-rate scaling on simulated ANF firing behaviour in a model of the IHC-ANF synapse. (A) Scheme of IHC-ANF synapse model (modified from (Meddis 1990)), using a set of ordinary differential equations. In the center of this model is the transmitter content of the synaptic cleft (c). The probability of spike initiation ( $\mathrm{p}_{\text {spike; }}$ ' ANF spiking') in the ANF is set to depend on the cleft content (c). The cleft transmitter content (c) is increased by release from the free transmitter pool within the hair cell (q; 'synaptic transfer'), and decreased both by loss from the synaptic cleft (loss rate $\left.1^{*} \mathrm{c}(\mathrm{t}) \mathrm{s}^{-1}\right)$ and reuptake (reuptake rate $\mathrm{r}^{*} \mathrm{c}(\mathrm{t}) \mathrm{s}^{-1}$ ) into a reprocessing store $(\mathrm{w})$. This reprocessing store, in turn, increases the free transmitter content (q), at a rate $\mathrm{x}^{*} \mathrm{w}(\mathrm{t}) \mathrm{s}^{-1}-$ in conjunction with de novo synthesis (at a rate $\mathrm{y}^{*}\left(\mathrm{~m}-\mathrm{q}(\mathrm{t}) \mathrm{s}^{-1}\right)$ of transmitter from a 'factory' (with $\mathrm{m}$ being the maximum amount of transmitter in the system). Within the 'synaptic transfer' section, the release rate is simulated as a baseline rate $\left(\mathrm{k}\right.$ ), multiplied by a factor ' $\mathrm{N}_{\mathrm{Ca}}$ ' (termed according to $\mathrm{Ca}^{2+}$ channel number) times the free transmitter pool content q. With increasing stimulation, release is further scaled according to a sigmoidal function, following the voltage-dependence of the $\mathrm{IHC} \mathrm{Ca}^{2+}$ conductance (modeled based on average conductance-voltage relationship). The maximal scaling factor of this evoked rate by stimulation was set to 500 , on the basis of measured maximal increases of vesicle fusion rates in IHCs at saturating $\left[\mathrm{Ca}^{2+}\right]_{\mathrm{i}}$ (Beutner et al. 2001). The probabilistic spike generation was modeled with an exponential distribution that was scaled by the cleft transmitter content (c) and modulated by absolute $(0.8 \mathrm{~ms})$ and relative refractoriness. Relative refractoriness recovered exponentially with a time constant of $2 \mathrm{~ms}$. The model was implemented in Igor Pro 6 (Wavemetrics). (B) Example of a simulated post-stimulus time histogram (PSTH) at saturating 'stimulus strength' (i.e. at the plateau of the sigmoidal function) from a synapse with a scaling factor $\left(\mathrm{N}_{\mathrm{Ca}}\right)$ of 5 . The 'stimulus' was active for $50 \mathrm{~ms}$, starting at the time point of $10 \mathrm{~ms}$. This PSTH represents simulated data from 100 runs. It qualitatively reproduces characteristic features of, such as slow adaptation and sparse firing in the absence of a 'stimulus' (e.g. Liberman 1978, Taberner and Liberman 2005). (C) As in (B) but with a scaling factor (' $\mathrm{N}_{\mathrm{Ca}}$ ') of 100 . Note the pronounced activity in the absence of 'stimulation', the fast adaptation of the response, and the slow recovery of the spontaneous spiking following the 'stimulus' period, typical features of high-spontaneous rate auditory nerve fibers (see ref. above). (D) Summary plot, showing rate-level functions of different simulated synapses that only varied in their scaling factor (' $\mathrm{N}_{\mathrm{Ca}}$ ') as indicated. (E) Summary plot, showing the correlation between the scaling factor (' $\mathrm{N}_{\mathrm{Ca}}$ ') and threshold (defined as 10\% increase above the firing rate in the absence of a 'stimulus') as well as dynamic range (defined as 10-90\% range of firing rate increase) of the simulated auditory nerve fibers. Qualitatively, these observations agree with experimental observations (see ref. above). 


\section{Bibliography}

Adamson CL, Reid MA, Mo Z, Bowne-English J, Davis RL. 2002. Firing features and potassium channel content of murine spiral ganglion neurons vary with cochlear location. $J$ Comp Neurol 447: 331-50.

Arnold W. 1974. Adrenerge Synapsen im Bereich der Habenula perforata. Arch Otorhinolaryngol 208: 277-282.

Ashmore J. 2008. Cochlear outer hair cell motility. Physiol Rev 88: 173-210.

Ashmore J, Avan P, Brownell WE, Dallos P, Dierkes K, Fettiplace R, Grosh K, Hackney C, Hudspeth AJ, Jülicher F, Lindner B, Martin P, Meaud J, Petit C, Santos Sacchi JR, Canlon B. 2010. The remarkable cochlear amplifier. Hear Res.

Augustine GJ, Santamaria F, Tanaka K. 2003. Local calcium signaling in neurons. Neuron 40: $331-46$.

Bakondi G, Pór A, Kovács I, Szucs G, Rusznák Z. 2008. Voltage-gated K+ channel (Kv) subunit expression of the guinea pig spiral ganglion cells studied in a newly developed cochlear free-floating preparation. Brain Res 1210: 148-62.

Baroudi G, Qu Y, Ramadan O, Chahine M, Boutjdir M. 2006. Protein kinase C activation inhibits Cav1.3 calcium channel at NH2-terminal serine 81 phosphorylation site. Am J Physiol Heart Circ Physiol 291: H1614-22.

Beurg M, Fettiplace R, Nam J, Ricci AJ. 2009. Localization of inner hair cell mechanotransducer channels using high-speed calcium imaging. Nat Neurosci 12: 553-8.

Beutner D, Moser T. 2001. The presynaptic function of mouse cochlear inner hair cells during development of hearing. J Neurosci 21: 4593-9.

Beutner D, Voets T, Neher E, Moser T. 2001. Calcium dependence of exocytosis and endocytosis at the cochlear inner hair cell afferent synapse. Neuron 29: 681-90.

Borst JG, Sakmann B. 1996. Calcium influx and transmitter release in a fast CNS synapse. Nature 383: 431-4.

Brandt A, Khimich D, Moser T. 2005. Few CaV1.3 channels regulate the exocytosis of a synaptic vesicle at the hair cell ribbon synapse. J Neurosci 25: 11577-85.

Brandt A, Striessnig J, Moser T. 2003. CaV1.3 channels are essential for development and presynaptic activity of cochlear inner hair cells. J Neurosci 23: 10832-40.

Brugge JF, Howard MA. 2002. Hearing. Encyclopedia of the Human Brain 2: 429-448.

Bucurenciu I, Bischofberger J, Jonas P. 2010. A small number of open Ca2+ channels trigger transmitter release at a central GABAergic synapse. Nat Neurosci 13: 19-21. 
Catterall WA. 2000. Structure and regulation of voltage-gated Ca2+ channels. Annu Rev Cell Dev Biol 16: 521-55.

Church PJ, Stanley EF. 1996. Single L-type calcium channel conductance with physiological levels of calcium in chick ciliary ganglion neurons. J Physiol 496 ( Pt 1: 59-68.

Colburn HS, Carney LH, Heinz MG. 2003. Quantifying the information in auditory-nerve responses for level discrimination. J Assoc Res Otolaryngol 4: 294-311.

Cui G, Meyer AC, Calin-Jageman I, Neef J, Haeseleer F, Moser T, Lee A. 2007. Ca2+binding proteins tune $\mathrm{Ca} 2+$-feedback to Cav1.3 channels in mouse auditory hair cells. $J$ Physiol 585: 791-803.

Dai S, Hall DD, Hell JW. 2009. Supramolecular assemblies and localized regulation of voltage-gated ion channels. Physiol Rev 89: 411-52.

Dallos P. 1985. Response characteristics of mammalian cochlear hair cells. J Neurosci 5: 1591-608.

Dargan SL, Parker I. 2003. Buffer kinetics shape the spatiotemporal patterns of IP3-evoked Ca2+ signals. $J$ Physiol 553: 775-88.

Darrow KN, Maison SF, Liberman MC. 2006. Cochlear efferent feedback balances interaural sensitivity. Nat Neurosci 9: 1474-6.

Darrow KN, Maison SF, Liberman MC. 2007. Selective removal of lateral olivocochlear efferents increases vulnerability to acute acoustic injury. $J$ Neurophysiol 97: 1775-85.

Dean I, Harper NS, McAlpine D. 2005. Neural population coding of sound level adapts to stimulus statistics. Nat Neurosci 8: 1684-9.

Edmonds B, Reyes R, Schwaller B, Roberts WM. 2000. Calretinin modifies presynaptic calcium signaling in frog saccular hair cells. Nat Neurosci 3: 786-90.

Ehret G. 1985. Behavioural studies on auditory development in mammals in relation to higher nervous system functioning. Acta Otolaryngol Suppl 421: 31-40.

Elgoyhen AB, Vetter DE, Katz E, Rothlin CV, Heinemann SF, Boulter J. 2001. alpha10: a determinant of nicotinic cholinergic receptor function in mammalian vestibular and cochlear mechanosensory hair cells. Proc Natl Acad Sci U S A 98: 3501-6.

Eybalin M. 1993. Neurotransmitters and neuromodulators of the mammalian cochlea. Physiol Rev 73: 309-73.

Faas GC, Schwaller B, Mody I, Vergara JL. 2007. Resolving the fast kinetics of cooperative binding: Ca2+ buffering by calretinin. PLoS biology 5: e311.

Fettiplace R. 2009. Defining features of the hair cell mechanoelectrical transducer channel. Pflugers Arch 458: 1115-23. 
Fettiplace R, Fuchs PA. 1999. Mechanisms of hair cell tuning. Annu Rev Physiol 61: 809-34.

Fettiplace R, Hackney CM. 2006. The sensory and motor roles of auditory hair cells. Nat Rev Neurosci 7: 19-29.

Francis HW, Rivas A, Lehar M, Ryugo DK. 2004. Two types of afferent terminals innervate cochlear inner hair cells in C57BL/6J mice. Brain Res 1016: 182-94.

Gale JE, Marcotti W, Kennedy HJ, Kros CJ, Richardson GP. 2001. FM1-43 dye behaves as a permeant blocker of the hair-cell mechanotransducer channel. J Neurosci 21: 7013-25.

Glowatzki E, Fuchs PA. 2000. Cholinergic synaptic inhibition of inner hair cells in the neonatal mammalian cochlea. Science 288: 2366-8.

Glowatzki E, Fuchs PA. 2002. Transmitter release at the hair cell ribbon synapse. Nat Neurosci 5: 147-54.

Glowatzki E, Cheng N, Hiel H, Yi E, Tanaka K, Ellis-Davies GC, Rothstein JD, Bergles DE. 2006. The glutamate-aspartate transporter GLAST mediates glutamate uptake at inner hair cell afferent synapses in the mammalian cochlea. $J$ Neurosci 26: 7659-64.

Glowatzki E, Grant L, Fuchs P. 2008. Hair cell afferent synapses. Curr Opin Neurobiol 18: 389-95.

Goutman JD, Glowatzki E. 2007. Time course and calcium dependence of transmitter release at a single ribbon synapse. Proc Natl Acad Sci U S A 104: 16341-6.

Grant L, Slapnick S, Kennedy H, Hackney C. 2006. Ryanodine receptor localisation in the mammalian cochlea: an ultrastructural study. Hear Res 219: 101-9.

Griesinger CB, Richards CD, Ashmore JF. 2002. Fm1-43 reveals membrane recycling in adult inner hair cells of the mammalian cochlea. J Neurosci 22: 3939-3952.

Griesinger CB, Richards CD, Ashmore JF. 2005. Fast vesicle replenishment allows indefatigable signalling at the first auditory synapse. Nature 435: 212-5.

Groff JA, Liberman MC. 2003. Modulation of cochlear afferent response by the lateral olivocochlear system: activation via electrical stimulation of the inferior colliculus. J Neurophysiol 90: 3178-200.

Hackney CM, Mahendrasingam S, Jones EM, Fettiplace R. 2003. The distribution of calcium buffering proteins in the turtle cochlea. J Neurosci 23: 4577-89.

Hackney CM, Mahendrasingam S, Penn A, Fettiplace R. 2005. The concentrations of calcium buffering proteins in mammalian cochlear hair cells. $J$ Neurosci 25: 7867-75.

He L, Xue L, Xu J, McNeil BD, Bai L, Melicoff E, Adachi R, Wu L. 2009. Compound vesicle fusion increases quantal size and potentiates synaptic transmission. Nature 459: 93-7. 
Hess P, Lansman JB, Tsien RW. 1984. Different modes of Ca channel gating behaviour favoured by dihydropyridine $\mathrm{Ca}$ agonists and antagonists. Nature 311: 538-44.

Hess P, Lansman JB, Tsien RW. 1986. Calcium channel selectivity for divalent and monovalent cations. Voltage and concentration dependence of single channel current in ventricular heart cells. J Gen Physiol 88: 293-319.

Housley GD, Marcotti W, Navaratnam D, Yamoah EN. 2006. Hair cells--beyond the transducer. J Membr Biol 209: 89-118.

Hudspeth AJ. 2008. Making an effort to listen: mechanical amplification in the ear. Neuron 59: $530-45$.

Issa NP, Hudspeth AJ. 1994. Clustering of $\mathrm{Ca} 2+$ channels and $\mathrm{Ca}(2+)$-activated $\mathrm{K}+$ channels at fluorescently labeled presynaptic active zones of hair cells. Proc Natl Acad Sci US A 91: 7578-82.

Issa NP, Hudspeth AJ. 1996. The entry and clearance of $\mathrm{Ca} 2+$ at individual presynaptic active zones of hair cells from the bullfrog's sacculus. Proc Natl Acad Sci U S A 93: 9527-32.

Johnson SL, Marcotti W. 2008. Biophysical properties of CaV1.3 calcium channels in gerbil inner hair cells. J Physiol 586: 1029-42.

Johnson SL, Forge A, Knipper M, Münkner S, Marcotti W. 2008. Tonotopic variation in the calcium dependence of neurotransmitter release and vesicle pool replenishment at mammalian auditory ribbon synapses. $J$ Neurosci 28: 7670-8.

Johnson SL, Franz C, Kuhn S, Furness DN, Rüttiger L, Münkner S, Rivolta MN, Seward EP, Herschman HR, Engel J, Knipper M, Marcotti W. 2010. Synaptotagmin IV determines the linear $\mathrm{Ca} 2+$ dependence of vesicle fusion at auditory ribbon synapses. Nat Neurosci 13: 45-52.

Johnson SL, Marcotti W, Kros CJ. 2005. Increase in efficiency and reduction in Ca2+ dependence of exocytosis during development of mouse inner hair cells. $J$ Physiol 563: 177-91.

Kazmierczak P, Sakaguchi H, Tokita J, Wilson-Kubalek EM, Milligan RA, Müller U, Kachar B. 2007. Cadherin 23 and protocadherin 15 interact to form tip-link filaments in sensory hair cells. Nature 449: 87-91.

Keen EC, Hudspeth AJ. 2006. Transfer characteristics of the hair cell's afferent synapse. Proc Natl Acad Sci U S A 103: 5537-42.

Kennedy HJ. 2002. Intracellular calcium regulation in inner hair cells from neonatal mice. Cell calcium 31: 127-36.

Kennedy HJ, Meech RW. 2002. Fast $\mathrm{Ca} 2+$ signals at mouse inner hair cell synapse: a role for $\mathrm{Ca} 2+$-induced $\mathrm{Ca} 2+$ release. J Physiol 539: 15-23. 
Khimich D, Nouvian R, Pujol R, Tom Dieck S, Egner A, Gundelfinger ED, Moser T. 2005. Hair cell synaptic ribbons are essential for synchronous auditory signalling. Nature 434: 889-94.

Kiang NY, Watanabe T, Thomas EC, Clarke LF. 1965. Discharge Patterns of Single Fibers in the Cats Auditory Nerve. Cambridge, MA: MIT Press.

Kozlov AS, Risler T, Hudspeth AJ. 2007. Coherent motion of stereocilia assures the concerted gating of hair-cell transduction channels. Nat Neurosci 10: 87-92.

Kros CJ, Crawford AC. 1990. Potassium currents in inner hair cells isolated from the guinea-pig cochlea. J Physiol 421: 263-91.

Kros CJ, Ruppersberg JP, Rüsch A. 1998. Expression of a potassium current in inner hair cells during development of hearing in mice. Nature 394: 281-4.

Kumagami H, Beitz E, Wild K, Zenner H-, Ruppersberg JP, Schultz JE. 1999. Expression pattern of adenylyl cyclase isoforms in the inner ear of the rat by RT-PCR and immunochemical localization of calcineurin in the organ of Corti. Hear Res 132: 69-75.

Legan PK, Lukashkina VA, Goodyear RJ, Kössi M, Russell IJ, Richardson GP. 2000. A targeted deletion in alpha-tectorin reveals that the tectorial membrane is required for the gain and timing of cochlear feedback. Neuron 28: 273-85.

Lelli A, Perin P, Martini M, Ciubotaru CD, Prigioni I, Valli P, Rossi ML, Mammano F. 2003. Presynaptic calcium stores modulate afferent release in vestibular hair cells. J Neurosci 23: 6894-903.

Lenzi D, von Gersdorff H. 2001. Structure suggests function: the case for synaptic ribbons as exocytotic nanomachines. Bioessays 23: 831-40.

Lenzi D, Runyeon JW, Crum J, Ellisman MH, Roberts WM. 1999. Synaptic vesicle populations in saccular hair cells reconstructed by electron tomography. J Neurosci 19: 119-32.

Li G, Keen E, Andor-Ardó D, Hudspeth AJ, von Gersdorff H. 2009. The unitary event underlying multiquantal EPSCs at a hair cell's ribbon synapse. J Neurosci 29: 7558-68.

Liang Y, Tavalin SJ. 2007. Auxiliary beta subunits differentially determine pka utilization of distinct regulatory sites on Cav1.3 L type Ca2+ channels. Channels 1: 102-12.

Liberman MC. 1978. Auditory-nerve response from cats raised in a low-noise chamber. $J$ Acoust Soc Am 63: 442-55.

Liberman MC. 1980. Efferent synapses in the inner hair cell area of the cat cochlea: an electron microscopic study of serial sections. Hear Res 3: 189-204.

Liberman MC. 1982. Single-neuron labeling in the cat auditory nerve. Science 216: 12391241. 
Liberman MC. 1982. The cochlear frequency map for the cat: labeling auditory-nerve fibers of known characteristic frequency. $J$ Acoust Soc Am 72: 1441-9.

Liberman MC. 1990. Effects of chronic cochlear de-efferentation on auditory-nerve response. Hear Res 49: 209-23.

Liberman MC, Dodds LW, Pierce S. 1990. Afferent and efferent innervation of the cat cochlea: quantitative analysis with light and electron microscopy. J Comp Neurol 301: 44360 .

Lorincz A, Nusser Z. 2008. Cell-type-dependent molecular composition of the axon initial segment. J Neurosci 28: 14329-40.

Lumpkin EA, Hudspeth AJ. 1995. Detection of Ca2+ entry through mechanosensitive channels localizes the site of mechanoelectrical transduction in hair cells. Proc Natl Acad Sci U S A 92: 10297-301.

Lumpkin EA, Marquis RE, Hudspeth AJ. 1997. The selectivity of the hair cell's mechanoelectrical-transduction channel promotes $\mathrm{Ca} 2+$ flux at low $\mathrm{Ca} 2+$ concentrations. Proc Natl Acad Sci U S A 94: 10997-1002.

Maison SF, Adams JC, Liberman MC. 2003. Olivocochlear innervation in the mouse: immunocytochemical maps, crossed versus uncrossed contributions, and transmitter colocalization. J Comp Neurol 455: 406-416.

Marcotti W, Johnson SL, Rusch A, Kros CJ. 2003. Sodium and calcium currents shape action potentials in immature mouse inner hair cells. $J$ Physiol 552: 743-61.

Martinez-Dunst C, Michaels RL, Fuchs PA. 1997. Release sites and calcium channels in hair cells of the chick's cochlea. J Neurosci 17: 9133-44.

Martini M, Rossi ML, Rubbini G, Rispoli G. 2000. Calcium currents in hair cells isolated from semicircular canals of the frog. Biophys $J$ 78: 1240-54.

Matthews G, Sterling P. 2008. Evidence that vesicles undergo compound fusion on the synaptic ribbon. $J$ Neurosci 28: 5403-11.

Matveev V, Bertram R, Sherman A. 2009. Ca2+ current versus $\mathrm{Ca} 2+$ channel cooperativity of exocytosis. J Neurosci 29: 12196-209.

Matveev V, Sherman A, Zucker RS. 2002. New and corrected simulations of synaptic facilitation. Biophys $J$ 83: 1368-73.

Meddis R. 1986. Simulation of mechanical to neural transduction in the auditory receptor. $J$ Acoust Soc Am 79: 702-11.

Meddis R. 1990. Implementation details of a computation model of the inner hair-cell auditory-nerve synapse. J Acoust Soc Am 87: 1813. 
Merchan-Perez A, Liberman MC. 1996. Ultrastructural differences among afferent synapses on cochlear hair cells: correlations with spontaneous discharge rate. J Comp Neurol 371: 208-21.

Meyers JR, MacDonald RB, Duggan A, Standaert DG, Lenzi D, Corwin JT, Corey DP. 2003. Lighting up the senses: FM1-43 loading of sensory cells through nonselective ion channels. J Neurosci 23: 4054-65.

Miesenböck G, De Angelis DA, Rothman JE. 1998. Visualizing secretion and synaptic transmission with $\mathrm{pH}$-sensitive green fluorescent proteins. Nature 394: 192-5.

Mo ZL, Davis RL. 1997. Heterogeneous voltage dependence of inward rectifier currents in spiral ganglion neurons. J Neurophysiol 78: 3019-27.

Moser T, Neef A, Khimich D. 2006. Mechanisms underlying the temporal precision of sound coding at the inner hair cell ribbon synapse. J Physiol 576: 55-62.

Moller AR. 2006. Hearing: Anatomy, Physiolgy, and Disorders of the Auditory System. Academic Press.

Müller U. 2008. Cadherins and mechanotransduction by hair cells. Current opinion in cell biology 20: 557-66.

Nadol JB. 1983. Serial section reconstruction of the neural poles of hair cells in the human organ of Corti.I. Inner hair cells. Laryngoscope 93: 599-614.

Nadol JB. 1988. Comparative anatomy of the cochlea and auditory nerve in mammals. Hear Res 34: 253-66.

Naraghi M. 1997. T-jump study of calcium binding kinetics of calcium chelators. Cell calcium 22: 255-68.

Neef J, Gehrt A, Bulankina AV, Meyer AC, Riedel D, Gregg RG, Strenzke N, Moser T. 2009. The $\mathrm{Ca} 2+$ channel subunit beta 2 regulates $\mathrm{Ca} 2+$ channel abundance and function in inner hair cells and is required for hearing. $J$ Neurosci 29: 10730-40.

Neef A, Khimich D, Pirih P, Riedel D, Wolf F, Moser T. 2007. Probing the mechanism of exocytosis at the hair cell ribbon synapse. J Neurosci 27: 12933-44.

Neher E. 1998. Vesicle pools and $\mathrm{Ca} 2+$ microdomains: new tools for understanding their roles in neurotransmitter release. Neuron 20: 389-99.

Neher E, Augustine GJ. 1992. Calcium gradients and buffers in bovine chromaffin cells. $J$ Physiol 450: 273-301.

Neher E, Sakaba T. 2008. Multiple roles of calcium ions in the regulation of neurotransmitter release. Neuron 59: 861-72. 
Nin F, Hibino H, Doi K, Suzuki T, Hisa Y, Kurachi Y. 2008. The endocochlear potential depends on two $\mathrm{K}+$ diffusion potentials and an electrical barrier in the stria vascularis of the inner ear. Proc Natl Acad Sci U S A 105: 1751-6.

Nägerl UV, Novo D, Mody I, Vergara JL. 2000. Binding kinetics of calbindin-D(28k) determined by flash photolysis of caged $\mathrm{Ca}(2+)$. Biophys $J$ 79: 3009-18.

Oliver D, Klöcker N, Schuck J, Baukrowitz T, Ruppersberg JP, Fakler B. 2000. Gating of $\mathrm{Ca} 2+$-activated $\mathrm{K}+$ channels controls fast inhibitory synaptic transmission at auditory outer hair cells. Neuron 26: 595-601.

Oliver D, Knipper M, Derst C, Fakler B. 2003. Resting potential and submembrane calcium concentration of inner hair cells in the isolated mouse cochlea are set by KCNQ-type potassium channels. $J$ Neurosci 23: 2141-9.

Palmer AR, Russell IJ. 1986. Phase-locking in the cochlear nerve of the guinea-pig and its relation to the receptor potential of inner hair-cells. Hear Res 24: 1-15.

Platzer J, Engel J, Schrott-Fischer A, Stephan K, Bova S, Chen H, Zheng H, Striessnig J. 2000. Congenital deafness and sinoatrial node dysfunction in mice lacking class D L-type Ca2+ channels. Cell 102: 89-97.

Purves D, Augustine GJ, Chikaraishi DM, Ehlers MD, Einstein G, Fitzpatrick D, Hall WC, Jarvis E, Katz LC, Kauer J, LaMantia A, McNamara JO, Mooney RD, Nicolelis MA, Reinhart PH, Simon SA, Skene JH, Voyvodic J, White LE, Williams MS. 2004. Neuroscience (D Purves, GJ Augustine, D Fitzpatrick, WC Hall, A LaMantia, JO McNamara, and MS Williams). Sinauer Associates.

Reichenbach T, Hudspeth AJ. 2010. A ratchet mechanism for amplification in lowfrequency mammalian hearing. Proc Natl Acad Sci U S A Epub ahead.

Ricci A. 2002. Differences in mechano-transducer channel kinetics underlie tonotopic distribution of fast adaptation in auditory hair cells. Journal of neurophysiology 87: 1738-48.

Ricci AJ, Gray-Keller M, Fettiplace R. 2000. Tonotopic variations of calcium signalling in turtle auditory hair cells. J Physiol 524 Pt 2: 423-36.

Rispoli G, Martini M, Rossi ML, Mammano F. 2001. Dynamics of intracellular calcium in hair cells isolated from the semicircular canal of the frog. Cell calcium 30: 131-40.

Roberts WM. 1993. Spatial calcium buffering in saccular hair cells. Nature 363: 74-6.

Roberts WM. 1994. Localization of calcium signals by a mobile calcium buffer in frog saccular hair cells. $J$ Neurosci 14: 3246-62.

Roberts WM, Jacobs RA, Hudspeth AJ. 1990. Colocalization of ion channels involved in frequency selectivity and synaptic transmission at presynaptic active zones of hair cells. $J$ Neurosci 10: 3664-84. 
Robertson D, Paki B. 2002. Role of L-type Ca2+ channels in transmitter release from mammalian inner hair cells. II. Single-neuron activity. J Neurophysiol 87: 2734-40.

Robles L, Ruggero MA. 2001. Mechanics of the mammalian cochlea. Physiol Rev 81: 130552 .

Rodriguez-Contreras A, Yamoah EN. 2001. Direct measurement of single-channel $\mathrm{Ca}(2+)$ currents in bullfrog hair cells reveals two distinct channel subtypes. $J$ Physiol 534: 669-89.

Roux I, Safieddine S, Nouvian R, Grati M, Simmler MC, Bahloul A, Perfettini I, Le Gall M, Rostaing P, Hamard G, Triller A, Avan P, Moser T, Petit C. 2006. Otoferlin, defective in a human deafness form, is essential for exocytosis at the auditory ribbon synapse. Cell 127: 277-289.

Ruel J, Bobbin RP, Vidal D, Pujol R, Puel JL. 2000. The selective AMPA receptor antagonist GYKI 53784 blocks action potential generation and excitotoxicity in the guinea pig cochlea. Neuropharmacology 39: 1959-73.

Ruel J, Emery S, Nouvian R, Bersot T, Amilhon B, Van Rybroek JM, Rebillard G, Lenoir M, Eybalin M, Delprat B, Sivakumaran TA, Giros B, El Mestikawy S, Moser T, Smith RJ, Lesperance MM, Puel J. 2008. Impairment of SLC17A8 encoding vesicular glutamate transporter-3, VGLUT3, underlies nonsyndromic deafness DFNA25 and inner hair cell dysfunction in null mice. Am J Hum Genet 83: 278-92.

Ruel J, Nouvian R, Chabbert C, Bendris R, Eybalin M, Leger CL, Bourien J, Mersel M, Puel J. 2008. Salicylate enables cochlear arachidonic-acid-sensitive NMDA receptor responses. J Neurosci 28: 7313-23.

Ruel J, Nouvian R, Gervais D'Aldin C, Pujol R, Eybalin M, Puel JL. 2001. Dopamine inhibition of auditory nerve activity in the adult mammalian cochlea. Eur J Neurosci 14: 97786.

Russell IJ, Sellick PM. 1978. Intracellular studies of hair cells in the mammalian cochlea. $J$ Physiol 284: 261-90.

Russell IJ, Sellick PM. 1983. Low-frequency characteristics of intracellularly recorded receptor potentials in guinea-pig cochlear hair cells. J Physiol 338: 179-206.

Rutherford MA, Roberts WM. 2006. Frequency selectivity of synaptic exocytosis in frog saccular hair cells. Proc Natl Acad Sci U S A 103: 2898-2903.

Rutherford MA, Roberts WM. 2009. Spikes and membrane potential oscillations in hair cells generate periodic afferent activity in the frog sacculus. J Neurosci 29: 10025-37.

Safieddine S, Wenthold RJ. 1999. SNARE complex at the ribbon synapses of cochlear hair cells: analysis of synaptic vesicle- and synaptic membrane-associated proteins. Eur J Neurosci 11: 803-12.

Schmitz F. 2009. The Making of Synaptic Ribbons: How They Are Built and What They Do. Neuroscientist 15: 611-24. 
Schnee ME, Ricci AJ. 2003. Biophysical and pharmacological characterization of voltagegated calcium currents in turtle auditory hair cells. J Physiol 549: 697-717.

Schnee ME, Furness DN, Lawton DM, Benke TA, Ricci AJ. 2005. Auditory hair cellafferent fiber synapses are specialized to operate at their best frequencies. Neuron 47: 243-54.

Seal RP, Akil O, Yi E, Weber CM, Grant L, Yoo J, Clause A, Kandler K, Noebels JL, Glowatzki E, Lustig LR, Edwards RH. 2008. Sensorineural deafness and seizures in mice lacking vesicular glutamate transporter 3. Neuron 57: 263-75.

Shuai J, Parker I. 2005. Optical single-channel recording by imaging Ca2+ flux through individual ion channels: theoretical considerations and limits to resolution. Cell calcium 37: 283-99.

Siegel JH. 1992. Spontaneous synaptic potentials from afferent terminals in the guinea pig cochlea. Hear Res 59: 85-92.

Silver RA, Lamb AG, Bolsover SR. 1990. Calcium hotspots caused by L-channel clustering promote morphological changes in neuronal growth cones. Nature 343: 751-4.

Singer JH, Glowatzki E, Moser T, Strowbridge BW, Bhandawat V, Sampath AP. 2009. Functional properties of synaptic transmission in primary sense organs. J Neurosci 29: 128026.

Singer JH, Lassová L, Vardi N, Diamond JS. 2004. Coordinated multivesicular release at a mammalian ribbon synapse. Nat Neurosci 7: 826-33.

Singh A, Gebhart M, Fritsch R, Sinnegger-Brauns MJ, Poggiani C, Hoda J, Engel J, Romanin C, Striessnig J, Koschak A. 2008. Modulation of voltage- and Ca2+-dependent gating of CaV1.3 L-type calcium channels by alternative splicing of a C-terminal regulatory domain. J Biol Chem 283: 20733-44.

Singh A, Hamedinger D, Hoda J, Gebhart M, Koschak A, Romanin C, Striessnig J. 2006. C-terminal modulator controls $\mathrm{Ca} 2+$-dependent gating of $\mathrm{Ca}(\mathrm{v}) 1.4 \mathrm{~L}$-type $\mathrm{Ca} 2+$ channels. Nat Neurosci 9: 1108-16.

Slepecky NB. 1996. Structure of the mammalian cochlea. In: Dallos, P, Popper, AN, Fay, RR, The Cochlea. New York: Springer, 44-129.

Stanley EF. 1997. The calcium channel and the organization of the presynaptic transmitter release face. Trends Neurosci 20: 404-9.

Strenzke N, Chanda S, Kopp-Scheinpflug C, Khimich D, Reim K, Bulankina AV, Neef A, Wolf F, Brose N, Xu-Friedman MA, Moser T. 2009. Complexin-I is required for highfidelity transmission at the endbulb of held auditory synapse. J Neurosci 29: 7991-8004.

Sumner CJ, Lopez-Poveda EA, O'Mard LP, Meddis R. 2002. A revised model of the inner-hair cell and auditory-nerve complex. J Acoust Soc Am 111: 2178-88. 
Sumner CJ, OMard LP, Lopez-Poveda EA, Meddis R. 2003. A nonlinear filter-bank model of the guinea-pig cochlear nerve: Rate responses. J Acoust Soc Am 113: 3264.

Südhof TC. 2004. The synaptic vesicle cycle. Annu Rev Neurosci 27: 509-47.

Taberner AM, Liberman MC. 2005. Response properties of single auditory nerve fibers in the mouse. $J$ Neurophysiol 93: 557-69.

Takahashi A, Camacho P, Lechleiter JD, Herman B. 1999. Measurement of intracellular calcium. Physiol Rev 79: 1089-125.

Thurm H, Fakler B, Oliver D. 2005. Ca2+-independent activation of BKCa channels at negative potentials in mammalian inner hair cells. $J$ Physiol 569: 137-51.

Tritsch NX, Bergles DE. 2010. Developmental regulation of spontaneous activity in the Mammalian cochlea. $J$ Neurosci 30: 1539-50.

Tritsch NX, Yi E, Gale JE, Glowatzki E, Bergles DE. 2007. The origin of spontaneous activity in the developing auditory system. Nature 450: 50-5.

Trussell LO. 2002. Transmission at the hair cell synapse. Nat Neurosci 5: 85-6.

Tucker T, Fettiplace R. 1995. Confocal imaging of calcium microdomains and calcium extrusion in turtle hair cells. Neuron 15: 1323-35.

Viemeister NF. 1988. Intensity coding and the dynamic range problem. Hear Res 34: 267-74.

Wangemann P, Schacht J. 1996. Homeostatic Mechanisms in the Cochlea. In: Dallos, P, Popper, AN, Fay, RR, The Cochlea. New York: Springer, 130-185.

Weisz C, Glowatzki E, Fuchs P. 2009. The postsynaptic function of type II cochlear afferents. Nature 461: 1126-9.

Wen B, Wang GI, Dean I, Delgutte B. 2009. Dynamic range adaptation to sound level statistics in the auditory nerve. $J$ Neurosci 29: 13797-808.

Yamada WM, Zucker RS. 1992. Time course of transmitter release calculated from simulations of a calcium diffusion model. Biophys $J$ 61: 671-82.

Zampini V, Johnson SL, Franz C, Lawrence ND, Münkner S, Engel J, Knipper M, Magistretti J, Masetto S, Marcotti W. 2010. Elementary properties of $\mathrm{CaV} 1.3 \mathrm{Ca}(2+)$ channels expressed in mouse cochlear inner hair cells. J Physiol 588: 187-99.

Zenisek D, Matthews G. 2000. The role of mitochondria in presynaptic calcium handling at a ribbon synapse. Neuron 25: 229-37.

Zenisek D, Davila V, Wan L, Almers W. 2003. Imaging calcium entry sites and ribbon structures in two presynaptic cells. $J$ Neurosci 23: 2538-48. 
Zenisek D, Horst NK, Merrifield C, Sterling P, Matthews G. 2004. Visualizing synaptic ribbons in the living cell. $J$ Neurosci 24: 9752-9. 


\section{Acknowledgements}

First, I thank Prof. Tobias Moser for giving me the opportunity to work in his laboratory, especially on this particular project. I acknowledge his constant support and never ending interest, his guidance, the scientific discussions and his continuous efforts to foster exchange and connections with colleagues from within and outside the field.

I also thank Prof. Detlev Schild and Prof. Erwin Neher, the additional members of my thesis committee, for their time, critical feedback, and useful advices.

I am very grateful to all the members of the InnerEarLab, first and foremost Dr. Andreas Neef and Dr. Regís Nouvian, for many helpful discussions and advice but also for constructing a very enjoyable working environment. Many thanks also to Margitta Köppler, Sonja Blume, and Nina Dankenbrink-Werder for their excellent technical assistance.

Furthermore, I thank Prof. David Zenisek and Prof. Gary Matthews for providing us with their fluorescently labeled ribeye-binding peptides.

I also thank Prof. Michael Hörner and Sandra Drube from the Neurosciences coordination office for their uncomplicated, quick, and ongoing help and support in organizational matters.

I furthermore acknowledge the financial support provided by the state of Lower Saxony in the form of a Georg-Christoph-Lichtenberg stipend, and by the Novartis Foundation of Therapeutic Research.

Finally, I like to thank my family and friends for their unfailing support, understanding, and encouragement. 


\section{Curriculum Vitae}

Full name: Thomas Frank

Date of birth: February 04, 1982

Place of birth: Starnberg, Germany

Citizenship: German

\section{Education}

2001

Abitur

Graf-Rasso-Gymnasium Fürstenfeldbruck, Germany

$2002-2004$

'Vordiplom' in biology

University of Giessen, Germany

$2004-2005$

Two semesters 'Hauptstudium' in neurobiology

University of Magdeburg, Germany

$2005-2006$

MSc study year

International Max-Planck Research School for Neurosciences, Göttingen, Germany

$2006-2007$

\section{MSc in neurosciences}

MSc thesis with Prof. Tobias Moser at the InnerEarLab, Department of Otorhinolaryngology, University of Göttingen

2007 - present $\quad$ PhD student

PhD thesis with Prof. Tobias Moser at the InnerEarLab, Department of Otorhinolaryngology, University of Göttingen 


\section{Publication list}

2010

Pangršič, T., Lasarow, L., Reuter, K., Takago, H., Schwander, M., Riedel, D., Frank, T., Tarantino, L. M., Bailey, J. S., Strenzke, N., Brose, N., Müller, U., Reisinger, E., Moser, T. (2010). Hearing requires otoferlin-dependent efficient replenishment of synaptic vesicles in hair cells (under review).

Meyer, A. C.*, Frank, T.*, Khimich, D.*, Hoch, G., Riedel, D., Chapochnikov, N. M., Yarin, Y. M., Harke, B., Hell, S. W., Egner, A., $\&$ Moser, T. (2009). Tuning of synapse number, structure and function in the cochlea. Nat Neurosci, 12 (4), 444-453.

(* these authors contributed equally to the work)

Frank, T., Khimich, D., Neef, A., \& Moser, T. (2009). Mechanisms contributing to synaptic $\mathrm{Ca}^{2+}$ signals and their heterogeneity in hair cells. Proc Natl Acad Sci US A, 106 (11), 4483-4488. 